\title{
Synchronization of Mechanical Systems
}




\title{
CIP-DATA LIBRARY TECHNISCHE UNIVERSITEIT EINDHOVEN
}

\author{
Rodriguez Angeles, Alejandro
}

Synchronization of Mechanical Systems / by Alejandro Rodriguez Angeles. Eindhoven: Technische Universiteit Eindhoven, 2002.

Proefschrift. - ISBN 90-386-2634-7

NUR 950

Subject headings: control systems technology / mechanical systems; robotics / synchronization / coupled dynamical systems / system stability; Lyapunov methods / feedback control / observers

Trefwoorden: regeltechnische systemen / mechanische systemen; robotica / synchronisatie / gekoppelde dynamische systemen / stabiliteit; Lyapunov methoden / feedback control / observers

Printed by University Press Facilities, Eindhoven, The Netherlands

Cover design by Paul Verspaget

Copyright (c) 2002, by Alejandro Rodriguez Angeles

This dissertation has been completed in partial fulfillment of the requirements of the Dutch Institute of Systems and Control (DISC) for graduate study.

This project has been partially supported by the Eindhoven University of Technology (Technische Universiteit Eindhoven) and by a scholarship granted by The National Council for Science and Technology (CONACyT), México. 


\section{Synchronization of Mechanical Systems}

\section{PROEFSCHRIFT}

ter verkrijging van de graad van doctor aan de

Technische Universiteit Eindhoven,

op gezag van de Rector Magnificus, prof.dr. R.A. van Santen, voor een commissie aangewezen door het College voor Promoties

in het openbaar te verdedigen op

woensdag 30 oktober 2002 om 16.00 uur

door

\section{Alejandro Rodriguez Angeles}

geboren te Tepeji del Rio, Mexico 
Dit proefschrift is goedgekeurd door de promotoren:

prof.dr. H. Nijmeijer

en

prof.dr.ir. J.J. Kok

Copromotor:

dr.ir. H.A. van Essen 


\section{Preface}

It has been almost four years since I decided to come to The Netherlands to become a $\mathrm{PhD}$ student. The document you have in your hands should give an overview of what I have been doing in the scientific part of those four years. I mean in the scientific part because it has resulted in a larger enterprize than what I thought, and with more things involved than only science.

Although this thesis is related to the scientific part of my $\mathrm{PhD}$, the preface is more oriented to the personal part running parallel (should I say synchronized ?) to the $\mathrm{PhD}$ project. First of all being abroad from my motherland has made me see things from a different perspective, not only scientific but also social. I expect this new perspective will help me to do something better for myself, my family and the society in my motherland. Second, but quite important as well, is that being abroad has given me the opportunity to meet different cultures, ways of thinking, and overall many people. Indeed, I am indebted to many people who have contributed directly or indirectly to the realization of this thesis.

A special word or thanks goes to my supervisor and promotor prof.dr. Henk Nijmeijer. His enthusiasm, confidence and encouraging words helped me a lot to successfully finish my research. It has been a great pleasure to work with him. I hope the same goes for him, although he must have gotten fed up of my "Spanglish" and the reading of several drafts of this thesis.

The last line applies also to my second promotor prof.dr.ir. Jan Kok and copromotor dr.ir. Harm van Essen. Thanks to both of you for helping me to write down my ideas. This thesis would not read the way it does without their many remarks and suggestions. Special thanks to Harm, who translated the "samenvatting" (summary in Dutch) which can be found at the end of the thesis.

I am grateful to all the members of my promotion committee for thorough reading the manuscript: prof.dr. H. Nijmeijer, prof.dr.ir. J.J. Kok, prof.dr.ir. M.H.G. Verhaegen, prof.dr.ir. H.J.C. Huijberts, prof.dr.ir. H. Kwakernaak, prof.dr.ir. P.P.J. van den Bosch, prof.dr.ir. M. Steinbuch and dr.ir. H.A. van Essen.

I would like to thank to my former colleagues at the University of Twente, especially for providing me with such a friendly working atmosphere. Maria, thanks a lot for your friendship and for the many teas we enjoyed at the office. Goran, it has been a pleasure to work with such a creative and capable person. Special thanks go to Marja Langkamp for helping me a lot with my coming to The Netherlands and the adaptation period during the first months.

Also many thanks to my colleagues and friends at Eindhoven, Edo, Dragan, Wilbert, Nenad, David, Alejandra, Sasha and many others whose names would make this list endless. I will never forget the nice and creative hours and discussions we had in front 
of Lia's office. Lia thanks for your help in the administrative matters that I had to go trough during my stay in Eindhoven.

This thesis would not have been finished without the assistance of the people at the Dynamics and Control Laboratory: Niels Olthuis, Rens Kodde, Harrie van de Loo and Karel Koekkoek. Karel, thanks for setting up the robots for the experiments and being so promptly to help me in the technical problems related with the synchronization setup.

I am also indebted to all the people who make my life in The Netherlands enjoyable and happy. Cami and Hans thanks for your friendship and the nice talks we have during the dinners and barbecues that I have become so fond of.

Thanks to my friends and former colleagues from Mexico for their support and encouraging in pursuing the $\mathrm{PhD}$.

Last but not least, many thanks to Magda and my family for their support and help through this enterprize that has been the PhD. Pupica thanks for showing me the human side of life. I like to mention specially my mother, who suffered a lot from my absence. For this reason this work is entirely dedicated to her.

Gracias Mama por tu apoyo y cariño. Esta tesis es por tí.

Alejandro Rodriguez Angeles. 


\section{Contents}

Preface $v$

1 Introduction $\quad \mathbf{5}$

1.1 General introduction . . . . . . . . . . . . . . . . . 5

1.2 Synchronization . . . . . . . . . . . . . . . . 7

1.3 Synchronization in robotic systems $\ldots \ldots \ldots \ldots \ldots \ldots$

1.3.1 Velocity and acceleration measurements . . . . . . . . 9

$1.3 .2 \quad$ Joint flexibility . . . . . . . . . . . . . . . . . . . . . 9

1.3.3 Friction Phenomena . . . . . . . . . . . . . . . . . 9

1.4 Problem formulation . . . . . . . . . . . . . . . . . . 10

1.4.1 External synchronization of rigid joint robots . . . . . . . 10

1.4.2 External synchronization of flexible joint robots . . . . . . . . 11

1.4.3 Mutual (internal) synchronization of rigid joint robots . . . . 12

1.5 Goals and main contributions of the thesis . . . . . . . . . . . 12

1.6 Outline of the thesis . . . . . . . . . . . . . . . . 14

2 Preliminaries $\quad 15$

2.1 Mathematical preliminaries and stability concepts . . . . . . . . 15

2.1 .1 Basic definitions . . . . . . . . . . . . . . . 15

2.1 .2 Lyapunov Stability . . . . . . . . . . . . . . . . . . . 17

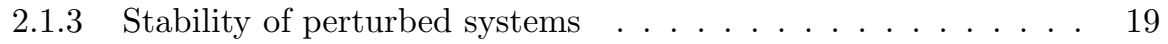

2.2 Dynamic models of robot manipulators . . . . . . . . . . . . . . 21

2.2 .1 Rigid joint robots . . . . . . . . . . . . . . . . . . 21

$2.2 .2 \quad$ Flexible joint robots . . . . . . . . . . . . . . . 21

2.2.3 Properties of the dynamic model of the robots . . . . . . . 23

2.2.4 Friction phenomena . . . . . . . . . . . . . . . 24

2.3 Experimental setup . . . . . . . . . . . . . . . . . 26

3 External synchronization of rigid joint robots 29

3.1 Introduction . . . . . . . . . . . . . . . . . 29

3.2 Synchronization controller based on state feedback . . . . . . . . . 31

3.3 Synchronization controller based on estimated variables . . . . . . . 32

3.3.1 Feedback control law . . . . . . . . . . . . . . . . 32

3.3.2 An observer for the synchronization errors $e, \dot{e} \ldots \ldots . . .32$

3.3.3 An observer for the slave joint variables $q_{s}, \dot{q}_{s} \ldots \ldots \ldots \ldots$

3.3.4 Estimated values for $\dot{q}_{m}, \ddot{q}_{m} \ldots \ldots \ldots \ldots \ldots$

3.3.5 Synchronization closed loop error dynamics . . . . . . . . . 33 
3.3.6 Stability analysis . . . . . . . . . . . . . . . . . 35

3.4 Gain tuning procedure . . . . . . . . . . . . . . . . . . . 40

3.5 Friction compensation . . . . . . . . . . . . . . . . . . 41

3.6 Simulation and experimental study . . . . . . . . . . . . . . . 42

3.7 Concluding remarks and discussion $\ldots \ldots \ldots \ldots . \ldots . \ldots 4$

4 External synchronization of flexible joint robots 49

4.1 Introduction . . . . . . . . . . . . . . . . . . . . 49

4.2 Synchronization controller based on state feedback . . . . . . . . . 51

4.3 Synchronization controller based on estimated variables . . . . . . . 52

4.3.1 An observer for the synchronization errors $e, \dot{e} \ldots \ldots \ldots$

4.3.2 An observer for the slave variables $q_{s}, \dot{q}_{s}, \theta_{s}, \dot{\theta}_{s} \ldots \ldots \ldots 53$

4.3.3 Synchronization closed loop error dynamics . . . . . . . . . 54

4.3 .4 Stability analysis . . . . . . . . . . . . . . 55

4.4 Gain tuning procedure . . . . . . . . . . . . . . . 61

4.5 Simulation study . . . . . . . . . . . . . . . . . . . . . 62

4.6 Concluding remarks and discussion . . . . . . . . . . . 64

5 Mutual synchronization of rigid joint robots 65

5.1 Introduction . . . . . . . . . . . . . . . . 65

5.2 Synchronization controller based on state feedback . . . . . . . . 67

5.2 .1 Synchronization closed loop error dynamics . . . . . . . . 68

5.2 .2 Stability analysis . . . . . . . . . . . . . . . 68

5.2 .3 Algebraic loop . . . . . . . . . . . . . . . . . 72

5.3 Synchronization controller based on estimated variables . . . . . . . 72

5.3 .1 An observer for the joint variables . . . . . . . . . . 73

5.3 .2 Synchronization closed loop error dynamics . . . . . . . . 75

5.3 .3 Stability analysis . . . . . . . . . . . . . . 76

5.4 Gain tuning procedure . . . . . . . . . . . . . . . . 80

5.5 Friction compensation . . . . . . . . . . . . . . . 81

5.6 Simulation and experimental study . . . . . . . . . . . . . . . 81

5.7 Concluding remarks and discussion . . . . . . . . . . . . 87

6 Experimental case study $\quad 89$

6.1 Introduction . . . . . . . . . . . . . . . . . . . 89

6.2 The CFT transposer robot . . . . . . . . . . . . . . . . 89

6.2 .1 Joint space dynamics . . . . . . . . . . . . . . . 90

6.3 External synchronization of a complex multi-robot system . . . . . . 91

6.4 Mutual synchronization of a complex multi-robot system . . . . . . 94

6.5 Conclusions and discussion . . . . . . . . . . . . . . . 98

$\begin{array}{lll}7 & \text { Extensions } & 99\end{array}$

7.1 Leader-follower synchronization of mobile robots . . . . . . . . . . 99

7.1.1 Kinematic model of the mobile robot . . . . . . . . . 100

7.1.2 Leader-follower synchronization controller . . . . . . . . . . 100

7.1 .3 Simulation study . . . . . . . . . . . . . . . . 101

7.2 Attitude formations of multi-satellite systems . . . . . . . . . . 103

7.2.1 Dynamics of the satellite system . . . . . . . . . . . . 103

7.2.2 Synchronization strategy and controller . . . . . . . . 105 
7.2 .3 Simulation study . . . . . . . . . . . . . . . . . 106

7.3 Discussion . . . . . . . . . . . . . . . . . . . . 108

8 Conclusions and recommendations 109

8.1 Concluding remarks . . . . . . . . . . . . . . . . . . . . . . 109

8.2 Recommendations . . . . . . . . . . . . . . . . . . . 111

$\begin{array}{lr}\text { A Proof of Lemma 3.9 } & 113\end{array}$

$\begin{array}{ll}\text { B Proof of Theorem } 3.12 & 117\end{array}$

$\begin{array}{lr}\text { C Proof of Lemma 4.1 } & 121\end{array}$

$\begin{array}{ll}\text { D Proof of Lemma } 4.7 & 123\end{array}$

$\begin{array}{ll}\text { E Proof of Proposition 4.9 } & 127\end{array}$

$\begin{array}{lll}\text { F } & \text { Proof of Lemma 5.9 } & 129\end{array}$

$\begin{array}{ll}\text { G Proof of Theorem 5.11 } & 131\end{array}$

H Dynamic model of the CFT robot 133

$\begin{array}{ll}\text { Bibliography } & 147\end{array}$

$\begin{array}{ll}\text { Summary } & 153\end{array}$

$\begin{array}{lr}\text { Samenvatting } & 155\end{array}$

$\begin{array}{ll}\text { Resumen } & 157\end{array}$

$\begin{array}{lr}\text { Curriculum vitae } & 159\end{array}$ 


\section{Chapter 1}

\section{Introduction}

\subsection{General introduction}

Nowadays the developments in technology and the requirements on efficiency and quality in production processes have resulted in complex and integrated production systems. In actual production processes such as manufacturing, automotive applications, and teleoperation systems there is a high requirement on flexibility and manoeuvrability of the involved systems. In most of these processes the use of integrated and multi-composed systems is widely spread, and their variety in uses is practically endless; assembling, transporting, painting, welding, just to mention few. All these tasks require large manoeuvrability and manipulability of the executing systems, often even some of the tasks can not be carried out by a single system. In those cases the use of multi-composed systems has been considered as an option. A multi-composed system is a group of individual systems, either identical or different, that work together to execute a task.

In practice many multi-composed systems work either under cooperative or under coordinated schemes. In cooperative schemes there are interconnections between all the systems, such that all systems have influence on the combined dynamics, while in coordinated schemes there are only interconnections from the leader or dominant system to the non-dominant ones. Therefore in coordinated schemes the leader system determines the synchronized behavior of all the non-dominant systems. Note that coordinated and cooperative systems are nothing else that a requirement of synchronous behavior of the multi-composed system. Synchronization, coordination, and cooperation are intimately linked subjects and very often, mainly in mechanical systems, they are used as synonymous to describe the same kind of behavior.

The synchronization phenomenon was perhaps first reported by Huygens (1673), who observed that a pair of pendulum clocks hanging from a light weight beam oscillated with the same frequency. Synchronized sound in nearby organ tubes was reported by Rayleigh in 1877 (Rayleigh 1945), who observed similar effects for two electrically or mechanically connected tuning forks. In the last century synchronization received a lot of attention in the Russian scientific community since it was observed in balanced and unbalanced rotors and vibro-exciters (Blekhman 1988). Nowadays, there are several papers related with synchronization of rotating bodies and electromechanical systems (Blekhman et al. 1995), (Huijberts et al. 2000). On the one hand, rotating 
mechanical structures form a very important and special class of systems that, with or without the interaction through some coupling, exhibit synchronized motion, for example the case of vibro-machinery in production plants, electrical generators, unbalanced rotors in milling machines (Blekhman 1988). On the other hand, for mechanical systems synchronization is of great importance as soon as two machines have to cooperate. The cooperative behavior gives flexibility and manoeuvrability that cannot be achieved by an individual system, e.g. multi finger robot-hands, multi robot systems and multi-actuated platforms (Brunt 1998), (Liu et al. 1999), teleoperated masterslave systems (Dubey et al. 1997), (Lee and Chung 1998). In medicine master-slave teleoperated systems are used in surgery giving rise to more precise and less invasive surgery procedures (Hills and Jensen 1998), (Guthart and Salisbury 2000). In aerospace applications coordination schemes are used to minimize the error of the relative attitude in formations of satellites (Wang et al. 1996), (Kang and Yeh 2002). The case of group formation of multiple robotic vehicles is addressed in (Yamaguchi et al. 2001).

Nevertheless mechanical systems are not the only application in which synchronization plays an important role. In communication systems synchronization is used to improve the efficiency of the transmitter-receiver systems, also synchronization and chaos have been used to encrypt information improving security in the transmissions (Pecora and Carroll 1990), (Cuomo et al. 1993), (Kocarev et al. 1992).

The importance of synchronization does not only lie in the practical applications that can be obtained, but also in the many phenomena that can be explained by synchronization theory. In astronomy synchronization theory is used to explain the motion of celestial bodies, such as orbits and planetary resonances, (Blekhman 1988). In biology, biochemistry and medicine many systems can be modelled as oscillatory or vibratory systems and those systems show a tendency towards synchronous behavior. Synchronous activity has been observed in many regions of the human brain, relative to behavior and cognition, where neurons can synchronously discharge in some frequency ranges (Gray 1994). Synchronous firing of cardiac pacemaker cells in human heart has been reported in (Torre 1976). Meanwhile evidence of synchronicity among pulse-coupled biological oscillators has been presented in (Mirollo and Strogatz 1990). Among evidences of synchronous behavior in the natural world, one can consider the chorusing of crickets, synchronous flash light in group of fire-flies, and the metabolic synchronicity in yeast cell suspension, see (Winfree 1980).

Notice that synchronization in the above mentioned physical phenomena, such as biology and astronomy, appears in a natural way and is due only to the proper couplings of the systems, which is called self-synchronization. This is the main difference with respect to the practical applications of synchronization theory, where the synchronous behavior is induced by means of artificial couplings and inputs, such as feedback and feedforward controllers. This is the so-called controlled synchronization.

This thesis focuses on controlled synchronization of robot systems, which nowadays are common and important systems in production processes. However, the general ideas developed here can be extended to more general mechanical systems, such as mobile robots, motors, balanced and unbalanced rotors, vibro-exciters. For better understanding of the controlled synchronization problem first a general definition of synchronization is introduced, second the particular problems in the case of controlled robot synchronization are briefly listed. 


\subsection{Synchronization}

According to (Blekhman et al. 1995) synchronization may be defined as the mutual time conformity of two or more processes. This conformity can be characterized by the appearance of certain relations between some functionals for the processes. Furthermore, based on the type of interconnections (interactions) in the system, different kinds of synchronization can be defined (Blekhman et al. 1997).

- In case of disconnected systems that present synchronous behavior this is referred to as natural synchronization, e.g. all precise clocks are synchronized in the frequency domain.

- When synchronization is achieved by proper interconnections in the systems, i.e. without any artificially introduced external action, then the system is referred to as self-synchronized. A classical example of self-synchronization is the pair of pendulum clocks hanging from a light weight beam that was reported by Huygens (1673). He observed that both pendulums oscillated with the same frequency. Another example is the synchronization of celestial bodies, such as rotation of satellites around planets.

- When there exist external actions (input controls) and/or artificial interconnections then the system is called controlled-synchronized, examples of this case are most of the practical applications of synchronization theory such as transmitterreceiver systems and synchronized oscillators in communications.

Depending on the formulation of the controlled synchronization problem distinction should be made between internal (mutual) synchronization and external synchronization.

- In the first and most general case, all synchronized objects occur on equal terms in the unified multi-composed system. Therefore the synchronous motion occurs as the result of interaction of all elements of the system, e.g. coupled synchronized oscillators, cooperative systems.

- In the second case, it is supposed that one object in the multi-composed system is more powerful than the others and its motion can be considered as independent of the motion of the other objects. Therefore the resulting synchronous motion is predetermined by this dominant independent system, e.g. masterslave systems, coordinated system.

From the control point of view the controlled synchronization problem is the most interesting, i.e. how to design a controller and/or interconnections that guarantee synchronization of the multi-composed system with respect to a certain desired func-

tional. The design of the control input and/or interconnections is mainly based on the feedback of the variables or signals that define the desired synchronous behavior.

\subsection{Synchronization in robotic systems}

Robot manipulators are widely used in production processes where high flexibility, manipulability and manoeuvrability are required. In tasks that cannot be carried 
out by a single robot, either because of the complexity of the task or limitations of the robot, the use of multi-robot systems working in external synchronization, e.g. master-slave and coordinated schemes, or mutual synchronization, e.g. cooperative schemes, has proved to be a good alternative. Coordinated and cooperative schemes are important illustrations of the same goal, where it is desired that two or more robot systems, either identical or different, work in synchrony (Brunt 1998), (Liu et al. 1997), (Liu et al. 1999). This can be formulated as a control problem that implies the design of suitable controllers to achieve the required synchronous motion.

In synchronization of robotic systems there exist several fundamental problems. First, a functional with respect to which the desired synchronization goal is described, has to be formulated. For this the type of robots and the variables of interest have to be taken into account. For robot synchronization the functionals can be defined as the norm of the difference between the variables of interest e.g. positions, velocities. Second, the couplings or interconnections and the feedback controllers to ensure the synchronous behavior have to be designed. The interconnections between the robots can be the feedback of the difference between the variables of interest. Finally conditions to guarantee the synchronization goal have to be determined.

The problem of synchronization of robotic systems seems to be a straightforward extension of classical tracking controllers, however it implies challenges that are not considered in the design of tracking controllers. The interconnections (interactions) between the robots imply control problems that are not considered in classical tracking controllers. However the interconnections cannot be neglected since they are precisely what determine the synchronized behavior and therefore the synchronization functional. The interconnections between the robots generate the flow of information necessary to guarantee the synchronous behavior. Most of the tracking controllers are only based on the signals of the controlled system, i.e. the desired and controlled position, velocity and acceleration. Therefore any external signal, like the signals due to couplings, is considered as a disturbance and its effects are supposed to be minimized or even cancelled by the controller.

Besides the fundamental problems of robot synchronization there exist other problems to be taken into account. Problems can arise because of the particular structure of the robots, such as type of joints (rigid or elastic), kinematic pairs (prismatic, rotational, etc.), transmission elements (gears, belts). Furthermore available equipment, for instance position, velocity and acceleration measuring capabilities, noise in the measurements and time delays, might cause other problems in robot synchronization. Some of the frequently encountered problems in robot synchronization are briefly discussed in the following sections.

Note that there are many other problems, such as underactuation and redundancy. However these problems are beyond the scope of this thesis. When redundant robot manipulators are considered the excess of actuated joints with respect to the degrees of freedom can be used to optimize certain functionals, avoid singularities, payload distribution, etc.. For this purpose synchronization schemes between the redundant joints can be used, however those synchronization schemes are different from the joint coordinate schemes considered in this thesis. On the other hand, for underactuated manipulators there are less actuated joints than degrees of freedom. In this situation the movement of the non-actuated joints is subject to holonomic or non-holonomic constraints. These constraints establish relations between the actuated and nonactuated joints, such that they can be considered as being synchronized, with the 
constraints as synchronization functionals. However, because there is no actuation the synchronization behavior of the non-actuated joints is achieved by the design of the robot and its own dynamics, therefore it is in fact self-synchronization.

We now briefly discuss some of the frequently encountered problems in robot synchronization.

\subsubsection{Velocity and acceleration measurements}

The controlled synchronization problem is further complicated by the fact that frequently only position measurements of the robots are available or reliable, due to a lack of velocity and acceleration measuring equipment or noise in the measurements. In practice, robot manipulators are equipped with high precision position sensors, such as optical encoders. Meanwhile new technologies have been designed for measuring angular velocities and accelerations, e.g. brushless AC motors with digital servo-drivers, microcontroller based measurements (Laopoulos and Papageorgiou 1996), digital processing (Kadhim et al. 1992), (Lygouras et al. 1998), linear accelerometers (Ovaska and Vliviita 1998), (Han et al. 2000). However, such technologies are not very common in applications yet. Therefore, very often the velocity measurements are obtained by means of tachometers, which are contaminated by noise. Moreover, velocity measuring equipment is frequently omitted due to the savings in cost, volume and weight that can be obtained. On the other hand acceleration measurements are indirectly obtained by pseudo-differentiation and filtering of the position and/or velocity direct measurements, such that the measurement noise is amplified and corrupts the acceleration measurements even farther.

\subsubsection{Joint flexibility}

Joint flexibility (also called joint elasticity) is caused by transmission elements such as harmonic drives, belts or long shafts and it can be modelled by considering the position and velocity of the motor rotor and the position and velocity of the link (De Luca and Tomei 1996), (Book 1984), (Spong 1987). Therefore the model of a joint flexible robot has twice the dimension of an equivalent rigid robot, and thus the controllers for flexible joint robots are more complex than those for rigid joint robots. It has been shown that joint flexibility considerably affects the performance of robot manipulators since it is major source of oscillatory behavior (Good et al. 1985). This means that, to improve the performance of robot manipulators, joint flexibility has to be taken into account in the modelling and control of such systems.

\subsubsection{Friction Phenomena}

Friction phenomena play an important role in control of robot manipulators. In high performance robotic systems, friction can severely deteriorate the performance. Bad compensation of the friction phenomena generates oscillatory behavior like limit cycles or stick-slip oscillations, introduces tracking errors, and in some cases can generate instability of the system (Armstrong-Hélouvry 1993), (Olsson and Åström 2001).

There is a great variety of friction models proposed in literature (Armstrong-Hélouvry et al. 1994), (Olsson et al. 1998), and each can be classified with respect to their detail in describing surface contact properties occurring on a microscopic and macroscopic level. Which model is more suitable for modelling and control purposes depends on 
the physical friction phenomena observed in the system such as stiction, Stribeck curve effects, viscous friction, etc. and on the velocity regime in which the system is suppose to work, i.e. slow, medium, or high speed. One major limitation in modelling friction phenomena is the complexity of the models and the drawback for parameter identification and control that it implies.

\subsection{Problem formulation}

The problem of synchronization of robot systems can be very wide depending on the kind of robots and their structural and measuring limitations, not to mention the possible synchronization goals. Therefore, this thesis is restricted to rigid and flexible rotational joint robots and internal or mutual synchronization (cooperative scheme) and external synchronization (coordinated and master-slave schemes).

The robots considered are fully actuated, i.e. the number of actuators is equal to the number of joints. It is also assumed that all the robots in the synchronization system have the same number of joints and equivalent joint work spaces, i.e. any possible configuration of a given robot in the system can be achieved by any other robot in the system. It does not imply that the robots are identical in their physical parameters, such as masses, inertias, etc.

Based on the robot manipulator structure described above and the possible synchronization schemes, the synchronizing problems addressed here can be formulated as follows.

\subsubsection{External synchronization of rigid joint robots}

Consider a multi-robot system formed by two or more rigid joint robots, such that the motion of one of the robots is independent of the other ones. This dominant robot will be referred to as the master robot. The master robot is driven by a controller already designed and not relevant for the synchronization goal. In the ideal case the controller ensures convergence of the master robot angular positions and velocities to a given desired trajectory. Then the goal is to design interconnections and feedback controllers for the non-dominant robots, hereafter referred to as slaves, such that their positions and velocities synchronize to those of the master robot. For the design of the slave interconnections and controllers it is assumed that only the master and slave angular positions for all joints are available for measurement. Furthermore only the dynamic model of the slave robot is assumed to be known, which complicates the reconstruction of the master angular velocity and acceleration since the master robot dynamic model is unknown.

Notice that the goal is to follow the trajectories of the master robot and not the desired trajectories of it, since these might not be achieved because of noise, parametric uncertainty or unmodelled dynamics of the master robot, like friction, unknown loads, etc.

Figure 1.1 shows a schematic representation of the external synchronization problem for the rigid joint robot case. The subindices $m, s$ and $d$ refer to the master robot, slave robot, and desired master robot trajectory respectively. The variables $q, \dot{q}$ represent the angular position and velocity of the joints, and $\tau$ denotes the input torque to the robots. Note that only the master robot position $q_{m}$ is transmitted to the slave robots. 


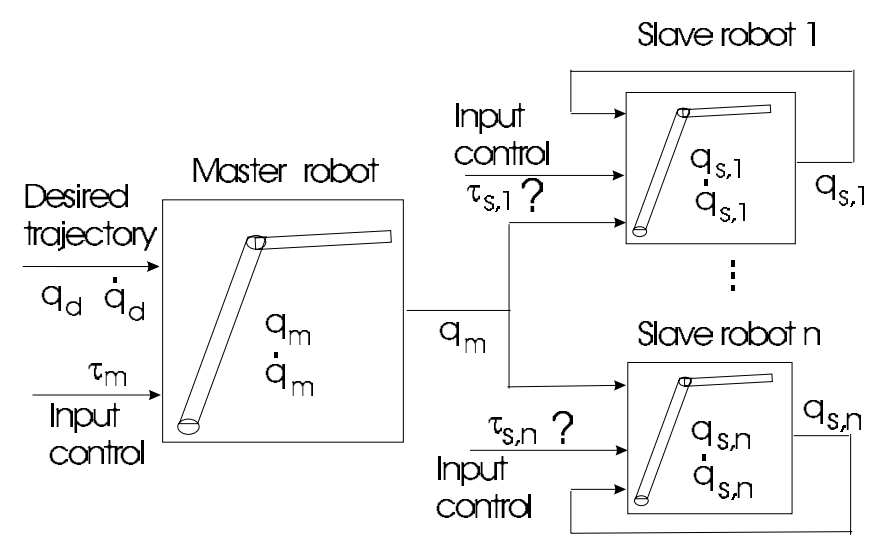

Figure 1.1: External synchronization of rigid joint robots.

\subsubsection{External synchronization of flexible joint robots}

For flexible joint robots the external synchronization control problem changes into the design of interconnections and feedback controllers for the slave robots such that their link angular positions and velocities synchronize to the master link angular positions and velocities. It is assumed that the only available information is the link angular position of the master and slave robots. Therefore the rotor angular positions, velocities and accelerations, as well as the angular velocity and acceleration of the link have to be all reconstructed. Furthermore the dynamic model of the master robot is assumed to be unknown, which complicates the reconstruction of its link and rotor velocities and accelerations. Joint flexibility plays an important role in how the interconnections between the robots are defined.

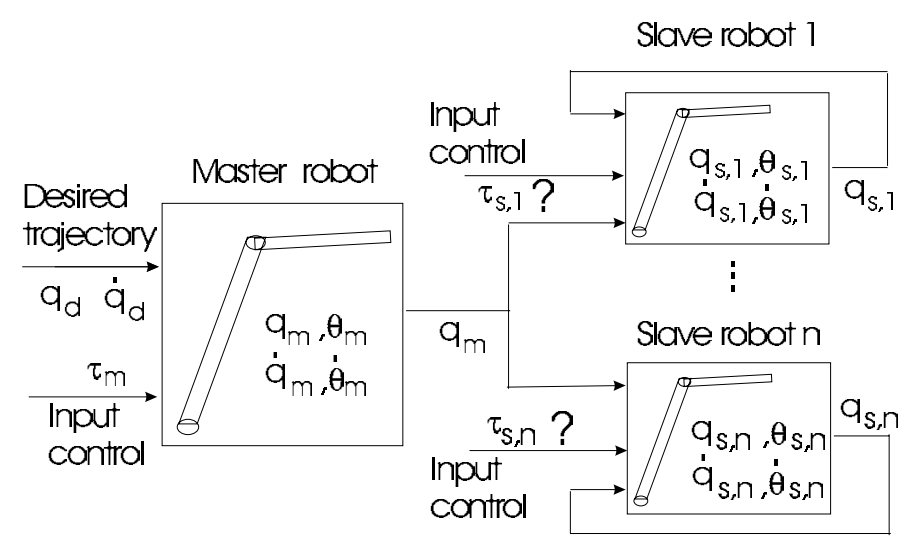

Figure 1.2: External synchronization of flexible joint robots.

Figure 1.2 shows a schematic representation of the external synchronization problem for the flexible joint robot case. The notation is the same as for external synchronization of rigid joint robots, but in this case also the angular position and velocity of the rotor of the motors, denoted by $\theta$ and $\dot{\theta}$, have to be considered. 


\subsubsection{Mutual (internal) synchronization of rigid joint robots}

External synchronization of robots restricts the resulting synchronization behavior since it is determined by the master robot, i.e. the dominant robot in the synchronization system. A more versatile system is obtained when interconnections from and to all the robots in the system, not only from the master to the slave robots, are considered. In that situation the synchronous behavior is the result of interactions between all the robots.

Consider a multi-robot system formed by two or more rigid joint robots such that there exists a common desired trajectory for all of them. Then the mutual synchronization control problem can be formulated as to design interconnections and feedback controllers for all the robots in the system, such that the angular positions and velocities of any robot in the system are synchronized with respect to the common desired trajectory and the angular positions and velocities of the other robots. It is assumed that only angular positions of all the robots are available for measurement. Also the dynamic models of all the robots are assumed to be known.

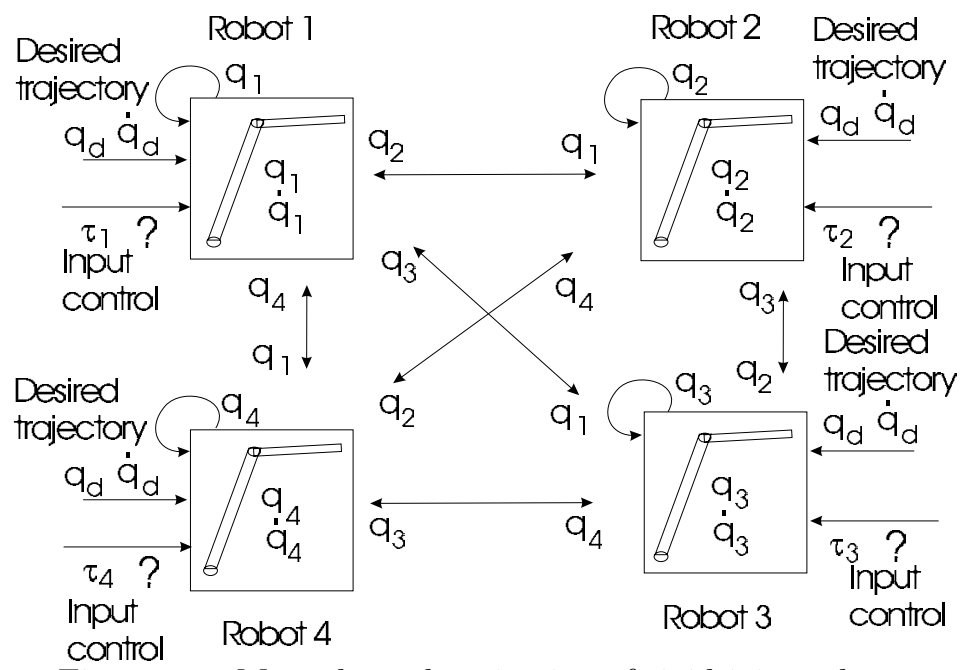

Figure 1.3: Mutual synchronization of rigid joint robots.

Figure 1.3 shows a schematic representation of the mutual synchronization problem for the rigid robot case. The notation is the same as for external synchronization of rigid joint robots. Note that in this problem the angular positions are transmitted from and to all the robots in the system. The input torques $\tau$ for all the robots must be designed to guarantee synchronous behavior of the system.

\subsection{Goals and main contributions of the thesis}

The main goal of this thesis is to prove analytically and validate experimentally that synchronization in multi-composed mechanical systems can be achieved in case of partial knowledge of the state vector of the systems, i.e. only positions are measured. For this purpose synchronization schemes based on interconnections between the systems, feedback controllers and observers are proposed. 
The role of the observers, which are model based, is to reconstruct the missing states of the systems that are used in the feedback controller. Of course, there are other alternative ways of estimating velocity and acceleration signals, like numerical differentiation or low pass filters (Kadhim et al. 1992), (Laopoulos and Papageorgiou 1996), (Lygouras et al. 1998), and in principle such alternatives could be used in the synchronization schemes developed here. These alternative techniques have the advantage of simplicity in implementation, but present a reduced bandwidth and in general there is not (or it is too difficult to determine) an analytical method to guarantee stability of the synchronizing scheme. The use of model-based observers is considered because one of the main goals is to provide a systematic way of proving and guaranteeing the synchronous behavior of the multi-composed system.

The feedback controller establishes the interconnections between the systems and determines the control action that guarantees the synchronous behavior of the multicomposed system. The feedback controllers considered here are based on a computed torque structure.

Because mechanical systems include a large variety of systems, and since it is impossible to address all of them, this thesis focuses on robot manipulators. Nonetheless the ideas developed here can be extended to other mechanical systems such as mobile robots, motors and generators.

To experimentally validate the proposed synchronization controllers a multi-composed robot system formed by two robot manipulators is considered as experimental setup. The robots in the experimental setup are industrial transposer robots designed by the Centre for Manufacturing Technology (CFT) Philips laboratory. These robots are installed at the Dynamics and Control Technology Laboratory at Eindhoven University of Technology. The robots have four degrees of freedom and are fully actuated by brushless DC servo-motors. The robots are identical in their structure and design, therefore they are represented by the same dynamic model. However, the robots are different in their parameters such as friction coefficients and inertias.

The main contributions of the thesis can be listed as follows.

- For external synchronization schemes (master-slave) two synchronization schemes are proposed, one for rigid joint robots and one for flexible joint robots. In both cases it is proved that synchronization can be achieved assuming only angular position measurements in all the interconnected robots. Moreover the synchronization scheme is decentralized and it does not require knowledge of the dynamic model of the master (dominant) system, but only the dynamic model of the slave system is required.

- For mutual (internal) synchronization only rigid joint robots are considered. It is proved that synchronization can be achieved when considering only angular position measurements in all the interconnected systems. The proposed synchronization controller is decentralized, but since there is not a dominant system, the dynamic model of all the interconnected systems are required.

- A simulation and experimental study shows the applicability and performance of the proposed synchronization controllers (external and mutual synchronization) for rigid joint robots. The experimental study also addresses the problems that are often encountered in robotic practical implementations. For external synchronization of flexible joint robots a simulation study shows the performance of the proposed synchronization controller. 


\subsection{Outline of the thesis}

Chapter 2 presents some basic material and results on which the thesis is based. In Section 2.1 some mathematical tools and stability concepts used throughout the thesis are presented. The dynamic models of rigid and flexible joint robots are introduced in Section 2.2, including their most important properties. The experimental setup is introduced in Section 2.3, where a brief description of the robots and their dynamic models are presented.

Chapter 3 addresses the problem of external synchronization of rigid joint robots. The synchronization scheme formed by a feedback controller and model based observers is presented and the stability proof is developed. Simulation and experimental results on one degree of freedom systems are included to show the applicability and performance of the proposed controller. The main contribution of this chapter is a gain tuning procedure that ensures synchronization of the interconnected robot systems. Some of the results of this chapter have been presented in the articles (Rodriguez-Angeles and Nijmeijer 2001a), (Rodriguez-Angeles and Nijmeijer 2001b), (Rodriguez-Angeles et al. $2002 b)$.

The case of external synchronization for flexible joint robots is addressed in Chapter 4. The chapter starts by explaining the differences between rigid and flexible joint robots and the effects on the design of the synchronization scheme. The synchronization scheme for flexible joint robots and stability analysis are presented. The chapter includes a gain tuning procedure that guarantees synchronization of the interconnected

robot systems. Simulation results on one degree of freedom systems are included to show the viability of the controller. Results presented in this chapter have been reported in the articles (Rodriguez-Angeles and Nijmeijer 2002b), (Rodriguez-Angeles et al. $2002 c)$.

The problem of internal (mutual) synchronization of rigid robots is treated in Chapter 5. This chapter presents a general synchronization scheme for the case of mutual synchronization of rigid robots. The chapter includes a general procedure to choose the interconnections between the robots to guarantee synchronization of the multicomposed robot system. Simulation and experimental results on one degree of freedom systems are included to show the properties of the controller. Results of this chapter have been reported in the article (Rodriguez-Angeles and Nijmeijer 2002a)

Chapter 6 presents a simulation and experimental study that shows the applicability and performance of the synchronization schemes for rigid joint robots. Particular attention is given to practical problems that can be encountered at the moment of implementing the proposed synchronization schemes. The robots in the experimental setup have four degrees of freedom, such that the complexity in the implementation is higher than in the simulations and experiments included in Chapters 3 and 5.

Further extensions of the synchronization schemes designed here are discussed in Chapter 7. Some conclusions and recommendations related to synchronization in general and robot synchronization in particular are presented in Chapter 8 . 


\section{Chapter 2}

\section{Preliminaries}

This chapter presents some mathematical preliminaries about stability and Lyapunov theory that are used throughout the thesis. Some definitions on stability and Lyapunov stability are briefly presented. For a more detailed synthesis on dynamical systems and stability the reader is referred to (Khalil 1996) and (Sastry 1999). Proposition 2.23 is especially useful in proving uniform ultimate stability of a system, and it is the main support of the stability analysis throughout this thesis.

The dynamic model of the rigid joint robot and the flexible joint robot are presented in Section 2.2. Some structural properties of both kind of robots are also introduced. These properties are useful throughout the stability analysis.

The experimental setup considered in Chapters 3 and 5 is introduced in Section 2.3. The dynamic model and estimated parameters of the robots are presented together with a brief description of the experimental setup.

\subsection{Mathematical preliminaries and stability con- cepts}

\subsubsection{Basic definitions}

Norms Throughout the thesis vector norms are Euclidean and they are denoted by $\|\cdot\|$, while for matrices the induced norm $\|A\|=\sqrt{\lambda_{\max }\left(A^{T} A\right)}$ is employed, with $\lambda_{\max }(\cdot)$ the maximum eigenvalue. Moreover, for any positive definite matrix $A$ we denote by $A_{m}$ and $A_{M}$ its minimum and maximum eigenvalues respectively. For a basic introduction about norms and induced norms the reader is referred to (Desoer and Vidyasagar 1975) and (Khalil 1996).

The following result is useful in proving positive definitiveness of symmetric block matrices.

Lemma 2.1 (Johnson 1990) If $L \in \mathbb{R}^{m \times m}$ and $M \in \mathbb{R}^{n \times n}$ are given positive semidefinite matrices and $X \in \mathbb{R}^{m \times n}$, then the symmetric block matrix

$$
\chi_{L M}=\left[\begin{array}{ll}
L & X \\
X^{T} & M
\end{array}\right]
$$


is positive semidefinite if and only if there exists a matrix $C \in \mathbb{R}^{m \times n}$, such that $X=L^{1 / 2} C M^{1 / 2}$.

If $L$ and $M$ are positive definite, this criterion is equivalent to

$$
\left\|L^{-1 / 2} X M^{-1 / 2}\right\| \leq 1
$$

Moreover $\chi_{L M}$ is positive definite if and only if $L$ and $M$ are positive definite and

$$
\left\|L^{-1 / 2} X M^{-1 / 2}\right\|<1
$$

Definition 2.2 A functional is a real-valued function on a vector space $V$, usually of functions. The reason the term "functional" is used is because $V$ can be a function space, e.g.

$$
V=\{f:[0,1] \rightarrow \mathbb{R} \text { such that } f \text { is continous }\}
$$

in which case e.g. $T(f)=f(0), T(f)=\int_{0}^{1} f(s) d s$ are linear functionals on $V$.

Definition 2.3 Consider an autonomous smooth nonlinear dynamical system represented by the differential equation

$$
\dot{x}(t)=f(t, x(t))
$$

A point $x=x^{*}$ in the state space is said to be an equilibrium point of (2.3) if $f\left(t, x^{*}\right) \equiv 0$ for all $t \geq 0$. An equilibrium point $x^{*}$ has the property that for any $t \geq 0$ if the state of the system starts at $x^{*}$ it will remain at $x^{*}$ for all future time.

Definition 2.4 A sphere of radius $r$ around the origin is denoted by $B_{r}$ i.e.,

$$
B_{r}=\left\{x \in \mathbb{R}^{n} \mid\|x\|<r\right\}
$$

Definition 2.5 A function $f: \mathbb{R}^{n} \rightarrow \mathbb{R}^{m}$ is said to be continuous at a point $x$ if given any $\epsilon>0$ a constant $\delta>0$ exists such that

$$
\|x-y\|<\delta \Rightarrow\|f(x)-f(y)\|<\epsilon
$$

Definition 2.6 A function $f: \mathbb{R}^{n} \rightarrow \mathbb{R}^{m}$ is said to be piecewise continuous on a set $S$ if it is continuous on $S$ except for a finite number of points.

Definition 2.7 A function $f: \mathbb{R}^{n} \rightarrow \mathbb{R}^{m}$ is said to be uniformly continuous on a set $S$ if for all $x, y \in S$ and given an $\epsilon>0$ a constant $\delta>0$ exists (depending only on $\epsilon$ ) such that (2.4) holds.

Often uniform continuity of a function $f: \mathbb{R} \rightarrow \mathbb{R}$ can be verified by means of the following lemma.

Lemma 2.8 Consider a differentiable function $f: \mathbb{R} \rightarrow \mathbb{R}$. If a constant $M \in \mathbb{R}$ exist such that

$$
\sup _{x \in \mathbb{R}}\left|\frac{d f}{d x}(x)\right| \leq M
$$

then $f$ is uniformly continuous on $\mathbb{R}$. 
Definition 2.9 A vector-valued function $f: \mathbb{R}^{n} \times \mathbb{R} \rightarrow \mathbb{R}^{n}$ satisfies a Lipschitz condition in $x$, with Lipschitz constant $\gamma>0$, if for a given $x_{1}, x_{2}$ and uniformly in $t$,

$$
\left\|f\left(t, x_{1}\right)-f\left(t, x_{2}\right)\right\| \leq \gamma\left\|x_{1}-x_{2}\right\|
$$

If the Lipschitz condition (2.5) holds for all $x_{1}, x_{2} \in \mathbb{R}^{n}$ then the function $f(t)$ is referred to as globally Lipschitz and as locally Lipschitz when it holds only in a region of $\mathbb{R}^{n}$.

Definition 2.10 A continuous function $\alpha:[0, a) \rightarrow[0, \infty)$ is said to belong to class $\mathcal{K}(\alpha \in \mathcal{K})$ if it is strictly increasing and $\alpha(0)=0$.

Definition 2.11 A continuous function $\beta:[0, a) \times[0, \infty) \rightarrow[0, \infty)$ is said to belong to class $\mathcal{K} \mathcal{L}(\beta \in \mathcal{K} \mathcal{L})$ if for each fixed s the mapping $\beta(r, s)$ belongs to class $\mathcal{K}$ with respect to $r$, and if for each fixed $r$ the mapping $\beta(r, s)$ is decreasing with respect to $s$ and $\beta(r, s) \rightarrow 0$ as $s \rightarrow \infty$.

\subsubsection{Lyapunov Stability}

Consider the non-autonomous system described by

$$
\dot{x}=f(t, x)
$$

where $f: \mathbb{R}^{+} \times D \rightarrow \mathbb{R}^{n}$ is piecewise continuous on $\mathbb{R}^{+} \times D$ and locally Lipschitz in $x$ on $\mathbb{R}^{+} \times D$, and $D \subset \mathbb{R}^{n}$ is a domain that contains the origin $x=0$. We assume that the origin is an equilibrium point for (2.6).

For studying the stability of the equilibrium point $x=0$ we introduce the following notations and definitions.

Definition 2.12 The equilibrium point $x=0$ of (2.6) is said to be (locally) stable (in the sense of Lyapunov) if a positive constant $r>0$ exist such that for all $\left(t_{0}, x\left(t_{0}\right)\right) \in \mathbb{R}^{+} \times B_{r}$ a function $\alpha \in \mathcal{K}$ exist such that

$$
\|x(t)\| \leq \alpha\left(\left\|x\left(t_{0}\right)\right\|\right) \quad \forall t_{0} \geq 0, \forall x\left(t_{0}\right) \in B_{r}
$$

If the above bound holds for all $\left(t_{0}, x\left(t_{0}\right)\right) \in \mathbb{R}^{+} \times \mathbb{R}^{n}$, the origin is globally stable.

Definition 2.13 The equilibrium point $x=0$ of (2.6) is said to be

- (locally) asymptotically stable if a constant $r>0$ exist such that for all pairs $\left(t_{0}, x\left(t_{0}\right)\right) \in \mathbb{R}^{+} \times B_{r}$ a function $\beta \in \mathcal{K} \mathcal{L}$ exist such that

$$
\|x(t)\| \leq \beta\left(\left\|x\left(t_{0}\right)\right\|, t-t_{0}\right) \quad \forall t \geq t_{0} \geq 0, \forall x\left(t_{0}\right) \in B_{r}
$$

- semi-globally asymptotically stable if for each constant $r>0$ and for all pairs $\left(t_{0}, x\left(t_{0}\right)\right) \in \mathbb{R}^{+} \times B_{r}$ a function $\beta \in \mathcal{K} \mathcal{L}$ exist such that (2.8) holds.

- globally asymptotically stable (GAS) if a function $\beta \in \mathcal{K} \mathcal{L}$ exist such that for all pairs $\left(t_{0}, x\left(t_{0}\right)\right) \in \mathbb{R}^{+} \times \mathbb{R}^{n}$ (2.8) holds. 
Definition 2.14 The equilibrium point $x=0$ of (2.6) is said to be (locally) exponentially stable if it is (locally) asymptotically stable and (2.8) is satisfied with

$$
\beta(r, s)=k r e^{-\gamma s} \quad k>0, r>0, \gamma>0
$$

In a similar way we can define the equilibrium point $x=0$ of (2.6) to be semi-globally exponentially stable and globally exponentially stable (GES).

Definition 2.15 The equilibrium point $x=0$ of (2.6) is said to be uniformly stable if a positive constant $r>0$ and $\alpha \in \mathcal{K}$ exist, both independent of $t_{0}$, such that

$$
\|x(t)\| \leq \alpha\left(\left\|x\left(t_{0}\right)\right\|\right) \quad \forall t \geq t_{0} \geq 0, \forall x\left(t_{0}\right) \in B_{r}
$$

If the above bound holds for all $\left(t_{0}, x\left(t_{0}\right)\right) \in \mathbb{R}^{+} \times \mathbb{R}^{n}$, the origin is globally uniformly stable.

Definition 2.16 The equilibrium point $x=0$ of (2.6) is said to be

- (locally) uniformly asymptotically stable if a constant $r>0$ and a function $\beta \in \mathcal{K} \mathcal{L}$ exist, both independent of $t_{0}$, such that

$$
\|x(t)\| \leq \beta\left(\left\|x\left(t_{0}\right)\right\|, t-t_{0}\right) \quad \forall t \geq t_{0} \geq 0, \forall x\left(t_{0}\right) \in B_{r}
$$

- semi-globally uniformly asymptotically stable if for each constant $r>0$ and for all $\left(t_{0}, x\left(t_{0}\right)\right) \in \mathbb{R}^{+} \times B_{r}$ a function $\beta \in \mathcal{K} \mathcal{L}$ exist such that (2.9) holds.

- globally uniformly asymptotically stable (GUAS) if a function $\beta \in \mathcal{K} \mathcal{L}$ exist such that for all $\left(t_{0}, x\left(t_{0}\right)\right) \in \mathbb{R}^{+} \times \mathbb{R}^{n}$ (2.9) holds.

Definition 2.17 The equilibrium point $x=0$ of (2.6) is said to be (locally) uniformly exponentially stable / semi-globally uniformly exponentially stable / globally uniformly exponentially stable (GUES) if it is (locally) uniformly asymptotically stable / semi-globally uniformly asymptotically stable / globally uniformly asymptotically stable and (2.9) is satisfied with

$$
\beta(r, s)=k r e^{-\gamma s} \quad k>0, r>0, \gamma>0 .
$$

To prove stability properties of a system many methods are based on what is called Lyapunov functions, and one of the most common theorem is Lyapunov's stability theorem, see (Khalil 1996).

Theorem 2.18 Let $x=0$ be an equilibrium point for $(2.6)$ and $D \subset \mathbb{R}^{n}$ be a domain containing $x=0$. Let $V: D \rightarrow \mathbb{R}^{n}$ be a continuously differentiable function, such that

$$
\begin{array}{ll}
V(0)=0 & \text { and } \quad V(x)>0 \quad \text { in } \quad D-\{0\} \\
\dot{V}(x) \leq 0 & \text { in } \quad D
\end{array}
$$

Then $x=0$ is stable. Moreover, if

$$
\dot{V}(x)<0 \quad \text { in } \quad D-\{0\}
$$

then $x=0$ is asymptotically stable. 
Notice that for asymptotic stability it is required that $\dot{V}(x)<0$ in $D-\{0\}$. However, there are some auxiliary theorems that allow to conclude asymptotic stability when $\dot{V}(x) \leq 0$. For autonomous systems it is still possible to prove asymptotic stability when $\dot{\vec{V}}(x) \leq 0$ by considering LaSalle's Theorem, while for non-autonomous systems Barbalat's Lemma is useful in proving asymptotic stability.

Theorem 2.19 LaSalle's Theorem: Given the system (2.6) suppose that a Lypaunov function candidate $V$ is found such that, along the solution trajectories

$$
\dot{V} \leq 0
$$

Then (2.6) is asymptotically stable if $\dot{V}$ does not vanish along any solution of (2.6) other than the null solution, that is, the system (2.6) is asymptotically stable if the only solution of (2.6) satisfying

$$
\dot{V} \equiv 0
$$

is the null solution.

Lemma 2.20 Barbalat's Lemma: Let $\phi: \mathbb{R} \rightarrow \mathbb{R}$ be a uniformly continuous function on $[0, \infty)$. Suppose that $\lim _{t \rightarrow \infty} \int_{0}^{t} \phi(\tau) d \tau$ exists and is finite. Then,

$$
\phi(t) \rightarrow 0 \quad \text { as } \quad t \rightarrow \infty
$$

\subsubsection{Stability of perturbed systems}

Consider the system

$$
\dot{x}=f(t, x)+g(t, x)
$$

where $f:[0, \infty) \times D \rightarrow \mathbb{R}^{n}$ and $g:[0, \infty) \times D \rightarrow \mathbb{R}^{n}$ are piecewise continuous in $t$ and locally Lipschitz in $x$ on $[0, \infty) \times D$, and $D \subset \mathbb{R}^{n}$ is a domain that contains the origin $x=0$. We think of this system as a perturbation of the nominal system (2.6). The perturbation term $g(t, x)$ could result from modelling errors or uncertainties and disturbances which exist in any realistic problem. In a typical situation, we do not know $g(t, x)$ but we might know some information about it, like knowing an upper bound on $\|g(t, x)\|$.

Assume that the origin $x=0$ is an exponentially stable equilibrium point for the nominal system (2.6). If the perturbation vanishes at the origin, i.e. $g(t, 0)=0$ for all $t$, then there exist conditions on $g(t, x)$ to prove that the origin is still an exponential stable equilibrium point for the perturbed system (2.12), see ((Khalil 1996), Theorem $5.1)$.

In the more general case we do not know that $g(t, 0)=0$. Then the origin $x=0$ may not be an equilibrium point of the perturbed system (2.12). The best we can hope is that if the perturbation term $g(t, x)$ is small in some sense, then $x(t)$ will be ultimately bounded by a small bound; that is, $\|x(t)\|$ will be small for sufficient large $t$. This brings in the concept of ultimate boundedness. 
Definition 2.21 The solutions of $\dot{x}=f(t, x)$ are said to be uniformly ultimately bounded if there exist positive constants $b$ and $c$ such that for every $\alpha \in(0, c)$ there is a positive constant $T=T(\alpha)$ such that

$$
\left\|x\left(t_{0}\right)\right\|<\alpha \Rightarrow\|x(t)\| \leq b, \quad \forall \quad t \geq t_{0}+T
$$

The solutions of $\dot{x}=f(t, x)$ are said to be globally uniformly ultimately bounded if (2.13) holds for arbitrarily large $\alpha$. Uniform ultimate boundedness is usually referred to as practical stability. The constant $b$ in (2.13) is referred to as the ultimate bound.

The following result supports the stability analysis that is carried out in this thesis. This result is a modified version of a theorem by (Chen and Leitmann 1987), see also (Berghuis and Nijmeijer 1994). It states that a system is uniformly ultimately bounded if it has a Lyapunov function whose time-derivative is negative definite in an annulus of a certain width around the origin.

Lemma 2.22 (Berghuis and Nijmeijer 1994) Consider the following function $g: \mathbb{R} \rightarrow$ $\mathbb{R}$

$$
g(y)=\alpha_{0}-\alpha_{1} y+\alpha_{2} y^{2}, \quad y \in \mathbb{R}^{+}
$$

where $\alpha_{i}>0, i=0,1,2$. Then $g(y)<0$ if $y_{1}<y<y_{2}$, where

$$
y_{1}=\frac{\alpha_{1}-\sqrt{\left(\alpha_{1}^{2}-4 \alpha_{2} \alpha_{0}\right)}}{2 \alpha_{2}}, \quad y_{2}=\frac{\alpha_{1}+\sqrt{\left(\alpha_{1}^{2}-4 \alpha_{2} \alpha_{0}\right)}}{2 \alpha_{2}}
$$

Proposition 2.23 (Chen and Leitmann 1987) Let $x(t) \in \mathbb{R}^{n}$ be the solution of the differential equation

$$
\dot{x}(t)=\vartheta(t, x(t))
$$

with $\vartheta(t, x(t))$ Lipschitz and initial condition $x\left(t_{0}\right)=x_{0}$, and assume there exists a function $V(x(t), t)$ that satisfies

$$
\begin{gathered}
P_{m}\|x(t)\|^{2} \leq V(x(t), t) \leq P_{M}\|x(t)\|^{2} \\
\dot{V}(x(t), t) \leq\|x(t)\| \cdot g(\|x(t)\|)<0 \quad \text { for all } \quad y_{1}<\|x(t)\|<y_{2}
\end{gathered}
$$

with $P_{m}$ and $P_{M}$ positive constants, $g(\cdot)$ as in (2.14), and $y_{1}, y_{2}$ as in (2.15). Define $\delta:=\sqrt{P_{m}^{-1} P_{M}}$. If $y_{2}>\delta y_{1}$, then $x(t)$ is locally uniformly ultimately bounded, that is, given $d_{m}=\delta y_{1}$, there exists $d \in\left(d_{m}, y_{2}\right)$ such that

$$
\left\|x_{0}\right\| \leq r \Rightarrow\|x(t)\| \leq d \quad \text { for all } \quad t \geq t_{0}+T(d, r)
$$

where

$$
T(d, r)=\left\{\begin{array}{cc}
0 & r \leq R \\
\frac{P_{M} r^{2}-P_{m} R^{2}}{-\alpha_{0} R+\alpha_{1} R^{2}-\alpha_{2} R^{3}} & R<r<\delta^{-1} y_{2}
\end{array}\right.
$$

and $R=\delta^{-1} d$. 


\subsection{Dynamic models of robot manipulators}

In this section dynamic models of rigid and flexible joint robots are introduced. Many ways exist to develop such models. For instance one can use the Lagrangian or Hamiltonian formulation of classical mechanics (Goldstein 1980), (Book 1984), which lead to a description based on differential equations. A different approach, based on a network representation of physical systems, is provided by the bond-graph technique (Breedveld et al. 1991), (Stramigioli 1998), whose multi-domain character is very useful in mechatronics. In this thesis the considered dynamic models are based on the Lagrangian approach. The resulting Lagrangian models possess structural properties due to the mechanics of the robots. The structural properties of the dynamical models that are used in this thesis are briefly listed in Section 2.2.3.

Section 2.2.4 gives a brief introduction into friction phenomena in robot manipulators. Particular attention is given to the friction phenomena since it greatly affects the performance of the robots. Although in some cases friction phenomena can straightforwardly be compensated, in general friction phenomena require special treatment.

\subsubsection{Rigid joint robots}

Consider a rigid joint robot with $n$ joints, i.e. with joint coordinates $q \in \mathbb{R}^{n}$. Assume that all the joints are rotational and fully actuated. The kinetic energy of the robot is given by $T(q, \dot{q})=\frac{1}{2} \dot{q}^{T} M(q) \dot{q}$, with $M(q) \in \mathbb{R}^{n \times n}$ the symmetric, positive definite inertia matrix, and the potential energy due to gravity is denoted by $U(q)$. Hence, applying the Euler-Lagrange formalism (Goldstein 1980), (Spong and Vidyasagar 1989) the dynamic model of the rigid joint robot is given by

$$
M(q) \ddot{q}+C(q, \dot{q}) \dot{q}+g(q)=\tau
$$

where $g(q)=\frac{\partial}{\partial q} U(q) \in \mathbb{R}^{n}$ denotes the gravity forces, $C(q, \dot{q}) \dot{q} \in \mathbb{R}^{n}$ represents the Coriolis and centrifugal forces, and $\tau$ denotes the $[n \times 1]$ vector of torques.

The dynamic model (2.18) does not account for some dynamic effects such as disturbances or friction, therefore the model (2.18) is referred to as nominal and frictionless. In case that friction phenomena are considered, we assume that friction effects can be compensated separately from the synchronization controller action. Feedback or feedforward schemes can be considered in order to compensate for friction effects.

\subsubsection{Flexible joint robots}

Joint flexibility, also called joint elasticity, considerably affects the performance of robot manipulators (Good et al. 1985), since they are a major source of oscillatory behavior. This means that in order to improve the performance of robot manipulators, joint flexibility has to be taken into account in the modelling and control of such systems. Joint flexibility can be caused by transmission elements such as harmonic drives, belts, or long shafts, and can be modelled by considering the position and velocity of the motor rotor $\theta, \dot{\theta}$, and of the link $q, \dot{q}$. Thus, the order of the dynamic model for a flexible joint is twice that of a rigid joint. Consequently, the control laws proposed for flexible joint robots are more complex than those for rigid robots.

From the modelling point of view two dynamic models for the flexible joint robot have been considered. In (Book 1984) and (Tomei 1991) an extended model for flexible joint robots is presented, which includes the full nonlinear dynamic interactions among 
joint flexibilities and inertial properties of links and actuators. On the other hand, if it is assumed that the kinetic energy of the electrical actuators is due only to their own rotor spinning, then a reduced model is obtained (Spong 1987).

The dynamic model of a flexible joint robot can be obtained by extending the procedures already used for rigid robots (Book 1984). Consider a flexible joint robot, with $n$ rigid links, all joints being flexible, rotational, and actuated by electrical drives. Let $q \in \mathbb{R}^{n}$ be the link positions and $\theta \in \mathbb{R}^{n}$ be the rotor positions, as reflected through the gear ratios. The difference $q_{i}-\theta_{i}$ is the $i$-th joint deformation. In view of small deformations, elasticity is modelled by means of a linear spring. The rotors of the motors are modelled as balanced uniform bodies having their centre of mass on the rotation axis, so that the inertia matrix and the gravity term in the dynamic model are independent from the motor position $\theta$. From (Book 1984) it follows that the extended dynamic model for a flexible joint robot is given by

$$
\begin{aligned}
& M(q) \ddot{q}+B_{1}(q) \ddot{\theta}+N_{1}(q, \dot{q}, \dot{\theta})+K(q-\theta)=0 \\
& B_{1}(q) \ddot{q}+J \ddot{\theta}+C_{3}(q, \dot{q}) \dot{q}+K(\theta-q)+B_{v} \dot{\theta}=\tau \\
& N_{1}(q, \dot{q}, \dot{\theta})=C(q, \dot{q}) \dot{q}+C_{1}(q, \dot{\theta}) \dot{q}+C_{2}(q, \dot{q}) \dot{\theta}+g(q)
\end{aligned}
$$

where the symmetric positive definite inertia matrix $M(q) \in \mathbb{R}^{n \times n}$, the Coriolis and centrifugal term $C(q, \dot{q}) \dot{q} \in \mathbb{R}^{n}$, and the gravity term $g(q) \in \mathbb{R}^{n}$ are all related to the rigid links, $J \in \mathbb{R}^{n \times n}$ is the constant diagonal inertia matrix of the motors, $K \in \mathbb{R}^{n \times n}$ is the constant diagonal matrix of the joint stiffness, $B_{v} \in \mathbb{R}^{n \times n}$ is the diagonal positive definite viscous friction coefficient matrix, and $\tau(\cdot)$ is the $n$-vector of torques supplied by the motors. The matrices $B_{1}(q), C_{1}(q, \dot{\theta}), C_{2}(q, \dot{q})$, and $C_{3}(q, \dot{q}) \in \mathbb{R}^{n \times n}$ represent coupling effects between the link and rotor at inertial and centrifugal level. Assuming that the motion of the rotors can be considered as pure rotations with respect to an inertial frame, the kinetic energy of each rotor is due to its own spinning. Therefore, the inertial and centrifugal coupling between links and rotors can be neglected and a reduced dynamic model is obtained, see (Spong 1987). Following (Spong 1987) and the Lagrangian approach (De Luca and Tomei 1996), it follows that the flexible joint robot dynamics can be modelled as

$$
\begin{aligned}
& M(q) \ddot{q}+N(q, \dot{q})+K(q-\theta)=0 \\
& J \ddot{\theta}+K(\theta-q)+B_{v} \dot{\theta}=\tau \\
& N(q, \dot{q})=C(q, \dot{q}) \dot{q}+g(q)
\end{aligned}
$$

Notice that the extended (2.19 - 2.21) and reduced (2.22 - 2.24) dynamic models only account for static viscous friction phenomena. For flexible joint robots the viscous friction induces damping in the dynamic model, such that the stability properties of the proposed synchronization scheme can be proved. Other kinds of friction phenomena can be considered in the models $(2.19-2.21)$ and $(2.22-2.24)$, and as in the rigid joint robots friction effects are assumed to be separately compensated from the synchronization control action.

The reduced model $(2.22-2.24)$ satisfies the conditions for full state linearization and decoupling via static state feedback. Meanwhile it has been proved (De Luca and Lanari 1995) that the extended model (2.19 - 2.21) is fully linearizable and decouplable via dynamic state feedback and a general algorithm is proposed in (De Luca and Lucibello 1998). In (De Luca 1998) the feedback linearization techniques are applied to the case of mixed rigid/elastic joints. Nevertheless other kind of techniques like 
singular perturbation and robust control have been investigated to design controllers for rigid-link flexible-joint robots, see (Lozano and Brogliato 1992), (Qu 1995).

All the above mentioned controllers assume that all state variables are available for measurement, implying the presence of additional sensors in each joint. In practice this is difficult if not impossible to achieve. Besides the complexity in the implementation of measuring equipment, velocity measuring equipment is frequently omitted due to the savings in cost, volume, and weight that can be obtained. To overcome this problem numerical differentiation, filters and the design of observers have been considered, see Section 1.3.1. For flexible joint robots several observer designs have been proposed. In (Nicosia et al. 1988) a nonlinear observer based on pseudolinearization techniques has been proposed, a high gain observer is presented in (Jankovic 1995), and a semiglobal nonlinear observer is designed in (Nicosia and Tomei 1995). In (Lim et al. 1997), (Dixon et al. 2000) adaptive controllers based on backstepping and filters requiring only link and actuator position measurements have been proposed. In this thesis we proposed linearizing feedback controllers and model based observers based only in link position measurements.

\subsubsection{Properties of the dynamic model of the robots}

The dynamic model of the rigid and flexible joint robot given by (2.18) and (2.22, 2.23) have the following properties.

- The inertia matrix $M(q) \in \mathbb{R}^{n \times n}$ is symmetric and positive definite for all $q \in \mathbb{R}^{n}$. The rotor inertia matrix $J \in \mathbb{R}^{n \times n}$ is a diagonal constant positive definite matrix.

- If the matrix $C(q, \dot{q}) \in \mathbb{R}^{n \times n}$ is defined using the Christoffel symbols (Spong and Vidyasagar 1989), then the matrix $\dot{M}(q)-2 C(q, \dot{q})$ is skew symmetric, i.e.

$$
x^{T}(\dot{M}(q)-2 C(q, \dot{q})) x=0 \quad \text { for all } \quad x \in \mathbb{R}^{n}
$$

- In addition, for the previous choice of $C(q, \dot{q})$, it follows that

$$
C(q, \dot{q})=\left[\begin{array}{c}
\dot{q}^{T} C_{1}(q) \\
\vdots \\
\dot{q}^{T} C_{n}(q)
\end{array}\right]
$$

where $C_{i}(q) \in \mathbb{R}^{n \times n} i=1, \ldots, n$ are symmetric matrices (Craig 1988). It follows that for any scalar $\alpha$ and for all $q, x, y, z \in \mathbb{R}^{n}$

$$
\begin{gathered}
C(q, x) y=C(q, y) x \\
C(q, z+\alpha x) y=C(q, z) y+\alpha C(q, x) y
\end{gathered}
$$

- $M(q), C(q, \dot{q})$ and $g(q)$ are bounded with respect to $q$, with bound different from 0, (Lewis et al. 1993), so

$$
\begin{gathered}
0<M_{m} \leq\|M(q)\| \leq M_{M}, \quad\|g(q)\| \leq g_{M} \quad \text { for all } q \in \mathbb{R}^{n} \\
\|C(q, x)\| \leq C_{M}\|x\| \quad \text { for all } \quad q, x \in \mathbb{R}^{n}
\end{gathered}
$$

Note that the properties (2.27) and (2.29) are due to linear dependence of the Coriolis and centrifugal torques on the second argument. 
- The diagonal positive definite viscous friction coefficient matrix $B_{v} \in \mathbb{R}^{n \times n}$ is bounded, with bound different from 0, i.e.

$$
0<B_{v, m} \leq\left\|B_{v}\right\| \leq B_{v, M}
$$

- The dynamic model (2.22), (2.23) can be transformed into a linear system via static state feedback (Spong 1987). The relation from the new input to the output $q$ is given by $n$ independent chains of 4 integrators.

\subsubsection{Friction phenomena}

Various mathematical models have been proposed to describe a number of friction phenomena (Armstrong-Hélouvry et al. 1994), (Olsson et al. 1998). Which model is more suitable for modelling and control purposes depends on the physical friction phenomena observed in the system, such as stiction, Stribeck curve effects, viscous friction, and on the velocity regime in which the system is supposed to work, i.e. slow, medium or high speed. One major limitation in modelling friction phenomena is the complexity of the models and the drawback for parameter identification and control that it implies. Due to the complexity of the physical mechanism underlying friction, most models are of an empirical nature. Furthermore a distinction can be made between static and dynamic models depending on the inclusion of frictional memory. For static friction models, this friction memory is omitted, whereas for dynamic friction models this memory behavior is described with additional dynamics between velocity and the friction force.

Dynamic friction models are useful to describe stick-slip phenomena and presliding displacements, such as elastic and plastic deformations of the asperity junctions before macroscopic sliding. In dynamic friction models the idea is to introduce extra state variables (or internal states) that determine the level of friction that depends on velocity. The evolution in time of the state variables is governed by a set of differential equations. Often the introduced state variables can be given a physical interpretation, which depends on the physical mechanism that the friction model is supposed to describe, see (Haessig and Friedland 1991), (Dupont and Dunlap 1995), (Canudas de Wit et al. 1995), and (Bliman and Sorine 1995).

Static friction models are characterized by the absence of internal states, i.e. they do not increase the order of the system. Static friction phenomena include Coulomb, viscous and Stribeck effects. The static friction models are static maps from the relative velocity between the two contact surfaces to the friction force. Several models for static friction have been proposed in the literature, see (Armstrong-Hélouvry et al. 1994), (Olsson et al. 1998).

In general dynamic friction models are more complicated than the static models. The advantages of dynamic friction models over static models are important at very low velocities, where dynamic friction greatly affects the performance of the systems. However, the use of dynamic friction models is not justified for medium and high velocities. Therefore only static friction models are considered throughout this thesis. A major difficulty in static models is the discontinuity that the Coulomb friction represents. The discontinuity at zero velocity may lead to non-uniqueness of the solution of the equation of motion, and numerical problems if such a model is used in simulations. A way to deal with the Coulomb discontinuity is to use approximations based on tangent or exponential functions. In this thesis we consider an approximation based on exponential functions as follows. Consider the friction model proposed in 
(Hensen et al. 2000), where the torque $f(\dot{q})$ due to friction is modelled as

$$
f(\dot{q})=B_{v} \dot{q}+B_{f 1}\left(1-\frac{2}{1+e^{2 w_{1} \dot{q}}}\right)+B_{f 2}\left(1-\frac{2}{1+e^{2 w_{2} \dot{q}}}\right)
$$

where $B_{v}$ is the viscous friction coefficient and the remaining terms model the Coulomb and Stribeck friction effects. The coefficients $w_{1}, w_{2}$ determine the slope in the approximation of the sgn function in the Coulomb friction and the Stribeck curve. Figure 2.1 shows typical friction forces obtained with the model (2.30). The coefficients in the model (2.30) were set as the estimated friction parameters listed in Table 2.1. Because of the particular values of the parameters $B_{f 1}, B_{f 2}, w_{1}$ and $w_{2}$ Stribeck friction effects are not present in Figure 2.1. For more friction models and a detailed discussion about friction effects the reader is referred to (Armstrong-Hélouvry et al. 1994).
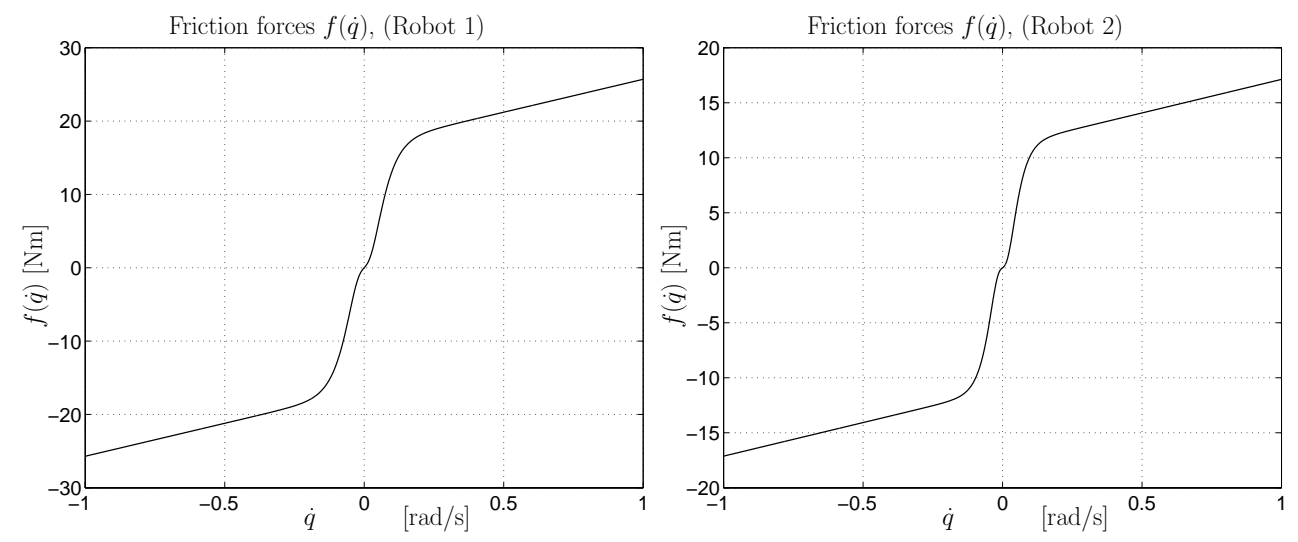

Figure 2.1: Continuous friction forces, static friction model (2.30)

\section{Friction compensation}

From the literature study (Armstrong-Hélouvry et al. 1994) a clear distinction can be made between non-model and model based friction compensation. In non-model based compensation the friction effects are compensated by using information of internal variables of the system, e.g. tracking errors, phase lag, etc., but without considering any friction model whatsoever. Examples of non-model based friction compensation controllers are the widely used stiff PD control and integral control, while impulsive control and dual mode control are less well known, see (Armstrong-Hélouvry 1991). On the other hand the model-based methods compensated the friction force by applying an equivalent control force in opposite direction. The most commonly used model-based compensation method is a fixed compensation based on an identified friction model. Other not so well know model based compensation techniques are adaptive control (Canudas de Wit and Lischinsky 1997) and learning control (Otten et al. 1997), which however are less used and have limited applicability.

From a control viewpoint friction compensation can be based on feedforward or feedback techniques. In the feedforward case the desired (commanded) frictional dependencies, such as velocities, position and/or acceleration, are used to compute the compensation control (command) force, whereas in the feedback setting the actual or instantaneous quantities are used. For most applications, only the instantaneous position is measured and consequently frictional dependencies such as velocity and acceleration must be reconstructed or estimated. 


\section{Friction phenomena in rigid joint robots}

The dynamic model for the rigid joint robot (2.18) does not account for friction phenomena. Nonetheless friction phenomena always appear in practical implementations and have to be compensated in order to improve the performance of the robots. Friction phenomena appear as an additive term in the dynamic model (2.18). To include friction phenomena in the dynamic model (2.18) the torques $\tau$ can be written as

$$
\tau=\tau_{e}-f(\dot{q})
$$

where $\tau_{e}$ is the $[n \times 1]$ vector of external or control torques and $f(\dot{q})$ corresponds to the torques due to friction effects. Consequently (2.18) becomes

$$
M(q) \ddot{q}+C(q, \dot{q}) \dot{q}+g(q)+f(\dot{q})=\tau_{e}
$$

As mentioned, there are several friction models, but throughout the thesis the friction forces $f(\dot{q})$ are considered to be modelled by $(2.30)$.

\section{Friction phenomena in flexible joint robots}

The dynamic model for the flexible joint robot $(2.22-2.24)$ only accounts for viscous friction effects. To include some other friction phenomena different from viscous friction, the torques $\tau$ in the dynamic model (2.22 - 2.24) can be written as

$$
\tau=\tau_{e}-f(\dot{q})
$$

where $\tau_{e}$ is the $[n \times 1]$ vector of external or control torques and $f(\dot{q})$ corresponds to the torques due to friction effects. Consequently $(2.22-2.24)$ become

$$
\begin{aligned}
& M(q) \ddot{q}+N(q, \dot{q})+K(q-\theta)=0 \\
& J \ddot{\theta}+K(\theta-q)+B_{v} \dot{\theta}+f(\dot{\theta})=\tau_{e} \\
& f(\dot{\theta})=B_{f 1}\left(1-\frac{2}{1+e^{2 w_{1} \dot{\theta}}}\right)+B_{f 2}\left(1-\frac{2}{1+e^{2 w_{2} \dot{\theta}}}\right)
\end{aligned}
$$

Remark 2.24 In the model (2.30) it is assumed that the friction is symmetric, and it is only a function of the joint velocity. However, in many robot applications it turns out that friction also exhibits some dependence on the joint position. Here, it is also assumed that the friction effects are decoupled with respect to the joint velocities, i.e. the friction effects on the $i$-th joint only depend on the $i$-th joint velocity.

\subsection{Experimental setup}

In order to experimentally validate the proposed synchronization controllers a multirobot system formed by two robots is considered. The robots in the experimental setup are industrial transposer robots designed by the Centre for Manufacturing Technology (CFT) Philips laboratory. These robots are installed at the Dynamics and Control Technology Laboratory at the Eindhoven University of Technology. The robots in the multi composed system are referred to as robot 1 and 2 .

The robots have four degrees of freedom and are fully actuated by brushless DC servomotors. The robots are equipped with encoders attached to the shaft of the motors with a resolution of 2000 PPR. Although the shaft of the motors and the corresponding links are connected by belts, the pair servomotor-link prove to be stiff 
enough to be considered as a rigid joint. The robots are identical in their structure and design, therefore they are represented by the same dynamic model. However the physical parameters such as inertias, friction coefficients, etc., are different for each robot. For implementation of the controllers and communication with the robots, the experimental setup is equipped with a DS1005 dSPACE system, with a processor PPC750, a clock of $480 \mathrm{MHz}$ and a bus clock of $80 \mathrm{MHz}$. Throughout the experiments the sampling frequency of the DS1005 dSPACE system was set to $2 \mathrm{kHz}$.

The robots have 4 degrees of freedom, but for the sake of clarity in the implementation in Chapters 3 and 5 only the middle link of the upper arm was used in the experiments. The other 3 degrees of freedom of the robots were locked such that only the middle links were able to move. Figure 2.2 shows the middle link of the robots. This link can rotate around its axis mounted in the based of the robot. Experiments with the four degrees of freedom of the robots are presented in Chapter 6 .

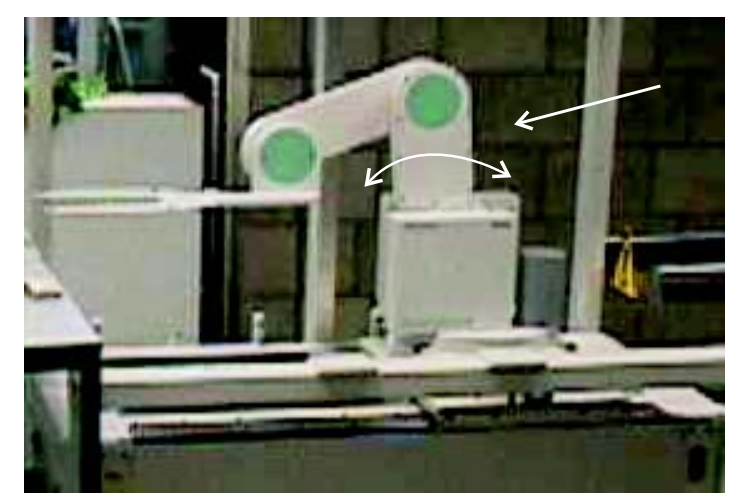

Figure 2.2: The transposer robot and its middle link.

Since in Chapters 3 and 5 only the medium link of the robots is considered, in this section the dynamic model for this link is presented. The dynamic model for the four degrees of freedom of the robot is presented in Appendix $\mathrm{H}$, together with a more detailed description of the transposer robots and the experimental setup.

In the position in which the 3 degrees of freedom of the transposer robot were locked, the model of the middle link, in both robots, can be described by $(i=1,2)$

$$
\begin{aligned}
& J_{i} \ddot{q}_{i}-g m_{i} \cos \left(-q_{i}-\alpha\right)+f_{i}\left(\dot{q}_{i}\right)=\tau_{i} \\
& f_{i}\left(\dot{q}_{i}\right)=B_{v, i} \dot{q}_{i}+B_{f 1, i}\left(1-\frac{2}{1+e^{2 w_{1, i} \dot{q}_{i}}}\right)+B_{f 2, i}\left(1-\frac{2}{1+e^{2 w_{2, i} \dot{q}_{i}}}\right)
\end{aligned}
$$

with $g=9.81 \mathrm{~m} / \mathrm{s}^{2}$ the acceleration due to the gravity. The torque $f_{i}\left(\dot{q}_{i}\right)$ models the friction with $B_{v, i}$ the viscous friction coefficient and the other terms representing the Coulomb and Stribeck friction effects.

The physical parameters of the dynamic model $(2.33,2.34)$ have been estimated using an extended Kalman filter in a similar way to the work presented in (Kostic et al. 2001). The input torque $\tau_{i}$ for collecting the data to run the Kalman filter was set as a $P$ controller, with a desired trajectory $q_{d}(t)$ of frequency $0.4 \mathrm{~Hz}$. The estimated parameters are listed in Table 2.1.

Figures 2.3 shows the estimated $\tau_{e, i}$ and measured torques $\tau_{i}$ for robots 1 and 2 . The estimated torque $\tau_{e, i}$ is obtained from the dynamic model $(2.33,2.34)$ and the estimated parameters in Table 2.1. It is worth to mention that the same methodology, 
controller and trajectories were used in both robots. However, robot 2 presents better fitting between the estimated and measured input control than robot 1 . Thus the dynamic model $(2.33,2.34)$ and the estimated parameters in Table 2.1 , are better representation of the dynamics of robot 2 than robot 1 . The differences in the estimated parameters may be due to weariness in the motors and transmission elements, even to better maintenance of robot 2. By simple inspection of the robots it is apparent that the robot 1 presents more weariness in the motors and transmission elements than robot 2 . The peaks in the estimated input control $\tau_{e, i}$ are due to friction effects, it is the robots present high frictional effects, particularly Coulomb friction.

\begin{tabular}{|c|c|c|c|}
\hline & $J_{i}\left[\mathrm{Kg} \cdot \mathrm{m}^{2}\right]$ & $m_{i}[\mathrm{Kg} \cdot \mathrm{m}]$ & $B_{v, i}\left[\mathrm{Kg} \cdot \mathrm{m}^{2} / \mathrm{s}\right]$ \\
\hline Robot 1 & 1.6627 & 2.8360 & 8.9815 \\
\hline Robot 2 & 1.7098 & 2.8027 & 6.1010 \\
\hline & $B_{f 1, i}\left[\mathrm{Kg} \cdot \mathrm{m}^{2} / \mathrm{s}^{2}\right]$ & $B_{f 2, i}\left[\mathrm{Kg} \cdot \mathrm{m}^{2} / \mathrm{s}^{2}\right]$ & $w_{1, i}$ \\
\hline Robot 1 & -10.6147 & 27.3291 & 27.6979 \\
\hline Robot 2 & -8.4152 & 19.4356 & 36.0641 \\
\hline & $w_{2, i}$ & $\alpha[\mathrm{rad}]$ & \\
\hline Robot 1 & 12.0224 & 2.5607 & \\
\hline Robot 2 & 16.2942 & 2.5607 & \\
\hline
\end{tabular}

Table 2.1: Estimated parameters for robot 1 and 2.
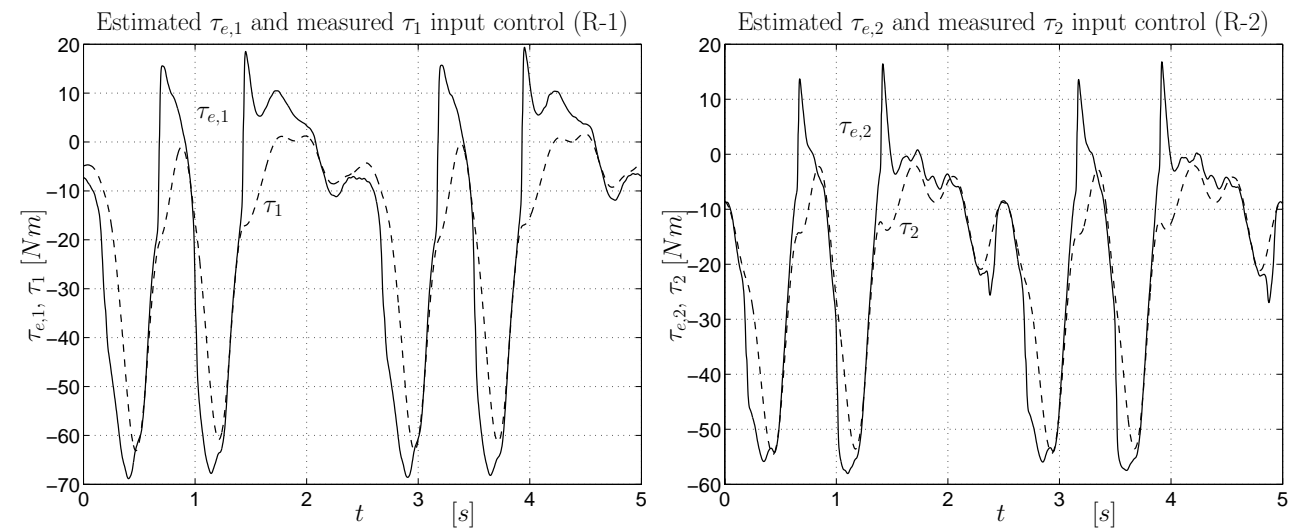

Figure 2.3: Estimated $\tau_{e, i}$ (solid) and measured torque $\tau_{i}$ (dashed) for robots 1,2.

In Chapter 3 there exists a desired reference $q_{d}(t)$, that is to be followed by the master robot, that corresponds to robot 1 , while robot 2 has the role of slave robot. Meanwhile in Chapter 5 a common desired trajectory $q_{d}(t)$ for both robots, robot 1 and 2 , is considered. For comparison the same desired trajectory $q_{d}(t)$ is considered in both Chapters 3 and 5 . The desired trajectory $q_{d}(t)$ is a series of harmonic functions with fundamental frequency $\omega$, which is given by

$$
q_{d}(t)=2.13+0.15 \sin (2 \pi \omega t)+0.05 \sin (4 \pi \omega t)-0.03 \sin (6 \pi \omega t)+0.02 \sin (8 \pi \omega t)
$$

The constant offset value 2.13 centers the amplitude of the oscillation of $q_{d}(t)$ in the middle of the span of the middle joint of the transposer robots. The other coefficients in (2.35) have been chosen arbitrarily, only taking into account the limits of the joint. 


\section{Chapter 3}

\section{External synchronization of rigid joint robots}

\subsection{Introduction}

In this chapter a novel approach for external position and velocity synchronization of two or more rigid joint robot manipulators under a master-slave scheme is presented. The external synchronization controller is only based on position measurements of the master and slave robots. Results in this chapter have been previously reported in the articles (Rodriguez-Angeles and Nijmeijer 2001a), (Rodriguez-Angeles and Nijmeijer 2001b), (Rodriguez-Angeles et al. 2002b).

The general setup is as follows: consider a multi-robot system that is formed by two (or more) fully actuated rigid joint robots with $n$ joints each. Assume that the motion of one of the robots is independent of the other robots. This robot is the dominant one and is referred to as the master robot, while the non-dominant robots are referred to as the slave robots. The master robot is driven by an input torque $\tau_{m}(\cdot)$, that, in the ideal case, ensures convergence of the joint angular positions and velocities $q_{m}, \dot{q}_{m} \in \mathbb{R}^{n}$ to a desired trajectory $q_{d}, \dot{q}_{d} \in \mathbb{R}^{n}$. However, the input torque $\tau_{m}$ is unknown, as well as the joint angular velocity and acceleration $\dot{q}_{m}, \ddot{q}_{m}$, and the master robot dynamic model. Under these assumptions, the goal is to design a control law $\tau_{s}(\cdot)$ for the slave robot, such that its joint angular position and velocity $q_{s}, \dot{q}_{s} \in \mathbb{R}^{n}$ synchronize with those of the master robot, i.e. $q_{m}, \dot{q}_{m}$. Also we assume that the slave joint velocities and accelerations $\dot{q}_{s}, \ddot{q}_{s}$ are not available for measurement. Therefore, the control law $\tau_{s}$ that is to be designed, can only depend on angular position measurements of both robots, i.e. $q_{m}, q_{s}$, and estimated values of the joint angular velocities and accelerations $\dot{q}_{m}, \ddot{q}_{m}, \dot{q}_{s}, \ddot{q}_{s}$. Figure 3.1 shows a schematic representation of the general setup for external synchronization of rigid joint robots.

Notice that the goal is to follow the trajectories of the master robot $q_{m}, \dot{q}_{m}$, and not the desired master robot trajectories $q_{d}, \dot{q}_{d}$, which may not be achieved because of noise, parametric uncertainty or unmodelled dynamics of the master robot, like unmodelled friction phenomena, unknown loads, etc.

Throughout this chapter and without loss of generality a multi-robot system formed by two rigid joint robots is considered. Hereafter the subindices $m, s$ distinguish between the master and slave robot. The cases of robot with and without friction 


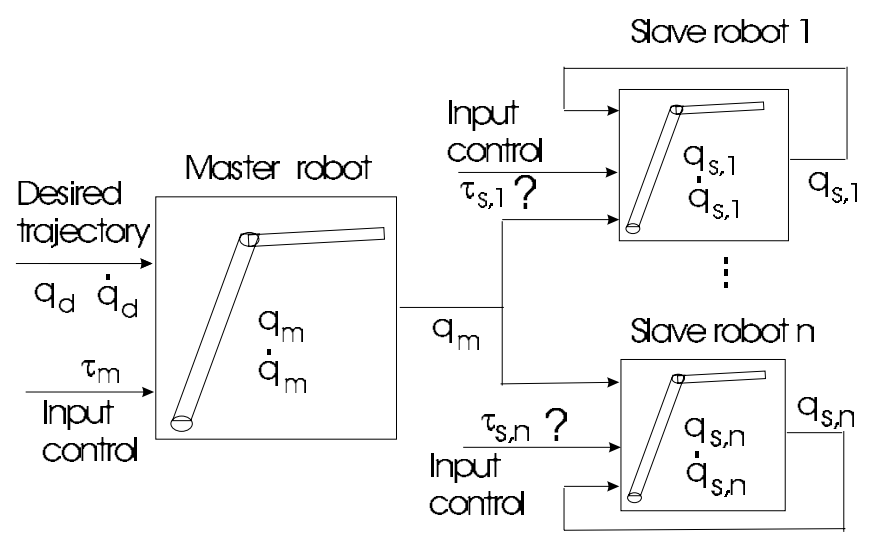

Figure 3.1: External synchronization of rigid joint robots.

effects are both addressed.

If friction effects are ideally compensated, then the robots can be considered as frictionless and can be modelled as (see Section 2.2.1)

$$
M_{i}\left(q_{i}\right) \ddot{q}_{i}+C_{i}\left(q_{i}, \dot{q}_{i}\right) \dot{q}_{i}+g_{i}\left(q_{i}\right)=\tau_{i}, \quad i=m, s
$$

while, when friction forces $f_{i}\left(\dot{q}_{i}\right)$ are considered the model changes to

$$
M_{i}\left(q_{i}\right) \ddot{q}_{i}+C_{i}\left(q_{i}, \dot{q}_{i}\right) \dot{q}_{i}+g_{i}\left(q_{i}\right)+f_{i}\left(\dot{q}_{i}\right)=\tau_{i}
$$

In this chapter the particular friction model $f_{i}\left(\dot{q}_{i}\right)$ introduced in Section 2.2.4 is considered

$$
f_{i}\left(\dot{q}_{i}\right)=B_{v, i} \dot{q}_{i}+B_{f 1, i}\left(1-\frac{2}{1+e^{2 w_{1, i} \dot{q}_{i}}}\right)+B_{f 2, i}\left(1-\frac{2}{1+e^{2 w_{2, i} \dot{q}_{i}}}\right)
$$

One of the problems in synchronization in general is to formulate the functionals that describe the synchronized behavior. In our particular problem we consider that synchronization occurs when the angular position and velocity of the master and slave robot coincide for all $t \geq 0$, or asymptotically for $t \rightarrow \infty$. Then one can introduce synchronization indices as the angular variables of interest

$$
J(q(t), \dot{q}(t))=\left[\begin{array}{ll}
q(t)^{T} & \dot{q}(t)^{T}
\end{array}\right]
$$

with the corresponding set of functionals

$$
f_{\text {sync }}\left(q_{m}, \dot{q}_{m}, q_{s}, \dot{q}_{s}\right)=\left\|J\left(q_{m}, \dot{q}_{m}\right)-J\left(q_{s}, \dot{q}_{s}\right)\right\|
$$

The chapter is organized as follows. A synchronization controller for frictionless robots, assuming full state measurements is presented in Section 3.2. Section 3.3 presents a modified synchronization controller formed by a feedback control and two nonlinear observers. The stability analysis of the synchronization closed loop system is given in Section 3.3.6. A gain tuning procedure for the observers and feedback controller gains is given in Section 3.4. Section 3.5 describes a friction compensation scheme of the particular friction phenomena modelled by (3.3). A simulation and experimental study in a one degree of freedom system is presented in Section 3.6. The chapter closes with some concluding remarks and discussion about the proposed synchronization controller, Section 3.7. 


\subsection{Synchronization controller based on state feed- back}

If the master and slave positions, velocities and accelerations are available for measurement, then master slave synchronization can be obtained by using some of the tracking controllers proposed in literature. For instance, consider the tracking controller proposed by Paden and Panja (1988) and take the master position $q_{m}$, velocity $\dot{q}_{m}$, and acceleration $\ddot{q}_{m}$ as desired trajectories for the slave robot. Then we propose a synchronization controller of the form

$$
\tau_{s}=M_{s}\left(q_{s}\right) \ddot{q}_{m}+C_{s}\left(q_{s}, \dot{q}_{s}\right) \dot{q}_{m}+g_{s}\left(q_{s}\right)-K_{d} \dot{e}-K_{p} e
$$

with the synchronization errors $e, \dot{e} \in \mathbb{R}^{n}$ defined by

$$
e:=q_{s}-q_{m}, \quad \dot{e}:=\dot{q}_{s}-\dot{q}_{m}
$$

$M_{s}\left(q_{s}\right), C_{s}\left(q_{s}, \dot{q}_{s}\right), g_{s}\left(q_{s}\right)$ are as in Section 2.2.1, and $K_{p}, K_{d} \in \mathbb{R}^{n \times n}$ are positive definite gain matrices.

Remark 3.1 Note that the synchronization functionals $f_{s y n c}\left(q_{m}, \dot{q}_{m}, q_{s}, \dot{q}_{s}\right)$, defined by (3.5), can be written in terms of the synchronization errors e, $\dot{e}$, i.e.

$$
f_{\text {sync }}\left(q_{m}, \dot{q}_{m}, q_{s}, \dot{q}_{s}\right)=\left\|\left[\begin{array}{ll}
e^{T} & \dot{e}^{T}
\end{array}\right]\right\|
$$

By using standard stability techniques, it can be proved that the controller (3.6) yields asymptotic synchronization between the master and slave robots.

Theorem 3.2 For any positive definite gain matrices $K_{p}, K_{d}$, the synchronization closed loop system formed by the slave robot (3.1) and the controller (3.6) is asymptotically stable.

Proof: From the properties of the Coriolis term (see Section 2.2.3) it follows that the synchronization closed loop system is given by

$$
M_{s}\left(q_{s}\right) \ddot{e}=-C_{s}\left(q_{s}, \dot{q}_{s}\right) \dot{e}-K_{d} \dot{e}-K_{p} e
$$

Take as a Lyapunov function

$$
V=\frac{1}{2} \dot{e}^{T} M_{s}\left(q_{s}\right) \dot{e}+\frac{1}{2} e^{T} K_{p} e
$$

then along the error dynamics (3.8) the time derivative of $V$ results in

$$
\dot{V}=-\dot{e}^{T} K_{d} \dot{e}
$$

Note that $\dot{V}$ is only a negative semi-definite function of the state. Nevertheless, by using stability theorems such as LaSalle's theorem (Theorem 2.19) and Barbalat's lemma (Lemma 2.20) it can be proved that the only invariant set that satisfies $\dot{V}=0$ along trajectories of the closed loop systems is $\{(e, \dot{e})=(0,0)\}$, see (Lewis et al. 1993). Therefore it can be concluded that the synchronization errors $e, \dot{e}$ are asymptotically stable for any positive definite matrices $K_{p}, K_{d}$, thus the slave robot synchronizes in position and velocity to the master robot. 


\subsection{Synchronization controller based on estimated variables}

As stated in the introduction, it is assumed that there is no access to $\left(\dot{q}_{m}, \ddot{q}_{m}\right)$ and $\left(\dot{q}_{s}, \ddot{q}_{s}\right)$, but only joint positions $q_{m}$ and $q_{s}$ can be measured. Therefore, the synchronization controller (3.6) cannot be implemented. As an alternative the synchronization controller can be formed by a feedback control law and two nonlinear model based observers. In this way the synchronization controller $\tau_{s}(\cdot)$ depends on position measurements $\left(q_{m}, q_{s}\right)$ and estimated values for the velocities $\left(\dot{q}_{m}, \dot{q}_{s}\right)$ and accelerations $\left(\ddot{q}_{m}, \ddot{q}_{s}\right)$.

\subsubsection{Feedback control law}

With the controller (3.6) in mind, and under the assumptions that the friction effects have been compensated, the estimated values are available, and the nonlinearities and parameters of the slave robot are known, we propose the controller $\tau_{s}$ for the slave robot as

$$
\tau_{s}=M_{s}\left(q_{s}\right) \widehat{\ddot{q}}_{m}+C_{s}\left(q_{s}, \widehat{\dot{q}}_{s}\right) \widehat{\dot{q}}_{m}+g_{s}\left(q_{s}\right)-K_{d} \widehat{\dot{e}}-K_{p} e
$$

were $\widehat{\dot{q}}_{s}, \widehat{\dot{e}}, \widehat{\dot{q}}_{m}, \widehat{\ddot{q}}_{m} \in \mathbb{R}^{n}$ represent the estimates of $\dot{q}_{s}, \dot{e}, \dot{q}_{m}$ and $\ddot{q}_{m}$ respectively, the synchronization errors $e, \dot{e} \in \mathbb{R}^{n}$ are defined by $(3.7), M_{s}\left(q_{s}\right), C_{s}\left(q_{s}, \widehat{\dot{q}}_{s}\right), g_{s}\left(q_{s}\right)$ are defined as in Section 2.2.1, and $K_{p}, K_{d} \in \mathbb{R}^{n \times n}$ are positive definite gain matrices.

\subsubsection{An observer for the synchronization errors $e, \dot{e}$}

Estimated values for the synchronization errors $e, \dot{e}(3.7)$ are denoted by $\widehat{e}, \widehat{\dot{e}}$; these estimated values are obtained by the full state nonlinear Luenberger observer

$$
\begin{aligned}
\frac{d}{d t} \widehat{e} & =\widehat{\dot{e}}+\Lambda_{1} \widetilde{e} \\
\frac{d}{d t} \widehat{\dot{e}} & =-M_{s}\left(q_{s}\right)^{-1}\left[C_{s}\left(q_{s}, \hat{\dot{q}}_{s}\right) \widehat{\dot{e}}+K_{d} \widehat{\dot{e}}+K_{p} \widehat{e}\right]+\Lambda_{2} \widetilde{e}
\end{aligned}
$$

where the estimated position and velocity synchronization errors $\widetilde{e}, \widetilde{e}$ are defined by

$$
\widetilde{e}:=e-\widehat{e}, \quad \widetilde{\dot{e}}:=\dot{e}-\widehat{\dot{e}}
$$

and $\Lambda_{1}, \Lambda_{2} \in \mathbb{R}^{n \times n}$ are positive definite gain matrices.

\subsubsection{An observer for the slave joint variables $q_{s}, \dot{q}_{s}$}

Lets $\widehat{q}_{s}, \widehat{\dot{q}}_{s}$ denote estimated values for $q_{s}, \dot{q}_{s}$. To compute these estimates, we propose the full state nonlinear observer

$$
\begin{aligned}
& \frac{d}{d t} \widehat{q}_{s}=\widehat{\dot{q}}_{s}+L_{p 1} \widetilde{q}_{s} \\
& \frac{d}{d t} \widehat{\dot{q}}_{s}=-M_{s}\left(q_{s}\right)^{-1}\left[C_{s}\left(q_{s}, \widehat{\dot{q}}_{s}\right) \widehat{\dot{e}}+K_{d} \widehat{\dot{e}}+K_{p} e\right]+L_{p 2} \widetilde{q}_{s}
\end{aligned}
$$

where the estimated position and velocity errors $\widetilde{q}_{s}$ and $\widetilde{\dot{q}}_{s}$ are defined by

$$
\widetilde{q}_{s}:=q_{s}-\widehat{q}_{s} \quad \widetilde{\dot{q}}_{s}:=\dot{q}_{s}-\widehat{\dot{q}}_{s}
$$

and $L_{p 1}, L_{p 2} \in \mathbb{R}^{n \times n}$ are positive definite gain matrices. 


\subsubsection{Estimated values for $\dot{q}_{m}, \ddot{q}_{m}$}

As stated, the master robot variables $\dot{q}_{m}, \ddot{q}_{m}$ are not available, therefore estimated values for $\dot{q}_{m}, \ddot{q}_{m}$ are used in $\tau_{s}(3.9)$. However, the master robot dynamic model is assumed to be unknown, then the variables $\dot{q}_{m}, \ddot{q}_{m}$ must be reconstructed based on information of the slave robot and the synchronization closed loop system. From (3.7) and the definition of the estimated variables $\widehat{e}, \widehat{\dot{e}}, \widehat{q}_{s}, \widehat{\dot{q}}_{s}$, we can consider that estimated values for $q_{m}, \dot{q}_{m}, \ddot{q}_{m}$ are given by

$$
\begin{aligned}
\widehat{q}_{m} & =\widehat{q}_{s}-\widehat{e} \\
\widehat{\dot{q}}_{m} & =\widehat{\dot{q}}_{s}-\widehat{\dot{e}} \\
\widehat{\ddot{q}}_{m} & =\frac{d}{d t}\left(\widehat{\dot{q}}_{s}-\widehat{\dot{e}}\right)
\end{aligned}
$$

from the definition of the observers (3.10), (3.12) it follows that

$$
\widehat{\ddot{q}}_{m}=-\left(M_{s}\left(q_{s}\right)^{-1} K_{p}+L_{p 2}\right) \widetilde{e}+L_{p 2} \widetilde{q}_{s}
$$

which gives a clear insight into how the master joint acceleration $\widehat{\ddot{q}}_{m}$ is reconstructed.

Remark 3.3 In the feedback control (3.9) and the observer (3.12) the available signal $e$ is used, instead of its estimate $\hat{e}$. This is done to take advantage of the available information, i.e. the position measurement $q_{s}$ and the synchronization position error $e$. As a result robustness and better stability and performance during transients are obtained.

Remark 3.4 Note that, in (3.10) and (3.11) the estimate for the synchronization error $\dot{e}$ is given by $\hat{\dot{e}}$, not by $\dot{\hat{e}}=\frac{d}{d t} \widehat{e}$. This definition introduces an extra correcting term in $\dot{\widetilde{e}}$, as it follows from (3.10), (3.11) that

$$
\dot{\widetilde{e}}=\dot{e}-\dot{\hat{e}}=\tilde{e}-\Lambda_{1} \widetilde{e}
$$

The term $\Lambda_{1} \widetilde{e}$ gives faster estimation performance, especially during transients, but it has some negative effects on noise sensitivity, since it amplifies noise measurements on $\widetilde{e}$.

The same can be said for observer (3.12) and the estimation position and velocity errors (3.13).

Figure 3.2 shows a schematic representation of the proposed synchronization controller for rigid joint robots.

\subsubsection{Synchronization closed loop error dynamics}

To simplify the synchronization closed loop error dynamics and the stability analysis the following assumptions on the gain matrices $K_{p}, K_{d}, L_{p 1}, L_{p 2}, \Lambda_{1}, \Lambda_{2}$ are considered.

Assumption 3.5 The gain matrices $\Lambda_{1}, \Lambda_{2}$ and $L_{p 1}, L_{p 2}$ satisfy

$$
\Lambda_{1}=L_{p 1}, \quad \Lambda_{2}=L_{p 2}
$$




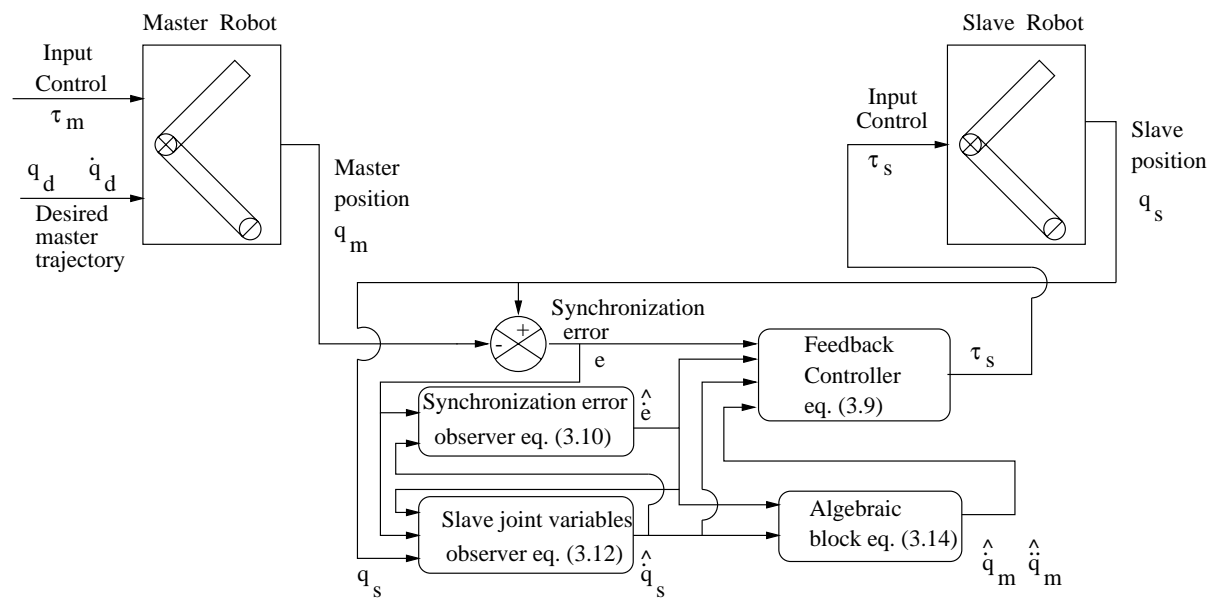

Figure 3.2: External synchronization of rigid joint robots.

Assumption 3.6 The gain matrices $K_{p}, K_{d}, L_{p 1}, L_{p 2}$ are symmetric.

Furthermore two coordinate transformations are introduced.

Lemma 3.7 Consider the synchronization errors $(e, \dot{e})$, the estimated synchronization errors $(\widetilde{e}, \widetilde{\dot{e}})$ and the estimated position and velocity errors $\left(\widetilde{q}_{s}, \widetilde{\dot{q}}_{s}\right)$, which are defined by (3.7), (3.11), and (3.13).

Introduce the coordinate transformation defined by

$$
\begin{aligned}
& \widetilde{q}_{m}:=\widetilde{e}-\widetilde{q}_{s} \\
& \dot{\widetilde{q}}_{m}:=\widetilde{\dot{e}}-\widetilde{\dot{q}}_{s}-L_{p 1} \widetilde{q}_{m} \\
& \dot{\widetilde{q}}_{s}:=\widetilde{\dot{q}}_{s}-L_{p 1} \widetilde{q}_{s}
\end{aligned}
$$

and

$$
\begin{gathered}
\bar{q}:=e-\widetilde{q}_{m} \\
\dot{\bar{q}}:=\dot{e}-\dot{\widetilde{q}}_{m}
\end{gathered}
$$

Define the vectors $x, y \in \mathbb{R}^{6 n}$ as

$$
\begin{aligned}
x^{T} & :=\left[\begin{array}{llllll}
\dot{e}^{T} & e^{T} & \tilde{\dot{e}}^{T} & \widetilde{e}^{T} & \tilde{\dot{q}}_{s}^{T} & \widetilde{q}_{s}^{T}
\end{array}\right] \\
y^{T} & :=\left[\begin{array}{llllll}
\dot{\bar{q}}^{T} & \bar{q}^{T} & \dot{\tilde{q}}_{m}^{T} & \widetilde{q}_{m}^{T} & \dot{\widetilde{q}}_{s}^{T} & \widetilde{q}_{s}^{T}
\end{array}\right]
\end{aligned}
$$

then $x$ and $y$ are related by

$$
x=T y
$$

where

$$
T=\left[\begin{array}{cccccc}
I & 0 & I & 0 & 0 & 0 \\
0 & I & 0 & I & 0 & 0 \\
0 & 0 & I & L_{p 1} & I & L_{p 1} \\
0 & 0 & 0 & I & 0 & I \\
0 & 0 & 0 & 0 & I & L_{p 1} \\
0 & 0 & 0 & 0 & 0 & I
\end{array}\right]
$$


Proof: The proof follows from the definition of the coordinate transformations.

Remark 3.8 Note that from the definition of the variables $\widetilde{q}_{m}, \dot{\widetilde{q}}_{m}$, (3.16), it follows that

$$
\widetilde{q}_{m}=\widehat{q}_{m}-q_{m}, \quad \dot{\widetilde{q}}_{m}=\dot{\widehat{q}}_{m}-\dot{q}_{m}
$$

therefore, $\widetilde{q}_{m}, \dot{\widetilde{q}}_{m}$ can be interpreted as the estimation error in the joint variables of the master robot $q_{m}, \dot{q}_{m}$.

Meanwhile, $\bar{q}, \dot{\bar{q}}$ satisfy

$$
\bar{q}=q_{s}-\widehat{q}_{m}, \quad \dot{\bar{q}}=\dot{q}_{s}-\dot{\widehat{q}}_{m}
$$

thus, they represent the error between the slave trajectories and estimated master trajectories, i.e. the practical errors that are fedback into the system. Note that $q_{m}$ is available for measurement and is used in the controller (3.9) and both observers (3.10).

In the new error coordinates, the synchronization closed loop error dynamics can be formulated as follows.

Lemma 3.9 Consider the synchronization closed loop system formed by the slave robot (3.1), the controller (3.9), and both observers (3.10), (3.12). Then, in the variables defined by (3.13), (3.16), and (3.17), the synchronization closed loop error dynamics are given by

$$
\begin{array}{r}
M_{s}\left(q_{s}\right) \ddot{\bar{q}}+C_{s}\left(q_{s}, \dot{q}_{s}\right) \dot{\bar{q}}+K_{d} \dot{\bar{q}}+K_{p} \bar{q}=C_{s}\left(q_{s}, \dot{\widetilde{q}}_{s}+L_{p 1} \widetilde{q}_{s}\right)\left(\dot{\bar{q}}-L_{p 1} \widetilde{q}_{m}\right) \\
+M_{s}\left(q_{s}\right) L_{p 1} \dot{\widetilde{q}}_{m}+C_{s}\left(q_{s}, \dot{q}_{s}\right) L_{p 1} \widetilde{q}_{m}+K_{d}\left(\dot{\widetilde{q}}_{s}+L_{p 1}\left(\widetilde{q}_{s}+\widetilde{q}_{m}\right)\right)-K_{p} \widetilde{q}_{m} \\
\ddot{\widetilde{q}}_{m}=-M_{s}\left(q_{s}\right)^{-1} K_{p}\left(\widetilde{q}_{m}+\widetilde{q}_{s}\right)-L_{p 1} \dot{\widetilde{q}}_{m}-L_{p 2} \widetilde{q}_{m}-\ddot{q}_{m} \\
\ddot{\widetilde{q}}_{s}=M_{s}\left(q_{s}\right)^{-1}\left[-K_{p}\left(\widetilde{q}_{m}+\widetilde{q}_{s}\right)+\left(C_{s}\left(q_{s}, \dot{\widetilde{q}}_{s}+L_{p 1} \widetilde{q}_{s}\right)-2 C_{s}\left(q_{s}, \dot{q}_{s}\right)\right)\right. \\
\left.\times\left(\dot{\widetilde{q}}_{s}+L_{p 1} \widetilde{q}_{s}\right)\right]-L_{p 1} \dot{\widetilde{q}}_{s}-L_{p 2}\left(\widetilde{q}_{m}+\widetilde{q}_{s}\right)
\end{array}
$$

Proof: See Appendix A.

Remark 3.10 Note that the master acceleration $\ddot{q}_{m}$ appears in the synchronization error dynamics (3.22 - 3.24) as a non-vanishing disturbance. Therefore the origin of the closed loop error space is not an equilibrium any more for (3.22 - 3.24). The best that can be expected is that the closed loop errors will be ultimately bounded, with a ultimate bound function of the master acceleration $\ddot{q}_{m}$.

\subsubsection{Stability analysis}

The following assumption is required in order to prove the convergence properties of the synchronization closed loop system. 
Assumption 3.11 The signals $\dot{q}_{m}(t)$ and $\ddot{q}_{m}(t)$ are bounded, i.e. there exist $V_{M}$ and $A_{M}$ such that

$$
\begin{aligned}
& \sup _{t}\left\|\dot{q}_{m}(t)\right\|=V_{M}<\infty \\
& \sup _{t}\left\|\ddot{q}_{m}(t)\right\|=A_{M}<\infty
\end{aligned}
$$

In practice, it is often not difficult to obtain bounds for $\dot{q}_{m}(t)$ and $\ddot{q}_{m}(t)$ based on the desired motion $q_{d}(t), \dot{q}_{d}(t)$ and $\ddot{q}_{d}(t)$ of the master robot. Although due to friction effects, tracking errors, etc., the actual motion of the master robot may differ from its desired motion, one could still deduce suitable values for $V_{M}$ and $A_{M}$. Also the bounds $V_{M}$ and $A_{M}$ can be obtained by considering the structural limitations of the robots, such as maximum velocities and accelerations of the motors.

The stability properties of the synchronization closed loop system are summarized in the following theorem.

Theorem 3.12 Consider the master and slave robots described by (3.1), the slave robot in closed loop with the control law (3.9), and both observers (3.10), (3.12). Introduce scalar parameters $\varepsilon_{o}, \lambda_{o}, \mu_{o}, \gamma_{o}$, defined throughout the proof, such that

$$
\lambda_{o}>0, \quad \mu_{o}>0, \quad \gamma_{o}>0, \quad \varepsilon_{o}>0
$$

Then the synchronization closed loop errors $\dot{e}, e, \widetilde{\dot{e}}, \widetilde{e}, \widetilde{\dot{q}}_{s}, \widetilde{q}_{s}$ are semi-globally uniformly ultimately bounded if the gain matrices $K_{d}, K_{p}, L_{p 1}, L_{p 2}$ are chosen such that their minimum eigenvalues, denoted by $K_{d, m}, K_{p, m}, L_{p 1, m}$, and $L_{p 2, m}$, satisfy

$$
\begin{aligned}
& L_{p 2, m}>\max \left\{\mu_{o}^{2}, \gamma_{o}^{2}, L_{p 2 q 4}, L_{p 2 q 5}, L_{p 2 q 6}\right\} \\
& L_{p 1, m}>\max \left\{2 \mu_{o}, L_{p 1 q 3}, L_{p 1 q 5, a}, L_{p 1 q 5, b}\right\} \\
& K_{p, m}>\max \left\{K_{p q 2}, K_{p q 6}\right\} \\
& K_{d, m}>\max \left\{K_{d q 1}\right\}
\end{aligned}
$$

Moreover, the ultimate bound can be made arbitrarily small, by a proper choice of $K_{p, m}$ and $L_{p 1, m}$.

The scalars $L_{p 2 q 4}, L_{p 2 q 5}, L_{p 2 q 6}, L_{p 1 q 3}, L_{p 1 q 5, a}, L_{p 1 q 5, b}, K_{p q 2}, K_{p q 6}, K_{d q 1}$ are defined in the gain tuning procedure given in Section 3.4.

Proof: The proof is based on a Lyapunov function, whose time derivative along the synchronization error dynamics $(3.22-3.24)$ is negative in an annulus around the origin. In this section a sketch of the proof is given. The details behind the sketch of the proof can be found in Appendix B.

Remark 3.13 Note that the conditions implied by Theorem 3.12 and the Assumptions 3.5, 3.6, 3.11 are only sufficient, but not necessary, to ensure stability and boundedness of the synchronization system. Hence for different values of $\lambda_{0}, \varepsilon_{0}, \mu_{0}$ and $\gamma_{0}$, different minimum eigenvalues of the gains $K_{p}, K_{d}, L_{p 1}$ and $L_{p 2}$ would be obtained. 


\section{Lyapunov function}

Consider the vector $y \in \mathbb{R}^{6 n}$ defined by (3.19), and take as a candidate Lyapunov function

$$
V(y)=\frac{1}{2} y^{T} P(y) y
$$

where $P(y)=P(y)^{T}$ is given by

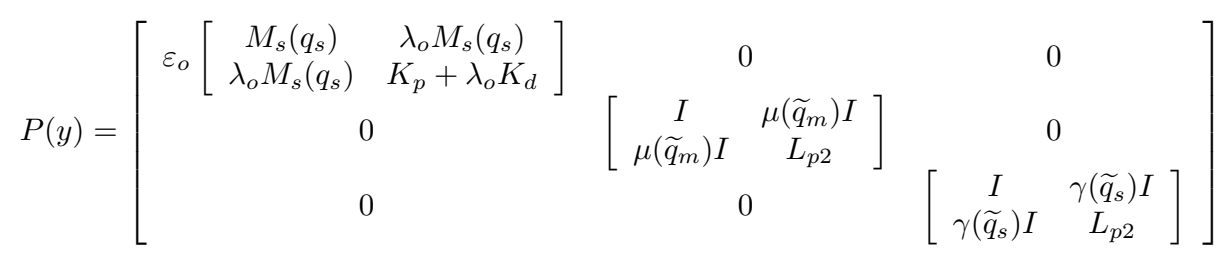

$\varepsilon_{o}, \lambda_{o} \in \mathbb{R}$ are positive constants to be determined, and $\mu\left(\widetilde{q}_{m}\right), \gamma\left(\widetilde{q}_{s}\right)$ are defined by

$$
\mu\left(\widetilde{q}_{m}\right):=\frac{\mu_{o}}{1+\left\|\widetilde{q}_{m}\right\|}, \quad \gamma\left(\widetilde{q}_{s}\right):=\frac{\gamma_{o}}{1+\left\|\widetilde{q}_{s}\right\|}
$$

with $\mu_{o}, \gamma_{o} \in \mathbb{R}$ positive constants to be determined; $\mu\left(\widetilde{q}_{m}\right), \gamma\left(\widetilde{q}_{s}\right)$ are bounded, such that

$$
0<\mu\left(\widetilde{q}_{m}\right) \leq \mu_{o}, \quad \text { and } \quad 0<\gamma\left(\widetilde{q}_{s}\right) \leq \gamma_{o}
$$

Sufficient conditions for positive definiteness of $P(y)$ are

$$
K_{d, m}>\lambda_{o} M_{s, M}, \quad L_{p 2, m}>\max \left\{\mu_{o}^{2}, \gamma_{o}^{2}\right\}
$$

Therefore, conditions $(3.27,3.28)$, together with the boundedness of $\mu\left(\widetilde{q}_{m}\right), \quad \gamma\left(\widetilde{q}_{s}\right)$, imply that there exist constants $P_{m}$ and $P_{M}$ such that

$$
\frac{1}{2} P_{m}\|y\|^{2} \leq V(y) \leq \frac{1}{2} P_{M}\|y\|^{2}
$$

\section{Derivative of the Lyapunov function and negative definiteness of $\dot{V}(y)$}

Along the error dynamics (3.22 - 3.24), by using Assumption 3.6, and by introducing the vector $y_{N}$ as

$$
y_{N}^{T}:=\left[\begin{array}{llllll}
\| \dot{\bar{q}} & \|\bar{q}\| & \left\|\dot{\widetilde{q}}_{m}\right\| & \left\|\widetilde{q}_{m}\right\| & \left\|\dot{\widetilde{q}}_{s}\right\| & \left\|\widetilde{q}_{s}\right\|
\end{array}\right]
$$

it follows that the time derivative of (3.29), i.e. $\dot{V}(y)$, has an upperbound given by, (see Appendix B for a detailed computation)

$$
\dot{V}(y) \leq\left\|y_{N}\right\|\left(\alpha_{0}-Q_{N m}\left\|y_{N}\right\|+\alpha_{2}\left\|y_{N}\right\|^{2}\right)
$$

where $Q_{N m}>0$ is the minimum eigenvalue of the matrix $Q_{N}=Q_{N}^{T}$

$$
Q_{N}=\left[\begin{array}{lll}
Q_{11_{N}} & Q_{12_{N}} & Q_{13_{N}} \\
Q_{12_{N}}^{T} & Q_{22_{N}} & Q_{23_{N}} \\
Q_{13_{N}}^{T} & Q_{23_{N}}^{T} & Q_{33_{N}}
\end{array}\right]
$$


with the block matrices

$$
\begin{aligned}
& Q_{11_{N}}=\varepsilon_{o}\left[\begin{array}{cc}
K_{d, m}-\lambda_{o} M_{s, M} & \frac{1}{2} \lambda_{o} V_{M}\left(C_{s, M}-M_{s, p M}\right) \\
\frac{1}{2} \lambda_{o} V_{M}\left(C_{s, M}-M_{s, p M}\right) & \lambda_{o} K_{p, m}
\end{array}\right] \\
& Q_{12_{N}}=\frac{\varepsilon_{o}}{2}\left[\begin{array}{cc}
-M_{s, M} L_{p 1, M} & K_{p, M}-K_{d, M} L_{p 1, M}-C_{s, M} L_{p 1, M} V_{M} \\
-\lambda_{o} M_{s, M} L_{p 1, M} & \lambda_{o}\left(K_{p, M}-K_{d, M} L_{p 1, M}-C_{s, M} L_{p 1, M} V_{M}\right)
\end{array}\right] \\
& Q_{13_{N}}=\frac{\varepsilon_{o}}{2}\left[\begin{array}{cc}
-K_{d, M} & -K_{d, M} L_{p 1, M} \\
-\lambda_{o} K_{d, M} & -\lambda_{o} K_{d, M} L_{p 1, M}
\end{array}\right] \\
& Q_{22_{N}}=\left[\begin{array}{cc}
L_{p 1, m}-2 \mu_{o} & \frac{1}{2}\left(M_{s, m}^{-1} K_{p, M}+\mu_{o} L_{p 1, M}\right) \\
\frac{1}{2}\left(M_{s, m}^{-1} K_{p, M}+\mu_{o} L_{p 1, M}\right) & \mu_{o}\left(M_{s, m}^{-1} K_{p, m}+L_{p 2, m}\right)
\end{array}\right] \\
& Q_{23_{N}}=\left[\begin{array}{cc}
0 & \frac{1}{2} M_{s, m}^{-1} K_{M} \\
\frac{1}{2}\left(M_{s, m}^{-1} K_{p, M}+L_{p 2, M}\right) & \frac{1}{2}\left(\left(\mu_{o}+\gamma_{o}\right) M_{s, m}^{-1} K_{p, M}+\gamma_{o} L_{p 2, M}\right)
\end{array}\right] \\
& Q_{33_{N}}=\left[\begin{array}{cc}
L_{p 1, m}-2 \gamma_{o}+2 M_{s, m}^{-1} C_{s, M} V_{M} & q_{56} \\
q_{56} & q_{66}
\end{array}\right] \\
& q_{56}=\frac{1}{2}\left(M_{s, m}^{-1} K_{p, M}+\gamma_{o} L_{p 1, M}\right)+M_{s, m}^{-1} C_{s, M} V_{M}\left(L_{p 1, M}+\gamma_{o}\right) \\
& q_{66}=\gamma_{o}\left(M_{s, m}^{-1} K_{p, m}+L_{p 2, m}+2 M_{s, m}^{-1} C_{s, M} V_{M} L_{p 1, m}\right)
\end{aligned}
$$

and $\alpha_{0}, \alpha_{2}$ are positive scalars given by

$$
\begin{gathered}
\alpha_{0}=\left(1+\sqrt{\mu_{o}}\right) \sqrt{A_{M}} \\
\alpha_{2}=\sqrt{8 M_{s, m}^{-1} C_{s, M}}\left(\sqrt{\gamma_{o}}+\sqrt{L_{p 1, M}}\right) \\
+\sqrt{\varepsilon_{o} C_{s, M}}\left(1+\sqrt{\lambda_{o}}\right)\left(L_{p 1, M}+2 \sqrt{L_{p 1, M}}\right) \\
+M_{s, m}^{-1} C_{s, M}\left(5+\sqrt{\gamma_{o}+2 L_{p 1, M}}+\sqrt{\gamma_{o} L_{p 1, M}+L_{p 1, M}^{2}+\gamma_{o}}+L_{p 1, M} \sqrt{\gamma_{o}}+\right. \\
+\sqrt{8 \gamma_{o} L_{p 1, M}}+\varepsilon_{o}\left(\sqrt{C_{s, M}}\left(1+2 \sqrt{L_{p 1, M}}\right)+\sqrt{\lambda_{o}\left(M_{s, p M}+C_{s, M}\right)}\right)+ \\
+\sqrt{\varepsilon_{o} \lambda_{o}}\left(2 \sqrt{C_{s, M}}\left(1+\sqrt{L_{p 1, M}}\right)+\sqrt{M_{s, p M}+C_{s, M}}\right)
\end{gathered}
$$

If the gains $K_{d}, K_{p}, L_{p 1}, L_{p 2}$ and the constants $\varepsilon_{o}, \lambda_{o}, \mu_{o}, \gamma_{o}$ satisfy conditions (3.27) and (3.28), then $Q_{N}$ given by (3.36) is positive definite. Then the right-hand side in (3.35) corresponds to (2.17), and together with (3.33) and Proposition 2.23, this allows to conclude local uniform ultimate boundedness of $y_{N}(3.34)$ and consequently of $y$ (3.19). By (3.20) we therefore conclude that the original state $x$, given by (3.18), is locally uniformly ultimately bounded.

Moreover, $\alpha_{2}$ depends explicitly on $L_{p 1, M}$, so that $y_{2}$ defined as in Proposition 2.23 can be made arbitrarily small by a proper chose of $L_{p 1, M}$, and thus the ultimate bound for the closed loop errors $\dot{e}, e, \widetilde{\dot{e}}, \widetilde{e}, \widetilde{\dot{q}}_{s}, \widetilde{q}_{s}$ can be made arbitrarily small. Notice that the minimum value for $y_{2}$ is given by $Q_{N m} /\left(2 \alpha_{2}\right)$, and recall that $Q_{N}$ (3.36) depends on $K_{p, m}$. 
On the other hand, a region of attraction is given by

$$
B=\left\{x \in \mathbb{R}^{6 n} \quad \mid \quad\|x\|<\frac{y_{2}}{\|T\|} \sqrt{\frac{P_{m}}{P_{M}}}\right\}
$$

where $T$ is given by $(3.21), P_{m}, P_{M}$ are defined by (3.33), and $y_{2}$ as in Proposition 2.23 , with (2.17) given by (3.35). Since the size of the region of attraction $B(3.39)$ is proportional to $y_{2}$, this region can be expanded by increasing $y_{2}$. Thus the uniform ultimate bounded result is semi-global.

Remark 3.14 The conditions given by (3.28) imply relations between the minimum and maximum eigenvalues of $L_{p 1}, L_{p 2}$, and at the same time $Q_{N m}$ and $\alpha_{2}$ depend on the maximum eigenvalue of $L_{p 1}$. All these relations have to be taken into account to choose the control and observer gains $K_{d}, K_{m}, L_{p 1}, L_{p 2}$. Nevertheless $y_{2}>\delta y_{1}$ (see Proposition 2.23) can be always satisfied.

On the other hand $L_{p 2}$ is also related to the value of $P_{M}$, see (3.33), such that by increasing $L_{p 2}, P_{M}$ also increases. But there is still freedom on the gain $K_{d}$, such that the ratio $P_{m} / P_{M}$ can be kept far from zero, and thus shrinking of the region of attraction is avoided.

The ultimate boundedness result of the synchronization closed loop system is due to the absence of measurements of $\ddot{q}_{m}$, see (3.35) and (3.37), such that the ultimate bound of the errors is proportional to the master acceleration $\ddot{q}_{m}$. This yields the following Corollary in case of set point regulation of the master robot.

Corollary 3.15 If set point regulation of the master robot is considered and the master robot controller is able to achieved steady state in finite time, then $\dot{q}_{m}(t)=0$, $\ddot{q}_{m}(t)=0$ for $t \in\left(t_{2}, \infty\right), t_{2} \geq t_{0}$, being the convergence time of the master robot trajectories. If the conditions on Theorem 3.12 are satisfied, then the control law (3.9), and both observers (3.10), (3.12) yield semi-global exponential convergence of the synchronization closed loop errors $\dot{e}, e, \widetilde{\dot{e}}, \widetilde{e}, \widetilde{\dot{q}}_{s}, \widetilde{q}_{s}$ for $t \geq t_{2}$.

Proof: From (3.35), (3.37) we have the following. If the conditions in Theorem 3.12 are satisfied, and $\ddot{q}_{m}(t)=0$ for $t \in\left(t_{2}, \infty\right), t_{2} \geq t_{0}$, then for $t \geq t_{2}$ (3.35) reduces to

$$
\dot{V}(y) \leq\left\|y_{N}\right\|^{2}\left(-Q_{N m}+\alpha_{2}\left\|y_{N}\right\|\right)
$$

with $Q_{N m}>0$.

On the other hand, the region of attraction (3.39) guarantees that $Q_{N m}>\alpha_{2}\left\|y_{N}\right\|$, and thus $\dot{V}(y)$ has an upperbound given by

$$
\dot{V}(y) \leq-\kappa\left\|y_{N}\right\|^{2} \quad \text { for all } t \geq t_{2}
$$

From the last inequality and (3.33), we conclude that there exist some constants $m^{*}, \rho>0$, such that

$$
\|y(t)\|^{2} \leq m^{*} e^{-\rho t}\left\|y\left(t_{2}\right)\right\|^{2} \quad \text { for all } t \geq t_{2}
$$

by (3.20) we can conclude the same for $x$ given by (3.18). 
Remark 3.16 Throughout the presentation and analysis of the synchronization controller, it has been assumed that the master robot has a controller that assures convergence to a desired trajectory. The master controller is independent from the synchronization controller presented in Section 3.3. Therefore, the master robot convergence time and stability properties are irrelevant for the proposed synchronization controller. It can even happen that the master robot convergence time is $\infty$, like in case of asymptotic stability of the master robot.

\subsection{Gain tuning procedure}

The following step by step gain tuning procedure gives a constructive way to guarantee stability and ultimate boundedness of the synchronization closed loop system, such that synchronization between the master and slave robots is achieved.

1. Determine the bounds of the master trajectories $\dot{q}_{m}, \ddot{q}_{m}$, the physical parameters $M_{s}\left(q_{s}\right), C_{s}\left(q_{s}, \dot{q}_{s}\right)$ and their partial derivatives with respect to $q_{s}$.

2. Choose the weighting factors $\lambda_{0}>0, \varepsilon_{0}>0, \mu_{0}>0$ and $\gamma_{0}>0$ and a bound for the maximum eigenvalue of the gains $K_{p}, K_{d}, L_{p 1}$ and $L_{p 2}$.

3. By considering the matrix $Q_{N}$ given by (3.36) determine the minimum eigenvalue of the gain matrices $K_{p}, K_{d}, L_{p 1}$ and $L_{p 2}$, such that $Q_{N}$ is a positive definite matrix. For this a set of nonlinear algebraic equations must be solved.

Let $\Delta Q_{i}$ denote the determinant of the $i$-th leading minor of $Q_{N}$. Then sufficient conditions for positive definiteness of $Q_{N}$ are given by (3.28) with $L_{p 2 q 4}, L_{p 2 q 5}, L_{p 2 q 6}$, $L_{p 1 q 3}, L_{p 1 q 5, a}, L_{p 1 q 5, b}, K_{p q 2}, K_{p q 6}$, and $K_{d q 1}$ given by

- $K_{d q 1}=\lambda_{o} M_{s, M}$,

- $K_{p q 2}=\frac{\lambda_{o} V_{M}\left(M_{s, p M}-C_{s, M}\right)^{2}}{4\left(K_{d, m}-\lambda_{o} M_{s, M}\right)}$,

- $L_{p 1 q 3}$ : denotes the solution of the equation $\Delta Q_{3}=a_{1} L_{p 1 q 3}+a_{2}=0$, with $a_{1}, a_{2}$ the resultant coefficients in the factorization of $L_{p 1, m}$ in $\Delta Q_{3}$, and $L_{p 1, m}$ substituted by $L_{p 1 q 3}$.

- $L_{p 2 q 4}$ : denotes the solution of the equation $\Delta Q_{4}=b_{1} L_{p 2 q 4}+b_{2}=0$, with $b_{1}, b_{2}$ the resultant coefficients in the factorization of $L_{p 2, m}$ in $\Delta Q_{4}$, and $L_{p 2, m}$ substituted by $L_{p 2 q 4}$.

- $L_{p 1 q 5, a}=2\left(\gamma_{o}-C_{s, M} V_{M} M_{s, M}^{-1}\right)$,

- $L_{p 1 q 5, b}$ : denotes the largest solution of the equation $\Delta Q_{5}=c_{0}+c_{1} L_{p 1 q 5}+$ $c_{2} L_{p 1 q 5}^{2}=0$, with $c_{0}, c_{1}, c_{2}$ the resultant coefficients in the factorization of $L_{p 1, m}$ in $\Delta Q_{5}$, and $L_{p 1, m}$ substituted by $L_{p 1 q 5}$.

- $L_{p 2 q 5}$ : denotes the solution of the equation $c_{2}=r_{1} L_{p 2 q 5}+r_{2}=0$, with $c_{2}$ as in $L_{p 1 q 5, b}, r_{1}, r_{2}$ the resultant coefficients in the factorization of $L_{p 2, m}$ in $c_{2}$, and $L_{p 2, m}$ substituted by $L_{p 2 q 5}$. 
- $L_{p 2 q 6}:$ denotes the largest solution of the equation $\Delta Q_{6}=t_{o}+t_{1} L_{p 2 q 6}+$ $t_{2} L_{p 2 q 6}^{2}=0$, with $t_{0}, t_{1}, t_{2}$ the resultant coefficients in the factorization of $L_{p 2, m}$ in $\Delta Q_{6}$, and $L_{p 2, m}$ substituted by $L_{p 2 q 6}$.

- $K_{p q 6}$ : denotes the solution of the equation $t_{2}=s_{1} K_{p q 6}+s_{2}=0$, with $t_{2}$ as in $L_{p 2 q 6}, s_{1}, s_{2}$ the resultant coefficients in the factorization of $K_{p, m}$ in $t_{2}$, and $K_{p, m}$ substituted by $K_{p q 6}$.

\subsection{Friction compensation}

This section presents the extension of the synchronization scheme of Section 3.3 in case that robots with friction effects are considered. The synchronization scheme developed here has been implemented to obtain the experimental results reported in Section 3.6 and Chapter 6. A sketch of the stability proof of the synchronization closed loop system is given. A formal analysis goes along the same lines as given in the proof of Theorem 3.12.

The dynamic model (3.1) does not account for friction phenomena. Nonetheless friction phenomena always appear in practical implementations and have to be compensated in order to improve the performance of the robots. How to compensate for friction phenomena highly depends on the way of modelling such effects. Because of this, compensation of friction phenomena in the proposed synchronization schemes is treated and presented separately from the synchronization problem. The core of separate compensation of friction phenomena in robot manipulators lies in the fact that the effects of friction phenomena are in general bounded as function of the velocity, and moreover friction phenomena appear as an additive term in the dynamic model (3.1).

First we analyze the scenarios that are obtained for feedforward and feedback friction compensation in the synchronization controller proposed in Section 3.3. Recall that throughout this thesis the particular friction model given by (3.3) is considered.

In case of feedforward friction compensation there is the requirement of master robot velocity measurements $\dot{q}_{m}$ (as desired or commanded synchronization trajectory), see the synchronization feedback control (3.9) and the synchronization errors (3.7). However, it has been assumed that such measurements are not available, and neither is the master robot dynamic model, so that model based observers can also not be designed. Therefore, if feedforward friction compensation is considered the master robot velocity $\dot{q}_{m}$ has to be reconstructed by using non-model based observers, e.g. high gain, or numerical differentiation and filtering techniques, with implicit implementation and bandwidth problems, see Section 1.3.1. On the other hand the observer proposed in Section 3.3.3 reconstructs the instantaneous slave robot velocity $\dot{q}_{s}$ that can be used directly for feedback friction compensation. Therefore, for friction compensation in the synchronization controller presented in Section 3.3, feedback techniques are chosen over feedforward techniques.

Considering the friction model given by (3.3) and the dynamic model of the robot manipulators given by (3.2), it follows that the feedback controller $\tau_{s}(3.9)$ can be modified as

$$
\begin{aligned}
\tau_{s f} & =\tau_{s}+f_{s}\left(\widehat{\dot{q}}_{s}\right) \\
& =M_{s}\left(q_{s}\right) \widehat{\dot{q}}_{m}+C_{s}\left(q_{s}, \widehat{\dot{q}}_{s}\right) \widehat{\dot{q}}_{m}+g_{s}\left(q_{s}\right)-K_{d} \widehat{\dot{e}}-K_{p} e+f_{s}\left(\hat{\dot{q}}_{s}\right)
\end{aligned}
$$


with the friction compensation term $f_{s}\left(\widehat{\dot{q}}_{s}\right)$ given by

$$
f_{s}\left(\widehat{\dot{q}}_{s}\right)=B_{v, s} \widehat{\dot{q}}_{s}+B_{f 1, s}\left(1-\frac{2}{1+e^{2 w_{1, s} \hat{q}_{s}}}\right)+B_{f 2, s}\left(1-\frac{2}{1+e^{2 w_{2, s} \hat{\dot{q}}_{s}}}\right)
$$

and $\widehat{\dot{q}}_{s}$ the estimated slave velocity obtained by the observer (3.12).

The estimates for the velocity synchronization error $\widehat{\dot{e}}$ and the master velocity and acceleration $\widehat{\dot{q}}_{m}, \widehat{\ddot{q}}_{m}$ are obtained by the same observers as in the frictionless case, i.e. observer (3.10) and relations (3.14).

The stability analysis of the closed loop system formed by the synchronization controller (3.40), the observers (3.10), (3.12) and the slave robot goes in the same way as for frictionless rigid join robots. The support of the stability analysis is the fact that the friction model (3.3) implies that

$$
\left\|f_{s}\left(\widehat{\dot{q}}_{s}\right)-f_{s}\left(\dot{q}_{s}\right)\right\| \leq B_{v, s M}\left\|\widetilde{\dot{q}}_{s}\right\|+2 B_{f 1, s M}+2 B_{f 2, s M}
$$

with $\widetilde{\dot{q}}_{s}$ the velocity estimation error, and $B_{v, s M}, B_{f 1, s M}, B_{f 2, s M}$ the maximum eigenvalue of the coefficient matrices $B_{v, s}, B_{f 1, s}, B_{f 2, s}$.

Because the friction effects appear as an additive term in the robot dynamics (3.1) and the controller (3.40), then the difference $f_{s}\left(\hat{\dot{q}}_{s}\right)-f_{s}\left(\dot{q}_{s}\right)$ appears in the synchronization closed loop error. Then by considering the Lyapunov function (3.29) it follows that the bound (3.41) appears in the bound of the derivative of the Lyapunov function (3.35). Following the same steps as in the frictionless rigid robot case it can be concluded that semi-global uniform stability is obtained.

\subsection{Simulation and experimental study}

The proposed synchronization controller for rigid joint robots $\tau_{s f}$, given by (3.40), has been implemented on a one degree of freedom master and slave system. The master and slave system correspond to the medium link of two identical transposer robots fabricated by the Centre for Manufacturing Technology (CFT) Philips Laboratory. The experimental setup, the dynamic model and the estimated parameters for the robots are given in Section 2.3. The master robot corresponds to robot 1 in Section 2.3 , while robot 2 has the role of slave robot. The desired trajectory for the master robot $q_{d}(t)$ is a harmonic series given by $(2.35)$.

The master and slave controllers $\tau_{m f}, \tau_{s f}$ are implemented according to (3.40), with $\tau_{m}, \tau_{s}$ as in (3.9). The friction compensation term $f_{i}\left(\widehat{\dot{q}}_{i}\right)$ is a function of the estimated velocity $\widehat{\dot{q}}_{i}$, that is obtained by the observer (3.12).

From the tuning gain procedure given on Section 3.4, it follows that for the values $\lambda_{0}=5 \times 10^{-7}, \varepsilon_{0}=0.0003, \mu_{0}=0.0001, \gamma_{0}=0.02$, the synchronization system is stable and the errors are bounded if the slave scalar gains satisfy $K_{p, s}>0, K_{d, s}>$ $8 \times 10^{-7}, L_{p 1, s}>35$, and $L_{p 2, s}>0$. Since the physical parameters of the master and slave robot are very similar, it is expected that similar values of the gains would render a stable master system. After a series of experiments in order to decrease the synchronization position error $e$, the gains on the master and slave controllers were set as listed in Table 3.1. These gains have been used in all the experiments and simulations presented in this chapter. 


\begin{tabular}{|c|c|c|c|c|}
\hline & $K_{p, i}$ & $K_{d, i}$ & $L_{p 1, i}$ & $L_{p 2, i}$ \\
\hline master robot & 2500 & 80 & 100 & 2200 \\
\hline slave robot & 2500 & 80 & 100 & 2200 \\
\hline
\end{tabular}

Table 3.1: Control gains in master and slave controllers.

\begin{tabular}{|c|c|c|c|}
\hline & $q_{i}(0)[\mathrm{rad}]$ & $\dot{q}_{i}(0)[\mathrm{rad} / \mathrm{s}]$ & $\widehat{q}_{i}(0)[\mathrm{rad}]$ \\
\hline master robot & 2.13 & 0 & 1.98 \\
\hline slave robot & 2.00 & 0 & 1.98 \\
\hline & $\hat{q}_{i}(0)[\mathrm{rad} / \mathrm{s}]$ & $\widehat{e}_{i}(0)[\mathrm{rad}]$ & $\dot{e}_{i}(0)[\mathrm{rad} / \mathrm{s}]$ \\
\hline master robot & 0 & 0.1 & 0 \\
\hline slave robot & 0 & 0.1 & 0 \\
\hline
\end{tabular}

Table 3.2: Initial conditions for master and slave robot.

The initial position of the links and the initial conditions in the observers (3.10), (3.12) were chosen as in Table 3.2

First a comparative study between simulated and experimental results is presented. The experimental results closely match the simulated results, which validates the model of the system $(2.33,2.34)$ and the estimated physical parameters listed in table 2.1. Since the experimental results are so close to the simulated ones, the predicted stability and convergence properties of the proposed synchronization controller (3.9 or 3.40) are shown by experimental results rather than by numerical simulations.

\section{Simulation and experimental results}

The master and slave dynamic model $(2.33,2.34)$ were implemented in the standard Simulink distribution for Matlab version 6.0 Release 12 to numerically simulate the synchronization closed loop system. The gains and initial conditions were set as in Tables 3.1 and 3.2. The frequency of the master robot desired trajectory $q_{d}(t)$, given by (2.35), was set as $\omega=0.4 \mathrm{~Hz}$.

Figure 3.3 show the master and slave position trajectories, simulated $q_{m, s}, q_{s, s}$ and measured $q_{m}, q_{s}$. The simulated and measured positions match very well after the transient period has finished. The initial mismatch is due to large values of the input torques during the simulations, which exceed the maximum torque of the physical robots $\left(\tau_{\max }=500[\mathrm{Nm}]\right)$, see Figure 3.4. Boundedness of the input controls is an important open problem in Control Theory, since it can generate instability of the closed loop system. However the study of this problem is beyond the scope of this thesis.

The simulated and measured slave input torques $\tau_{s, s}$, and $\tau_{s}$ are presented in Figure 3.4. Note that $\tau_{s, s}$, and $\tau_{s}$ only correspond to the synchronization controller (3.9), i.e. they do not include the friction compensation term $f_{s}\left(\widehat{\dot{q}}_{s}\right)$ of the controller (3.40), although the controller (3.40) has been implemented in both the master and slave robot. Since there is no bound on the input control during the simulations, $\tau_{s, s}$ presents large overshoots during the transient. These overshoots are the reason of mismatch between the simulated and measured data during the transient period. After the transient has finished, the simulated and measured slave torques are quite similar. 

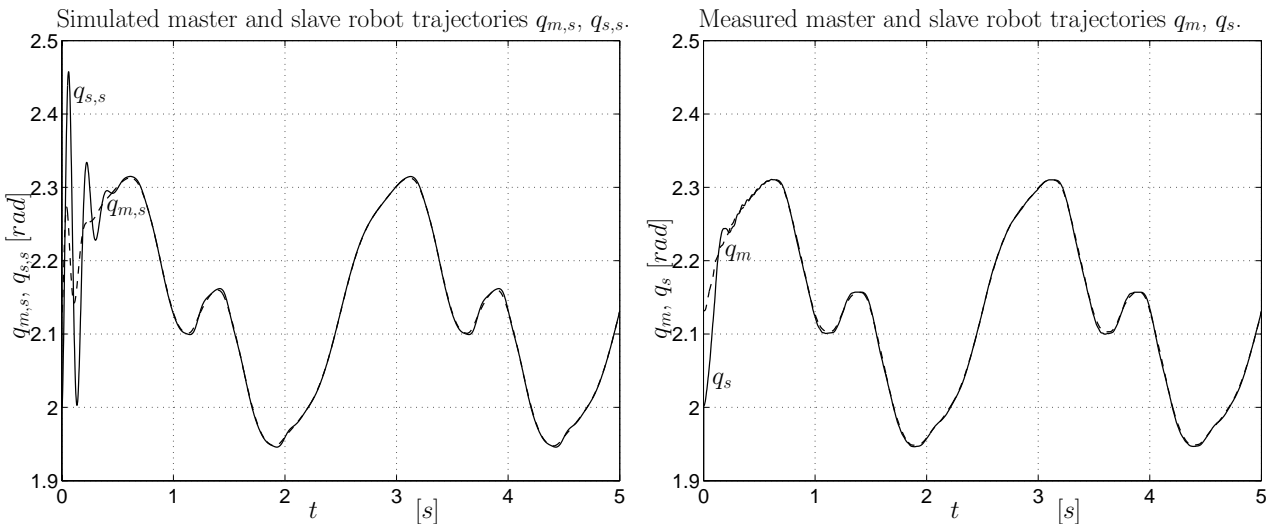

Figure 3.3: Master $q_{m}$ (dashed) and slave $q_{s}$ (solid) positions, simulated $\left(*_{, s}\right)$.
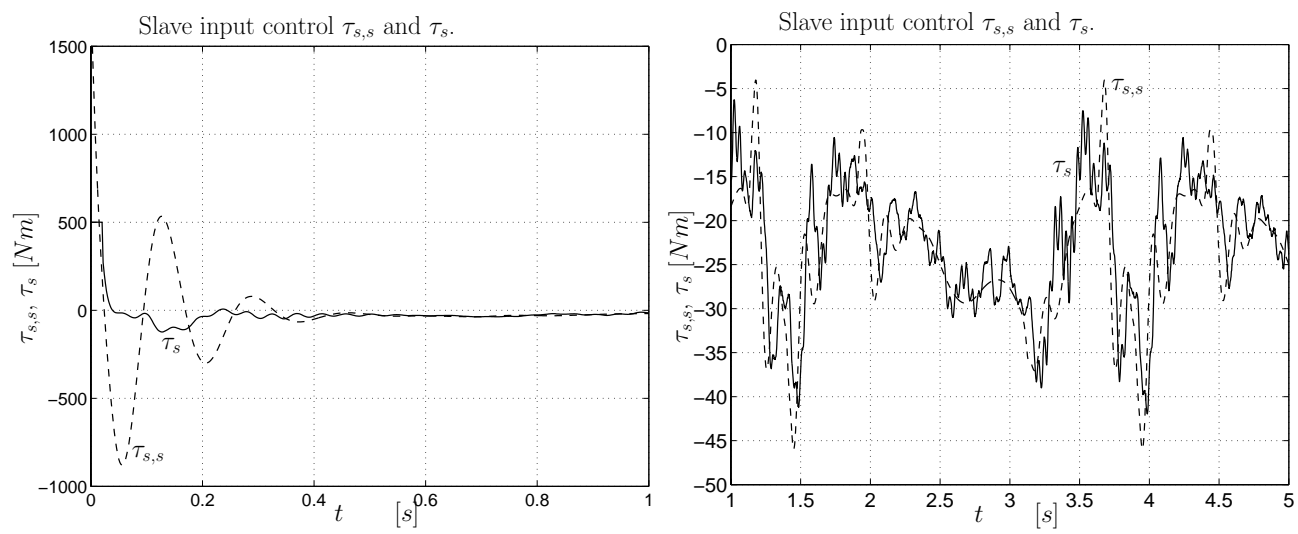

Figure 3.4: Slave input torques, simulated $\tau_{s, s}$ (dashed) and measured $\tau_{s}$ (solid).

The synchronization errors $e=q_{s}-q_{m}$, simulated and measured, are shown in Figure 3.5. After the transient period has finished the simulated and measured synchronization errors match very well.

Figure 3.6 presents the simulated $\left(*_{, s}\right)$ and measured estimated error for the synchronization error $\widetilde{e}$ (observer (3.10)) and for the joint slave position $\widetilde{q}_{s}$ (observer (3.12)) after the transient has finished. Both observers show good performance and convergence properties, and the simulated and real errors match almost perfectly.

\section{Comparative results for different controllers}

For the sake of comparison a PD and PID controller were also implemented on the slave system. The gains of the PD, PID controllers were set equal to the proportional and derivative gains in the synchronization controller, i.e. $K_{p}=2500, K_{d}=80$. The gain in the integral part of the PID control was set as $K_{i}=20$. The velocity in the friction compensation term $f_{s}\left(\widehat{\dot{q}}_{s}\right)$ was obtained by using the numerical differentiator function supplied in the DS1005 dSPACE system. The frequency of the master robot desired trajectory $q_{m, d}(t)$ was set as $\omega=0.2 \mathrm{~Hz}$, since it was observed that for frequencies higher than $\omega=0.2 \mathrm{~Hz}$ the closed loop system with the PD and PID controllers 

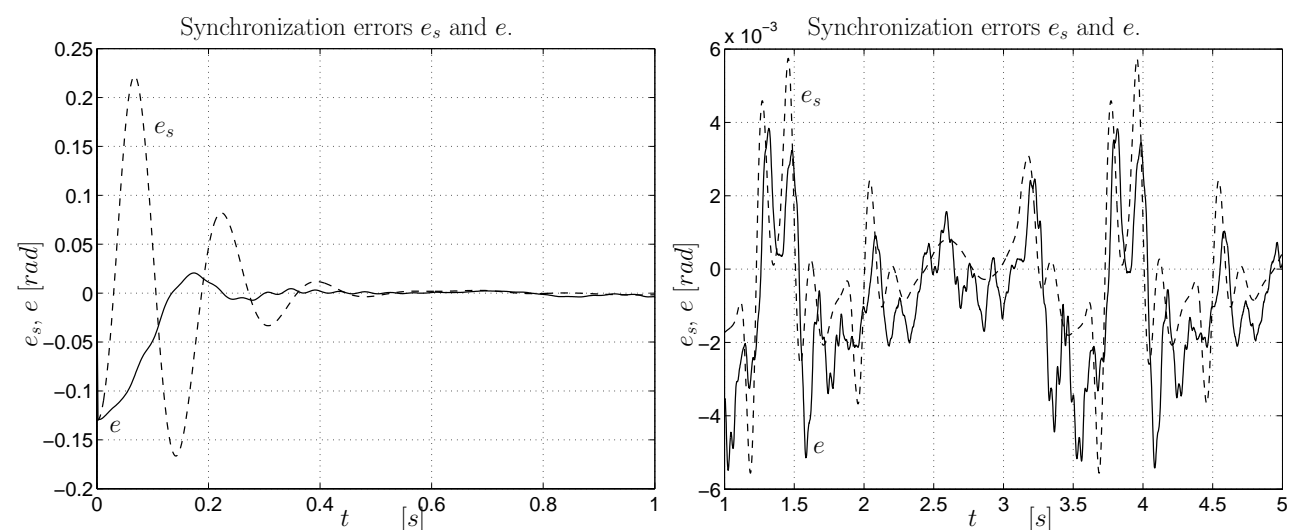

Figure 3.5: Synchronization errors, simulated $e_{s}$ (dashed) and measured $e$ (solid).
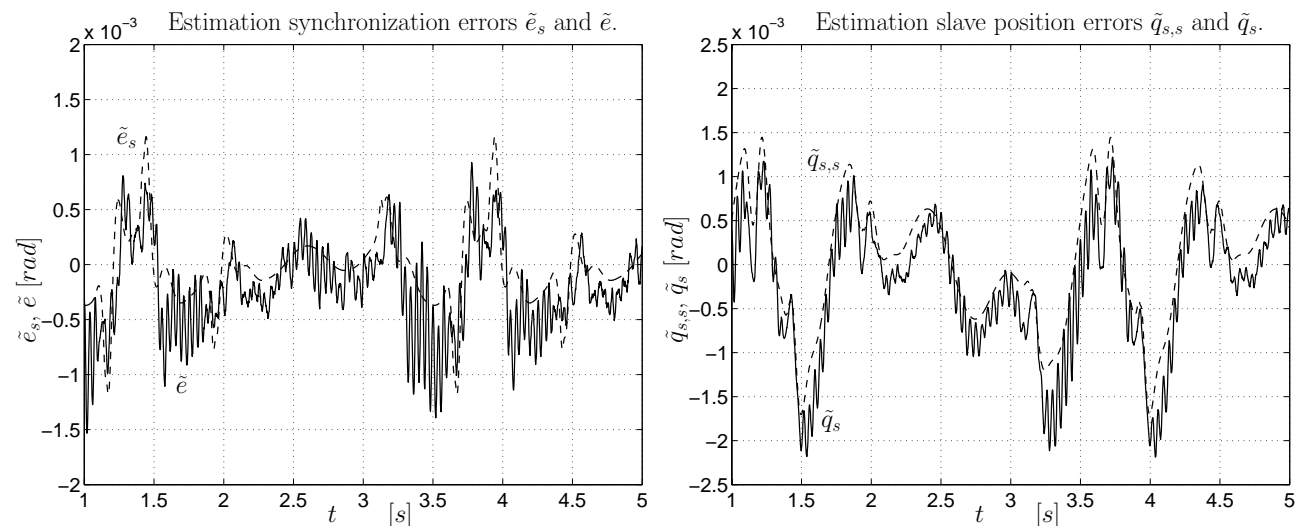

Figure 3.6: Estimation synchronization errors for observers (3.10) and (3.12), simulated $\widetilde{e}_{s}$ and $\widetilde{q}_{s, s}$ (dashed), measured $\widetilde{e}$ and $\widetilde{q}_{s}$ (solid).

became unstable. Note that the PD and PID controllers have not been optimized in any sense, but for comparison purposes their gains have been chosen equal to those of the synchronization controller. The values of the gains $K_{p}=2500, K_{d}=80$ in the synchronization controller have not been optimized either, but chosen by trial and error in between the limits obtained from the gain tuning procedure, Section 3.4.

Figure 3.7(left) presents the master $q_{m}$, and slave robot trajectories $q_{s}$ for the synchronization controller (3.9 or 3.40), the PD controller, $q_{s, P D}$, and the PID controller, $q_{s, P I D}$. Since the proportional $K_{p}$ and derivative $K_{d}$ gains are the same for all controllers, they showed similar performance during the transient, such as oscillations on the overshoot. However, after the transient has finished, the PD and PID showed poor convergence, while the synchronization controller $(3.9,3.40)$ showed good convergence between the master and slave trajectories, as can be seen form the synchronization errors shown in Figure 3.7(right). 

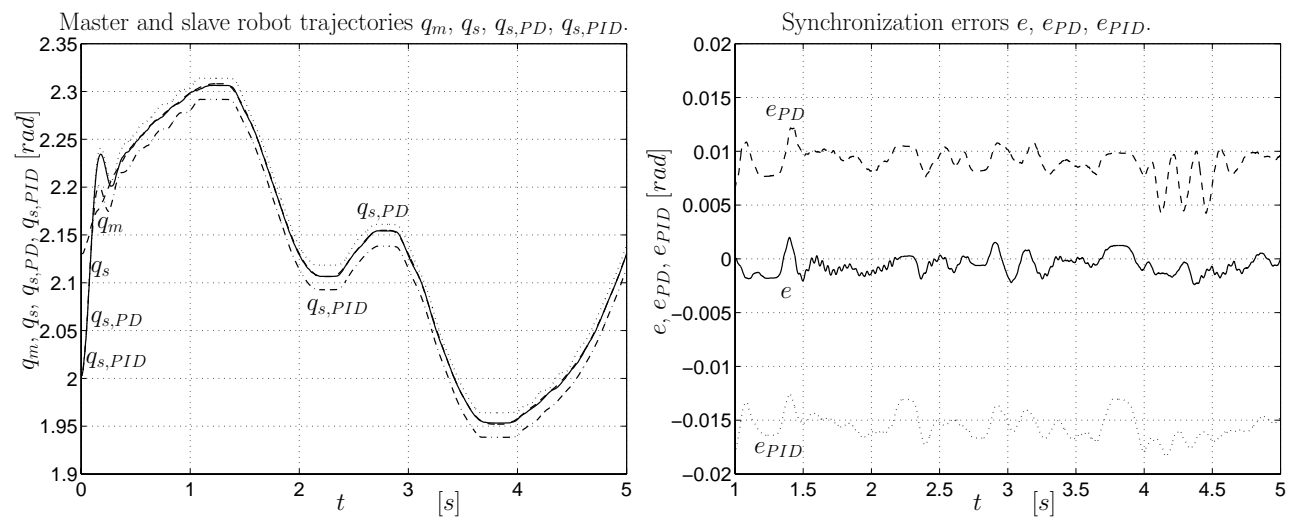

Figure 3.7: Master $q_{m}$ and slave positions $q_{s}$, and synchronization errors $e_{s}$ for controller (3.9) (solid), PD (dashed), and PID (dotted).

\section{Sensitivity to desired trajectories}

As mentioned in the Remark 3.10 the value of the ultimate bound is a function of the master acceleration $\ddot{q}_{m}$. To show this property, two different values for the frequency on the master desired trajectory $q_{m, d}(t)$ have been considered, $\omega=0.2,0.6 \mathrm{~Hz}$. None of the controller gains have been changed during the experiments.

Figure 3.8 show the master $q_{m}$ and slave $q_{s}$ robot position for $\omega=0.2,0.6 \mathrm{~Hz}$. Smaller synchronization errors are obtained when $\omega=0.2 \mathrm{~Hz}$ which agrees with the predicted performance of the synchronization controller.
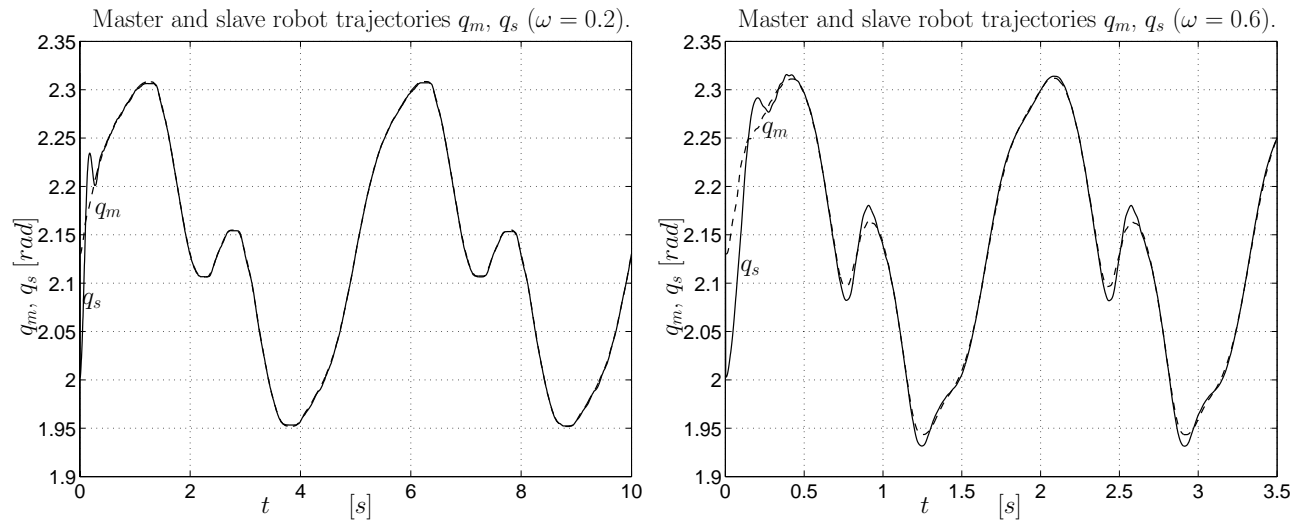

Figure 3.8: Master $q_{m}$ (dashed) and slave positions $q_{s}$ (solid) for $\omega=0.2, \omega=0.6$.

It is worth to mention that the estimated parameters listed in Section 2.3 were obtained based on a trajectory with frequency of $\omega=0.4 \mathrm{~Hz}$. Therefore the bandwidth of the dynamic model $(2.33,2.34)$ together with the estimated parameters listed in Table 2.1 is centered in $0.4 \mathrm{~Hz}$. Thus the synchronization errors for $\omega=0.2,0.6 \mathrm{~Hz}$ are also partially due to the estimated parameters. 


\section{Disturbance rejection}

The proposed synchronization controller is robust against disturbances and parametric uncertainty. A payload of $2.0 \mathrm{~kg}$. was used as a disturbance on the master and slave systems. The frequency on the desired trajectory $q_{m, d}(t)$ was set as $\omega=0.4 \mathrm{~Hz}$. Figure 3.9(left) shows the tracking error in the master robot $e_{m}=q_{m}-q_{m, d}$ and the synchronization error in the slave robot $e_{s}=q_{s}-q_{m}$ when the payload of $2.0 \mathrm{~kg}$ was set on the master robot, from $t=30.8 \mathrm{~s}$ to $t=39.3 \mathrm{~s}$. It can be observed that the load generates a jump in the master tracking error, while the slave robot is practically not affected. Figure 3.9(right) present the synchronization errors when the payload of $2.0 \mathrm{~kg}$ was set on the slave robot, from $t=104.5 \mathrm{~s}$ to $t=113.5 \mathrm{~s}$. As expected the master robot is not affected because there is not interaction from the slave to the master robot. On the other hand, it can be observed that the payload has minimum effects on the slave robot.
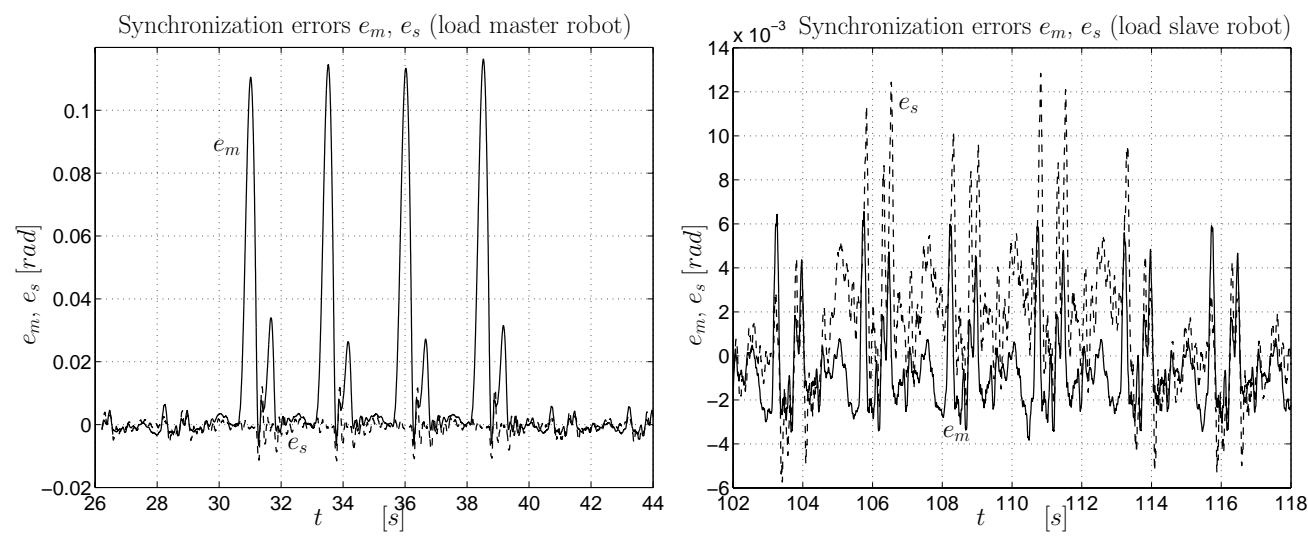

Figure 3.9: Payload of $2.0 \mathrm{~kg}$ in master and slave robot, $e_{m}$ (solid), $e_{s}$ (dashed).

The same controller $(3.9,3.40)$, with the same gains, has been implemented in both robots, master and slave. However the master robot seems to be more sensitive to disturbances than the slave robot. This sensitivity to disturbances that is shown by the master robot can be due to the differences in the physical parameters of the robots, particularly the friction coefficients. It can also be due to the accuracy of the estimated parameters, see the validation figure presented in Section 2.3. From Figure 2.3 it is evident that the slave robot parameters are better estimated than those of the master robot.

\subsection{Concluding remarks and discussion}

- The proposed synchronization controller yields practical position and velocity synchronization in the joint space. Position and velocity synchronization in the Cartesian space, i.e. spatial coordinates, is obtained only if the length of the links of the slave robot are equal to the corresponding links in the master robot.

- The variables $\widetilde{q}_{m}, \dot{\widetilde{q}}_{m}$, defined by (3.16), can be interpreted as the estimation error in the joint variables of the master robot $q_{m}, \dot{q}_{m}$. Therefore, $\widetilde{q}_{m}, \dot{\widetilde{q}}_{m}$ give an idea of how well the estimation of the master robot variables can be made 
based on measured and estimated variables of the slave robot. So, the slave robot, under the proposed synchronization controller, can be considered as a physical estimator for the master robot dynamics in the joint space.

- The uniform ultimate boundedness result is of semi-global nature, with region of attraction (3.39). This region of attraction and the bound for the closed loop errors depend on $y_{2}$ in a proportional way. This is an intrinsic property of the considered method, see proposition 2.23 and Section 3.3.6, and thus a compromise has to be made.

Nevertheless, the region of attraction mainly depends on the initial estimate errors. Therefore, if small initial estimate errors (see observers (3.10) and (3.12)) are chosen, then high initial synchronization errors can be considered. The high dependency on the estimate errors is the price to be paid for the lack of available measurements or high quality measurements.

- Even without knowledge of the bounds in (3.27) and (3.28), the synchronization closed loop system can be made uniformly ultimately bounded by selecting the control gains large enough. However, such high gain implementations are not always desirable in practical circumstances, since high gains might generate large overshoots during transients and amplify noisy measurements.

- The controller and observer gains in the proposed synchronization controller can be interpreted physically as damping or stiffness in the closed loop system. Consequently, an insight of how the gains affect the closed loop performance and how to tune them can be obtained, which in general is more difficult to determine for other techniques like variable structure implementations, sliding controls, etc..

- The controller and observers (3.9), (3.10) and (3.12) are model based, nevertheless the stability analysis allows a straightforward robustness analysis for parametric uncertainties. Because of linearity of the robot dynamical model (3.1), we have that parametric uncertainties appear as an additive term in $\dot{V}$, given by (B.1). Thus, if we consider bounded parametric uncertainties, then this new bounded term appears in (3.35). So, by retuning the gains we can ensure that $\dot{V}$ is negative definite, such that the convergence properties of the closed loop system are preserved.

In case of unmodelled dynamics the stability analysis is not valid any more in general, since it greatly depends in the type of unmodelled phenomena.

Adaptive extensions of the proposed synchronization scheme can be obtained by following the work presented in (Berghuis 1993), see for instance the work presented in (Lee and Chung 1998). Of course the dynamics of the adaptive part and the adaptation error dynamics must be considered in the stability analysis.

- The proposed synchronization controller (3.9), (3.10) and (3.12) provides a systematic way of proving stability and boundedness of the closed loop system. This is a drawback of some other schemes for estimating velocities, such as numerical differentiation or low pass filters. For those techniques, in general, there do not exist formal stability proofs or a methodology to guarantee stability of the synchronization closed loop system. 


\section{Chapter 4}

\section{External synchronization of flexible joint robots}

\subsection{Introduction}

This chapter addresses the problem of synchronization of robot manipulators with flexible joints. It is assumed that not all the joint state variables are measured, e.g. link and rotor velocities are unknown, which is a very common situation when joint flexibility is present. The main goal is to ensure synchronization between two (or more) robots, where the robots for which the control will be designed (slaves) have flexible joints, and the robot, whose trajectories are to be followed (master), may or may not have flexible joints. Results in this chapter have been reported on the articles (Rodriguez-Angeles and Nijmeijer 2002b), (Rodriguez-Angeles et al. 2002c).

Joint flexibility can be caused by transmission elements such as harmonic drives, belts, or long shafts, and considerably affects the performance of robot manipulators (Good et al. 1985), since it is a major source of oscillatory behavior. Joint flexibility can be modelled by considering the position and velocity of the motor rotor $\theta, \dot{\theta}$, and the position and velocity of the link $q, \dot{q}$. Therefore, the order of the dynamic model for a flexible joint is twice that of a rigid joint, consequently, the control laws proposed for flexible joint robots are more complex than those for rigid robots.

In this chapter we consider a multi-robot system formed by two fully actuated robots with $n$ joints each and working in a master-slave scheme, such that the subindices $m, s$ identify the master and slave robot respectively. Assume that the master robot is driven by an input torque $\tau_{m}(\cdot)$, which in the ideal case ensures convergence of the link position and velocity variables $q_{m}, \dot{q}_{m}$ to a desired trajectory $q_{d}, \dot{q}_{d}$. However, the input torque $\tau_{m}$, the dynamic model and parameters of the master robot, as well as the link velocity and acceleration variables $\dot{q}_{m}, \ddot{q}_{m}$, are not available for design of the slave control law $\tau_{s}(\cdot)$. Also we assume that the rotor position $\theta_{s}$, and the link and rotor velocities and accelerations $\dot{q}_{s}, \ddot{q}_{s}, \dot{\theta}_{s}, \ddot{\theta}_{s}$ are not available. Therefore, the control law $\tau_{s}$, which is to be designed such that the link variables $q_{s}, \dot{q}_{s} \in \mathbb{R}^{n}$ synchronize with the variables $q_{m}, \dot{q}_{m}$, can only depend on link position measurements of both robots, i.e. $q_{m}, q_{s}$, and estimated values of $\theta_{s}, \dot{\theta}_{s}, \dot{q}_{m}, \ddot{q}_{m}, \dot{q}_{s}, \ddot{q}_{s}$.

Figure 4.1 shows a schematic representation of the external synchronization problem for the flexible joint robots. 


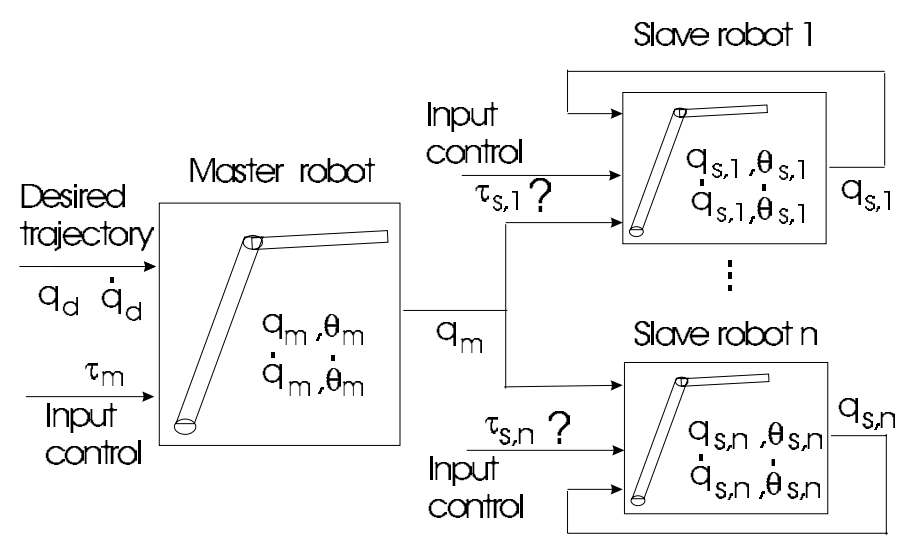

Figure 4.1: External synchronization of flexible joint robots.

Notice that the goal is to follow the link trajectories of the master robot $q_{m}, \dot{q}_{m}$, not the desired trajectories $q_{d}, \dot{q}_{d}$ for the master robot, which may not be realized due to model uncertainties or disturbances in the system, e.g. noise, unknown loads. Therefore, flexible joint robot synchronization occurs when the angular link position and link velocity of the master and slave robot coincide for all $t \geq 0$, or asymptotically for $t \rightarrow \infty$. One can introduce synchronization indices as the link variables of interest

$$
J(q(t), \dot{q}(t))=\left[\begin{array}{ll}
q(t)^{T} & \dot{q}(t)^{T}
\end{array}\right]
$$

with the corresponding set of functionals

$$
f_{\text {sync }}\left(q_{m}, \dot{q}_{m}, q_{s}, \dot{q}_{s}\right)=\left\|J\left(q_{m}, \dot{q}_{m}\right)-J\left(q_{s}, \dot{q}_{s}\right)\right\|
$$

From Section 2.2.2 it follows that the reduced model for the flexible joint robot dynamics is given by (for $i=m, s$, the master and slave robot respectively)

$$
\begin{array}{ccc}
M_{i}\left(q_{i}\right) \ddot{q}_{i}+N_{i}\left(q_{i}, \dot{q}_{i}\right)+K_{i}\left(q_{i}-\theta_{i}\right)= & 0 \\
J_{i} \ddot{\theta}_{i}+K_{i}\left(\theta_{i}-q_{i}\right)+B_{v, i} \dot{\theta}_{i}= & \tau_{i} \\
N_{i}\left(q_{i}, \dot{q}_{i}\right)=C_{i}\left(q_{i}, \dot{q}_{i}\right) \dot{q}_{i}+g_{i}\left(q_{i}\right) &
\end{array}
$$

Notice that the dynamic model of the flexible joint robot $(4.1-4.3)$ accounts for viscous friction phenomena. In this case the viscous friction induces damping in the dynamic model, such that the stability properties of the proposed synchronization scheme can be proved. Other kinds of friction phenomena may be treated as in the case of rigid joint robots, i.e. these might be compensated separately. In order to include some other friction phenomena different than static viscous friction it is possible to consider the dynamic model given by (2.32), i.e.

$$
\begin{aligned}
& M_{i}\left(q_{i}\right) \ddot{q}_{i}+N_{i}\left(q_{i}, \dot{q}_{i}\right)+K_{i}\left(q_{i}-\theta_{i}\right)=0 \\
& J_{i} \ddot{\theta}+K_{i}\left(\theta_{i}-q_{i}\right)+B_{v, i} \dot{\theta}_{i}+f_{i}\left(\dot{\theta}_{i}\right)=\tau_{e}
\end{aligned}
$$

with the friction forces $f_{i}\left(\dot{\theta}_{i}\right)$ modelled similar to $(2.30)$, i.e.

$$
f_{i}\left(\dot{\theta}_{i}\right)=B_{f 1, i}\left(1-\frac{2}{1+e^{2 w_{1, i} \dot{\theta}_{i}}}\right)+B_{f 2, i}\left(1-\frac{2}{1+e^{2 w_{2, i} \dot{\theta}_{i}}}\right)
$$


Several authors have addressed the problem of tracking in rigid-link flexible-joint robots assuming the full state available, (De Luca 1998), (Lozano and Brogliato 1992), (Qu 1995). But there are few controllers that assume partial knowledge of the state, see (Lim et al. 1997), (Dixon et al. 2000). They proposed adaptive controllers based on backstepping and filters requiring only link and actuator position measurements. In our setup we assume only link position measurements, which makes our synchronization controller quite attractive since not velocity measuring equipment is required. The chapter is organized as follows. A nominal controller, assuming that all signals

$\theta_{s}, \dot{\theta}_{s}, q_{m}, \dot{q}_{m}, q_{s}, \dot{q}_{s}$ are available, is introduced in Section 4.2. Section 4.3 presents an implementation of the nominal controller based on estimated values for $\theta_{s}, \dot{\theta}_{s}, \dot{q}_{m}, \dot{q}_{s}$, which are obtained by two observers. The synchronization closed loop system formed by the slave robot and the modified nominal controller and observers is obtained in Section 4.3.3, and the stability analysis is presented in Section 4.3.4. Section 4.4 summarizes a gain tuning procedure for the parameters in the controller and observers. A simulation study in a one degree of freedom master and slave robot is presented in Section 4.5. The chapter closes with some concluding remarks and discussions about the proposed synchronization controller.

\subsection{Synchronization controller based on state feed- back}

Assume that (4.1), (4.2) define the dynamics of two robots which are interconnected in a synchronization master slave scheme where the subindices $m, s$ identify the master and slave robot respectively. Based on inverse dynamics computation and according to De Luca and Lucibello (1998), the slave robot with dynamic model (4.1), (4.2) can be fully linearized and decoupled via the static feedback control law

$$
\begin{gathered}
\tau_{s}=K_{s}\left(\theta_{s}-q_{s}\right)+B_{v, s} \dot{\theta}_{s}+J_{s} K_{s}^{-1}\left[M_{s}\left(q_{s}\right) v(t)+\alpha\left(q_{s}, \dot{q}_{s}, \ddot{q}_{s}, q_{s}^{(3)}\right)\right] \\
\alpha\left(q_{s}, \dot{q}_{s}, \ddot{q}_{s}, q_{s}^{(3)}\right)=2 \dot{M}_{s}\left(q_{s}, \dot{q}_{s}\right) q_{s}^{(3)}+\left(\ddot{M}_{s}\left(q_{s}, \dot{q}_{s}, \ddot{q}_{s}\right)+K_{s}\right) \ddot{q}_{s}+\ddot{N}\left(q_{s}, \dot{q}_{s}, \ddot{q}_{s}, q_{s}^{(3)}\right)
\end{gathered}
$$

On the other hand (4.1) establishes a relation between $\ddot{q}_{s}, q_{s}^{(3)}$, and lower order variables $q_{s}, \dot{q}_{s}, \dot{\theta}_{s}$. From (4.1) it follows that

$$
\begin{aligned}
\ddot{q}_{s} & =-M_{s}^{-1}\left(q_{s}\right)\left(N\left(q_{s}, \dot{q}_{s}\right)+K_{s}\left(q_{s}-\theta_{s}\right)\right) \\
q_{s}^{(3)} & =-M_{s}^{-1}\left(q_{s}\right)\left(\dot{M}_{s}\left(q_{s}, \dot{q}_{s}\right) \ddot{q}_{s}+\dot{N}\left(q_{s}, \dot{q}_{s}, \ddot{q}_{s}\right)+K_{s}\left(\dot{q}_{s}-\dot{\theta}_{s}\right)\right)
\end{aligned}
$$

Therefore (4.6) can be written as function of $q_{s}, \dot{q}_{s}, \theta_{s}, \dot{\theta}_{s}$, i.e.

$$
\begin{gathered}
\tau_{s}=K_{s}\left(\theta_{s}-q_{s}\right)+\beta\left(q_{s}, \dot{q}_{s}, \theta_{s}, \dot{\theta}_{s}\right)+\varphi\left(q_{s}\right) v(t) \\
\beta\left(q_{s}, \dot{q}_{s}, \theta_{s}, \dot{\theta}_{s}\right)=B_{v, s} \dot{\theta}_{s}+J_{s} K_{s}^{-1} \alpha\left(q_{s}, \dot{q}_{s}, \ddot{q}_{s}, q_{s}^{(3)}\right) \\
\varphi\left(q_{s}\right)=J_{s} K_{s}^{-1} M_{s}\left(q_{s}\right)
\end{gathered}
$$

Applying the control law (4.6) to the slave robot (4.1), (4.2) yields the linear decoupled closed loop system

$$
q_{s}^{(4)}=v(t)
$$


To ensure synchronization between the slave and the master robot an additional feedback for $v(t)$ is proposed as

$$
v(t)=q_{m}^{(4)}-K_{3} e^{(3)}-K_{2} \ddot{e}-K_{1} \dot{e}-K_{0} e
$$

with the synchronization errors defined by

$$
e=q_{s}-q_{m}, \quad \dot{e}=\dot{q}_{s}-\dot{q}_{m}
$$

where $K_{i} \in \mathbb{R}^{n}$ are gain matrices, and $q_{m}$ is the master link position trajectory, that has to be at least four times continuously differentiable, i.e. $q_{m} \in C^{4}$.

Clearly, there exist general choices for the gain matrices $K_{i}$ such that the closed loop systems is stable, but for simplicity we assume that $K_{i}=k_{i} I_{n}, i=0,1,2,3$, with $k_{i}$ positive scalars. Then, it is straightforward to conclude that the synchronization error $e$ will tend to zero exponentially, if the scalars $k_{i}, i=0,1,2,3$ are chosen such that the polynomial $s^{4}+k_{3} s^{3}+k_{2} s^{2}+k_{1} s+k_{0}$ is Hurwitz.

\subsection{Synchronization controller based on estimated variables}

As stated in the introduction of this chapter, it is assumed that only the link positions $q_{m}, q_{s}$ are measured, therefore (4.6) cannot be implemented. Moreover, also $v(t)$ in (4.12) cannot be implemented since it depends on higher order derivatives of $q_{s}, q_{m}$.

Let $\widehat{q}_{s}, \widehat{\dot{q}}_{s}, \widehat{\theta}_{s}$, and $\widehat{\dot{\theta}}_{s}$ denote estimated values for the link and rotor variables $q_{s}, \dot{q}_{s}, \theta_{s}, \dot{\theta}_{s}$, and let $\widehat{v}(t)$ denote the implementation of $v(t)$, given by (4.12), based on estimated synchronization errors. Assuming that $\widehat{q}_{s}, \widehat{\dot{q}}_{s}, \widehat{\theta}_{s}, \widehat{\dot{\theta}}_{s}$ and $\widehat{v}(t)$ are available the linearizing feedback control (4.10) can be modified as

$$
\tau_{s}=K_{s}\left(\widehat{\theta}_{s}-\widehat{q}_{s}\right)+\beta\left(q_{s}, \widehat{\dot{q}}_{s}, \widehat{\theta}_{s}, \widehat{\dot{\theta}}_{s}\right)+\varphi\left(q_{s}\right) \widehat{v}(t)
$$

with $\beta\left(q_{s}, \widehat{\dot{q}}_{s}, \widehat{\theta}_{s}, \widehat{\dot{\theta}}_{s}\right)$ given by

$$
\begin{gathered}
\beta\left(q_{s}, \widehat{\dot{q}}_{s}, \widehat{\theta}_{s}, \widehat{\dot{\theta}}_{s}\right)=B_{v, s} \widehat{\dot{\theta}}_{s}+J_{s} K_{s}^{-1} \alpha\left(q_{s}, \widehat{\dot{q}}_{s}, \widehat{\ddot{q}}_{s}, \widehat{q_{s}^{(3)}}\right) \\
\alpha\left(q_{s}, \widehat{\dot{q}}_{s}, \widehat{\ddot{q}}_{s}, \widehat{q_{s}^{(3)}}\right)=2 \dot{M}_{s}\left(q_{s}, \widehat{\dot{q}}_{s}\right) \widehat{q_{s}^{(3)}}+\left(\ddot{M}_{s}\left(q_{s}, \widehat{\dot{q}}_{s}, \widehat{\ddot{q}}_{s}\right)+K_{s}\right) \widehat{\ddot{q}}_{s}+\ddot{N}\left(q_{s}, \widehat{\dot{q}}_{s}, \widehat{\ddot{q}}_{s}, \widehat{q_{s}^{(3)}}\right)
\end{gathered}
$$

where according to $(4.8),(4.9)$, estimates for $\ddot{q}_{s}, q_{s}^{(3)}$ are given by

$$
\begin{aligned}
\widehat{\ddot{q}}_{s} & =-M_{s}^{-1}\left(q_{s}\right)\left(N\left(q_{s}, \widehat{\dot{q}}_{s}\right)+K_{s}\left(\widehat{q}_{s}-\widehat{\theta}_{s}\right)\right) \\
\widehat{q_{s}^{(3)}} & =-M_{s}^{-1}\left(q_{s}\right)\left(\dot{M}_{s}\left(q_{s}, \widehat{\dot{q}}_{s}\right) \widehat{\ddot{q}}_{s}+\dot{N}\left(q_{s}, \widehat{\dot{q}}_{s}, \widehat{\ddot{q}}_{s}\right)+K_{s}\left(\widehat{\dot{q}}_{s}-\widehat{\dot{\theta}}_{s}\right)\right)
\end{aligned}
$$

\subsubsection{An observer for the synchronization errors $e, \dot{e}$}

Based on the work of Berghuis and Nijmeijer (1994), we propose an implementation of the controller $v(t)$, given by (4.12), based on estimates, of the form

$$
\widehat{v}(t)=-K_{3} \dot{w}_{2}-K_{2} \dot{w}_{1}-K_{1} \dot{\hat{e}}-K_{0} \widehat{e}
$$


where $\widehat{e}, \dot{\widehat{e}}, \dot{w}_{1}, \dot{w}_{2}$ represent estimates for $e, \dot{e}, \ddot{e}, e^{(3)}$ respectively. They are obtained by the observer

$$
\begin{array}{lc}
\dot{\hat{e}}=w_{1}+\Gamma_{1} \widetilde{e}, \quad \dot{w}_{1}=w_{2}+\Gamma_{2} \widetilde{e} \\
\dot{w}_{2}=w_{3}+\Gamma_{3} \widetilde{e}, \quad \dot{w}_{3}=\Gamma_{4} \widetilde{e}
\end{array}
$$

with the estimation synchronization errors defined by

$$
\widetilde{e}=e-\widehat{e}, \quad \dot{\widetilde{e}}=\dot{e}-\dot{\widehat{e}}
$$

and $\Gamma_{i} \in \mathbb{R}^{n \times n}, i=1,2,3,4$ diagonal positive definite gain matrices.

\subsubsection{An observer for the slave variables $q_{s}, \dot{q}_{s}, \theta_{s}, \dot{\theta}_{s}$}

Based on the dynamic model (4.1), (4.2), we propose the full state nonlinear Luenberger observer

$$
\begin{aligned}
& \frac{d}{d t} \widehat{q}_{s}=\widehat{\dot{q}}_{s}+\mu_{1} \widetilde{q}_{s} \\
& \frac{d}{d t} \widehat{\dot{q}}_{s}=-M_{s}^{-1}\left(q_{s}\right)\left(N\left(q_{s}, \widehat{\dot{q}}_{s}\right)+K_{s}\left(\widehat{q}_{s}-\widehat{\theta}_{s}\right)\right)+\mu_{2} \widetilde{q}_{s} \\
& \frac{d}{d t} \widehat{\theta}_{s}=\widehat{\dot{\theta}}_{s}+\mu_{3} \widetilde{q}_{s} \\
& \frac{d}{d t} \widehat{\dot{\theta}}_{s}=J_{s}^{-1}\left(\tau_{s}\left(q_{s}, \widehat{q}_{s}, \widehat{\dot{q}}_{s}, \widehat{\theta}_{s}, \widehat{\dot{\theta}}_{s}\right)-K_{s}\left(\widehat{\theta}_{s}-\widehat{q}_{s}\right)-B_{v, s} \widehat{\dot{\theta}}_{s}\right)+\mu_{4} \widetilde{q}_{s}
\end{aligned}
$$

where $\widehat{q}_{s}, \widehat{\dot{q}}_{s}, \widehat{\theta}_{s}, \widehat{\dot{\theta}}_{s}$ correspond to the estimates of $q_{s}, \dot{q}_{s}, \theta_{s}, \dot{\theta}_{s}$, and $\mu_{1}, \mu_{2}, \mu_{3}, \mu_{4} \in$ $\mathbb{R}^{n \times n}$ are diagonal positive definite gain matrices. The joint estimation errors are defined by

$$
\begin{array}{ll}
\widetilde{q}_{s}=q_{s}-\widehat{q}_{s}, & \widetilde{\dot{q}}_{s}=\dot{q}_{s}-\widehat{\hat{q}}_{s} \\
\widetilde{\theta}_{s}=\theta_{s}-\widehat{\theta}_{s}, & \tilde{\dot{\theta}}_{s}=\dot{\theta}_{s}-\hat{\dot{\theta}}_{s}
\end{array}
$$

Figure 4.2 shows a diagram of the proposed synchronization controller.

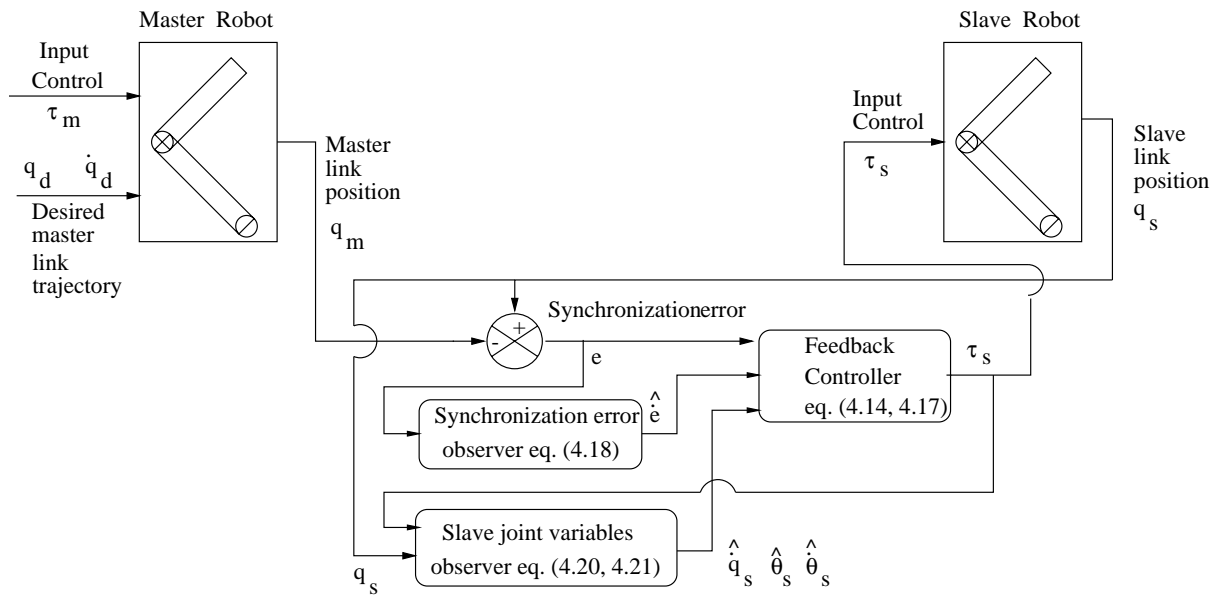

Figure 4.2: External synchronization of flexible joint robots. 


\subsubsection{Synchronization closed loop error dynamics}

In this section compact synchronization closed loop error dynamics, that are suitable for a straightforward Lyapunov stability analysis, are presented.

Lemma 4.1 Consider the master and slave flexible joint robots, which are described by (4.1 - 4.3), the slave robot in closed loop with the control law $\tau_{s}(4.14,4.17)$ and the observers (4.18), (4.20), and (4.21). In terms of $q_{s}, \dot{q}_{m}, \ddot{q}_{m}, q_{m}^{(3)}$, the joint estimation errors $\widetilde{q}_{s}, \dot{\tilde{q}}_{s}, \widetilde{\theta}_{s}, \dot{\tilde{\theta}}_{s}$, the synchronization errors e, $\dot{e}, \ddot{e}, e^{(3)}$ and the estimation synchronization errors $\widetilde{e}, \dot{\widetilde{e}}, \ddot{\widetilde{e}}, \widetilde{e}^{(3)}$, the synchronization closed loop error dynamics are given by

$$
\begin{aligned}
& e^{(4)}+K_{3} e^{(3)}+K_{2} \ddot{e}+K_{1} \dot{e}+K_{0} e=q_{m}^{(4)}+K_{3}\left(\widetilde{e}^{(3)}+\Gamma_{1} \ddot{\widetilde{e}}+\Gamma_{2} \dot{\widetilde{e}}\right) \\
& +K_{2}\left(\ddot{\widetilde{e}}+\Gamma_{1} \dot{\tilde{e}}\right)+K_{1} \dot{\widetilde{e}}+K_{0} \widetilde{e} \\
& -M_{s}^{-1}\left(q_{s}\right) \Phi\left(q_{s}, \dot{q}_{m}, \ddot{q}_{m}, q_{m}^{(3)}, \dot{e}, \ddot{e}, e^{(3)}, \widetilde{q}_{s}, \dot{\widetilde{q}}_{s}, \widetilde{\theta}_{s}, \dot{\widetilde{\theta}}_{s}\right) \\
& \widetilde{e}^{(4)}+\left(\Gamma_{1}-K_{3}\right) \widetilde{e}^{(3)}+\left(\Gamma_{2}-K_{3} \Gamma_{1}-K_{2}\right) \ddot{\widetilde{e}}+\left(\Gamma_{3}-K_{3} \Gamma_{2}-K_{2} \Gamma_{1}-K_{1}\right) \dot{\widetilde{e}} \\
& +\left(\Gamma_{4}-K_{0}\right) \widetilde{e}=q_{m}^{(4)}-K_{3} e^{(3)}-K_{2} \ddot{e}-K_{1} \dot{e}-K_{0} e \\
& -M_{s}^{-1}\left(q_{s}\right) \Phi\left(q_{s}, \dot{q}_{m}, \ddot{q}_{m}, q_{m}^{(3)}, \dot{e}, \ddot{e}, e^{(3)}, \widetilde{q}_{s}, \dot{\widetilde{q}}_{s}, \widetilde{\theta}_{s}, \dot{\tilde{\theta}}_{s}\right) \\
& \ddot{\widetilde{q}}_{s}=-M_{s}^{-1}\left(q_{s}\right)\left(\left(2 C_{s}\left(q_{s}, \dot{e}+\dot{q}_{m}\right)-C_{s}\left(q_{s}, \dot{\widetilde{q}}_{s}+\mu_{1} \widetilde{q}_{s}\right)\right)\left(\dot{\widetilde{q}}_{s}+\mu_{1} \widetilde{q}_{s}\right)\right. \\
& \left.+K_{s}\left(\widetilde{q}_{s}-\widetilde{\theta}_{s}\right)\right)-\mu_{1} \dot{\widetilde{q}}_{s}-\mu_{2} \widetilde{q}_{s} \\
& \ddot{\tilde{\theta}}_{s}=-J_{s}^{-1} K_{s}\left(\widetilde{\theta}_{s}-\widetilde{q}_{s}\right)-J_{s}^{-1} B_{v, s}\left(\dot{\tilde{\theta}}_{s}+\mu_{3} \widetilde{q}_{s}\right)-\mu_{3} \dot{\tilde{q}}_{s}-\mu_{4} \widetilde{q}_{s}
\end{aligned}
$$

Proof: See Appendix C.

Notice that the synchronization error dynamics (4.23) and (4.24) resemble a linear system which is perturbed by $\Phi$. Also note that the error dynamics (4.25) can be considered as a non-linear perturbed system.

Lemma 4.2 Define states $x_{1}, \ldots, x_{8} \in \mathbb{R}^{n}$ as $x_{1}=e, x_{2}=\dot{e}, x_{3}=\ddot{e}, x_{4}=e^{(3)}, x_{5}=$ $\widetilde{e}, x_{6}=\dot{\widetilde{e}}, x_{7}=\ddot{\widetilde{e}}, x_{8}=\widetilde{e}^{(3)}$, and $y \in \mathbb{R}^{4 n}$ as $y_{1}=\widetilde{q}_{s}, y_{2}=\dot{\widetilde{q}}_{s}, y_{3}=\widetilde{\theta}_{s}, y_{4}=\dot{\tilde{\theta}}_{s}$, and let the state vectors $x$ and $y$ be given by $x=\left[\begin{array}{llllllll}x_{1}^{T} & x_{2}^{T} & x_{3}^{T} & x_{4}^{T} & x_{5}^{T} & x_{6}^{T} & x_{7}^{T} & x_{8}^{T}\end{array}\right]^{T}$ and $y=\left[\begin{array}{llll}y_{1}^{T} & y_{2}^{T} & y_{3}^{T} & y_{4}^{T}\end{array}\right]^{T}$.

Then the synchronization closed loop error dynamics (4.23), (4.24), and (4.25) can be written as

$$
\begin{aligned}
& \dot{x}=A x-B M_{s}^{-1}\left(q_{s}\right) \Phi\left(q_{s}, \dot{q}_{m}, \ddot{q}_{m}, q_{m}^{(3)}, x_{2}, x_{3}, x_{4}, y_{1}, y_{2}, y_{3}, y_{4}\right)+B q_{m}^{(4)} \\
& \dot{y}=f\left(q_{s}, \dot{q}_{m}, x, y\right)
\end{aligned}
$$

where the matrices $A \in \mathbb{R}^{8 n \times 8 n}, B \in \mathbb{R}^{8 n \times n}$ are given by 


$$
A=\left[\begin{array}{cccccccc}
0 & I_{n} & 0 & 0 & 0 & 0 & 0 & 0 \\
0 & 0 & I_{n} & 0 & 0 & 0 & 0 & 0 \\
0 & 0 & 0 & I_{n} & 0 & 0 & 0 & 0 \\
-K_{0} & -K_{1} & -K_{2} & -K_{3} & K_{0} & \pi_{1} & \pi_{2} & K_{3} \\
0 & 0 & 0 & 0 & 0 & I_{n} & 0 & 0 \\
0 & 0 & 0 & 0 & 0 & 0 & I_{n} & 0 \\
0 & 0 & 0 & 0 & 0 & 0 & 0 & I_{n} \\
-K_{0} & -K_{1} & -K_{2} & -K_{3} & K_{0}-\Gamma_{4} & \pi_{1}-\Gamma_{3} & \pi_{2}-\Gamma_{2} & K_{3}-\Gamma_{1}
\end{array}\right]
$$

with $0, I_{n} \in \mathbb{R}^{n \times n}$ the zero and identity matrices.

The nonlinear vector function $f\left(q_{s}, \dot{q}_{m}, x, y\right)$ is given by

$$
f\left(q_{s}, \dot{q}_{m}, x, y\right)=\left[\begin{array}{l}
y_{2} \\
-M_{s}^{-1}\left(q_{s}\right)\left(2 C_{s}\left(q_{s}, x_{2}+\dot{q}_{m}\right)-C_{s}\left(q_{s}, y_{2}+\mu_{1} y_{1}\right)\right) \\
\times\left(y_{2}+\mu_{1} y_{1}\right)-M_{s}^{-1}\left(q_{s}\right) K_{s}\left(y_{1}-y_{3}\right)-\mu_{1} y_{2}-\mu_{2} y_{1} \\
y_{4} \\
-J_{s}^{-1} K_{s}\left(y_{3}-y_{1}\right)-J_{s}^{-1} B_{v, s}\left(y_{4}+\mu_{3} y_{1}\right)-\mu_{3} y_{2}-\mu_{4} y_{1}
\end{array}\right]
$$

Proof: This follows from simple substitution of the states $x$ and $y$.

\subsubsection{Stability analysis}

The stability analysis is based on a Lyapunov function, whose derivative can be bounded in terms of the closed loop errors. It is proven that the bound is negative in an annulus around the origin. In order to derive bounds on the derivative of the Lyapunov function the following assumption is required.

Assumption 4.3 The signals $\dot{q}_{m}, \ddot{q}_{m}, q_{m}^{(3)}, q_{m}^{(4)}$ are bounded for all $t \in\left[t_{0}, \infty\right)$, therefor there exist $V_{M}, A_{M}, D_{M}$ and $E_{M}$ such that

$$
\begin{aligned}
& \sup _{t}\left\|\dot{q}_{m}\right\|=V_{M}<\infty, \quad \sup _{t}\left\|\ddot{q}_{m}\right\|=A_{M}<\infty \\
& \sup _{t}\left\|q_{m}^{(3)}\right\|=D_{M}<\infty, \quad \sup _{t}\left\|q_{m}^{(4)}\right\|=E_{M}<\infty
\end{aligned}
$$

In practice, it is often not difficult to obtain the master trajectories bounds (4.30) on the basis of the desired trajectories $q_{d}(t)$ and its derivatives, although due to friction effects, tracking errors, etc., the actual motion of the master robot may differ from its desired motion. Also the bounds $V_{M}, A_{M}, D_{M}$, and $E_{M}$ can be obtained by considering the structural limitations of the robots, such as maximum velocities and accelerations of the motors.

On the other hand, for the sake of simplicity the following assumption is considered. 
Assumption 4.4 All gains in the control (4.14), (4.17), and the observer (4.18) are a positive multiple of the unit matrix, i.e. of the form $K=k I$, where $k$ is a positive scalar associated to the respective gain and $I$ denotes the identity matrix of appropriate dimensions.

Based on the above assumptions, the main result of this chapter is formulated as follows.

Theorem 4.5 Consider the master and slave flexible joint robots, which are described by $\left(4.1\right.$ - 4.3), the slave robot in closed loop with the control law $\tau_{s}(4.14,4.17)$ and the observers (4.18), (4.20) and (4.21).

If the gain matrices $K_{i}$ and $\Gamma_{j}, i=0,1,2,3, j=1,2,3,4$ are chosen such that $A$, given by (4.27), is Hurwitz, and additionally the minimum and maximum eigenvalues of the gains $\mu_{l}, l=1,2,3,4$, i.e. $\mu_{l m}$ and $\mu_{l M}$, satisfy

$$
\begin{array}{r}
\mu_{1 m}>\max \left\{0,2 M_{s m}^{-1}\left(\lambda_{0}-C_{M} V_{M}\right),\left(2 \lambda_{0} M_{s m}\right)^{-1}\right. \\
\left.\times\left(4 \lambda_{0}^{2}-M_{s m} K_{s m}-2 C_{M} V_{M}\left(2 \lambda_{0}+M_{s m} \mu_{1 M}\right)+M_{s m} M_{s M} \mu_{2 M}\right)\right\} \\
\mu_{2 m}>\max \left\{0,-a_{31}^{-1} a_{30},-a_{41}^{-1} a_{40}\right\} \\
\mu_{3 m}>\max \left\{0, \mu_{33_{-}}, \mu_{34_{-}}\right\}, \quad \mu_{3 M}<\min \left\{\mu_{33}^{-}, \mu_{34}^{-}\right\} \\
\mu_{4 m}>\max \left\{0, J_{s m}^{-1}\left(4 \eta_{0}^{2}-J_{s m} K_{s m}-2 \eta_{0} B_{v, s M}+J_{s m} \mu_{4 M}\right)\right\}
\end{array}
$$

where $M_{s m}$ and $J_{s m}$ are the minimum eigenvalue of the link and motor rotor inertia matrices, and the scalars $\lambda_{0}, \eta_{0}, \mu_{33_{-}}, \mu_{34_{-}}, \mu_{33}^{-}, \mu_{34}^{-}, a_{30}, a_{31}, a_{40}, a_{41}$ are defined in the gain tuning procedure in Section 4.4. Then the joint estimation errors $\widetilde{q}_{s}, \dot{\tilde{q}}_{s}, \widetilde{\theta}_{s}, \dot{\tilde{\theta}}_{s}$, the synchronization errors $e, \dot{e}, \ddot{e}, e^{(3)}$ and the estimation synchronization errors $\widetilde{e}$, $\dot{\widetilde{e}}, \ddot{\widetilde{e}}, \widetilde{e}^{(3)}$ are semi-globally uniformly ultimately bounded.

Proof: The proof is divided in three parts. First the candidate Lyapunov function and conditions for positive definitiveness are presented. In the second part it is shown that the derivative of the Lyapunov function along (4.26) is bounded, and finally in the third part sufficient conditions for negative definiteness are formulated.

Remark 4.6 Notice that (4.31) establishes a relation between the minimum and maximum eigenvalue of the gain $\mu_{1}$, and thus they cannot be assigned arbitrarily. The same holds for the gain $\mu_{4}$. Also notice that the maximum eigenvalue of $\mu_{3}$ is bounded by (4.33), so it cannot be arbitrarily large.

\section{Lyapunov function}

Consider the system defined by (4.26), and take as a candidate Lyapunov function $V(x, y)$, given by

$$
V(x, y)=x^{T} P_{x} x+\frac{1}{2} y^{T} P_{y}(y) y
$$

where the positive definite symmetric matrix $P_{x}$ is the solution of the Lyapunov equation $P_{x} A+A^{T} P_{x}=-Q_{x}$, for any given symmetric positive definite matrix $Q_{x}$, and $P_{y}(y)$ is given by 


$$
P_{y}(y)=\left[\begin{array}{cc}
{\left[K_{s}+2 \lambda_{0} \mu_{1}+\beta_{1} I_{n}\right.} & 2 \lambda_{0} I_{n} \\
2 \lambda_{0} I_{n} & M_{s}\left(q_{s}\right)
\end{array}\right] \quad\left[\begin{array}{cc}
0 \\
0
\end{array}\right.
$$

with $\beta_{1}, \beta_{2}$ scalars to be determined, and $\eta\left(y_{3}\right)$ defined by

$$
\eta\left(y_{3}\right):=\frac{\eta_{0}}{1+\left\|y_{3}\right\|}
$$

$\lambda_{0}, \eta_{0}>0$ are scalar constants. Then for all $y_{3}=\widetilde{\theta}_{s}, y_{4}=\dot{\tilde{\theta}}_{s}$, it holds that

$$
0<\left\|\eta\left(y_{3}\right)\right\|<\eta_{0}, \quad \dot{\eta}\left(y_{3}\right) y_{4}^{T} y_{3} \leq \eta_{0}\left\|y_{4}\right\|^{2}
$$

Existence of $P_{x}$ is guaranteed if $A$, which is given by (4.27), is Hurwitz, see (Khalil 1996). By Assumption 4.4, a sufficient condition for $A$ being Hurwitz is that the scalar polynomial $\lambda(s)$, given by

$$
\begin{aligned}
\lambda(s)= & s^{8}+\gamma_{1} s^{7}+\gamma_{2} s^{6}+\gamma_{3} s^{5}+\left(\gamma_{4}+k_{1} \gamma_{1}+k_{2} \gamma_{2}+k_{3} \gamma_{3}\right) s^{4} \\
& +\left(k_{0} \gamma_{1}+k_{1} \gamma_{2}+k_{2} \gamma_{3}+k_{3} \gamma_{4}\right) s^{3}+\left(k_{0} \gamma_{2}+k_{1} \gamma_{3}+k_{2} \gamma_{4}\right) s^{2} \\
& +\left(k_{0} \gamma_{3}+k_{1} \gamma_{4}\right) s+k_{0} \gamma_{4}
\end{aligned}
$$

is Hurwitz. $k_{i}, \gamma_{j}$ are the scalars associated to the gains $K_{i}$ and $\Gamma_{j}, i=0,1,2,3$, $j=1,2,3,4$.

Therefore, by choosing the gains $k_{i}$ and $\gamma_{j}, i=0,1,2,3, j=1,2,3,4$, it can be ensured that the matrix $A$ is Hurwitz, so there exists a unique positive symmetric matrix $P_{x}$, which satisfies $P_{x} A+A^{T} P_{x}=-Q_{x}$, for any given symmetric positive definite matrix $Q_{x}$.

On the other hand, sufficient conditions for positive definiteness of $P_{y}(y)$ are

$$
\begin{aligned}
\mu_{1 m} & >\frac{1}{2 \lambda_{0} M_{s m}}\left(4 \lambda_{0}^{2}-M_{s m} K_{s m}-M_{s m} \beta_{1}\right) \\
\mu_{4 m} & >\frac{1}{J_{s m}}\left(4 \eta_{0}^{2}-J_{s m} K_{s m}-J_{s m} \beta_{2}\right)
\end{aligned}
$$

Finally, positive definiteness of $P_{x}$ and $P_{y}(y)$ imply positive definiteness of $V(x, y)$ in (4.35). Moreover, there exist positive constants $P_{m}$ and $P_{M}$ such that

$$
\frac{1}{2} P_{m}\|\xi\|^{2} \leq V(x, y) \leq \frac{1}{2} P_{M}\|\xi\|^{2}
$$

where $\xi^{T}=\left[\begin{array}{ll}x^{T} & y^{T}\end{array}\right]$.

\section{Derivative of the Lyapunov function}

Along the error dynamics (4.26), the time derivative of $V$ in (4.35) is given by

$$
\begin{aligned}
\dot{V}= & -x^{T} Q_{x} x-x^{T} P_{x} B M_{s}^{-1} \Phi-\Phi^{T} M_{s}^{-1} B^{T} P_{x} x+y^{T} P_{y}(y) f(x, y)+ \\
& +\frac{1}{2} y^{T} \dot{P}_{y}\left(q_{s}, y\right) y+x^{T} P_{x} B q_{m}^{(4)}+q_{m}^{(4) T} B^{T} P_{x} x
\end{aligned}
$$

which can be written as

$$
\dot{V}=-\left[\begin{array}{ll}
x & y
\end{array}\right]^{T}\left[\begin{array}{ll}
Q_{x} & Q_{x y} \\
Q_{x y}^{T} & Q_{y}
\end{array}\right]\left[\begin{array}{l}
x \\
y
\end{array}\right]+\Omega\left(q_{s}, \dot{q}_{m}, \ddot{q}_{m}, q_{m}^{(3)}, q_{m}^{(4)}, x, y\right)
$$


with $Q_{x} \in \mathbb{R}^{8 n \times 8 n}$ the symmetric positive definite matrix $Q_{x}=-\left(P_{x} A+A^{T} P_{x}\right)$. $Q_{y} \in \mathbb{R}^{4 n \times 4 n}$ is given by

$$
\begin{aligned}
Q_{y}=\left[\begin{array}{cccc}
2 \lambda_{0}\left(M_{s}^{-1} K_{s}+\mu_{2}\right) & \alpha_{1} & \alpha_{2} & \alpha_{3} \\
\alpha_{1}^{T} & M_{s} \mu_{1}-2 \lambda_{0} & \alpha_{4} & \frac{1}{2} J_{s} \mu_{3} \\
\alpha_{2}^{T} & \alpha_{4}^{T} & 2 \eta\left(y_{3}\right) J_{s}^{-1} K_{s} & \alpha_{5} \\
\alpha_{3}^{T} & \frac{1}{2}\left(J_{s} \mu_{3}\right)^{T} & \alpha_{5}^{T} & B_{v, s}-2 \eta\left(y_{3}\right) I_{n}
\end{array}\right] \\
\alpha_{1}=-\frac{1}{2}\left(\beta_{1} I_{n}+M_{s} \mu_{2}\right) \\
\alpha_{2}=-\lambda_{0} M_{s}^{-1} K_{s}+\eta\left(y_{3}\right)\left(\mu_{4}+J_{s}^{-1} B_{v, s} \mu_{3}-J_{s}^{-1} K_{s}\right) \\
\alpha_{3}=\frac{1}{2}\left(B_{v, s} \mu_{3}+J_{s} \mu_{4}-K_{s}\right) \\
\alpha_{4}=\eta\left(y_{3}\right) \mu_{3}-\frac{1}{2} K_{s} \\
\alpha_{5}=\eta\left(y_{3}\right) J_{s}^{-1} B_{v, s}-\frac{1}{2}\left(\mu_{4}+\beta_{2} I_{n}\right)
\end{aligned}
$$

$Q_{x y} \in \mathbb{R}^{8 n \times 4 n}$ depends on the entries of $P_{x}$, and it is given by

$$
Q_{x y}=\left[\begin{array}{c}
P_{x 14}+P_{x 18} \\
P_{x 24}+P_{x 28} \\
P_{x 34}+P_{x 38} \\
P_{x 44}+P_{x 48} \\
P_{x 45}^{T}+P_{x 58} \\
P_{x 46}^{T}+P_{x 68} \\
P_{x 47}^{T}+P_{x 78} \\
P_{x 48}^{T}+P_{x 88}
\end{array}\right] M_{s}^{-1} K_{s} J_{s}^{-1}\left[\left(B_{v, s} \mu_{3}-K_{s}\right) \quad 0 \quad K_{s} \quad B_{v, s}\right]
$$

The scalar function $\Omega\left(q_{s}, \dot{q}_{m}, \ddot{q}_{m}, q_{m}^{(3)}, q_{m}^{(4)}, x, y\right)$ is locally Lipschitz in all its arguments and is given by

$$
\begin{aligned}
\Omega= & -x^{T} P_{x} B M_{s}^{-1}\left(\Phi+K_{s} J_{s}^{-1} K_{s}\left(y_{1}-y_{3}\right)-K_{s} J_{s}^{-1} B_{v, s}\left(y_{4}+\mu_{3} y_{1}\right)\right) \\
& +x^{T} P_{x} B q_{m}^{(4)}-\left(\Phi+K_{s} J_{s}^{-1} K_{s}\left(y_{1}-y_{3}\right)+q_{m}^{(4) T} B^{T} P_{x} x\right. \\
& \left.-K_{s} J_{s}^{-1} B_{v, s}\left(y_{4}+\mu_{3} y_{1}\right)\right)^{T} M_{s}^{-1} B^{T} P_{x} x \\
& -\left(2 \lambda_{0} y_{1}^{T} M_{s}^{-1}+y_{2}^{T}\right)\left(2 C_{s}\left(q_{s}, x_{2}+\dot{q}_{m}\right)-C_{s}\left(q_{s}, \mu_{1} y_{1}\right)\right)\left(y_{2}+\mu_{1} y_{1}\right) \\
& +2 \lambda_{0} y_{1}^{T} M_{s}^{-1} C_{s}\left(q_{s}, y_{2}\right)\left(y_{2}+\mu_{1} y_{1}\right)+y_{2}^{T} C_{s}\left(q_{s}, y_{2}\right) \mu_{1} y_{1}+2 \dot{\eta}\left(y_{3}\right) y_{4}^{T} y_{3}
\end{aligned}
$$

\section{Boundedness of $\dot{V}$}

The next lemma synthesizes a general bound for $\dot{V}$ given by (4.40).

Lemma 4.7 Consider $\dot{V}$ given by (4.40) and introduce the vectors $x_{N}, y_{N}$, and $\xi_{N}$

$$
\begin{aligned}
& x_{N}=\left[\begin{array}{lllllllll}
\left\|x_{1}\right\| & \left\|x_{2}\right\| & \left\|x_{3}\right\| & \left\|x_{4}\right\| & \left\|x_{5}\right\| & \left\|x_{6}\right\| & \left\|x_{7}\right\| & \left\|x_{8}\right\|
\end{array}\right]^{T} \\
& y_{N}=\left[\begin{array}{lllll}
\left\|y_{1}\right\| & \left\|y_{2}\right\| & \left\|y_{3}\right\| & \left\|y_{4}\right\|
\end{array}\right]^{T} \\
& \xi_{N}=\left[\begin{array}{ll}
x_{N}^{T} & y_{N}^{T}
\end{array}\right]^{T}
\end{aligned}
$$

Then, an upperbound for the time derivative of $V$ is given by 


$$
\dot{V} \leq-\xi_{N}^{T} R_{V} \xi_{N}+\Theta\left(V_{M}, A_{M}, D_{M}, E_{M}, \xi_{N}\right)
$$

with $V_{m}, A_{m}, D_{m}, E_{m}$ given by (4.30), $\Theta\left(V_{m}, A_{m}, D_{m}, E_{m}, \xi_{N}\right)$ a scalar function that contains products of at most 5th order in terms of the entries of $\xi_{N}$, and the matrix $R_{V}$ is given by

$$
R_{V}=\left[\begin{array}{cc}
R_{x} & R_{x y}\left(V_{M}, A_{M}, D_{M}\right) \\
R_{x y}^{T}\left(V_{M}, A_{M}, D_{M}\right) & R_{y}\left(V_{m}\right)
\end{array}\right]
$$

where $R_{x} \in \mathbb{R}^{8 \times 8}$ is a positive definite matrix. $R_{x}, R_{x y} \in \mathbb{R}^{8 \times 4}, R_{y} \in \mathbb{R}^{4 \times 4}$, and $\Theta$ are given in Appendix D.

Proof: See Appendix D.

The upperbound of $\dot{V}$ given by (4.45) is in terms of the entries of the vector $\xi_{N}$, so that it can be reduced to a function of the norm of $\xi_{N}$.

Lemma 4.8 In terms of the vector $\xi_{N}$ defined by (4.44), the upperbound of $\dot{V}$ given by (4.45) can be reduced to

$$
\dot{V} \leq\left\|\xi_{N}\right\|\left(r_{0}-r_{1}\left\|\xi_{N}\right\|+r_{2}\left\|\xi_{N}\right\|^{2}+r_{3}\left\|\xi_{N}\right\|^{3}+r_{4}\left\|\xi_{N}\right\|^{4}\right)
$$

where $r_{1}$ is the minimum eigenvalue of the matrix $R_{V}$ in (4.46), and the positive scalars $r_{0}\left(E_{M}\right), r_{2}\left(\mu_{1 M}, \mu_{3 M}\right), r_{3}\left(\mu_{1 M}\right), r_{4}\left(\mu_{1 M}\right)$ are obtained from the term

$\Theta\left(V_{M}, A_{M}, D_{M}, E_{M}, \xi_{N}\right)$, with $V_{M}, A_{M}, D_{M}, E_{M}$ the upperbounds for $\dot{q}_{m}, \ddot{q}_{m}, q_{m}^{(3)}$, and $q_{m}^{(4)}$, which are defined by (4.30).

Proof: This follows directly from (4.45) and the definition of $\xi_{N}$.

\section{Negative definiteness of $\dot{V}$}

Equation (4.47) establishes an upperbound for $\dot{V}$, and this upperbound is negative if and only if $r_{1}$ is positive and large enough to dominate the other terms.

The scalar $r_{1}$ is the minimum eigenvalue of the matrix $R_{V}(4.46)$, so, $r_{1}$ is positive if and only if $R_{V}$ is positive definite. Lemma 2.1 is useful to prove positive definiteness of $R_{V}$. Before applying Lemma 2.1, it is required to prove that the matrix $R_{y}$ is positive definite. The conditions to fulfill this requirement are summarized in the following proposition.

Proposition 4.9 Consider $R_{y} \in \mathbb{R}^{4 \times 4}$ defined in (4.46), and define scalars $\beta_{1}, \beta_{2}$ as

$$
\begin{aligned}
& \beta_{1}=2 C_{M} V_{M}\left(2 M_{s m}^{-1} \lambda_{0}+\mu_{1 M}\right)-M_{s M} \mu_{2 M} \\
& \beta_{2}=2 \eta_{0} J_{s m}^{-1} B_{v, s M}-\mu_{4 M}
\end{aligned}
$$

where $\star_{m}$ and $\star_{M}$ denote the minimum and maximum eigenvalue of the matrix $\star$.

Then sufficient conditions for positive definiteness of $R_{y}$ are

1. $\lambda_{0}>0$

2. $\mu_{1 m}>\max \left\{0,2 M_{s m}^{-1}\left(\lambda_{0}-C_{M} V_{M}\right)\right\}$

3. $0<\eta_{0}<\min \left\{\frac{B_{v, s m}}{4}, \Upsilon\right\}$, 


$$
\begin{aligned}
& \text { with } \Upsilon=\frac{1}{8 J_{s M}}\left(J_{s M} B_{v, s m}+\left(J_{s M}^{2} B_{v, s m}^{2}+8 J_{s M} J_{s m}^{2} K_{s m}\right)^{1 / 2}\right) \\
& \text { 4. } \mu_{3 m}>\max \left\{0, \mu_{33_{-}}, \mu_{34_{-}}\right\}, \mu_{3 M}<\min \left\{\mu_{33}^{-}, \mu_{34}^{-}\right\} \\
& \text {5. } \mu_{2 m}>\max \left\{0,-a_{31}^{-1} a_{30},-a_{41}^{-1} a_{40}\right\} \\
& \text { with } \mu_{33_{-}}, \mu_{34_{-}}, \mu_{33}^{-}, \mu_{34}^{-}, a_{30}, a_{31}, a_{40}, a_{41} \text { given in Appendix } E \text {. }
\end{aligned}
$$

Proof: See Appendix E.

The conditions listed in Theorem 4.5 clearly imply the conditions in the above proposition, therefore it can be ensured that $R_{y}$ is positive definite and then Lemma 2.1 can be used.

For $R_{V}$, given by (4.46), the condition (2.2) can be written as

$$
\left\|R_{x}^{-1 / 2} R_{x y} R_{y}^{-1 / 2}\right\|_{2}<1
$$

On the other hand, notice that $R_{x y}$ depends on the gains $\mu_{1}$ and $\mu_{3}$, but does not depend on $\mu_{2}, \eta_{0}, \lambda_{0}$. Then, if the gains $\mu_{1}, \mu_{3}$ have been chosen according to Proposition 4.9, it follows that $R_{x y}$ is only determined by $P_{x}$ (4.35), which on its turn is determined by $Q_{x}$ (4.40). By choosing $Q_{x}(4.40)$ and the minimum eigenvalue of the gain $\mu_{2}$ such that $\operatorname{det}\left(Q_{x}\right) \gg 1$ and $\operatorname{det}\left(R_{y}\right) \gg 1$, it then follows that the entries of $R_{x}^{-1 / 2} R_{x y} R_{y}^{-1 / 2}$ are small. As a result $\left\|R_{x}^{-1 / 2} R_{x y} R_{y}^{-1 / 2}\right\|_{2}<1$ can be ensured, and thus $R_{V}$, given by (4.46), is positive definite. Moreover the minimum eigenvalue of $R_{V}$, i.e. $r_{1}$, is proportional to the minimum eigenvalue of $\mu_{2}$, which implies that $r_{1}$ can be chosen such that it dominates the other terms in (4.47). To emphasize the last conclusion, notice that $r_{0}, r_{2}, r_{3}, r_{4}$ do not depend on the gain $\mu_{2}$.

Finally, notice that according to Section 4.3.4, Proposition 4.9, and the above paragraph, it follows that if conditions in the Theorem 4.5 are fulfilled, then the function $V$, given by (4.35), is a Lyapunov function with $\dot{V}<0$ in an annulus around the origin, denoted by $\beta_{c}$. Therefore the closed loop errors are uniformly ultimately bounded in this annulus $\beta_{c}$. The annulus $\beta_{c}$ in which $\dot{V}<0$ defines the convergence region, such that in this region the terms $r_{2}\left\|\xi_{N}\right\|^{2}, r_{3}\left\|\xi_{N}\right\|^{3}$, and $r_{4}\left\|\xi_{N}\right\|^{4}$ are small and thus dominated by $r_{1}\left\|\xi_{N}\right\|$.

Note that the uniform ultimate boundedness of the synchronization errors is only valid in the convergence region $\beta_{c}$, and thus the result is of local nature. Nevertheless $r_{1}$ is proportional to the gain $\mu_{2}$, and none of the coefficients $r_{0}, r_{2}, r_{3}, r_{4}$ depend on $\mu_{2}$. Therefore, by selection of $\mu_{2}$, the coefficient $r_{1}$ can be increased, which results in enlargement of the convergence region $\beta_{c}$. Thus, the uniform ultimate boundedness of the synchronization errors is semi-global.

The ultimate boundedness result is due to the absence of measurements of derivatives of the master trajectory $q_{m}$, specifically $q_{m}^{(4)}$ through the coefficient $r_{0}\left(E_{M}\right)$ in (4.47), therefore we have the following corollary.

Corollary 4.10 If set point regulation of the master robot is considered and the master robot controller is able to achieved steady state in finite time, then $\dot{q}_{m}(t)=0$, $\ddot{q}_{m}(t)=0, q_{m}^{(3)}(t)=0$, and $q_{m}^{(4)}(t)=0$ for $t \in\left(t_{2}, \infty\right)$, with $t_{2} \geq t_{0}$ the convergence 
time of the master robot trajectories. If additionally the conditions in Theorem 4.5 are satisfied, then the control law (4.14), and the observers (4.18), (4.20), and (4.21) yield local exponential convergence of the synchronization closed loop errors.

Proof: If $q_{m}^{(4)}(t)=0$ for $t \in\left(t_{2}, \infty\right), t_{2} \geq t_{0}$, then the upperbound for $q_{m}^{(4)}$ is zero for $t \in\left(t_{2}, \infty\right), t_{2} \geq t_{0}$, and thus from Assumption 4.3 it follows that

$$
E_{M}=\sup _{t \geq t_{2}}\left\|q_{m}^{(4)}\right\|=0
$$

which implies that $r_{0}\left(E_{M}\right)=0$ in (4.47). Therefore, if conditions in Theorem 4.5 are satisfied, then for $t \geq t_{2}$ (4.47) reduces to

$$
\dot{V} \leq\left\|\xi_{N}\right\|^{2}\left(-r_{1}+r_{2}\left\|\xi_{N}\right\|+r_{3}\left\|\xi_{N}\right\|^{2}+r_{4}\left\|\xi_{N}\right\|^{3}\right)
$$

with $r_{1}, r_{2}, r_{3}, r_{4}>0$, and $\dot{V} \leq 0$ in the convergence region $\beta_{c}$. As a consequence, there exist a positive scalar $\kappa$, such that $\dot{V}$ has an upperbound given by

$$
\dot{V} \leq-\kappa\left\|\xi_{N}\right\|^{2} \quad \text { for all } t \geq t_{2}
$$

From the last equation and (4.38), we conclude that there exist some constants $m^{*}, \rho>$ 0 , such that

$$
\left\|\xi_{N}(t)\right\|^{2} \leq m^{*} e^{-\rho t}\left\|\xi_{N}\left(t_{2}\right)\right\|^{2} \quad \text { for all } t \geq t_{2}
$$

and thus by the definition of $\xi_{N}(t)$ given by (4.44), it follows that the close loop errors are semi-globally exponentially stable with convergence region $\beta_{c}$.

Remark 4.11 The proposed synchronization controller (4.14, 4.17) is designed to guarantee synchronization between two robots. Nevertheless it can be used as a tracking controller by taking the desired trajectory $q_{d}(t)$ as the master robot trajectory $q_{m}(t)$. In case of tracking the desired trajectory $q_{d}(t)$ and its derivatives are known, such that $q_{m}^{(4)}=q_{d}^{(4)}$ can be included through the control (4.17). In such case the closed loop error (4.26) does not depend on $q_{m}^{(4)}=q_{d}^{(4)}$, and the stability analysis would result in $\dot{V}$ given by (4.49). Therefore, for tracking of a known desired reference $q_{d}(t)$ the proposed synchronization controller $(4.14,4.17)$ with $\widehat{v}(t)(4.17)$ modified as

$$
\widehat{v}(t)=q_{d}^{(4)}(t)-K_{3} \dot{w}_{2}-K_{2} \dot{w}_{1}-K_{1} \dot{\widehat{e}}-K_{0} \widehat{e}
$$

yields semi-global exponential convergence of the closed loop errors.

\subsection{Gain tuning procedure}

The gain tuning procedure can be summarized as follows

1. Choose the gains $k_{i}$ and $\gamma_{j}, i=0,1,2,3, j=1,2,3,4$ such that $\lambda(s)$ in (4.36) is Hurwitz.

2. Determine the bounds of the physical parameters $M_{s}\left(q_{s}\right), C_{s}\left(q_{s}, \dot{q}_{s}\right), g_{s}\left(q_{s}\right), B_{v, s}$ and their partial derivatives with respect to $q_{s}$.

3. Determine the bounds on the master trajectories $\dot{q}_{m}, \ddot{q}_{m}, q_{m}^{(3)}, q_{m}^{(4)}$. 
4. Choose $\lambda_{0}>0, \mu_{1 M}>0, \mu_{4 M}>0$ and a maximum bound for the maximum eigenvalue of $\mu_{2}$, i.e. $\mu_{2 M}$.

5. Choose $\mu_{1}$, such that $\mu_{1 m}>\max \left\{0,2 M_{s m}^{-1}\left(\lambda_{0}-C_{M} V_{M}\right),\left(2 \lambda_{0} M_{s m}\right)^{-1}\right.$ $\left.\times\left(4 \lambda_{0}^{2}-M_{s m} K_{s m}-4 C_{M} V_{M} \lambda_{0}-2 C_{M} V_{M} M_{s m} \mu_{1 M}+M_{s m} M_{s M} \mu_{2 M}\right)\right\}$

6. Choose $\eta_{0}$, such that $0<\eta_{0}<\min \left\{\frac{B_{v, s m}}{4}, \frac{1}{8 J_{s M}}\left(J_{s M} B_{v, s m}+\left(J_{s M}^{2} B_{v, s m}^{2}+8 J_{s M} J_{s m}^{2} K_{s m}\right)^{1 / 2}\right)\right\}$

7. Select $\mu_{3}$, such that $\mu_{3 m}>\max \left\{0, \mu_{33_{-}}, \mu_{34_{-}}\right\}, \mu_{3 M}<\min \left\{\mu_{33}^{-}, \mu_{34}^{-}\right\}$, with $\mu_{33_{-}}, \mu_{34_{-}}, \mu_{33}^{-}, \mu_{34}^{-}$as in Appendix E.

8. $\mu_{4 m}>\max \left\{0, J_{s m}^{-1}\left(4 \eta_{0}^{2}-J_{s m} K_{s m}-2 \eta_{0} B_{v, s M}+J_{s m} \mu_{4 M}\right)\right\}$

9. Choose $Q_{x}$ a symmetric positive definite block diagonal matrix, with $n \times n$ block entries, such that $\operatorname{det}\left(Q_{x}\right) \gg 1$.

10. Determine $P_{x}$ such that $P_{x} A+A^{T} P_{x}=-Q_{x}$

11. Choose $\mu_{2}$, such that $\mu_{2 m}>\max \left\{0,-a_{31}^{-1} a_{30},-a_{41}^{-1} a_{40}\right\}$, with $a_{30}, a_{31}, a_{40}$, and $a_{41}$ as in Appendix E, and $\mu_{2 m}$ large enough to ensure that $\left\|R_{x}^{-1 / 2} R_{x y} R_{y}^{-1 / 2}\right\|_{2}<1$.

\subsection{Simulation study}

The slave and master robot considered in the simulation consist of one rigid link with a flexible joint, rotating in a vertical plane. The dynamic model is given by

$$
\begin{aligned}
M_{i} \ddot{q}_{i}+K_{i}\left(q_{i}-\theta_{i}\right)+\frac{1}{2} m_{i} g l_{i} \sin \left(q_{i}\right) & =0 \\
J_{i} \ddot{\theta}_{i}+K_{i}\left(\theta_{i}-q_{i}\right)+B_{v, i} \dot{\theta}_{i} & =\tau_{i}
\end{aligned}
$$

the subindex $i=m, s$ denote the master and slave robot respectively. The parameters of the master and slave robot are listed in Table 4.1.

\begin{tabular}{|c|c|c|c|c|c|}
\hline & $M_{i}\left[\mathrm{Kg} \cdot \mathrm{m}^{2}\right.$ & $m_{i}[\mathrm{Kg} \cdot \mathrm{m}]$ & $K_{i}$ & $\left.\mathrm{Kg} \cdot \mathrm{m}^{2} / \mathrm{s}^{2}\right]$ \\
\hline master robot & \multicolumn{2}{|c|}{0.5} & \multicolumn{2}{|c|}{1.4} & 75 \\
\hline slave robot & \multicolumn{2}{|c|}{0.4} & \multicolumn{2}{|c|}{1.0} & 100 \\
\hline & $B_{v, i}$ & $\mathrm{Kg} \cdot \mathrm{m}^{2} / \mathrm{s}$ & $J_{i}$ & $\mathrm{Kg} \cdot \mathrm{m}^{2}$ & $l_{i}[\mathrm{~m}]$ \\
\hline master robot & 2 & 0.04 & 1.0 \\
\hline slave robot & 5 & 0.02 & 1.0 \\
\hline
\end{tabular}

Table 4.1: Master and slave robot parameters

The master robot is driven by the nominal control law given by (4.6) and (4.12), and the gains on (4.12) are chosen as $k_{0 m}=1, k_{1 m}=3, k_{2 m}=6, k_{3 m}=3$. The desired link master trajectory is

$$
q_{m d}(t)=1+0.5 \sin (t) \quad[\mathrm{rad}] .
$$

The scalar gains, involved in the slave synchronization controller (4.17), and the observers (4.18), (4.20), and (4.21) are chosen to be $k_{0}=65, k_{1}=40, k_{2}=10$, $k_{3}=4, \gamma_{1}=40, \gamma_{2}=700, \gamma_{3}=4000, \gamma_{4}=1000, \mu_{1}=1, \mu_{2}=5, \mu_{3}=1, \mu_{4}=5$. 
The initial conditions on the master robot, slave robot, and the observers given by (4.18), (4.20), and (4.21) are chosen as in Table 4.2.

\begin{tabular}{|c|c|c|c|c|}
\hline & $q_{i}[\mathrm{rad}]$ & $\dot{q}_{i}[\mathrm{rad} / \mathrm{s}]$ & $\theta_{i}[\mathrm{rad}]$ & $\dot{\theta}_{i}[\mathrm{rad} / \mathrm{s}]$ \\
\hline master robot & 1.0 & 0 & 1.1 & 0 \\
\hline \multirow[t]{2}{*}{ slave robot } & 0.5 & 0 & 0.51 & 0 \\
\hline & $\widehat{e} \quad[\mathrm{rad}]$ & $w_{1}[\mathrm{rad} / \mathrm{s}]$ & \begin{tabular}{l|l}
$w_{2}$ & $\mathrm{rad} / \mathrm{s}^{2}$
\end{tabular} & \begin{tabular}{l|l}
$w_{3}$ & $\mathrm{rad} / \mathrm{s}^{3}$
\end{tabular} \\
\hline \multirow[t]{2}{*}{ slave robot } & -0.1 & 0 & 0 & 0 \\
\hline & $\widehat{q}_{s}[\mathrm{rad}]$ & $\widehat{\dot{q}}_{s}[\mathrm{rad} / \mathrm{s}]$ & $\widehat{\theta}_{s}[\mathrm{rad}]$ & $\widehat{\dot{\theta}}_{s}[\mathrm{rad} / \mathrm{s}]$ \\
\hline slave robot & 0.4 & 0 & 0 & 0 \\
\hline
\end{tabular}

Table 4.2: Initial conditions in slave and master robot.

Figure 4.3(left) shows the master and slave robot position $q_{m}$ and $q_{s}$. The position synchronization error $e$ is shown in Figure 4.3(right). The estimation errors of the slave link and rotor position (observer 4.20 and 4.21) are presented in Figures 4.4.
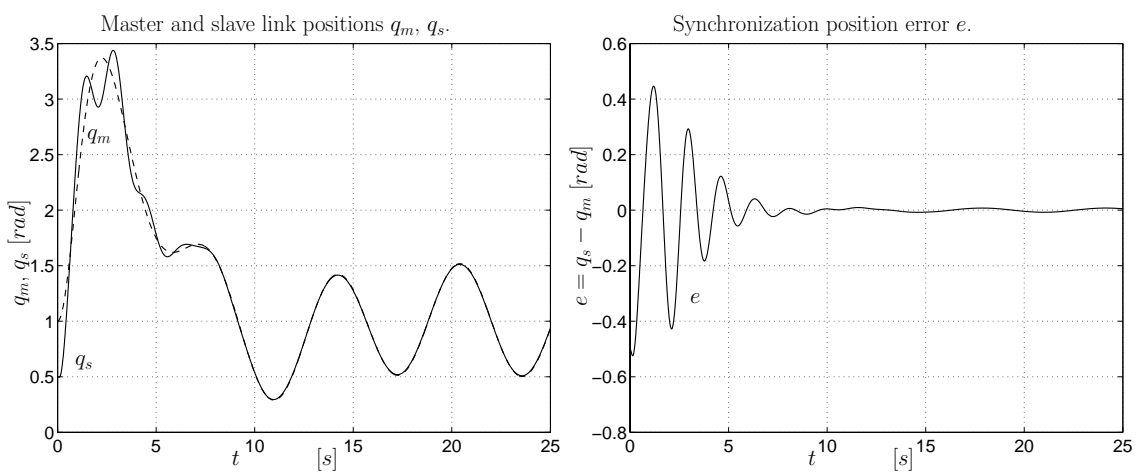

Figure 4.3: Master and slave link positions $q_{m}, q_{s}$, and synchronization error $e$.
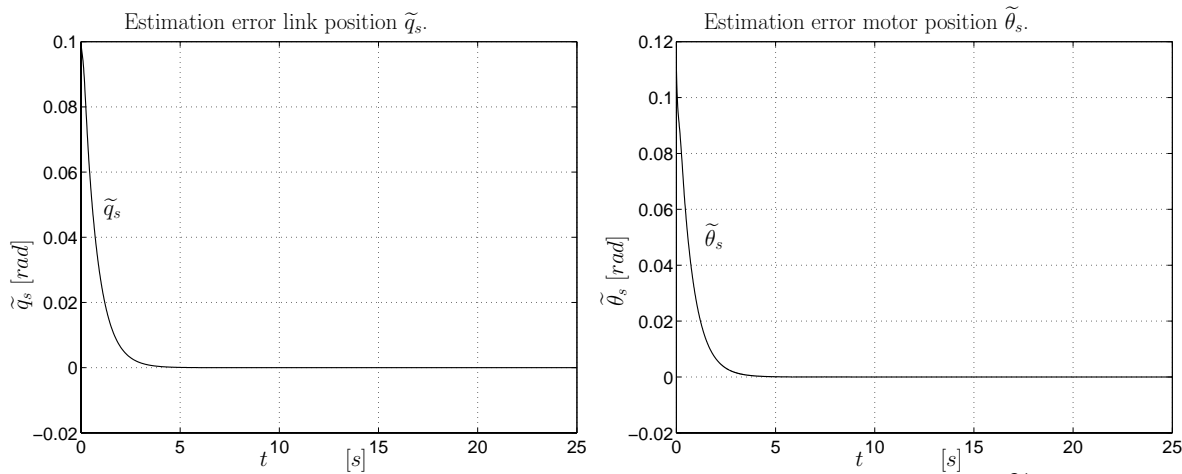

Figure 4.4: Estimation link and motor position error $\widetilde{q}_{s}, \widetilde{\theta}_{s}$. 
As it is shown in Figure 4.3, the synchronization error between the master and slave link position is stable and bounded after the transient period has finished. The same is concluded for the estimation errors in Figures 4.4.

In agreement with the stability analysis, the simulations have shown that the final bound of the errors depends on the gains $\mu_{2}$ and $K_{0}$. Meanwhile the transient behavior is mainly determined by $\mu_{1}, \mu_{3}$ and the gain $K_{1}$. This is due to the fact the $K_{1}$ defines the poles of the linear part $A x$ in (4.26), and $\mu_{1}, \mu_{3}$ weigh the effect of the estimation errors through the term $\Phi$ in (4.26). Therefore if $\mu_{1}, \mu_{3}$ are large, as well as the estimation errors, then the term $\Phi$ has a large influence in the synchronization error dynamics, and this large influence lasts until the estimation errors reach the vicinity of zero.

The simulation study shows that in order to minimize the peaks during the transient period, it is important to tune the gains in $\widehat{v}(t)$, given by (4.17), such that the polynomial (4.36) corresponds to an overdamped system. At the same time the gains $\mu_{1}, \mu_{3}$ should be set small to minimize the influence (through the term $\Phi$ ) of the estimation errors.

\subsection{Concluding remarks and discussion}

- The proposed synchronizing controller yields practical link position and velocity synchronization in the joint space. Position and velocity synchronization in the Cartesian space is obtained only if the length of the links of the slave robot are equal to the corresponding links in the master robot.

- Notice that the synchronization controller for flexible joint robots, given by (4.14), (4.17), and the observers (4.20) and (4.21), only requires measurements of the master robot link position $q_{m}$, not of the rotor position $\theta_{m}$. Therefore the master robot may or may not have flexible joints.

- Even without knowledge of the bounds considered in (4.31 - 4.34), the synchronization closed loop system can be made uniformly ultimately bounded, by selecting the control gains large enough. However, the value of the gains must not be so large such that the condition (4.33) is violated.

- The controller and observers (4.14), (4.20) and (4.21) are model based, nevertheless the stability analysis allows a straightforward robustness analysis for parametric uncertainties. At the same time adaptive versions of the proposed synchronization controller can be obtained by considering linear parametric representation of the dynamic model $(4.1,4.2)$.

- The proposed synchronization controller, linearization feedback (4.14) plus the observers (4.20) and (4.21), provides a systematic way of proving stability and boundedness of the synchronization closed loop system. This is a drawback of some other schemes for estimating velocities, such as numerical differentiation or low pass filters. For those techniques, in general, there do not exist formal stability proofs or a methodology to guarantee stability of the closed loop system.

- Static friction phenomena can be treated in a similar way as for synchronization of rigid joint robots, see Section 3.5. 


\section{Chapter 5}

\section{Mutual synchronization of rigid joint robots}

\subsection{Introduction}

This chapter addresses the problem of mutual synchronization of rigid joint robots, assuming only angular position measurements of the robots. Results of this chapter are reported in the article (Rodriguez-Angeles and Nijmeijer 2002a).

The general setup is as follows. Consider a multi-robot system formed by $p(p \geq 2)$ rigid joint robots together with a common desired trajectory for all of them, denoted by $q_{d}, \dot{q}_{d}$. Then, the mutual synchronization control problem can be formulated to design interconnections and controllers $\tau_{i}(\cdot)$ for all the robots in the system, such that the angular positions and velocities $q_{i}, \dot{q}_{i} \in \mathbb{R}^{n}$ of the $i$-th robot in the system are synchronized with respect to the common desired trajectory $q_{d}, \dot{q}_{d}$ and to the angular positions of the other robots $q_{j}, \dot{q}_{j} \in \mathbb{R}^{n},(j=1, \ldots, p, j \neq i)$.

It is assumed that the dynamic model of each robot is known and free of uncertainties and modelling errors. The major constraint to design the synchronization controller is that only the angular positions of all the robots are measured. Partial knowledge of the reference signals and the working (feedback) signals - only positions measurements of all the robots are assumed - demands the reconstruction of the missing required signals. This problem is solved by using nonlinear model-based observers. The estimated variables (velocities and accelerations) are used in a feedback loop, such that the overall mutual synchronization controller, i.e. feedback controller plus the observers, guarantees mutual synchronization of the multi-robot system.

Figure 5.1 shows a schematic representation of the mutual synchronization problem for the rigid robot case. Note that in this problem the angular positions are transmitted from and to all the robots in the system.

The cases of rigid joint robots with and without friction effects are addressed in this chapter. If friction effects are ideally compensated, then the robots can be considered as frictionless and can be modelled as (see Section 2.2.1)

$$
M_{i}\left(q_{i}\right) \ddot{q}_{i}+C_{i}\left(q_{i}, \dot{q}_{i}\right) \dot{q}_{i}+g_{i}\left(q_{i}\right)=\tau_{i}, \quad i=1, \ldots, p
$$




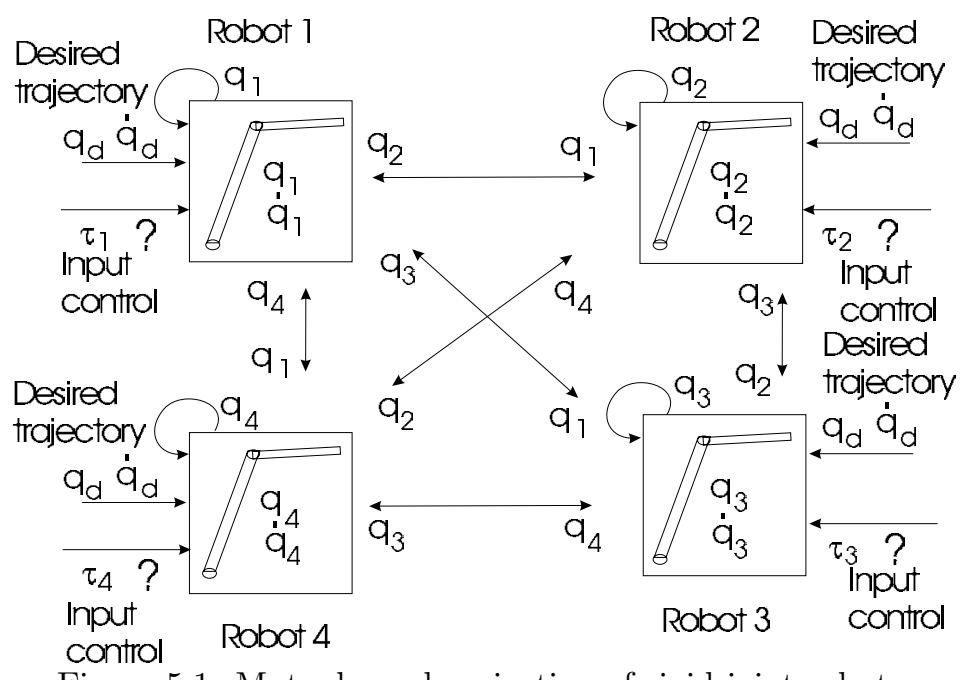

Figure 5.1: Mutual synchronization of rigid joint robots.

while, when friction forces $f_{i}\left(\dot{q}_{i}\right)$ are considered the model changes to

$$
M_{i}\left(q_{i}\right) \ddot{q}_{i}+C_{i}\left(q_{i}, \dot{q}_{i}\right) \dot{q}_{i}+g_{i}\left(q_{i}\right)+f_{i}\left(\dot{q}_{i}\right)=\tau_{i}
$$

in this chapter the particular friction model $f_{i}\left(\dot{q}_{i}\right)$ introduced in Section 2.2 .4 is considered

$$
f_{i}\left(\dot{q}_{i}\right)=B_{v, i} \dot{q}_{i}+B_{f 1, i}\left(1-\frac{2}{1+e^{2 w_{1, i} \dot{q}_{i}}}\right)+B_{f 2, i}\left(1-\frac{2}{1+e^{2 w_{2, i} \dot{q}_{i}}}\right)
$$

According to the statement of the mutual synchronization problem, mutual synchronization occurs when the angular position and velocity of all the robots $q_{i}, \dot{q}_{i}$, $i=1, \ldots, p$, coincide at any instant of time, or at least asymptotically, and at the same time coincide with the common desired trajectory $q_{d}, \dot{q}_{d}$. Then one can introduce synchronization indices as

$$
J_{i}\left(q_{i}(t), \dot{q}_{i}(t)\right)=\left[\begin{array}{ll}
q_{i}(t)^{T} & \dot{q}_{i}(t)^{T}
\end{array}\right]
$$

with the corresponding set of functionals

$$
\begin{aligned}
& f_{\text {Sync }}\left(q_{i}, \dot{q}_{i}, q_{j}, \dot{q}_{j}\right)_{i, j}=\left\|J_{i}\left(q_{i}, \dot{q}_{i}\right)-J_{j}\left(q_{j}, \dot{q}_{j}\right)\right\| \quad i, j=1, \ldots, p, j \neq i \\
& f_{\text {Sync }}\left(q_{i}, \dot{q}_{i}, q_{d}, \dot{q}_{d}\right)_{i, i}=\left\|J_{i}\left(q_{i}, \dot{q}_{i}\right)-J_{d}\left(q_{d}, \dot{q}_{d}\right)\right\| \quad i=1, \ldots, p,
\end{aligned}
$$

The chapter is organized as follows. A synchronization controller for frictionless robots, assuming all measurements available is presented in Section 5.2. Section 5.3 presents a modified synchronization controller formed by feedback controllers and nonlinear observers. The stability analysis of the synchronization closed loop system is given in Section 5.3.3. A gain tuning procedure for the observers and feedback controller gains is given in Section 5.4. Section 5.5 describes a friction compensation scheme of the particular friction phenomena modelled by $(5.2,5.3)$. A simulation and experimental study in a one degree of freedom system is presented in Section 5.6. The chapter closes with some concluding remarks and discussion about the proposed synchronization controller, Section 5.7. 


\subsection{Synchronization controller based on state feed- back}

If the full state of all the robots in the multi-composed system is available, then one can propose a mutual synchronization controller as follows.

Let the controller $\tau_{i}$ for the $i$-th robot be given by

$$
\tau_{i}=M_{i}\left(q_{i}\right) \ddot{q}_{r i}+C_{i}\left(q_{i}, \dot{q}_{i}\right) \dot{q}_{r i}+g_{i}\left(q_{i}\right)-K_{d, i} \dot{s}_{i}-K_{p, i} s_{i} \quad i=1, \ldots, p
$$

where $M_{i}\left(q_{i}\right), C_{i}\left(q_{i}, \dot{q}_{i}\right), g_{i}\left(q_{i}\right)$ are defined as in $(5.1), K_{p, i}, K_{d, i} \in \mathbb{R}^{n \times n}$ are positive definite gain matrices, $\dot{s}_{i}$ is the synchronization error at velocity level, $s_{i}$ is the synchronization error at position level.

The synchronization errors $\dot{s}_{i}, s_{i} \in \mathbb{R}^{n}$ are defined as

$$
s_{i}:=q_{i}-q_{r i}, \quad \dot{s}_{i}:=\dot{q}_{i}-\dot{q}_{r i}
$$

with $q_{r i}, \dot{q}_{r i}$ the reference signals.

In order to generate interactions between the robots and to guarantee the synchronous behavior, define the reference signals as

$$
\begin{gathered}
q_{r i}=q_{d}-\sum_{j=1, j \neq i}^{p} K_{c p_{-} i, j}\left(q_{i}-q_{j}\right) \\
\dot{q}_{r i}=\dot{q}_{d}-\sum_{j=1, j \neq i}^{p} K_{c v_{-} i, j}\left(\dot{q}_{i}-\dot{q}_{j}\right), \quad \ddot{q}_{r i}=\ddot{q}_{d}-\sum_{j=1, j \neq i}^{p} K_{c a_{-} i, j}\left(\ddot{q}_{i}-\ddot{q}_{j}\right)
\end{gathered}
$$

where $K_{c p_{-} i, j}, K_{c v_{-} i, j}, K_{c a_{-} i, j} \in \mathbb{R}^{n \times n}, i, j=1, \ldots, p$, are positive semidefinite diagonal matrices that define the interactions - at position, velocity and acceleration levels - between the robots in the system.

Note that the reference signals (5.7) are function of the desired trajectory $q_{d}$ and coupling errors (partial synchronization errors). Thus (5.6) and (5.7) illustrate a trade off in the reference signals. On the one hand each robot will be enforced to follow the desired common trajectory $q_{d}(t)$. On the other hand the robots should mutually synchronize. The second term in the right hand side of $q_{r i}, \dot{q}_{r i}$, and $\ddot{q}_{r i}$ represents the "feedback" of the relative synchronization errors between the $i$-th robot and the other robots in the system. Under appropriate conditions in the controller gains, the reference signals (5.7) give rise to a synchronous behavior between all the robots in the system. The gains $K_{c p_{-} i, j}, K_{c v_{-} i, j}, K_{c a_{-} i, j}$ allow to weigh the synchronization errors between the robots and the desired common trajectory. Therefore, these errors can be penalized; as a result priority to synchronicity between the robots or with respect to the common desired trajectory can be assigned. This is particularly important during transients when large errors can cause instability and/or compromise the synchronous behavior of the complete multi-robot system.

Assumption 5.1 For simplicity it is assumed that for all $i, j=1, \ldots, p$ the coupling gains $K_{c p_{-} i, j}, K_{c v_{-} i, j}, K_{c a \_i, j}$ satisfy

$$
K_{c p_{-} i, j}=K_{c v_{-} i, j}=K_{c a_{-} i, j}=K_{i, j}
$$


Define the partial synchronization errors between the $i$-th and the $j$-th robots in the multi-robot system by

$$
e_{i, j}=q_{i}-q_{j}, \quad \dot{e}_{i, j}=\dot{q}_{i}-\dot{q}_{j}, \quad \text { for all } i, j=1, \ldots, p, \quad i \neq j
$$

and for $j=i$ as

$$
e_{i, i}=q_{i}-q_{d}, \quad \dot{e}_{i, i}=\dot{q}_{i}-\dot{q}_{d}
$$

Then the synchronization errors $\dot{s}_{i}, s_{i} \in \mathbb{R}^{n}$ defined by (5.6) can be written as

$$
s_{i}=e_{i, i}+\sum_{j=1, j \neq i}^{p} K_{i, j} e_{i, j}, \quad \dot{s}_{i}=\dot{e}_{i, i}+\sum_{j=1, j \neq i}^{p} K_{i, j} \dot{e}_{i, j}
$$

note that $s_{i}, \dot{s}_{i}$ are linear combinations of the partial synchronization errors between the robots in the multi-composed system.

Remark 5.2 The norm of the partial synchronization errors $(5.8,5.9)$ correspond to the synchronization functionals (5.4).

\subsubsection{Synchronization closed loop error dynamics}

Substitution of the controller (5.5) and the reference signals (5.7) in the robot dynamics (5.1) yields the synchronization closed loop systems (for $i=1, \ldots, p$ )

$$
\begin{aligned}
& M_{i}\left(q_{i}\right) \ddot{q}_{i}+C_{i}\left(q_{i}, \dot{q}_{i}\right) \dot{q}_{i}=M_{i}\left(q_{i}\right)\left(\ddot{q}_{d}-\sum_{j=1, j \neq i}^{p} K_{i, j}\left(\ddot{q}_{i}-\ddot{q}_{j}\right)\right) \\
& +C_{i}\left(q_{i}, \dot{q}_{i}\right)\left(\dot{q}_{d}-\sum_{j=1, j \neq i}^{p} K_{i, j}\left(\dot{q}_{i}-\dot{q}_{j}\right)\right)-K_{d, i} \dot{s}_{i}-K_{p, i} s_{i}
\end{aligned}
$$

It is obvious that the variable $\ddot{q}_{i}$ generates an algebraic loop, because it appears in both sides of the equality, and thus some problems about the implementation of the controller (5.5) can arise. This algebraic loop will be discussed later, and for the moment we will focus on the stability of the closed loop system.

From the definition of the synchronization errors $s_{i}, \dot{s}_{i}$, given by (5.6), it follows that (5.11) can be written as

$$
M_{i}\left(q_{i}\right) \ddot{s}_{i}=-C_{i}\left(q_{i}, \dot{q}_{i}\right) \dot{s}_{i}-K_{d, i} \dot{s}_{i}-K_{p, i} s_{i} \quad i=1, \ldots, p
$$

Notice that (5.12) implies that the synchronization error dynamics is decoupled for every $i=1, \ldots, p$. In fact all the couplings between the robots are modelled by $s_{i}, \dot{s}_{i}, \ddot{s}_{i}$, such that in these variables the synchronization error dynamics for each robot in the system are decoupled.

\subsubsection{Stability analysis}

The stability properties of the synchronization error dynamics (5.12) are summarized in the following theorem. 
Theorem 5.3 Consider the closed loop system formed by the controller (5.5), the reference signals (5.7) and the robot dynamics (5.1). Then the synchronization errors $s_{i}, \dot{s}_{i}$ are globally asymptotically stable if the control gains $K_{d, i}, K_{p, i}, i=1, \ldots, p$ are positive definite.

Proof: Define the vector $s=\left[\begin{array}{lll}s_{1} & \cdots & s_{p}\end{array}\right]$ and take as Lyapunov function

$$
V(s, \dot{s})=\sum_{i=1}^{p}\left\{\frac{1}{2} \dot{s}_{i}^{T} M_{i}\left(q_{i}\right) \dot{s}_{i}+\frac{1}{2} s_{i}^{T} K_{p, i} s_{i}\right\}
$$

It is obvious that $V(s, \dot{s})$ is positive definite for all $K_{p, i}>0, s_{i}, \dot{s}_{i}$, and $V(s, \dot{s})=0$ if and only if $s_{i}=0, \dot{s}_{i}=0$.

The time derivative of $V(s, \dot{s})$ is given by

$$
\dot{V}(s, \dot{s})=\sum_{i=1}^{p}\left\{\dot{s}_{i}^{T} M_{i}\left(q_{i}\right) \ddot{s}_{i}+\frac{1}{2} \dot{s}_{i}^{T} \dot{M}_{i}\left(q_{i}\right) \dot{s}_{i}+\dot{s}_{i}^{T} K_{p, i} s_{i}\right\}
$$

Along the closed loop error dynamics (5.12) it follows that

$$
\dot{V}(s, \dot{s})=\sum_{i=1}^{p}\left\{-\dot{s}_{i}^{T} K_{d, i} \dot{s}_{i}+\dot{s}_{i}^{T}\left(\frac{1}{2} \dot{M}_{i}\left(q_{i}\right)-C_{i}\left(q_{i}, \dot{q}_{i}\right)\right) \dot{s}_{i}\right\}
$$

By anti-symmetry of the matrix $\frac{1}{2} \dot{M}_{i}\left(q_{i}\right)-C_{i}\left(q_{i}, \dot{q}_{i}\right)$ this reduces to

$$
\dot{V}(s, \dot{s})=-\sum_{i=1}^{p} \dot{s}_{i}^{T} K_{d, i} \dot{s}_{i}
$$

Therefore $\dot{V}(s, \dot{s})$ is negative semi-definite for all $K_{d, i}>0$; thus the synchronization errors $s_{i}, \dot{s}_{i}$ are stable, but asymptotically stability cannot be concluded yet. To prove asymptotic stability Barbalat's lemma is used, see Lemma 2.20.

- $V>0$ and $\dot{V} \leq 0$ imply that $V$ approaches a finite limit, i.e.

$$
\lim _{t \rightarrow \infty} \int_{0}^{t} \dot{V}(s, \dot{s}) d t=\lim _{t \rightarrow \infty}-\sum_{i=1}^{p} \int_{0}^{t} \dot{s}_{i}^{T} K_{d, i} \dot{s}_{i} d t=a
$$

with $a$ a constant.

- From (5.12) it follows that $\ddot{s}_{i}$ is bounded, so that

$$
\ddot{V}(s, \dot{s})=-2 \sum_{i=1}^{p} \dot{s}_{i}^{T} K_{d, i} \ddot{s}_{i}
$$

is bounded. This proves that $\dot{V}(s, \dot{s})$ is uniformly continuous.

- Because of the uniform continuity of $\dot{V}(s, \dot{s}),(5.15)$, and by Barbalat's lemma it follows that

$$
\lim _{t \rightarrow \infty} \dot{V}(s, \dot{s})=0
$$


so, it can be concluded that $\dot{s}_{i}(t) \rightarrow 0$ as $t \rightarrow \infty$.

In the limit when $t \rightarrow \infty$ it follows from (5.12) that

$$
M_{i}\left(q_{i}\right) \ddot{s}_{i}=-K_{p, i} s_{i}
$$

Assume that $s_{i}(t) \neq 0$ as $t \rightarrow \infty$, then it implies that $\ddot{s}_{i}(t) \neq 0$ as

$t \rightarrow \infty$, so that $\dot{s}_{i}(t) \neq 0$ as $t \rightarrow \infty$ which yields a contradiction. Thus the only invariant set that satisfies $\dot{V}(s, \dot{s})=0$ is the origin. Therefore it can be concluded that $s_{i}, \dot{s}_{i}$ are globally asymptotically stable.

It has been proven that the synchronization errors $s_{i}, \dot{s}_{i}$ are globally asymptotically stable. However, note that $s_{i}, \dot{s}_{i}$ are linear combinations of the partial synchronization errors. Therefore, it is still necessary to prove that $s_{i}, \dot{s}_{i}$ being asymptotically stable implies global asymptotic synchronization between the robots.

The following lemma is useful in proving asymptotic stability of the synchronization errors.

Lemma 5.4 Consider the diagonally dominant matrix $M_{c}\left(K_{i, j}\right) \in \mathbb{R}^{(n \cdot p) \times(n \cdot p)}$, given by

$$
M_{c}\left(K_{i, j}\right)=\left[\begin{array}{cccc}
\left(I_{n}+\sum_{j=1, j \neq 1}^{p} K_{1, j}\right) & -K_{1,2} & \cdots & -K_{1, p} \\
-K_{2,1} & \left(I_{n}+\sum_{j=1, j \neq 2}^{p} K_{2, j}\right) & \cdots & -K_{2, p} \\
\vdots & & \ddots & \\
-K_{p, 1} & -K_{p, 2} & \cdots & \left(I_{n}+\sum_{j=1, j \neq p}^{p} K_{p, j}\right)
\end{array}\right]
$$

with $K_{i, j}, i, j=1, \ldots, p$ the coupling matrices defined in the reference trajectories $q_{r i}$ in (5.7), thus $M_{c}\left(K_{i, j}\right)$ can be considered as a coupling matrix between the robots in the multi-composed system.

The matrix $M_{c}\left(K_{i, j}\right)$ is nonsingular for all positive semidefinite diagonal matrices $K_{i, j}, i, j=1, \ldots, p$. Moreover,

$$
M_{c}\left(K_{i, j}\right)\left[\begin{array}{c}
q_{1} \\
q_{2} \\
\vdots \\
q_{p}
\end{array}\right]=\left[\begin{array}{c}
q_{d} \\
q_{d} \\
\vdots \\
q_{d}
\end{array}\right] \Leftrightarrow\left[\begin{array}{c}
q_{1} \\
q_{2} \\
\vdots \\
q_{p}
\end{array}\right]=\left[\begin{array}{c}
q_{d} \\
q_{d} \\
\vdots \\
q_{d}
\end{array}\right]
$$

holds for all positive semidefinite diagonal matrices $K_{i, j}$.

Proof: By definition of the reference signals $q_{r i}$ in (5.7) the coupling gains $K_{i, j}$, $i, j=1, \ldots, p$ are diagonal positive semidefinite matrices, therefore, all their diagonal entries are greater than or equal to zero. By using Gerschgorin's theorem about location of eigenvalues (Stewart and Sun 1990), it follows that all eigenvalues of the matrix $M_{c}\left(K_{i, j}\right) \in \mathbb{R}^{(n \cdot p) \times(n \cdot p)}$ lie in the union of the $n \cdot p$ disks

$$
D_{i, r}: \quad D_{i, r}\left(1+\sum_{j=1, j \neq i}^{p} K_{i, j, r}, \sum_{j=1, j \neq i}^{p}\left|-K_{i, j, r}\right|\right) \quad i=1, \ldots, p, \quad r=1, \ldots, n
$$


where $D(z, w)=\{v \in \mathbb{C}|| v-z \mid \leq w\}$, such that $z$ is the center of the disk, $w$ is the radius of the disk. The subindex $r$ refers to the non-zero entry in the $r$-th row of the matrices $K_{i, j} \in \mathbb{R}^{n \times n}$, i.e. we have a disk for each row of the matrix $M_{c}\left(K_{i, j}\right)$.

Because $K_{i, j} \geq 0$, for all $i, j=1, \ldots, p$, it follows that all the diagonal entries of $K_{i, j}$ are nonnegative, so that for all $i=1, \ldots, p, \quad r=1, \ldots, n$

$$
1+\sum_{j=1, j \neq i}^{p} K_{i, j, r}>\sum_{j=1, j \neq i}^{p} K_{i, j, r}
$$

which implies that zero is not in the union of the disks $D_{i, r}$, thus zero is not an eigenvalue of the matrix $M_{c}\left(K_{i, j}\right)$. Therefore, the matrix $M_{c}\left(K_{i, j}\right)$ is nonsingular for all diagonal positive semidefinite coupling matrices $K_{i, j}, i, j=1, \ldots, p$.

Since the matrix $M_{c}\left(K_{i, j}\right)$ is non singular, it follows that for any $y \in \mathbb{R}^{n \cdot p}$, there is a unique $x \in \mathbb{R}^{n \cdot p}$ such that $M_{c}\left(K_{i, j}\right) x=y$ is satisfied. Consider a vector $x$ with all entries equal to $q_{d}$, then for each row of the product $M_{c}\left(K_{i, j}\right) x$ it follows that

$$
\left(1+\sum_{j=1, j \neq i}^{p} K_{i, j, r}-\sum_{j=1, j \neq i}^{p} K_{i, j, r}\right) q_{d}=q_{d}
$$

Therefore the equality (5.17) holds for all diagonal positive semidefinite matrices $K_{i, j}$.

Theorem 5.5 Global asymptotic stability of the synchronization errors $s_{i}, \dot{s}_{i}$ implies global asymptotic synchronization of all the robots in the multi-composed synchronization system, i.e. for all $i, j=1, \ldots, p$, we have that $q_{i} \rightarrow q_{d}, \dot{q}_{i} \rightarrow \dot{q}_{d}$ as $t \rightarrow \infty$, so that $q_{i} \rightarrow q_{j}, \dot{q}_{i} \rightarrow \dot{q}_{j}$ as $t \rightarrow \infty$.

Proof: From (5.10) it follows that in the limit when $t \rightarrow \infty$

$$
\left[\begin{array}{c}
s_{1} \\
\vdots \\
s_{p}
\end{array}\right]=\left[\begin{array}{c}
e_{1,1}+\sum_{j=1, j \neq 1}^{p} K_{1, j} e_{1, j} \\
\vdots \\
e_{p, p}+\sum_{j=1, j \neq p}^{p} K_{p, j} e_{p, j}
\end{array}\right]=\left[\begin{array}{c}
0 \\
\vdots \\
0
\end{array}\right]
$$

with $r=\frac{1}{2} p(p+1)$ different partial synchronization errors $e_{i, j}$, since by definition $e_{i, j}=-e_{i, j}$.

From the definition of the partial synchronization errors (5.8), (5.9) it follows that (5.18) implies that

$$
M_{c}\left(K_{i, j}\right)\left[\begin{array}{c}
q_{1} \\
q_{2} \\
\vdots \\
q_{p}
\end{array}\right]=\left[\begin{array}{c}
q_{d} \\
q_{d} \\
\vdots \\
q_{d}
\end{array}\right]
$$

with $M_{c}\left(K_{i, j}\right)$ given by $(5.16)$.

Therefore from Lemma 5.4 it follows that

$$
M_{c}\left(K_{i, j}\right)\left[\begin{array}{c}
q_{1} \\
q_{2} \\
\vdots \\
q_{p}
\end{array}\right]=\left[\begin{array}{c}
q_{d} \\
q_{d} \\
\vdots \\
q_{d}
\end{array}\right] \Leftrightarrow\left[\begin{array}{c}
q_{1} \\
q_{2} \\
\vdots \\
q_{p}
\end{array}\right]=\left[\begin{array}{c}
q_{d} \\
q_{d} \\
\vdots \\
q_{d}
\end{array}\right]
$$


The equality (5.19) implies that for all $i, j=1, \ldots, p, q_{i} \rightarrow q_{d}$, , so that $q_{i} \rightarrow q_{j}$. In a similar way it can be proved that $\dot{q}_{i} \rightarrow \dot{q}_{d}$ as $t \rightarrow \infty$, so that $\dot{q}_{i} \rightarrow \dot{q}_{j}$ as $t \rightarrow \infty$.

Remark 5.6 The matrix $M_{c}\left(K_{i, j}\right)$ defined by (5.16) is nonsingular for all positive semidefinite diagonal coupling matrices $K_{i, j}, i, j=1, \ldots, p$, i.e. including $K_{i, j}=0$, see Lemma 5.4. Therefore partial synchronization of the robots in the system can be considered, i.e. some $K_{i, j}=0$. Moreover, by definition of the coupling gains $K_{i, j}$ master slave synchronization can be achieved.

\subsubsection{Algebraic loop}

Substitution of the controller (5.5) and the reference trajectories (5.7) in the robot dynamics (5.1) yields an algebraic loop in the synchronization closed loop systems given by (5.11). The question is how to implement the controller (5.5) and the reference signals (5.7) if they depend on the acceleration $\ddot{q}_{i}(t)$.

By straightforward algebraic manipulation the synchronization closed loop dynamics (5.11) can be rewritten as

$$
M_{c}\left(K_{i, j}\right)\left[\begin{array}{c}
\ddot{q}_{1} \\
\ddot{q}_{2} \\
\vdots \\
\ddot{q}_{p}
\end{array}\right]=\left[\begin{array}{c}
\ddot{q}_{d}-M_{1}\left(q_{1}\right)^{-1}\left(C_{1}\left(q_{1}, \dot{q}_{1}\right) \dot{s}_{1}+K_{d, 1} \dot{s}_{1}+K_{p, 1} s_{1}\right) \\
\ddot{q}_{d}-M_{2}\left(q_{2}\right)^{-1}\left(C_{2}\left(q_{2}, \dot{q}_{2}\right) \dot{s}_{2}+K_{d, 2} \dot{s}_{2}+K_{p, 2} s_{2}\right) \\
\vdots \\
\ddot{q}_{d}-M_{p}\left(q_{p}\right)^{-1}\left(C_{p}\left(q_{p}, \dot{q}_{p}\right) \dot{s}_{p}+K_{d, p} \dot{s}_{p}+K_{p, p} s_{p}\right)
\end{array}\right]
$$

with the matrix $M_{c}\left(K_{i, j}\right)$ given by (5.16).

From Lemma 5.4 it follows that the matrix $M_{c}\left(K_{i, j}\right)$ is always invertible for all positive semidefinite diagonal matrices $K_{i, j}$ for all $i, j=1, \ldots, p$, and therefore

$$
\left[\begin{array}{c}
\ddot{q}_{1} \\
\ddot{q}_{2} \\
\vdots \\
\ddot{q}_{p}
\end{array}\right]=M_{c}\left(K_{i, j}\right)^{-1}\left[\begin{array}{c}
\ddot{q}_{d}-M_{1}\left(q_{1}\right)^{-1}\left(C_{1}\left(q_{1}, \dot{q}_{1}\right) \dot{s}_{1}+K_{d, 1} \dot{s}_{1}+K_{p, 1} s_{1}\right) \\
\ddot{q}_{d}-M_{2}\left(q_{2}\right)^{-1}\left(C_{2}\left(q_{2}, \dot{q}_{2}\right) \dot{s}_{2}+K_{d, 2} \dot{s}_{2}+K_{p, 2} s_{2}\right) \\
\vdots \\
\ddot{q}_{d}-M_{p}\left(q_{p}\right)^{-1}\left(C_{p}\left(q_{p}, \dot{q}_{p}\right) \dot{s}_{p}+K_{d, p} \dot{s}_{p}+K_{p, p} s_{i}\right)
\end{array}\right]
$$

Equation (5.20) represents the synchronization closed loop dynamics (5.11) without any algebraic loop. Therefore (5.20) defines the manifold in which the synchronization closed loop dynamics lie if the controller (5.5) is implemented and there are no uncertainties in the dynamic model (5.1). The synchronization closed loop dynamics written as (5.20) fully represents the closed loop system formed by the robot dynamics (5.1), the controller (5.5) and the reference signals (5.7).

\subsection{Synchronization controller based on estimated variables}

As mentioned in the introduction, it is assumed that only angular joint positions $q_{i}, i=1, \ldots, p$, are measured. Therefore, the synchronization controller (5.5) cannot be implemented. As an option the controller $\tau_{i}$ for the $i$-th robot, given by (5.5), can be modified to only depend on positions measurements $q_{i}$ and $q_{j}$, and estimated values for the velocities $\dot{q}_{i}$ and $\dot{q}_{j}$ and accelerations $\ddot{q}_{i}, \ddot{q}_{j}, i, j=1, \ldots, p, j \neq i$. 
Let the controller $\tau_{i}$ for the $i$-th robot be given by

$$
\tau_{i}=M_{i}\left(q_{i}\right) \widehat{\ddot{q}}_{r i}+C_{i}\left(q_{i}, \widehat{\dot{q}}_{i}\right) \widehat{\dot{q}}_{r i}+g_{i}\left(q_{i}\right)-K_{d, i} \widehat{\dot{s}}_{i}-K_{p, i} s_{i} \quad i=1, \ldots, p
$$

where $M_{i}\left(q_{i}\right), C_{i}\left(q_{i}, \widehat{\dot{q}}_{i}\right), g_{i}\left(q_{i}\right)$ are defined as in (5.1), and $K_{p, i}, K_{d, i} \in \mathbb{R}^{n \times n}$ are positive definite gain matrices. Because of the assumption that only angular joint positions $q_{i}, i=1, \ldots, p$, are measured, the reference signals $\dot{q}_{r i}, \ddot{q}_{r i}$, defined by (5.7), and thus the velocity synchronization error $\dot{s}_{i}$, given by (5.6), cannot be implemented directly. In (5.21) $\widehat{\dot{s}}_{i}$ denotes the estimate of the velocity synchronization error $\dot{s}_{i}$, given by (5.6). $\widehat{\dot{q}}_{i}, \widehat{\ddot{q}}_{r i}$ and $\widehat{\dot{q}}_{r i}$ are estimates of the angular velocity $\dot{q}_{i}$ and the reference signals $\dot{q}_{r i}, \ddot{q}_{r i},(5.7)$.

The estimates $\widehat{\dot{q}}_{r i}, \widehat{\ddot{q}}_{r i}$ are given by

$$
\begin{aligned}
& \widehat{\dot{q}}_{r i}=\dot{q}_{d}-\sum_{j=1, j \neq i}^{p} K_{c v_{-} i, j}\left(\widehat{\dot{q}}_{i}-\widehat{\dot{q}}_{j}\right) \\
& \widehat{\ddot{q}}_{r i}=\ddot{q}_{d}-\sum_{j=1, j \neq i}^{p} K_{c a_{-} i, j}\left(\widehat{\ddot{q}}_{i}-\widehat{\ddot{q}}_{j}\right)
\end{aligned}
$$

where $\widehat{\dot{q}}_{i}, \widehat{\ddot{q}}_{i} \in \mathbb{R}^{n}$ represent estimates of $\dot{q}_{i}, \ddot{q}_{i}$ respectively. Then it follows that the estimate for the synchronization error $\dot{s}_{i}$ is given by

$$
\widehat{\dot{s}}_{i}=\widehat{\dot{q}}_{i}-\widehat{\dot{q}}_{r i}
$$

or in terms of estimates for the partial synchronization errors

$$
\widehat{\dot{s}}_{i}:=\widehat{\dot{e}}_{i, i}+\sum_{j=1, j \neq i}^{p} K_{c v_{-} i, j} \widehat{\dot{e}}_{i, j}
$$

with $\widehat{\dot{e}}_{i, i}, \widehat{\dot{e}}_{i, j}$ given by

$$
\begin{array}{ll}
\hat{\dot{e}}_{i, j}=\widehat{\dot{q}}_{i}-\widehat{\dot{q}}_{j}, & \text { for all } i, j=1, \ldots, p, \quad i \neq j \\
\hat{\dot{e}}_{i, i}=\widehat{\dot{q}}_{i}-\dot{q}_{d}, & \text { for } i=j
\end{array}
$$

For simplicity in the stability analysis, it is assumed that Assumption 5.1 is satisfied, i.e. the positive semidefinite diagonal coupling matrices $K_{c p_{-} i, j}, K_{c v_{-} i, j}, K_{c a_{-} i, j} \in$ $\mathbb{R}^{n \times n}, i, j=1, \ldots, p$, are such that $K_{c p_{-} i, j}=K_{c v_{-} i, j}=K_{c a_{-} i, j}=K_{i, j}$.

\subsubsection{An observer for the joint variables}

An observer for estimating the joint variables $q_{i}, \dot{q}_{i}$ in the dynamic model of the $i$-th robot given by (5.1), is given by

$$
\begin{aligned}
\frac{d}{d t} \widehat{q}_{i} & =\widehat{\dot{q}}_{i}+\mu_{i, 1} \widetilde{q}_{i} \\
\frac{d}{d t} \widehat{\dot{q}}_{i} & =-M_{i}\left(q_{i}\right)^{-1}\left[C_{i}\left(q_{i}, \hat{\dot{q}}_{i}\right) \widehat{\dot{q}}_{i}+g_{i}\left(q_{i}\right)-\tau_{i}\right]+\mu_{i, 2} \widetilde{q}_{i}
\end{aligned}
$$


where $\widehat{q}_{i}, \widehat{\dot{q}}_{i}, \frac{d}{d t} \widehat{\dot{q}}_{i}$ represent estimates for $q_{i}, \dot{q}_{i}, \ddot{q}_{i}$, and the estimation position and velocity errors $\widetilde{q}_{i}$ and $\widetilde{\dot{q}}_{i}, \widetilde{\ddot{q}}_{i}$ are defined by

$$
\widetilde{q}_{i}:=q_{i}-\widehat{q}_{i} \quad \widetilde{\dot{q}}_{i}:=\dot{q}_{i}-\widehat{\dot{q}}_{i}, \quad \widetilde{\ddot{q}}_{i}=\frac{d}{d t}\left(\dot{q}_{i}-\widehat{\dot{q}}_{i}\right)=\frac{d}{d t} \widetilde{\dot{q}}_{i}
$$

and $\mu_{i, 1}, \mu_{i, 2} \in \mathbb{R}^{n \times n}$ are positive definite gain matrices.

The estimated reference signals $\widehat{\ddot{q}}_{r i}(5.23)$ depend on estimates of the accelerations $\widehat{\ddot{q}}_{i}$, $i=1, \ldots, p$. Therefore when $\tau_{i}$, given by (5.21), is substituted in (5.26), it generates an algebraic loop between the set of $p$ observers, in a similar way as in Section 5.2.1. Nevertheless the algebraic loop in the observers (5.26) can be solved by pure algebraic manipulation, such that the observers (5.26), the control $\tau_{i}$ (5.21) and the reference signals $\widehat{\dot{q}}_{r i}, \widehat{\ddot{q}}_{r i}(5.22,5.23)$ can all be implemented.

Lemma 5.7 The observers defined by (5.26) can be written in the form (for $i=$ $1, \ldots, p)$

$$
\begin{aligned}
\frac{d}{d t} \widehat{q}_{i} & =\widehat{\dot{q}}_{i}+\mu_{i, 1} \widetilde{q}_{i} \\
\frac{d}{d t} \widehat{\dot{q}}_{i} & =f\left(\ddot{q}_{d}, q_{j}, \widehat{\dot{q}}_{j}, \widetilde{q}_{j}, e_{j}, \widehat{\dot{e}}_{j}\right) \quad j=1, \ldots, p
\end{aligned}
$$

with $f(\cdot)$ a known nonlinear function that is Lipschitz in $\ddot{q}_{d}, q_{j}, \widehat{\dot{q}}_{j}, \widetilde{q}_{j}, e_{j}, \widehat{\dot{e}}_{j}$.

Proof. After substitution of $\tau_{i}$ (5.21) in the observer defined by (5.26), it follows that the second equation of (5.26) can be written as (for $i=1, \ldots, p$ )

$$
\begin{array}{r}
\left(I+\sum_{j=1, j \neq i}^{p} k_{i, j}\right) \frac{d}{d t} \widehat{\dot{q}}_{i}-\sum_{j=1, j \neq i}^{p} k_{i, j} \frac{d}{d t} \widehat{\dot{q}}_{j}=\ddot{q}_{d}+\mu_{i, 2} \widetilde{q}_{i}+ \\
-M_{i}\left(q_{i}\right)^{-1}\left[C_{i}\left(q_{i}, \widehat{\dot{q}}_{i}\right) \widehat{\dot{s}}_{i}+K_{d, i} \widehat{\dot{s}}_{i}+K_{p, i} s_{i}\right]
\end{array}
$$

such that a system of $n \cdot p$ equations with $n \cdot p$ unknowns is obtained.

Define the vectors $x, y \in \mathbb{R}^{n \cdot p}$ as

$$
\begin{aligned}
& x=\left[\begin{array}{llll}
\frac{d}{d t} \hat{\dot{q}}_{1}^{T} & \frac{d}{d t} \hat{\dot{q}}_{2}^{T} & \cdots & \frac{d}{d t} \hat{\dot{q}}_{p}^{T}
\end{array}\right]^{T} \\
& y=\left[\begin{array}{llll}
y_{1}^{T} & y_{2}^{T} & \cdots & y_{p}^{T}
\end{array}\right]^{T}
\end{aligned}
$$

where

$$
y_{i}=\ddot{q}_{d}+\mu_{i, 2} \widetilde{q}_{i}-M_{i}\left(q_{i}\right)^{-1}\left[C_{i}\left(q_{i}, \widehat{\dot{q}}_{i}\right) \widehat{\dot{s}}_{i}+K_{d, i} \widehat{\dot{s}}_{i}+K_{p, i} s_{i}\right]
$$

then the system of differential equations defined by (5.29) can be written as

$$
M_{c}\left(K_{i, j}\right) x=y
$$

with the matrix $M_{c}\left(K_{i, j}\right)$ given by (5.16).

Since the matrix $M_{c}\left(K_{i, j}\right)$ is non singular, see Lemma 5.4 , then the system of coupled differential equations (5.29), admits a unique solution, given by

$$
x=M_{c}\left(K_{i, j}\right)^{-1} y
$$


Note that the above solution corresponds to the algebraic system of differential equations (5.29), but not to the differential equations, since by definition $x_{i}=\frac{d}{d t} \widehat{\hat{q}}_{i}^{T}$ for $i=1, \ldots, p$.

The observers defined by (5.28) are an equivalent representation of the observers defined by (5.26), but in (5.28) the algebraic loop has been avoided by decoupling the acceleration dependency in (5.26). For implementation purposes the observer (5.28) is more convenient than (5.26), however (5.26) allows a more straightforward stability analysis than (5.28).

Figure 5.2 shows a schematic representation of the proposed synchronization controller for rigid joint robots $(p=2)$.

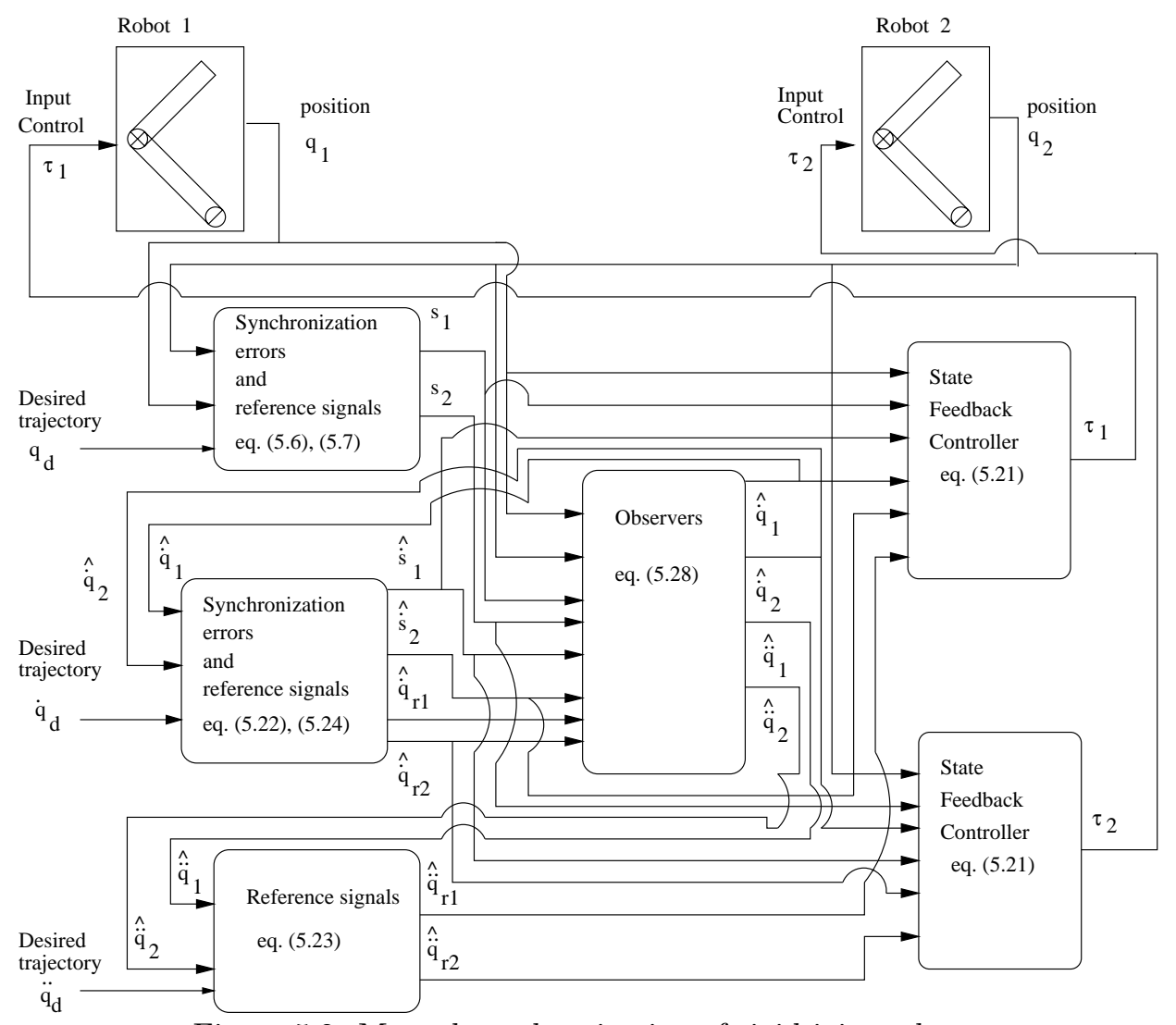

Figure 5.2: Mutual synchronization of rigid joint robots.

\subsubsection{Synchronization closed loop error dynamics}

Throughout the formulation of the error dynamics it is assumed that Assumption 5.1 is satisfied. For simplicity in the closed loop error formulation and the stability analysis the following assumption is introduced.

Assumption 5.8 The gains in the controller (5.21) and the observers (5.26) are a positive multiple of the unit matrix, i.e of the form $K=k I$ with $k$ a positive scalar 
and I the identity matrix of appropriate dimensions. It is also assumed that the gain matrices at velocity and position level are equal for all the observers, i.e.

$$
\mu_{i, 1}=\mu_{1} I, \quad \mu_{i, 2}=\mu_{2} I, \quad \text { for } \quad \text { all } \quad i=1, \ldots, p
$$

Lemma 5.9 The synchronization error dynamics of the closed loop formed by the $p$ robots, modelled by (5.1), the synchronization controllers (5.21), and the observers (5.26), is given by

$$
\ddot{\widetilde{q}}_{i}=M_{i}\left(q_{i}\right)^{-1} C_{i}\left(q_{i}, \dot{\widetilde{q}}_{i}+\mu_{1} \widetilde{q}_{i}\right)\left(\dot{\widetilde{q}}_{i}+\mu_{1} \widetilde{q}_{i}-2 \dot{q}_{d}-2 \dot{e}_{i, i}\right)-\mu_{1} \dot{\widetilde{q}}_{i}-\mu_{2} \widetilde{q}
$$

$$
\begin{aligned}
M_{i}\left(q_{i}\right) \ddot{s}_{i}= & -C_{i}\left(q_{i}, \dot{e}_{i, i}+\dot{q}_{d}\right) \dot{s}_{i}-K_{d, i} \dot{s}_{i}-K_{p, i} s_{i} \\
& -C_{i}\left(q_{i}, \dot{e}_{i, i}+\dot{q}_{d}-\dot{\widetilde{q}}_{i}-\mu_{1} \widetilde{q}_{i}\right) \sum_{j=1, j \neq i}^{p} K_{i, j}\left(\dot{\widetilde{q}}_{i}+\mu_{1} \widetilde{q}_{i}-\dot{\widetilde{q}}_{j}-\mu_{1} \widetilde{q}_{j}\right) \\
& -C_{i}\left(q_{i}, \dot{\widetilde{q}}_{i}+\mu_{1} \widetilde{q}_{i}\right)\left(\dot{q}_{d}-\sum_{j=1, j \neq i}^{p} K_{i, j} \dot{e}_{i, j}\right) \\
& +K_{d, i}\left(\dot{\widetilde{q}}_{i}+\mu_{1} \widetilde{q}_{i}+\sum_{j=1, j \neq i}^{p} K_{i, j}\left(\dot{\tilde{q}}_{i}+\mu_{1} \widetilde{q}_{i}-\dot{\tilde{q}}_{j}-\mu_{1} \widetilde{q}_{j}\right)\right) \\
& +M_{i}\left(q_{i}\right) \sum_{j=1, j \neq i}^{p} K_{i, j}\left(M_{i}\left(q_{i}\right)^{-1} C_{i}\left(q_{i}, \dot{\tilde{q}}_{i}+\mu_{1} \widetilde{q}_{i}\right)\left(\dot{\widetilde{q}}_{i}+\mu_{1} \widetilde{q}_{i}-2 \dot{q}_{d}-2 \dot{e}_{i, i}\right)\right. \\
& -\mu_{1} \dot{\widetilde{q}}_{i}-\mu_{2} \widetilde{q}_{i}+\mu_{1} \dot{\widetilde{q}}_{j}+\mu_{2} \widetilde{q}_{j} \\
& \left.-M_{j}\left(q_{j}\right)^{-1} C_{j}\left(q_{j}, \dot{\widetilde{q}}_{j}+\mu_{1} \widetilde{q}_{j}\right)\left(\dot{\tilde{q}}_{j}+\mu_{1} \widetilde{q}_{j}-2 \dot{q}_{d}-2 \dot{e}_{j, j}\right)\right)
\end{aligned}
$$

Proof: The proof is given in Appendix F.

Note that the second equation of the coupled synchronization error dynamics (5.31) corresponds to the synchronization error (5.12) with a disturbance that vanishes when $\widetilde{q}_{i}=0, \dot{\widetilde{q}}_{i}=0$. Therefore, it can be expected that if the estimation errors $\widetilde{q}_{i}, \dot{\widetilde{q}}_{i}$ tend asymptotically to zero, then the origin of the synchronization errors $s_{i}, \dot{s}_{i}$ is still an equilibrium point for (5.31). Thus, we can conjecture that must exist conditions on the observer gains, control gains and coupling gains that ensure stability (asymptotic stability) of the coupled synchronization errors.

\subsubsection{Stability analysis}

The following assumption is required to prove stability of the synchronization closed loop system.

Assumption 5.10 The common desired trajectory at velocity level $\dot{q}_{d}(t)$ is bounded, i.e. there exist a positive scalar $V_{M}$ such that

$$
\sup _{t}\left\|\dot{q}_{d}(t)\right\|=V_{M}<\infty
$$


Based on the Assumptions 5.1, 5.8, and 5.10, the main result of this chapter is formulated as follows.

Theorem 5.11 Consider a multi-robot system formed by $p$ rigid joint robots with dynamic models given by (5.1). Each robot in closed loop with the controller (5.21), the reference signals (5.22, 5.23) and the observers (5.26). Introduce a positive scalar parameter $\eta_{0}$, which is defined and used throughout the proof.

Then the $p$ robots in the multi-composed system are semiglobally exponentially synchronized, i.e. for $i, j=1, \ldots, p, q_{i} \rightarrow q_{j}, \dot{q}_{i} \rightarrow \dot{q}_{j}$ exponentially in a region that can be made arbitrarily large, if the scalar in the gains $K_{p, i} K_{d, i}, \mu_{1}, \mu_{2}$ are chosen such that for $i=1, \ldots, p$

$$
\begin{gathered}
K_{p, i}>0, \quad K_{d, i}>0, \quad \eta_{0}>0 \\
\mu_{1}>\max \left\{\mu_{1,1}^{*}, \ldots, \mu_{1, p}^{*}\right\} \\
\mu_{2}>\max \left\{\frac{1}{M_{i, m}^{2}}\left(\eta_{0}^{2}-\eta_{0} \mu_{1}-2 V_{M} C_{i, M}\left(\mu_{1}+\eta_{0} M_{i, m}^{-1}\right)\right), \mu_{2,1}^{*}, \ldots, \mu_{2, p}^{*}\right\}
\end{gathered}
$$

where $\mu_{i, 1}^{*}, \mu_{i, 2}^{*}$ are scalars given in the gain tuning procedure in Section 5.4, and $\star_{m}$, $\star_{M}$ stand for the minimum and maximum eigenvalue of the matrix $\star$.

Proof: A sketch of the proof is presented in this section. First the Lyapunov function and conditions for positive definitiveness are presented, then the derivative of the Lyapunov function along the closed loop error dynamics $(5.30,5.31)$ is bounded and sufficient conditions for negative definiteness are formulated. The details behind the proof can be found in Appendix G.

\section{Lyapunov function}

Consider the coupled synchronization error dynamics given by (5.30) and (5.31). Define the vectors $s, \widetilde{q}$ as

$$
s=\left[\begin{array}{lll}
s_{1} & \cdots & s_{p}
\end{array}\right], \quad \widetilde{q}=\left[\begin{array}{lll}
\widetilde{q}_{1} & \cdots & \widetilde{q}_{p}
\end{array}\right]
$$

and take as a Lyapunov function

$$
V(\dot{s}, \dot{\widetilde{q}}, s, \widetilde{q})=\sum_{i=1}^{p} V_{i}\left(\dot{s}_{i}, \dot{\widetilde{q}}_{i}, s_{i}, \widetilde{q}_{i}\right)
$$

where

$$
\begin{gathered}
V_{i}\left(\dot{s}_{i}, \dot{\widetilde{q}}_{i}, s_{i}, \widetilde{q}_{i}\right)=V_{i, 1}\left(\dot{s}_{i}, s_{i}\right)+V_{i, 2}\left(\dot{\widetilde{q}_{i}}, \widetilde{q}_{i}\right) \quad i=1, \ldots, p \\
V_{i, 1}\left(\dot{s}_{i}, s_{i}\right)=\frac{1}{2} \dot{s}_{i}^{T} M_{i}\left(q_{i}\right) \dot{s}_{i}+\frac{1}{2} s_{i}^{T} K_{p, i} s_{i} \\
V_{i, 2}\left(\dot{\widetilde{q}}_{i}, \widetilde{q}_{i}\right)=\frac{1}{2}\left[\begin{array}{ll}
\dot{\widetilde{q}}_{i}^{T} & \widetilde{q}_{i}^{T}
\end{array}\right]\left[\begin{array}{cc}
M_{i}\left(q_{i}\right) & \eta_{i}\left(\widetilde{q}_{i}\right) I_{n} \\
\eta_{i}\left(\widetilde{q}_{i}\right) I_{n} & \mu_{2}+\beta_{i} I_{n}
\end{array}\right]\left[\begin{array}{c}
\dot{\widetilde{q}}_{i} \\
\widetilde{q}_{i}
\end{array}\right]
\end{gathered}
$$

with $\eta_{i}\left(\widetilde{q}_{i}\right)$ defined as

$$
\eta_{i}\left(\widetilde{q}_{i}\right)=\frac{\eta_{0}}{1+\left\|\widetilde{q}_{i}\right\|}
$$


$\beta_{i}$ is given by

$$
\beta_{i}=\eta_{0} \mu_{1}+2 V_{M} C_{i, M}\left(\mu_{1}+\eta_{0} M_{i, m}^{-1}\right)-\mu_{2}\left(1-M_{i, m}\right)
$$

where Assumptions 5.8, 5.10 have been used. The scalars $C_{i, M}, M_{i, m}$ are the bounds of the Coriolis term and the inertia matrix, $\eta_{o}$ is a positive scalar to be determined. Note that $\eta_{i}\left(\widetilde{q}_{i}\right)$ is bounded by

$$
0<\eta_{i}\left(\widetilde{q}_{i}\right)<\eta_{0}
$$

Moreover, it follows that

$$
\dot{\eta}_{i} \dot{\widetilde{q}}_{i}^{T} \widetilde{q}_{i}=-\eta_{i}\left(\frac{\widetilde{q}_{i}^{T} \dot{\tilde{q}}_{i}}{1+\left\|\widetilde{q}_{i}\right\|}\right) \dot{\widetilde{q}}_{i}^{T} \widetilde{q}_{i} \leq \eta_{i}\left\|\dot{\widetilde{q}}_{i}\right\|^{2}
$$

so that

$$
\dot{\eta}_{i} \dot{\widetilde{q}}_{i}^{T} \widetilde{q}_{i} \leq \eta_{0}\left\|\dot{\widetilde{q}}_{i}\right\|^{2}
$$

Notice that $V_{i, 1}\left(\dot{s}_{i}, s_{i}\right)$ in (5.38) corresponds to the Lyapunov function (5.13), which has been considered for the case of synchronization based on full state measurements. $V_{i, 1}\left(\dot{s}_{i}, s_{i}\right)$ is a sum of quadratic positive terms, therefore it is positive definite. On the other hand a sufficient condition for positive definiteness of $V_{i, 2}\left(\dot{\widetilde{q}}_{i}, \widetilde{q}_{i}\right)$ is given by

$$
\mu_{2}>\frac{1}{M_{i, m}^{2}}\left(\eta_{0}^{2}-\left(\eta_{0}+2 V_{M} C_{i, M}\right) \mu_{1}-2 V_{M} C_{i, M} \eta_{0} M_{i, m}^{-1}\right)
$$

with $M_{i, m}$ the minimum eigenvalue of the matrix $M_{i}\left(q_{i}\right)$.

Define the coupled synchronization error $s_{c}$ as

$$
s_{c}=\left[\begin{array}{llll}
\dot{s} & \dot{\widetilde{q}} & s & \widetilde{q}
\end{array}\right]
$$

then the Lyapunov function (5.37) satisfies

$$
P_{m}\left\|s_{c}(t)\right\|^{2} \leq V\left(s_{c}(t)\right) \leq P_{M}\left\|s_{c}(t)\right\|^{2}
$$

for some positive scalar $P_{m}, P_{M}$.

\section{Time derivative of the Lyapunov function}

Consider the vector $\widetilde{q}$ defined by $(5.36)$. Then along the error dynamics $(5.30,5.31)$ the time derivative of the Lyapunov function (5.37) has an upperbound given by, (see Appendix $\mathrm{G}$ for a detailed computation)

$$
\dot{V}_{i}\left(\dot{s}_{i}, \dot{\widetilde{q}}_{i}, s_{i}, \widetilde{q}_{i}\right) \leq-\left[\left\|\dot{s}_{i}\right\| \quad\|\dot{\tilde{q}}\|\|\widetilde{q}\|\right] M_{v i}\left[\left\|\dot{s}_{i}\right\|\|\dot{\tilde{q}}\|\|\widetilde{q}\|\right]^{T}+\left\|\Phi_{3, i}\right\|
$$

with $\left\|\Phi_{3, i}\right\|$ an upperbound of $\Phi_{3, i}$, which is given by

$$
\begin{aligned}
& \left\|\Phi_{3, i}\right\|=\left(2\left\|\dot{s}_{i}\right\|+\|\dot{\vec{q}}\|+\mu_{1}\|\widetilde{q}\|\right)\left(\|\dot{\tilde{q}}\|+\mu_{1}\|\widetilde{q}\|\right) \times \\
& \times\left[C_{i, M}\left(\|\dot{\tilde{q}}\|+\eta_{0} M_{i, m}^{-1}\|\widetilde{q}\|\right)+\left\|\dot{s}_{i}\right\| \sum_{j=1, j \neq i}^{p} K_{i, j}\left(C_{i, M}+M_{i, m} M_{j, m}^{-1} C_{j, M}\right)\right] \\
& +C_{i, M}\left\|\dot{s}_{i}\right\|\left(\|\dot{\tilde{q}}\|+\mu_{1}\|\widetilde{q}\|\right)\left(\left\|\dot{s}_{i}\right\|+2\left(\left\|\dot{s}_{i}\right\|+\|\dot{\tilde{q}}\|+\mu_{1}\|\widetilde{q}\|\right) \sum_{j=1, j \neq i}^{p} K_{i, j}\right)
\end{aligned}
$$


and the matrix $M_{v i}$ given by

$$
\begin{aligned}
& M_{v i}=\left[\begin{array}{ccc}
M_{v i, 11} & M_{v i, 12} & \mu_{1} M_{v i, 12} \\
M_{v i, 12} & M_{v i, 22} & 0 \\
\mu_{1} M_{v i, 12} & 0 & M_{v i, 33}
\end{array}\right] \\
& M_{v i, 11}=K_{d i} \\
& M_{v i, 12}=-\frac{1}{2}\left[K_{d i}-V_{M} C_{i, M}+2 V_{M} \sum_{j=1, j \neq i}^{p} K_{i, j}\left(M_{i, m} M_{j, m}^{-1} C_{j, M}-C_{i, M}\right)\right] \\
& M_{v i, 22}=M_{i, m} \mu_{1}-2 \eta_{0}-\frac{1}{2} M_{i, p M}+2 V_{M} C_{i, M} \\
& M_{v i, 33}=\eta_{0}\left(\mu_{2}+2 \mu_{1} V_{M} C_{i, M} M_{i, M}^{-1}\right)
\end{aligned}
$$

Lemma 5.12 The matrix $M_{v, i}$, given by (5.48) is positive definite if

$$
\begin{gathered}
\eta_{0}>0, \quad K_{d i}>0 \\
\mu_{1, i}^{*}>M_{i, m}^{-1}\left(\frac{M_{v i, 12}^{2}}{M_{v i, 11}}+2 \eta_{0}+\frac{1}{2} M_{i, p M}-2 V_{M} C_{i, M}\right) \\
\mu_{2, i}^{*}>\frac{\mu_{1}^{2} M_{v i, 12}^{2} M_{v i, 22}}{\eta_{0}\left(M_{v i, 11} M_{v i, 22}-M_{v i, 12}^{2}\right)}-2 \mu_{1} V_{M} C_{i, M} M_{i, M}^{-1}
\end{gathered}
$$

Proof: Follows from Sylvester's criterion about positive definiteness of a matrix based on the principal minors, see (Johnson 1990).

In $(5.54,5.55) \mu_{1, i}^{*}, \mu_{2, i}^{*}$, are equivalent to $\mu_{1}, \mu_{2}$, but the notation is used to emphasize that for each matrix $M_{v i}, i=1, \ldots, p$, a different $\mu_{1}, \mu_{2}$ will be obtained, therefore a sufficient condition for all the matrices $M_{v i}, i=1, \ldots, p$ being positive definite is to take $\mu_{1}, \mu_{2}$ as the maximum of $\mu_{1, i}^{*}, \mu_{2, i}^{*}$ that are obtained for each $M_{v i}, i=1, \ldots, p$. Consider the vector $s_{c}$ defined by $(5.44)$, then $\dot{V}_{i}\left(\dot{s}_{i}, \dot{\widetilde{q}}_{i}, s_{i}, \widetilde{q}_{i}\right)$ given by $(5.46)$ results in

$$
\dot{V}_{i}\left(\dot{s}_{i}, \dot{\widetilde{q}}_{i}, s_{i}, \widetilde{q}_{i}\right) \leq\left\|s_{c}\right\|^{2}\left(-M_{v i, m}+\alpha\left\|s_{c}\right\|\right)
$$

where $M_{v i, m}$ is the minimum eigenvalue of the matrix $M_{v i}, i=1, \ldots, p$, so that $M_{v i, m}$ is positive if the conditions $(5.53,5.54$, and 5.55) are satisfied.

The coefficient $\alpha$ is determined by $\left\|\Phi_{3, i}\right\|$ and is given by

$$
\begin{aligned}
& \alpha=C_{i, M}\left(1+\mu_{1}\right)\left(1+2\left(2+\mu_{1}\right) \sum_{j=1, j \neq i}^{p} K_{i, j}\right)+\left(3+\mu_{1}\right)\left(1+\mu_{1}\right) \times \\
& \times\left[C_{i, M}\left(1+\eta_{0} M_{i, m}^{-1}\right)+\sum_{j=1, j \neq i}^{p} K_{i, j}\left(M_{i, m} M_{j, m}^{-1} C_{j, M}+C_{i, M}\right)\right]
\end{aligned}
$$

The minimum eigenvalue of $M_{v i}$, i.e. $M_{v i, m}$, is proportional to the gain $\mu_{2}$, while $\alpha$ is independent of $\mu_{2}$. Therefore by increasing $\mu_{2}$ it can be ensured that

$$
\dot{V}_{i}\left(\dot{s}_{i}, \dot{\widetilde{q}}_{i}, s_{i}, \widetilde{q}_{i}\right) \leq\left\|s_{c}\right\|^{2}\left(-M_{v i, m}+\alpha\left\|s_{c}\right\|\right)<0 \quad i=1, \ldots, p
$$


Thus the matrix $\dot{V}(\dot{s}, \dot{\widetilde{q}}, s, \widetilde{q})$, given by (G.1), is negative definite. Moreover, it follows that there exist a positive scalar $\kappa$, such that $\dot{V}$ has an upperbound given by

$$
\dot{V} \leq-\kappa\left\|s_{c}\right\|^{2} \quad \text { for all } t \geq 0
$$

From the last equation and (5.45), we conclude that there exist some constants $m^{*}, \rho>$ 0 , such that

$$
\left\|s_{c}(t)\right\|^{2} \leq m^{*} e^{-\rho t}\left\|s_{c}(0)\right\|^{2} \quad \text { for all } t \geq 0
$$

and thus by the definition of $s_{c}$ given by (5.44), it follows that the synchronization closed loop errors are semi-globally exponentially stable with convergence region $\beta_{c}$ given by

$$
\beta_{c}=\left\{s_{c} \quad \mid\left\|s_{c}\right\|<\frac{M_{v i, m}}{\alpha} \sqrt{\frac{P_{m}}{P_{M}}}\right\}
$$

Since the synchronization error $s_{c}$ is exponentially stable, it follows that $s$, and $s_{i}$, for $i=1, \ldots, p$, are exponentially stable too. The proof that the partial synchronization errors $e_{i, j}, i, j=1, \ldots, p$ are exponentially stable, and thus the robots in the multicomposed system are semi-globally exponentially synchronized, follows in the same lines as in the proof of Theorem 5.5.

Remark 5.13 The stability analysis of the closed loop system formed by the p robots, with dynamic model (5.1), and the controller (5.21) results in semi-global exponential stability, see Theorem 5.11. While the closed loop formed by the p robots and the controller (5.5) results in global asymptotic stability, see Theorem 5.3. Nonetheless, exponential convergence of the closed loop formed by the $p$ robots and the controller (5.5) can be proved by considering the upper bound of the time derivative of the Lyapunov function (5.13), then the proof follows as in Theorem 5.11.

\subsection{Gain tuning procedure}

The gain tuning procedure to ensure the stability results stated in Theorem 5.11 can be summarized as follows

1. Determine the bounds of the physical parameters $M_{i}\left(q_{i}\right), C_{i}\left(q_{i}, \dot{q}_{i}\right), \dot{M}_{i}\left(q_{i}\right)$.

2. Determine the bound of the common desired trajectory at velocity level $\dot{q}_{d}$, i.e. $V_{M}$.

3. Choose positive semidefinite coupling gains $K_{i, j}$ for $i, j=1, \ldots, p, j \neq i$.

4. Choose the scalars on the gains $K_{p, i}$, and $K_{d, i}$, for $i=1, \ldots, p$, and the auxiliary scalar $\eta_{0}$, to be positive.

5. For $i=1, \ldots, p$ determine the value $\mu_{1, i}^{*}$, that is given by (5.54), and take $\mu_{1}$ as the maximum of all $\mu_{1, i}^{*}$.

6. For $i=1, \ldots, p$ determine the value $\mu_{2, i}^{*}$, that is given by (5.55), and take $\mu_{2}$ as the maximum of all $\mu_{2, i}^{*}$ and

$$
\frac{1}{M_{i, m}^{2}}\left(\eta_{0}^{2}-\eta_{0} \mu_{1}-2 V_{M} C_{i, M}\left(\mu_{1}+\eta_{0} M_{i, m}^{-1}\right)\right)
$$




\subsection{Friction compensation}

In a similar way as for external synchronization of rigid joint robots, see Section 3.5, friction phenomena can be considered in the case of mutual synchronization. Considering the dynamic model of the robot manipulators with friction effects given by (5.2), it follows that the feedback controller $\tau_{i}(5.21)$ can be modified as

$$
\begin{aligned}
\tau_{i f} & =\tau_{i}+f_{i}\left(\widehat{\dot{q}}_{i}\right) \\
& =M_{i}\left(q_{i}\right) \widehat{\ddot{q}}_{r i}+C_{i}\left(q_{i}, \widehat{\dot{q}}_{i}\right) \widehat{\dot{q}}_{r i}+g_{i}\left(q_{i}\right)-K_{d, i} \widehat{\dot{s}}_{i}-K_{p, i} s_{i}+f_{i}\left(\widehat{\dot{q}}_{i}\right)
\end{aligned}
$$

with the friction compensation term $f_{i}\left(\widehat{\dot{q}}_{i}\right)$ given by

$$
f_{i}\left(\widehat{\dot{q}}_{i}\right)=B_{v, i} \widehat{\dot{q}}_{i}+B_{f 1, i}\left(1-\frac{2}{1+e^{2 w_{1, i} \hat{\dot{q}}_{i}}}\right)+B_{f 2, i}\left(1-\frac{2}{1+e^{2 w_{2, i} \hat{\dot{q}}_{i}}}\right)
$$

and $\widehat{\dot{q}}_{i}$ the estimated for the velocity of the $i$-th robot, that is obtained by the observer (5.28).

The stability analysis of the closed loop system formed by the synchronization controller (5.21), the observers (5.28), and the robots in the multi-composed systems, follows in the same way as for frictionless rigid joint robots. As in Section 3.5, the support of the stability analysis is the fact that the friction model (5.3) implies that

$$
\left\|f_{i}\left(\widehat{\dot{q}}_{i}\right)-f_{i}\left(\dot{q}_{i}\right)\right\| \leq B_{v, i M}\left\|\widetilde{\dot{q}}_{i}\right\|+2 B_{f 1, i M}+2 B_{f 2, i M}
$$

with $\widetilde{\dot{q}}_{i}$ the velocity estimation error, and $B_{v, i M}, B_{f 1, i M}, B_{f 2, i M}$ the maximum eigenvalue of the coefficient matrices $B_{v, i}, B_{f 1, i}, B_{f 2, i}$.

Because the friction effects appear as an additive term in the robot dynamics (5.1) and the feedback controller (5.59), it follows that the difference $f_{i}\left(\widehat{\dot{q}}_{i}\right)-f_{i}\left(\dot{q}_{i}\right)$ appears in the synchronization closed loop error. Then by considering the Lyapunov function (5.37) it follows that the bound (5.60) appears in the bound of the derivative of the Lyapunov function (G.4). Then following the same steps as in the frictionless robot case, semi-global exponential stability of the closed loop synchronization error can be concluded.

\subsection{Simulation and experimental study}

The proposed mutual synchronization controller for rigid joint robots $\tau_{i f}$, given by (5.59), has been implemented on a one degree of freedom multi-robot system. The robots correspond to the medium link of two identical transposer robots fabricated by the Centre for Manufacturing Technology (CFT) Philips Laboratory. The experimental setup, the dynamic model and, the estimated parameters for the robots are given in Section 2.3. The common desired trajectory $q_{d}(t)$ for both robots, referred to as robot 1 and 2 , is a harmonic series given by $(2.35)$.

The synchronization controllers $\tau_{1 f}, \tau_{2 f}$ are implemented according to (5.59), with $\tau_{1}, \tau_{2}$ as in (5.21). The friction compensation term $f_{i}\left(\widehat{\dot{q}}_{i}\right)$ is a function of the estimated velocity $\widehat{\dot{q}}_{i}$, that is obtained by the observer $(5.26)$.

From the tuning gain procedure given in Section 5.4, it follows that for the coupling gains $K_{1,2}=K_{2,1}=100$, the scalar $\eta_{0}=1$, and the gains $K_{d, 1}=K_{d, 2}=2$, the 
mutual synchronization system is stable if the gains in the observers (5.26) satisfy $\mu_{1}>65, \mu_{2}>2120$. After a series of experiments in order to decrease the partial synchronization position errors $e_{i, j}$, the gains in the controllers and observers were set as listed in Table 5.1. The initial position of the links and the initial conditions in the observers (5.28) were chosen as in Table 5.2.

\begin{tabular}{|c|c|c|c|c|}
\hline & $K_{p, i}$ & $K_{d, i}$ & $\mu_{1}$ & $\mu_{2}$ \\
\hline Robot 1 & 2500 & 50 & 500 & 5000 \\
\hline Robot 2 & 2500 & 50 & 500 & 5000 \\
\hline
\end{tabular}

Table 5.1: Control gains in robots 1 and 2.

\begin{tabular}{|l|c|c|c|c|}
\hline & $q_{i}(0)[\mathrm{rad}]$ & $\dot{q}_{i}(0)[\mathrm{rad} / \mathrm{s}]$ & $\widehat{q}_{i}(0)[\mathrm{rad}]$ & $\widehat{\dot{q}}_{i}(0)[\mathrm{rad} / \mathrm{s}]$ \\
\hline Robot 1 & 2.26 & 0 & 2.13 & 0 \\
\hline Robot 2 & 2.00 & 0 & 1.98 & 0 \\
\hline
\end{tabular}

Table 5.2: Initial conditions in robots 1 and 2.

First a comparative study between simulated and experimental results is presented. The experimental results closely match the simulated results, which validates the model of the system $(2.33,2.34)$ and the estimated physical parameters listed in Table 2.1. Therefore, the predicted stability and convergence properties of the proposed synchronization controller (5.21 or 5.59) are shown by experimental results rather than by numerical simulations.

\section{Simulation and experimental results}

The dynamic model $(2.33,2.34)$ of robot 1 and 2 were implemented in the standard Simulink distribution for Matlab version 6.0 Release 12 to simulate the synchronization closed loop system. The gains and initial conditions were set as in Tables 5.1 and 5.2. The frequency of the common desired trajectory $q_{d}(t)$, given by $(2.35)$, was set as $\omega=0.4 \mathrm{~Hz}$.

Figure 5.3 shows the robot position trajectories, simulated $q_{1, s}, q_{2, s}$ and measured $q_{1}, q_{2}$. The simulated and measured positions match very well after the transient period has finished. The mismatch during the transient is due to large input torques during the simulations, which exceed the maximum torque of the physical robots, $\left(\tau_{\max }=500[\mathrm{Nm}]\right)$, see Figures 5.4 and 5.5.

The simulated and measured input torques $\tau_{1, s}, \tau_{2, s}$ and $\tau_{1}, \tau_{2}$ are presented in Figures 5.4 and 5.5. Although the controller (5.59) has been implemented in both robots, the input controls $\tau_{1, s}, \tau_{2, s}$ and $\tau_{1}, \tau_{2}$ in Figures 5.4 and 5.5 only correspond to the synchronization controller (5.21), i.e. they do not include the friction compensation term $f_{i}\left(\widehat{\dot{q}}_{i}\right)$ of the controller $(5.59)$. 

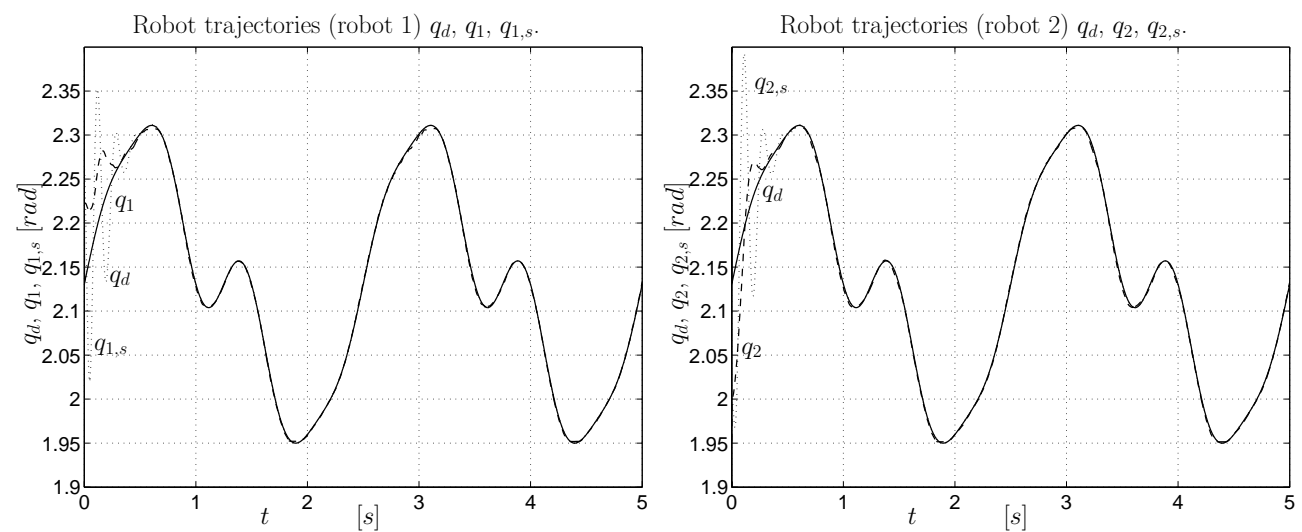

Figure 5.3: Position trajectories: desired $q_{d}$ (solid), experimental $q_{j}$ (dashed), and simulated $q_{j, s}$ (dotted), robot $j=1,2$.
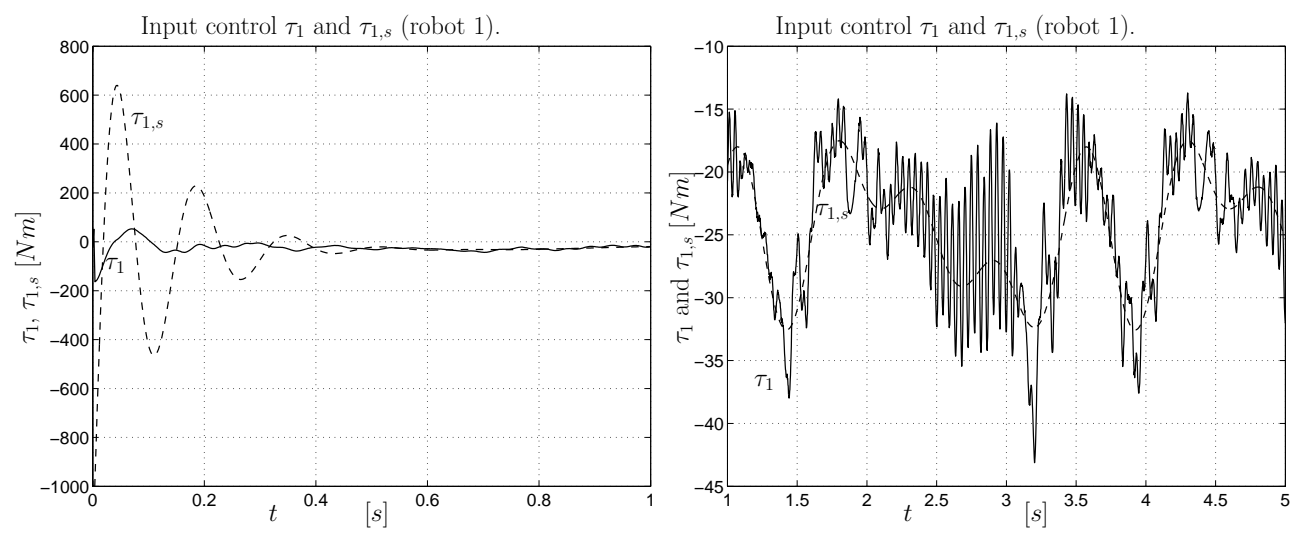

Figure 5.4: Input torques, simulated $\tau_{1, s}$ (dashed) and measured $\tau_{1}$ (solid), robot 1 .
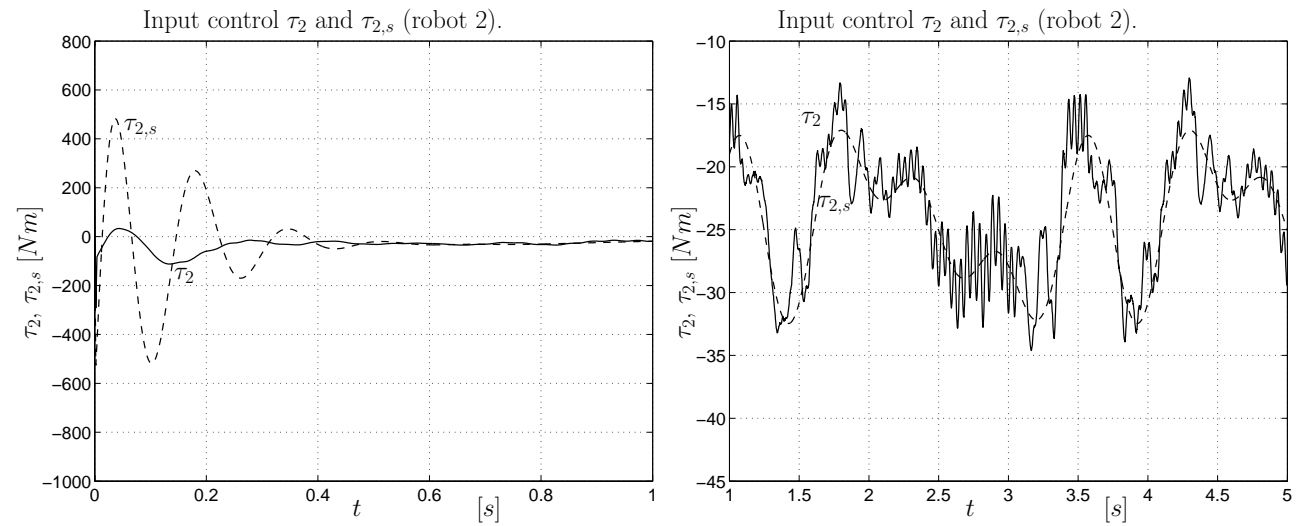

Figure 5.5: Input torques, simulated $\tau_{2, s}$ (dashed) and measured $\tau_{2}$ (solid), robot 2 . 
Since there is no bound on the input control during the simulations, $\tau_{1, s}, \tau_{2, s}$ present large overshoots during the transient. These overshoots are the reason of mismatch between the simulated and measured data during the transient period. After the transient has finished, the simulated and measured slave torques are very similar.

\section{Comparison between synchronization and tracking controllers}

The mutual synchronization controller (5.21) yields a synchronous behavior through the coupling gains $K_{i, j}$. When the coupling gains are set to zero, i.e. $K_{i, j}=0$ the synchronization controller (5.21) becomes the tracking controller proposed by Paden and Panja (1988) but based on estimated velocities. In this section a comparative study between the mutual synchronization controller - coupled case $K_{1,2}=K_{2,1}=100$ - and the tracking controller - uncoupled case $K_{1,2}=K_{2,1}=0$ - is presented. The frequency in the common desired trajectory is $\omega=0.4 \mathrm{~Hz}$.

Figure 5.6 shows the robot position trajectories $q_{d}, q_{1}$ and $q_{2}$ for the coupled and uncoupled cases, while the partial synchronization errors are shown in Figure 5.7. It is obvious that in the coupled case the cross error $e_{1,2}=q_{1}-q_{2}$ converges faster than the errors with respect to $q_{d}$, i.e. $e_{1,1}=q_{1}-q_{d}, e_{2,2}=q_{2}-q_{d}$. This proves the mutual synchronization behavior. Meanwhile for the uncoupled case, the errors $e_{1,1}, e_{2,2}$ converge faster than $e_{1,2}$, since there is not any interaction between them. After the transient has finished the errors in the coupled case are smaller than in the uncoupled one, particularly the cross error $e_{1,2}$. Also notice that the mutual synchronization controller forces a small cross error $e_{1,2}$ by penalizing the errors $e_{1,1}$, $e_{2,2}$, see Figure 5.7(right).

Figure 5.8 presents the estimation error for the joint positions (observer (5.28)) after the transient has finished. Notice that the mutual synchronization controller yields smaller estimation errors than the uncoupled case. The reference signals (5.7) constrain the variety in which the synchronization errors evolve and at the same time give more information to the observer (5.28), such that better convergence in the estimation is achieved.
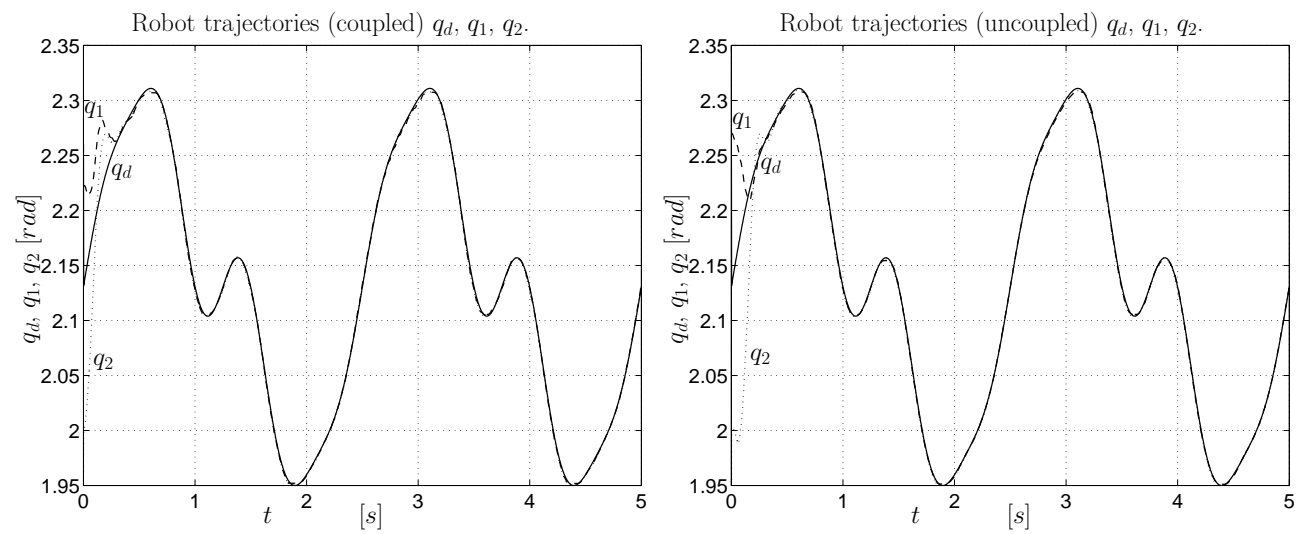

Figure 5.6: Position trajectories: desired $q_{d}$ (solid), $q_{1}$ (dashed), $q_{2}$ (dotted). Coupled case -synchronization- (left) and uncoupled case -tracking- (right). 

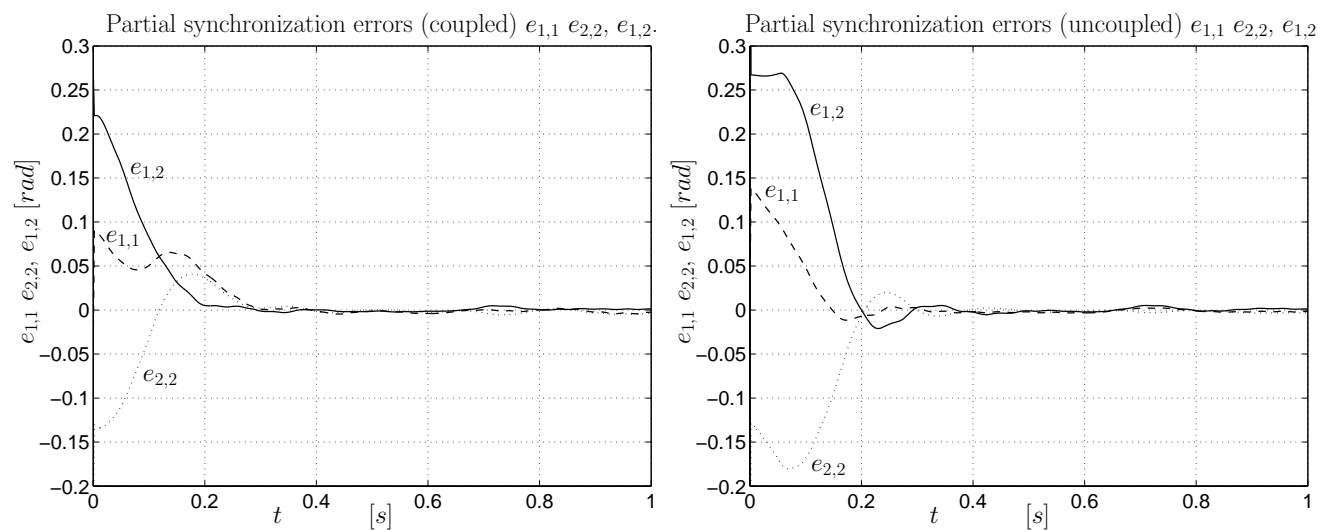

Figure 5.7: Partial synchronization errors: $e_{1,1}$ (dashed), $e_{2,2}$ (dotted), $e_{1,2}$ (solid). Coupled case -synchronization- (left) and uncoupled case -tracking- (right).
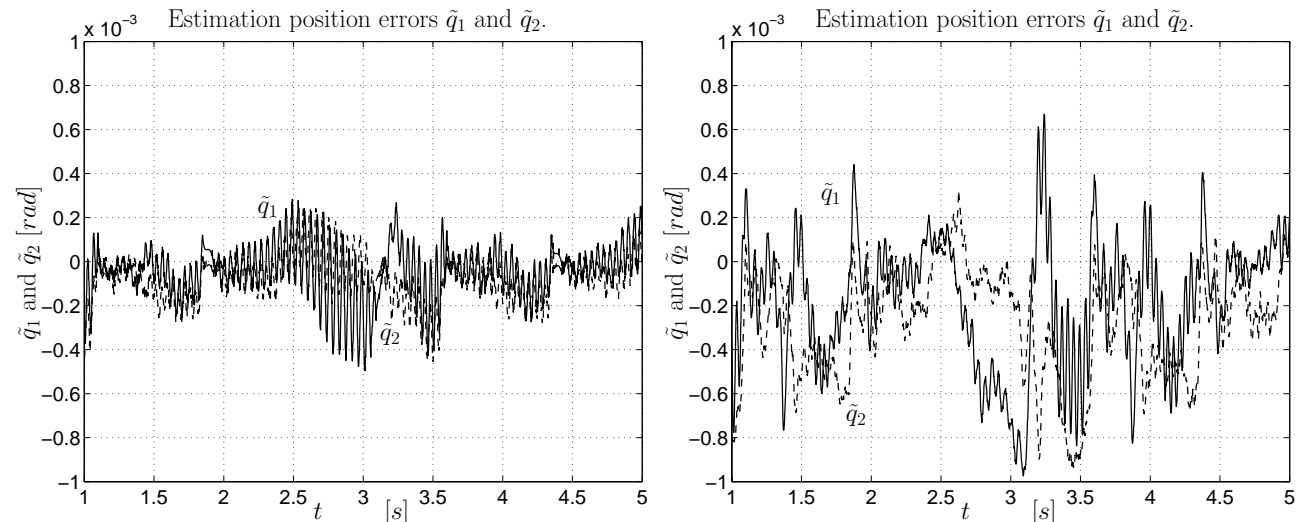

Figure 5.8: Estimation joint position errors: $\widetilde{q}_{1}$ (solid), $\widetilde{q}_{2}$ (dashed). Coupled case -synchronization- (left) and uncoupled case -tracking- (right).

\section{Sensitivity to desired trajectory}

To show the sensitivity of the synchronization controller (5.21) to the desired trajectory $q_{d}(t)$, two different values for the frequency on the common desired trajectory $q_{d}(t)$ have been considered, $\omega=0.2,0.6 \mathrm{~Hz}$. None of the controller gains have been changed during the experiments.

Figure 5.9 show the trajectories $q_{d}, q_{1}, q_{2}$ for $\omega=0.2,0.6 \mathrm{~Hz}$. Note that the mutual synchronization controller yields smaller errors than the external synchronization controller presented in Chapter 3, see Figure 3.8. It is due to the cross synchronization errors introduced by the reference (5.7). 

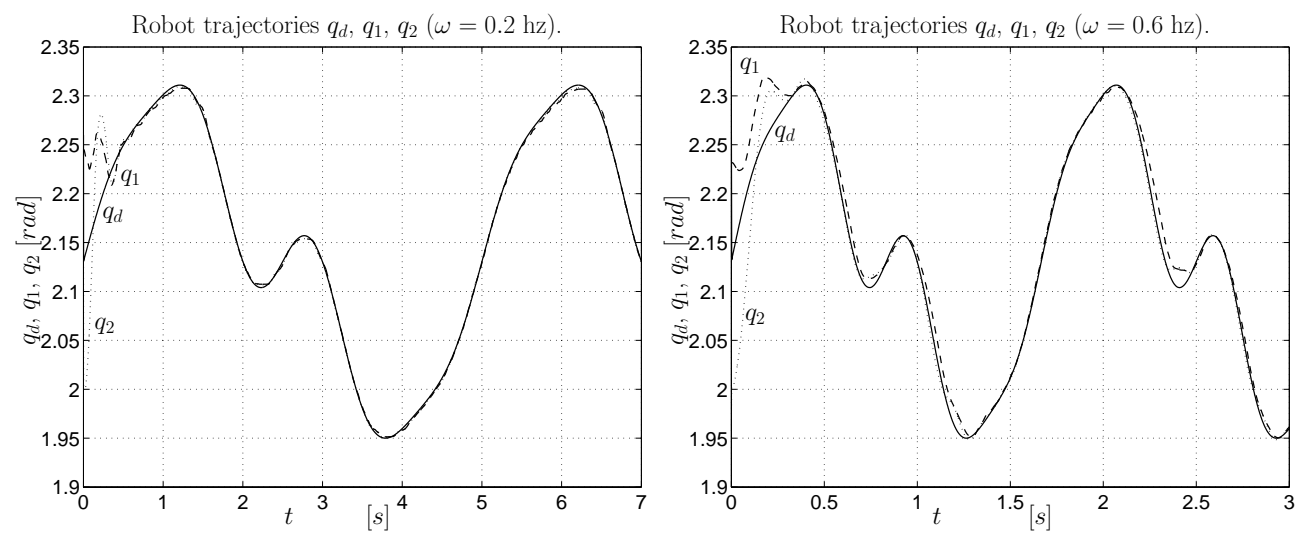

Figure 5.9: Position trajectories: desired $q_{d}$ (solid), $q_{1}$ (dashed) and $q_{2}$ (dotted) for $\omega=0.2 \mathrm{~Hz}, \omega=0.6 \mathrm{~Hz}$.

Although the stability analysis proves that the synchronization errors should converge exponentially to zero independently of the desired trajectory, it is not the case in the Figure 5.9. Smaller synchronization errors are obtained when $\omega=0.2 \mathrm{~Hz}$. The frequency dependency of the convergence in the errors can be due to limited bandwidth of the model $(2.33,2.34)$. Recall that the estimated parameters listed in Section 2.3, Table 2.1 were obtained based on a trajectory with frequency of $\omega=0.4 \mathrm{~Hz}$. Therefore the bandwidth of the dynamic model $(2.33,2.34)$ together with the estimated parameters is center in $0.4 \mathrm{~Hz}$. Thus the synchronization errors for $\omega=0.2,0.6 \mathrm{~Hz}$ are also partially due to the estimated parameters.

\section{Disturbance rejection}

The proposed mutual synchronization controller is robust against disturbances and parametric uncertainty. A payload of $2.0 \mathrm{~kg}$. was used as a disturbance on the robots. The frequency on the common desired trajectory $q_{d}(t)$ was set as $\omega=0.4 \mathrm{~Hz}$.

Figure 5.10(left) shows the partial synchronization errors $e_{1,1}, e_{2,2}, e_{1,2}$ when the payload of $2.0 \mathrm{~kg}$ was set on the robot 1 , from $t=503.8 \mathrm{~s}$ to $t=514.5 \mathrm{~s}$. It can be observed that the load generates a jump in the synchronization errors, nonetheless the robots react to compensate the disturbance and force a small cross error $e_{1,2}$. The partial synchronization error $e_{1,1}$ presents the largest jump because the payload is set on this robot.

Figure 5.10(right) shows the partial synchronization errors $e_{1,1}, e_{2,2}, e_{1,2}$ when the payload of $2.0 \mathrm{~kg}$ was set on the robot 2 , from $t=420.1 \mathrm{~s}$ to $t=428.5 \mathrm{~s}$. Note that the mutual synchronization controller keeps the cross error $e_{1,2}$ small by penalizing the errors $e_{1,1}$ and $e_{2,2}$. Since the payload is set in the robot 2 , this robot presents the largest error with respect to the desired trajectory, i.e. $e_{2,2}$.

The robot 1 seems to be more sensitive to disturbances than the robot 2, although the same controller $(5.21,5.59)$, with the same gains, has been implemented in both robots. The sensitive to disturbances of robot 1 can be due to the differences on the physical parameters of the robots, particularly the friction coefficients. It can be also due to the accuracy of the estimated parameters, see the validation figure presented in Section 2.3. From Figure 2.3 it is evident that the parameters of robot 2 are better estimated than those of robot 1 . Also by simple inspection on the 

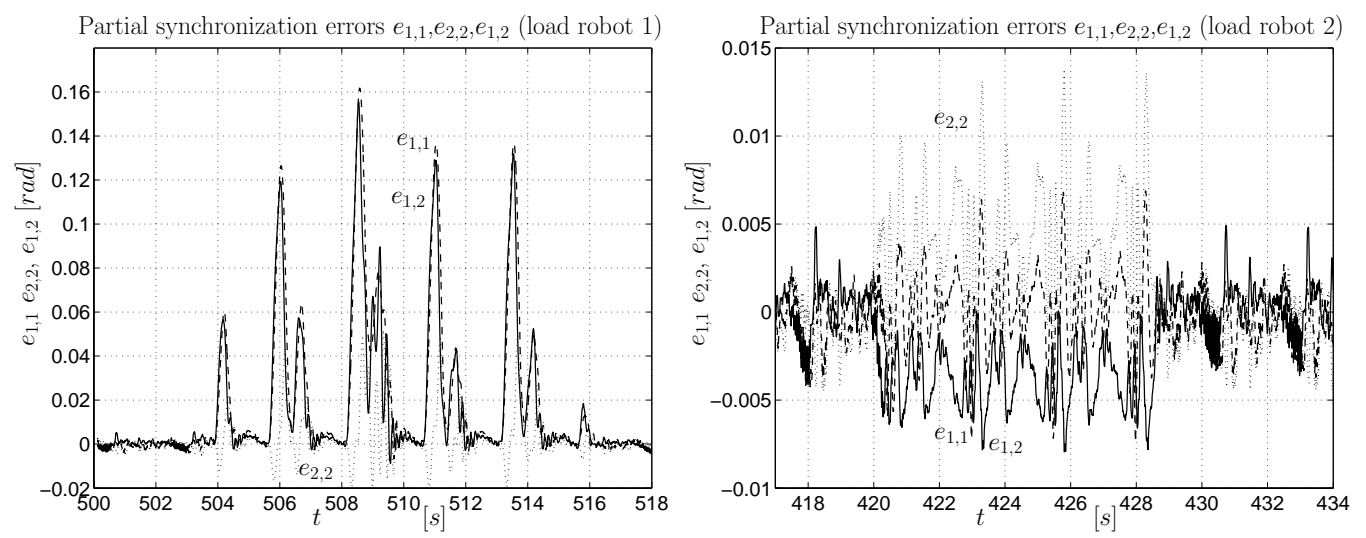

Figure 5.10: Payload of $2.0 \mathrm{Kg}$ on robot 1 and 2, $e_{1,1}$ (dashed), $e_{2,2}$ (dotted), $e_{1,2}$ (solid).

robots it is apparent that the robot 1 presents more weariness on the servomotors and transmission elements than the robot 2 .

Similar sensitivity in the master robot, actually the same robot as robot 1 , has been observed in Figure 3.9 for the external synchronization scheme. However the mutual synchronization controller achieves smaller errors when the payload of $2 \mathrm{Kg}$ is set on robot 1, compare Figures 3.9, 5.10. Similar results follow for robot 2, that corresponds to the slave robot in the external synchronization scheme.

\subsection{Concluding remarks and discussion}

- The proposed synchronizing controller yields practical link position and velocity synchronization in the joint space. Position and velocity synchronization in the Cartesian space, i.e. spatial coordinates, is obtained only if the length of the links of the slave robot are equal to the corresponding links in the robots in the multi-composed system.

- Even without knowledge of the bounds implied in (5.34 - 5.35), the synchronization closed loop system can be made exponentially stable, by selecting the control gains large enough. Although high gains are not desirable since they may amplified the noise in the position measurements and increase the magnitude of the overshoots during transients, diminishing the performance of the synchronization closed loop system.

- The controller and observers (5.21), and (5.26) are model based, nevertheless the stability analysis allows a straightforward robustness analysis for parametric uncertainties. At the same time on-line adaptation of the robot parameters can be included in the proposed synchronization controller by considering linear parametric representation of the dynamic model (5.1), see the work presented by Sun and Mills (2002). Thus adaptive versions of the proposed synchronization controller can be easily obtained.

- Although the result of exponential stability cannot be achieved in practical implementations because of non model dynamics or parametric uncertainties, 
the proposed mutual synchronization controller provides a systematic way of proving stability of the synchronization closed loop system.

- The reference signals (5.7) give a clear insight in the trade off between the synchronization errors between the robots and with respect to the common desired trajectory. These reference signals force the mutual synchronization behavior of the multi-composed system. The mutual synchronization behavior induced by the reference signals (5.7) is particularly useful during transients or sudden disturbances on the robots such as unknown payloads.

- The advantage of the proposed mutual synchronization scheme over traditional tracking controllers and the master slave scheme proposed in Chapter 3 lies in the ability to control the relationships between the position and velocities of all the robots in the system. In other words, the proposed controller regulates not only the convergence of the position and velocity of the robots to the common desired trajectory, but also how these errors converge between them, which greatly improves the performance during transients. Nevertheless, note that the coupling between the robots can generate instability when one of the robots has a major failure, in this situation the couplings from and to the "out of order" robot must be switched to zero.

- The idea of cross coupling errors or partial synchronization errors $e_{i, j}$ has been exploited by several authors, as well as defining synchronization relations that are function of $e_{i, j}$, see for instance (Sun and Mills 2002) and (Feng et al. 1993). However the mentioned works report only set point synchronization, not tracking synchronization. On the other hand the proposed controllers are based on full state feedback, i.e. all states are assumed to be measured.

- The ideas behind the synchronization controller (5.21), the observer (5.26), and the reference signals (5.7) are quite general; thus they can be extended to other systems different from robot manipulators, such as communication systems, mobile robots, coupled generators, multi-satellite systems. 


\section{Chapter 6}

\section{Experimental case study}

\subsection{Introduction}

In Chapters 3 and 5 the convergence and stability properties of the proposed synchronization controllers have been experimentally validated on a one degree of freedom multi-robot system. The multi-robot system corresponds to the medium link of two identical transposer robots fabricated by the Centre for Manufacturing Technology (CFT) Philips Laboratory, see Section 2.3 for a description of the one degree of freedom multi-robot system. However, these transposer robots have 4 degrees of freedom, which are fully actuated by 4 brushless DC servomotors. To support the applicability of the proposed synchronization controllers in complex multi-robot systems this chapter gives synchronization results for the four degrees of freedom of the robots.

Section 6.2 presents a brief description of the transposer robots and the dynamic model of the robots is introduced. Experimental results with the 4 degrees of freedom of the robots with the external synchronization controller (Chapter 3) are presented in Section 6.3. For the mutual synchronization controller (Chapter 5) experimental results with the 4 degrees of freedom are shown in Section 6.4. The chapter closes with some remarks about the implementation of the proposed synchronization controllers, Section 6.5.

\subsection{The CFT transposer robot}

The CFT transposer robot is a Cartesian basic elbow configuration robot. It consists of a two links arm which is placed on a rotating and translational base, and it has a passively actuated tool connected at the end of the outer link, see Figure 6.1. The CFT robot is a pick and place industrial robot used for assembling. It has 4 degrees of freedom in the Cartesian space, denoted by $x_{c i}(i=1, \ldots, 4)$, and 7 degrees of freedom in the joint space, denoted by $q_{j}(j=1, \ldots, 7)$, and is actuated by $4 \mathrm{DC}$ brushless servomotors. Although the robot has 7 degrees of freedom in the joint space, 3 of them are kinematically constrained, with the set of constrained joints given by $\left\{q_{3}, q_{6}, q_{7}\right\}$. Therefore the robot can be represented in the joint space by 4 degrees of freedom $\left\{q_{1}, q_{2}, q_{4}, q_{5}\right\}$ actuated by 4 servomotors. Although the shaft of the motors and the corresponding links are connected by means of belts, the servomotor-link pair proved to be stiff enough to be considered as a rigid joint. 


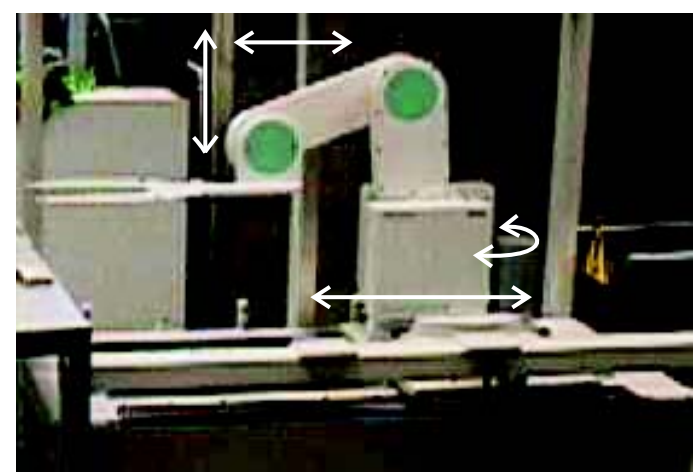

Figure 6.1: The CFT-transposer robot

The 4 Cartesian degrees of freedom are rotation, up and down, forward and backward of the arm, forward and backward of the whole robot, see Figure 6.1. The robot is equipped with encoders attached to the shaft of the motors with a resolution of 2000 PPR, which results in an accuracy of $\pm 0.5[\mathrm{~mm}]$ in all motion directions. The tool connected at the end of the outer link is a kinematically constrained planar support. The tool is passively actuated and designed to remain horizontal at all time. A more detailed description of the structure of the robot can be found in Appendix H.

For implementation of the controllers and communication to the robots, the experimental setup is equipped with a DS1005 dSPACE system, with a processor PPC750, a clock of $480 \mathrm{MHz}$ and a bus clock of $80 \mathrm{MHz}$. Throughout the experiments the sampling frequency of the DS1005 dSPACE system was set to $2 \mathrm{kHz}$.

\subsubsection{Joint space dynamics}

The multi-robot system is formed by two structurally identical transposer robots, so that they have the same kinematic and dynamic model. However, the physical parameters of the robots, such as masses, inertias, friction coefficients are different for both robots. The multi-robot system has been installed in the Dynamics and Control Technology Laboratory of the Department of Mechanical Engineering at the Eindhoven University of Technology.

Hereafter the notation $q_{i}$, for $i=1,2$ refers to the $i$-th robot in the multi-composed system, rather than to the $j$-th joint, $j=1,2,4,5$, in the $i$-th robot. From the Euler-Lagrange approach (Spong and Vidyasagar 1989), (Lewis et al. 1993), and the Denavit-Hartenberg parameters listed in Table H.2, the dynamics of the transposer robots are given by, (see also Section 2.2)

$$
\begin{gathered}
M\left(q_{i}\right) \ddot{q}_{i}+C\left(q_{i}, \dot{q}_{i}\right) \dot{q}_{i}+g\left(q_{i}\right)+f\left(\dot{q}_{i}\right)=\tau_{i}, \quad i=1,2 \\
f\left(\dot{q}_{i}\right)=B_{v, i} \dot{q}_{i}+B_{f 1, i}\left(1-\frac{2}{1+e^{2 w_{1, i} \dot{q}_{i}}}\right)+B_{f 2, i}\left(1-\frac{2}{1+e^{2 w_{2, i} \dot{q}_{i}}}\right)
\end{gathered}
$$

with, $q_{i}=\left[\begin{array}{llll}q_{i, 1} & q_{i, 2} & q_{i, 4} & q_{i, 5}\end{array}\right]^{T}$ the vector of generalized coordinates of robot $i, M\left(q_{i}\right) \in \mathbb{R}^{4 \times 4}$ the symmetric, positive definite inertia matrix, $g\left(q_{i}\right) \in \mathbb{R}^{4}$ denotes the gravity forces, $C\left(q_{i}, \dot{q}_{i}\right) \dot{q}_{i} \in \mathbb{R}^{4}$ represents the Coriolis and centrifugal forces, $f\left(\dot{q}_{i}\right) \in \mathbb{R}^{4}$ are the forces due to friction effects, and $\tau_{i}=\left[\begin{array}{llll}\tau_{i, 1} & \tau_{i, 2} & \tau_{i, 4} & \tau_{i, 5}\end{array}\right]^{T}$ is the vector of external torques. 
The parameters in the matrices $M\left(q_{i}\right), C\left(q_{i}, \dot{q}_{i}\right)$ and the gravity vector $g\left(q_{i}\right)$ are listed in Table H.3 in Appendix H. The physical parameters of the robots in $M\left(q_{i}\right), C\left(q_{i}, \dot{q}_{i}\right)$, $g\left(q_{i}\right)$ and $f\left(\dot{q}_{i}\right)$ have been estimated by using extended Kalman filters and least square methods in a similar way to the work presented in (Kostic et al. 2001).

\subsection{External synchronization of a complex multi- robot system}

Recall that the proposed external synchronization controller for the slave robot $\tau_{s f}$, (3.40), is given by

$$
\tau_{s f}=M_{s}\left(q_{s}\right) \widehat{\ddot{q}}_{m}+C_{s}\left(q_{s}, \widehat{\dot{q}}_{s}\right) \widehat{\dot{q}}_{m}+g_{s}\left(q_{s}\right)-K_{d} \widehat{\dot{e}}-K_{p} e+f_{s}\left(\widehat{\dot{q}}_{s}\right)
$$

with the friction compensation term $f_{s}\left(\widehat{\dot{q}}_{s}\right)$ given by

$$
f_{s}\left(\widehat{\dot{q}}_{s}\right)=B_{v, s} \widehat{\dot{q}}_{s}+B_{f 1, s}\left(1-\frac{2}{1+e^{2 w_{1, s} \hat{\dot{q}}_{s}}}\right)+B_{f 2, s}\left(1-\frac{2}{1+e^{2 w_{2, s} \hat{\dot{q}}_{s}}}\right)
$$

and the estimated variables $\widehat{\dot{e}}, \widehat{\dot{q}}_{s}$ obtained by the observers

$$
\begin{aligned}
\frac{d}{d t} \widehat{e} & =\hat{\dot{e}}+\Lambda_{1} \widetilde{e} \\
\frac{d}{d t} \hat{\dot{e}} & =-M_{s}\left(q_{s}\right)^{-1}\left[C_{s}\left(q_{s}, \hat{\dot{q}}_{s}\right) \hat{\dot{e}}+K_{d} \widehat{\dot{e}}+K_{p} \widehat{e}\right]+\Lambda_{2} \widetilde{e}
\end{aligned}
$$

and

$$
\begin{aligned}
\frac{d}{d t} \widehat{q}_{s} & =\widehat{\dot{q}}_{s}+L_{p 1} \widetilde{q}_{s} \\
\frac{d}{d t} \widehat{\dot{q}}_{s} & =-M_{s}\left(q_{s}\right)^{-1}\left[C_{s}\left(q_{s}, \hat{\dot{q}}_{s}\right) \hat{\dot{e}}+K_{d} \hat{\dot{e}}+K_{p} e\right]+L_{p 2} \widetilde{q}_{s}
\end{aligned}
$$

with the synchronization error $e$ and the estimation errors $\widetilde{e}, \widetilde{q}_{s}$ given by

$$
e=q_{s}-q_{m}, \quad \widetilde{e}=e-\widehat{e}, \quad \widetilde{q}_{s}=q_{s}-\widehat{q}_{s}
$$

The estimated variables $\widehat{\dot{q}}_{m}$ and $\widehat{\ddot{q}}_{m}$ are given by

$$
\widehat{\dot{q}}_{m}=\widehat{\dot{q}}_{s}-\widehat{\dot{e}}, \quad \widehat{\ddot{q}}_{m}=-\left(M_{s}\left(q_{s}\right)^{-1} K_{p}+L_{p 2}\right) \widetilde{e}+L_{p 2} \widetilde{q}_{s}
$$

The experimental setup with the four degrees of freedom of the robots is described in Section 6.2. The dynamic model and the estimated parameters for the robots are given in Section 6.2.1 and Appendix H. The master robot corresponds to robot 1 in Appendix H, while robot 2 has the role of slave robot.

The desired trajectory for the master robot $q_{d}(t)$ is obtained by transformation of a desired trajectory given in Cartesian coordinates $x_{c j, d}(t), j=1, \ldots, 4$, that is given by

$$
\begin{aligned}
x_{c j, d}(t)= & a_{0, j}+a_{1, j} \sin \left(2 s_{f, j} \pi \omega t\right)+a_{2, j} \sin \left(4 s_{f, j} \pi \omega t\right) \\
& +a_{3, j} \sin \left(6 s_{f, j} \pi \omega t\right)+a_{4, j} \sin \left(8 s_{f, j} \pi \omega t\right)
\end{aligned}
$$


with the coefficients $a_{i, j}, i=0, \ldots, 4, j=1, \ldots, 4$ given in Table 6.1. The coefficients $a_{0, j}$ have been chosen as the middle value of the allowed displacements in the robots, while $a_{i, j}, i=1, \ldots, 4$ were chosen to achieve the combination of maximum displacement and velocity allowed by the robots. This is done to generate a trajectory in amplitude that can be executed by the multi-robot system.

\begin{tabular}{|c|c|c|c|c|c|c|}
\hline$a_{i, j}$ & $i=0$ & $i=1$ & $i=2$ & $i=3$ & $i=4$ & $s_{f, j}$ \\
\hline$j=1$ & $-0.1343[\mathrm{~m}]$ & $-0.05[\mathrm{~m}]$ & $-0.015[\mathrm{~m}]$ & $-0.005[\mathrm{~m}]$ & $-0.01[\mathrm{~m}]$ & 1.0 \\
\hline$j=2$ & $0.2766[\mathrm{~m}]$ & $0.05[\mathrm{~m}]$ & $0.03[\mathrm{~m}]$ & $-0.03[\mathrm{~m}]$ & $0.02[\mathrm{~m}]$ & 1.0 \\
\hline$j=3$ & $2.4[\mathrm{rad}]$ & $0.15[\mathrm{rad}]$ & $0.05[\mathrm{rad}]$ & $-0.03[\mathrm{rad}]$ & $0.02[\mathrm{rad}]$ & 1.0 \\
\hline$j=4$ & $-0.265[\mathrm{~m}]$ & $0.2[\mathrm{~m}]$ & $0.1[\mathrm{~m}]$ & $-0.05[\mathrm{~m}]$ & $0.05[\mathrm{~m}]$ & 0.25 \\
\hline
\end{tabular}

Table 6.1: Coefficients of the desired trajectory $x_{c j, d}(t), j=1, \ldots, 4$.

The fundamental frequency of the master robot's desired trajectory $x_{c j, d}(t)$, given by (6.8), is set as $\omega=0.4 \mathrm{~Hz}$.

The joint space desired trajectory $q_{d}(t)$ is obtained by transformation of the desired Cartesian trajectories $x_{c j, d}(t), j=1, \ldots, 4$ using the inverse kinematics, see (H.7).

The master robot is driven by PID controllers with control gains listed as in Table 6.2. After a series of experiments to decrease the synchronization position error $e=q_{s}-q_{m}$, the gains on the slave robot controller (6.3) were set as listed in Table 6.3.

\begin{tabular}{|l|c|c|c|}
\hline & $K_{P}$ & $K_{D}$ & $K_{I}$ \\
\hline joint $q_{1}$ & 11000 & 50 & 2000 \\
\hline joint $q_{2}$ & 10000 & 50 & 1000 \\
\hline joint $q_{4}$ & 40000 & 600 & 1000 \\
\hline joint $q_{5}$ & 40000 & 600 & 1000 \\
\hline
\end{tabular}

Table 6.2: Control gains in the master robot PID controllers.

\begin{tabular}{|l|c|c|c|c|}
\hline & $K_{p}$ & $K_{d}$ & $L_{p 1}$ & $L_{p 2}$ \\
\hline joint $q_{1}$ & 10000 & 1200 & 500 & 100000 \\
\hline joint $q_{2}$ & 8000 & 100 & 500 & 100000 \\
\hline joint $q_{4}$ & 8000 & 100 & 500 & 100000 \\
\hline joint $q_{5}$ & 8000 & 100 & 500 & 100000 \\
\hline
\end{tabular}

Table 6.3: Control gains in the slave robot synchronization controller.

The initial position of the links and the initial conditions in the observers (6.4), (6.5) were chosen as in Table 6.4. The master and slave robot start from a steady state, therefore the joint velocity $\dot{q}(0)$, the estimated joint velocity $\hat{\dot{q}}(0)$, and the estimated synchronization error $\widehat{\dot{e}}(0)$ are all equal to zero. The initial condition for the estimated synchronization error $\widehat{e}(0)$ in observer (6.4) was set equal to zero.

Figures 6.2 and 6.3 show the master $q_{m, j}$ (dashed) and slave $q_{i s, j}$ (solid) position trajectories for the joints $j=1,2,4,5$. The synchronization errors $e=q_{s}-q_{m}$ after the transient period has finished are shown in Figures 6.4 and 6.5.

From Figures 6.2 - 6.5 it is evident that synchronization between the master and slave robot is achieved, such that bounded synchronization errors are obtained. Further experiments showed that the synchronization errors can be decreased by increasing the gains $K_{p}$, which agrees with the result stated in Theorem 3.12 . 


\begin{tabular}{|c|c|c|c|c|}
\hline & $q_{1}(0)[\mathrm{m}]$ & $q_{2}(0)[\mathrm{rad}]$ & $q_{4}(0)[\mathrm{rad}]$ & $q_{5}(0)[\mathrm{rad}]$ \\
\hline master robot & -0.095 & -0.4 & -0.9615 & 2.1473 \\
\hline slave robot & -0.079 & 0.0 & -1.0355 & 2.1165 \\
\hline & $\widehat{q}_{1}(0)[\mathrm{m}]$ & $\widehat{q}_{2}(0)[\mathrm{rad}]$ & $\widehat{q}_{4}(0)[\mathrm{rad}]$ & $\widehat{q}_{5}(0)[\mathrm{rad}]$ \\
\hline slave robot & -0.07 & 0.1 & -1.0 & 2.0 \\
\hline
\end{tabular}

Table 6.4: Initial conditions for master and slave robot.

The observers (6.4) and (6.5) perform very well and yield estimation errors of the order of $1 / 10$ of the synchronization errors $e$. The plots are not shown since they are very similar to the plots presented in Chapter 3, Figure 3.6. Those interested in the estimation errors and performance of the observers (6.4) and (6.5) can find the plots in the website http://www.wtb.tue.nl/ at the Dynamics and Control Group section. The influence of the gains $K_{d}$ and $L_{p 1}$ is related to the response to sudden changes on the system (transients, changes on trajectory, etc.). The increasing of the gains $K_{d}$ and $L_{p 1}$ might result in large overshoots, amplification of noise in the measurements and saturation of the torques in the servo-motors.
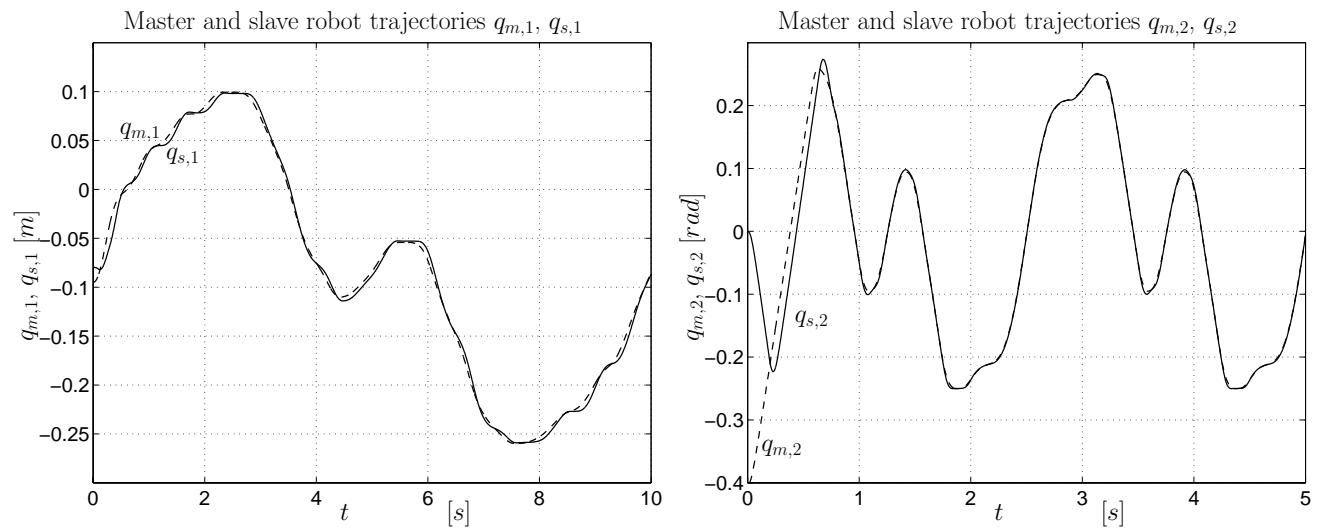

Figure 6.2: Master $q_{m}$ (dashed) and slave $q_{s}$ (solid) positions, joints $q_{1}, q_{2}$
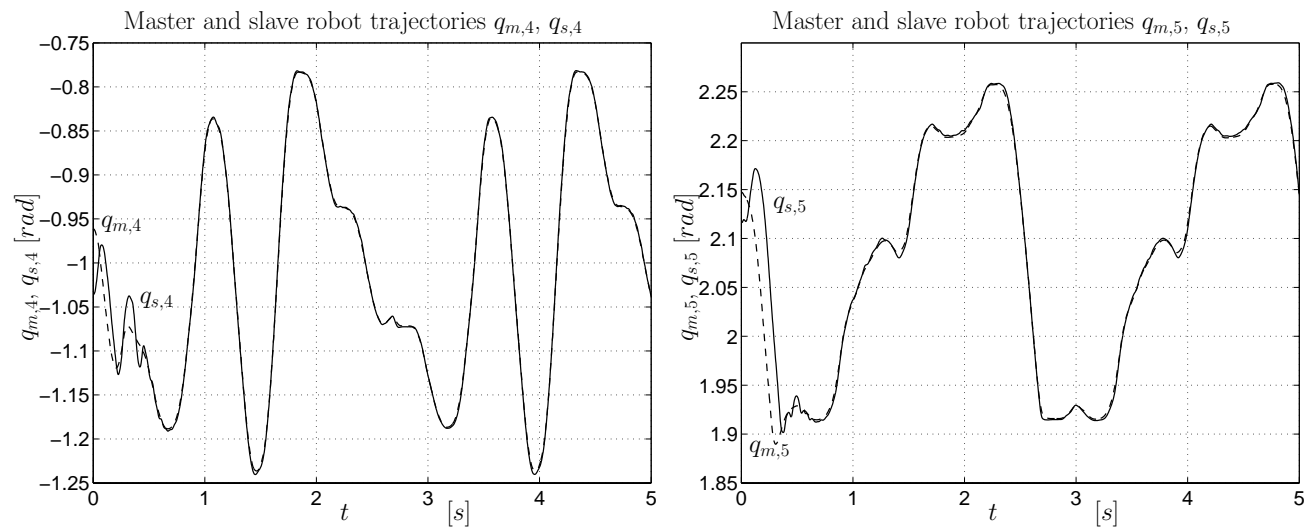

Figure 6.3: Master $q_{m}$ (dashed) and slave $q_{s}$ (solid) positions, joints $q_{4}, q_{5}$ 

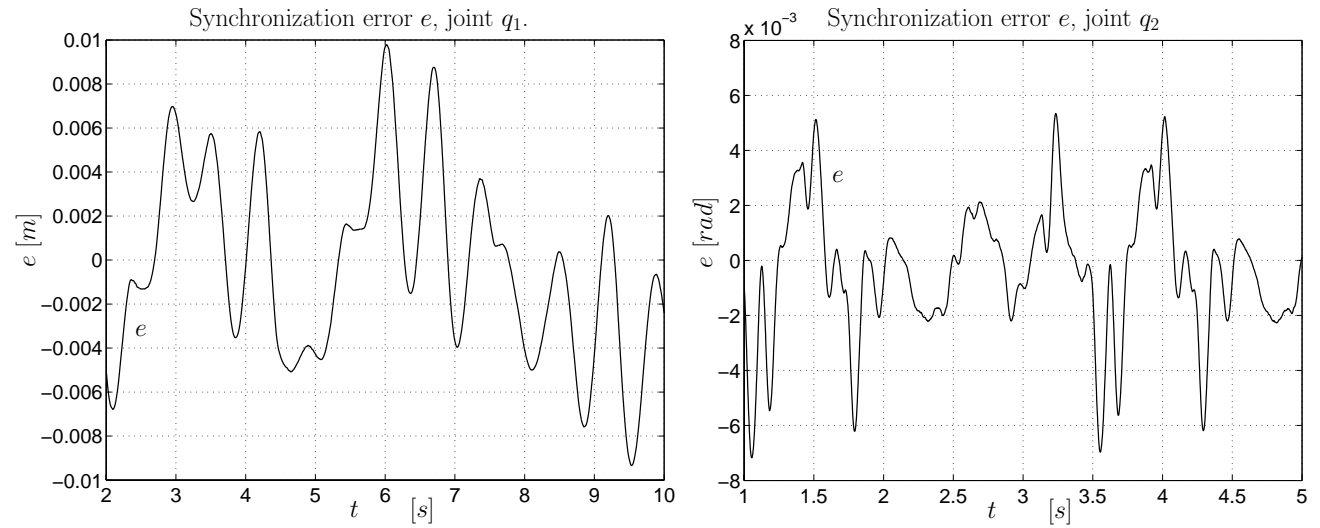

Figure 6.4: Synchronization errors $e$, joints $q_{1}, q_{2}$.
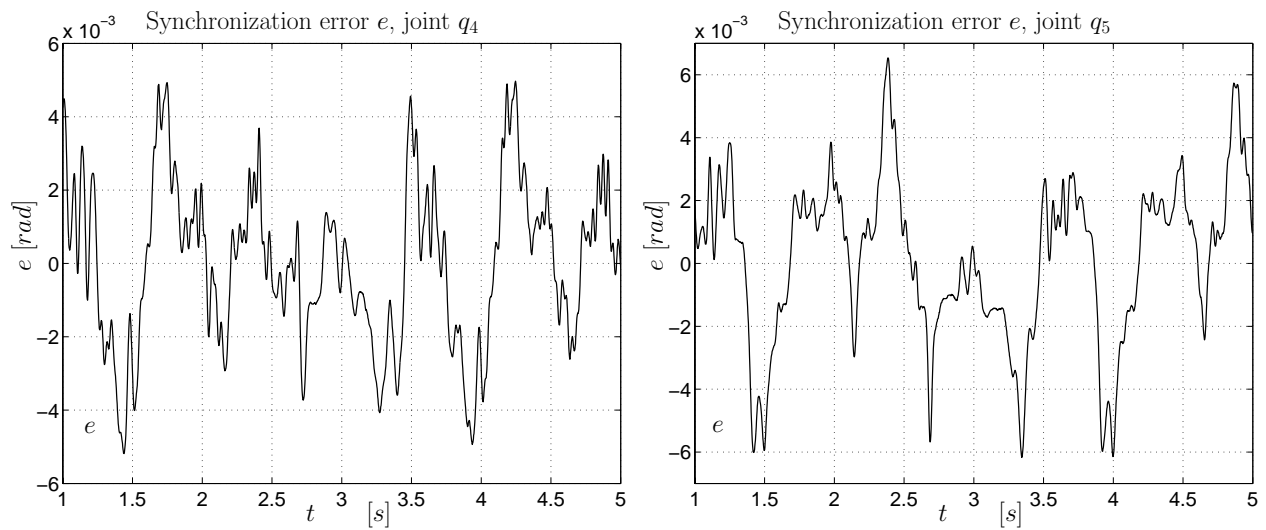

Figure 6.5: Synchronization errors $e$, joints $q_{4}, q_{5}$.

\subsection{Mutual synchronization of a complex multi- robot system}

Recall that the mutual synchronization controller $\tau_{i, f}$, proposed in Chapter $5,(5.59)$, is given by

$$
\tau_{i f}=M_{i}\left(q_{i}\right) \widehat{\ddot{q}}_{r i}+C_{i}\left(q_{i}, \widehat{\dot{q}}_{i}\right) \widehat{\dot{q}}_{r i}+g_{i}\left(q_{i}\right)-K_{d, i} \widehat{\dot{s}}_{i}-K_{p, i} s_{i}+f_{i}\left(\widehat{\dot{q}}_{i}\right)
$$

with the friction compensation term $f_{i}\left(\widehat{\dot{q}}_{i}\right)$ given by

$$
f_{i}\left(\widehat{\dot{q}}_{i}\right)=B_{v, i} \widehat{\dot{q}}_{i}+B_{f 1, i}\left(1-\frac{2}{1+e^{2 w_{1, i} \hat{\dot{q}}_{i}}}\right)+B_{f 2, i}\left(1-\frac{2}{1+e^{2 w_{2, i} \hat{q}_{i}}}\right)
$$

the estimated variables $\widehat{\dot{q}}_{i}$ are obtained by the observer (5.26), i.e.

$$
\begin{aligned}
\frac{d}{d t} \widehat{q}_{i} & =\widehat{\dot{q}}_{i}+\mu_{i, 1} \widetilde{q}_{i} \\
\frac{d}{d t} \widehat{\dot{q}}_{i} & =-M_{i}\left(q_{i}\right)^{-1}\left[C_{i}\left(q_{i}, \widehat{\dot{q}}_{i}\right) \widehat{\dot{q}}_{i}+g_{i}\left(q_{i}\right)-\tau_{i}\right]+\mu_{i, 2} \widetilde{q}_{i}
\end{aligned}
$$


but rewritten such that the algebraic loop is avoided, see Lemma 5.7.

The synchronization $s_{i}, e_{i, j}$, and the estimation $\widetilde{q}_{i}$ errors, $i, j=1,2$, are given by

$$
s_{i}=e_{i, i}+\sum_{j=1, j \neq i}^{p} K_{i, j} e_{i, j}, \quad e_{i, i}=q_{i}-q_{d}, \quad e_{i, j}=q_{i}-q_{j}, \quad \widetilde{q}_{i}=q_{i}-\widehat{q}_{i}
$$

The robots in the multi-robot system are identified as robot 1 (R1) and robot 2 (R2) as in Appendix H.

The desired common trajectory for the robots $q_{d}(t)$ is obtained by transformation of a desired trajectory given in Cartesian coordinates $x_{c j, d}(t), j=1, \ldots, 4$, that is given by (6.8) with the coefficients $a_{i, j}, i=0, \ldots, 4, j=1, \ldots, 4$ given in Table 6.5.

Note that the desired trajectory for mutual synchronization and for external synchronization - Section 6.3, Table 6.1 - are defined by the same signal (6.8), but with different coefficients. This is done to generate a trajectory in amplitude that can be executed by the multi-robot system. The desired trajectory (6.8) with coefficients as in Table 6.1 demands torques that exceed the limits in the servomotors when mutual synchronization is intended. In general the torque to achieve the synchronization goal in the mutual synchronization case is higher than for external synchronization.

\begin{tabular}{|c|c|c|c|c|c|c|}
\hline$a_{i, j}$ & $i=0$ & $i=1$ & $i=2$ & $i=3$ & $i=4$ & $s_{f, j}$ \\
\hline$j=1$ & $-0.1343[\mathrm{~m}]$ & $-0.02[\mathrm{~m}]$ & $-0.015[\mathrm{~m}]$ & $-0.005[\mathrm{~m}]$ & $-0.005[\mathrm{~m}]$ & 1.0 \\
\hline$j=2$ & $0.2766[\mathrm{~m}]$ & $0.05[\mathrm{~m}]$ & $0.03[\mathrm{~m}]$ & $-0.03[\mathrm{~m}]$ & $0.02[\mathrm{~m}]$ & 1.0 \\
\hline$j=3$ & $2.4[\mathrm{rad}]$ & $0.2[\mathrm{rad}]$ & $0.1[\mathrm{rad}]$ & $-0.05[\mathrm{rad}]$ & $0.05[\mathrm{rad}]$ & 1.0 \\
\hline$j=4$ & $-0.265[\mathrm{~m}]$ & $0.15[\mathrm{~m}]$ & $0.05[\mathrm{~m}]$ & $-0.03[\mathrm{~m}]$ & $0.02[\mathrm{~m}]$ & 0.25 \\
\hline
\end{tabular}

Table 6.5: Coefficients of the desired trajectory $x_{c j, d}(t), j=1, \ldots, 4$.

The fundamental frequency of the desired common trajectory $x_{c j, d}(t)$ given by (6.8) is set as $\omega=0.4 \mathrm{~Hz}$. The joint space desired trajectory $q_{d}(t)$ is obtained by transformation of the desired Cartesian trajectories $x_{c j, d}(t), j=1, \ldots, 4$ by considering the inverse kinematics, see (H.7).

The controller and observer gains for the robots R1 and R2 are set equal for the corresponding joints. For the coupling gains symmetric synchronization is chosen, i.e. the coupling gains between the robot are equal, $K_{1,2}=K_{2,1}$. After a series of experiments in order to decrease the synchronization position errors $e_{i, i}$ and $e_{i, j}$, $i, j=1,2$, the gains on the synchronization controller (6.9) and the coupling gains $K_{i, j}$ were set as listed in Table 6.6.

\begin{tabular}{|l|c|c|c|c|c|}
\hline$i=1,2$ & $K_{p, i}$ & $K_{d, i}$ & $\mu_{i, 1}$ & $\mu_{i, 2}$ & $K_{1,2}$ \\
\hline joint $q_{1}$ & 10000 & 600 & 500 & 20000 & 1 \\
\hline joint $q_{2}$ & 10000 & 300 & 600 & 55000 & 2 \\
\hline joint $q_{4}$ & 10000 & 300 & 600 & 55000 & 1 \\
\hline joint $q_{5}$ & 10000 & 300 & 600 & 55000 & 2 \\
\hline
\end{tabular}

Table 6.6: Control and coupling gains in the mutual synchronization controller.

The initial position of the links and the initial conditions in the observers (6.10) were chosen as in Table 6.7. The robots start from a steady state, therefore the joint velocity $\dot{q}_{i}(0)$ and the estimated joint velocity $\widehat{\dot{q}}_{i}(0)$ are all equal to zero. 


\begin{tabular}{|c|c|c|c|c|}
\hline & $q_{1}(0)[\mathrm{m}]$ & $q_{2}(0)[\mathrm{rad}]$ & $q_{4}(0)[\mathrm{rad}]$ & $q_{5}(0)[\mathrm{rad}]$ \\
\hline robot 1 & -0.06 & -0.1 & -1.0 & 2.115 \\
\hline robot 2 & -0.079 & 0.2 & -1.09 & 2.06 \\
\hline & $\widehat{q}_{1}(0)[\mathrm{m}]$ & $\widehat{q}_{2}(0)[\mathrm{rad}]$ & $\widehat{q}_{4}(0)[\mathrm{rad}]$ & $\widehat{q}_{5}(0)[\mathrm{rad}]$ \\
\hline robot 1 and 2 & -0.08 & 0.0 & -0.9899 & 1.9805 \\
\hline
\end{tabular}

Table 6.7: Initial conditions for robots 1 and 2 and observer (6.10).

Figures 6.6 and 6.7 show the desired common trajectory $q_{d}(t)$ (solid), and the position trajectories $q_{i, j}$ for the robots $i=1,2$, (R1 - dashed, R2 - dotted), and the joints $j=1,2,4,5$. The synchronization errors $e_{1,1}=q_{1}-q_{d}$ (dashed), $e_{2,2}=q_{1}-q_{d}$ (dotted), and $e_{1,2}=q_{1}-q_{2}$ (solid), after the transient period has finished are shown in Figures 6.8 and 6.9 .

From Figures $6.6-6.9$ it is evident that synchronization between the robots is achieved, such that bounded synchronization errors are obtained. Particularly Figures 6.7 (right) and 6.9 (right) show that the synchronization errors $e_{1,1}, e_{2,2}$ are penalized in order to minimize the coupled synchronization error $e_{1,2}$. Thus mutual synchronization between the robots is favored over the synchronization between the robots and the desired common trajectory $q_{d}$. This behavior is induced by the coupling gains $K_{i, j}$ through the nominal reference variables $q_{r i}$. Further experiments show that the synchronization errors can be decreased by increasing the gains $K_{p, i}$, which agrees with the stability analysis.

As in the experimental results reported in Chapter 5, Figure 5.8, the estimation errors $\widetilde{q}_{i}$ are at most of the order of $1 / 10$ of the partial synchronization error $e_{1,2}$. Plots of the estimation errors are not presented since they are very similar to Figure 5.8(left) presented in Chapter 5. Those interested in the estimation errors and performance of the observer (6.10) can find the plots in the website http://www.wtb.tue.nl/ at the Dynamics and Control Group section.
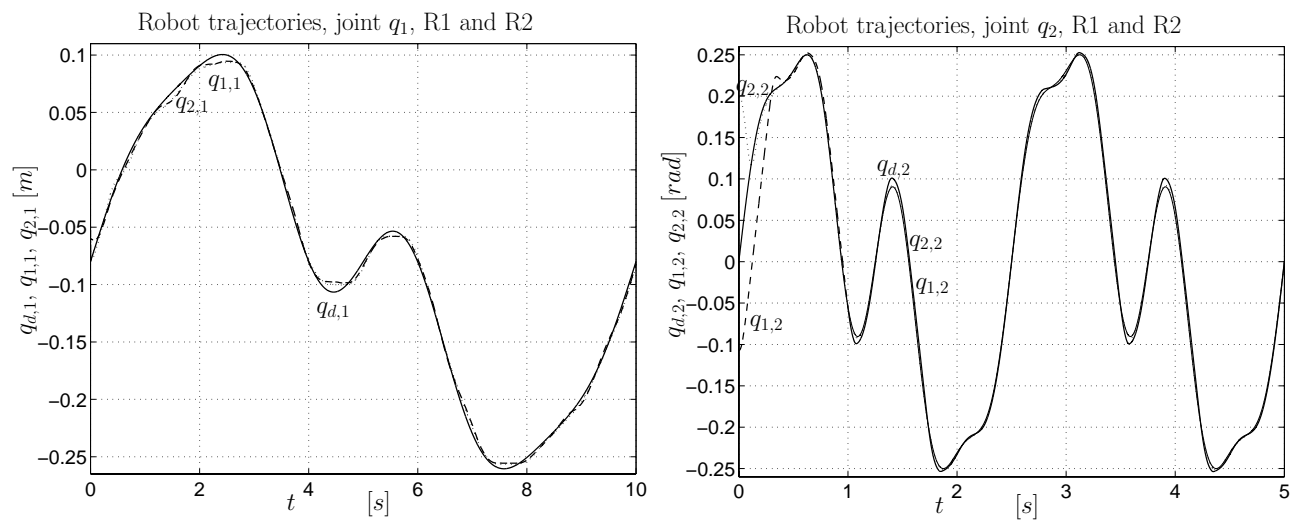

Figure 6.6: Position trajectories: desired $q_{d, j}$ (solid), $q_{1, j}$ (dashed), $q_{2, j}$ (dotted), joints $j=1,2$. 

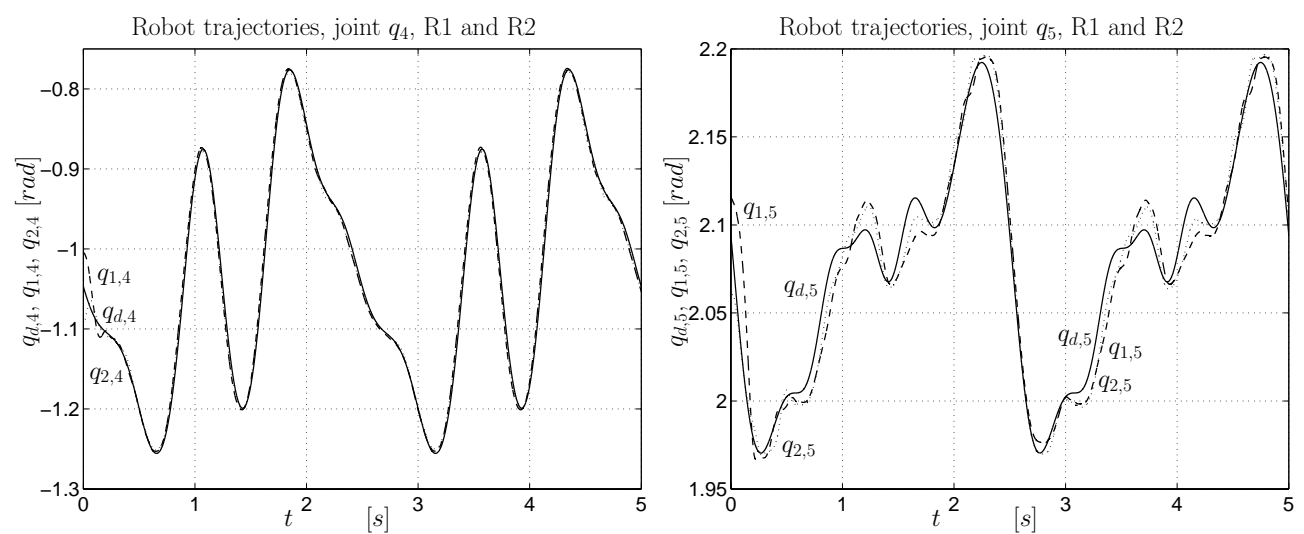

Figure 6.7: Position trajectories: desired $q_{d, j}$ (solid), $q_{1, j}$ (dashed), $q_{2, j}$ (dotted), joints $j=4,5$.
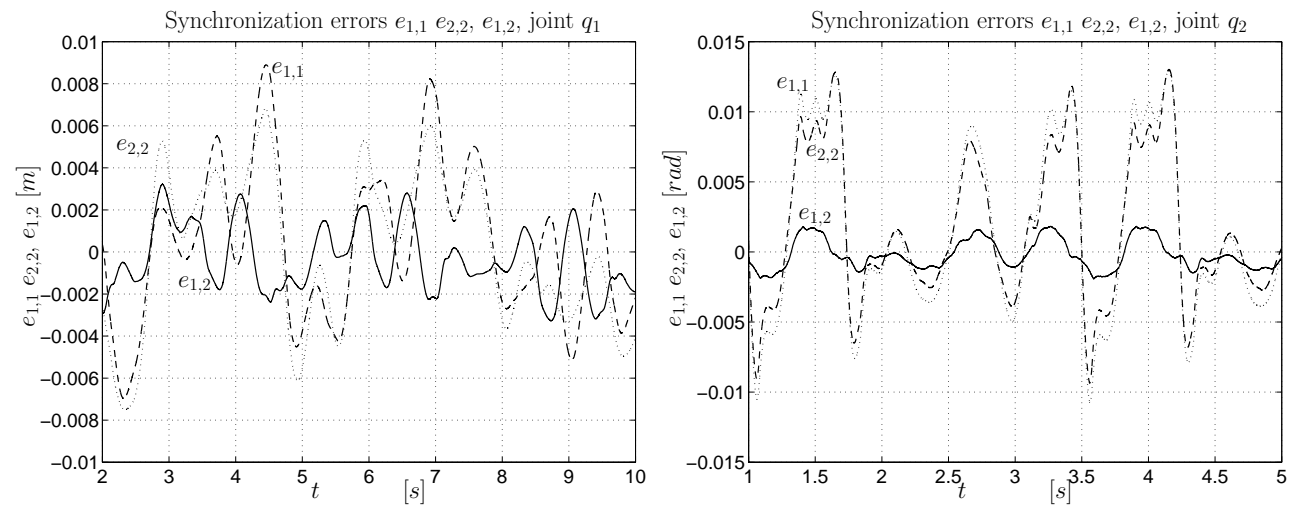

Figure 6.8: Synchronization errors $e_{1,1}$ (dashed), $e_{2,2}$ (dotted), and $e_{1,2}$ (solid), joints $q_{1}, q_{2}$.
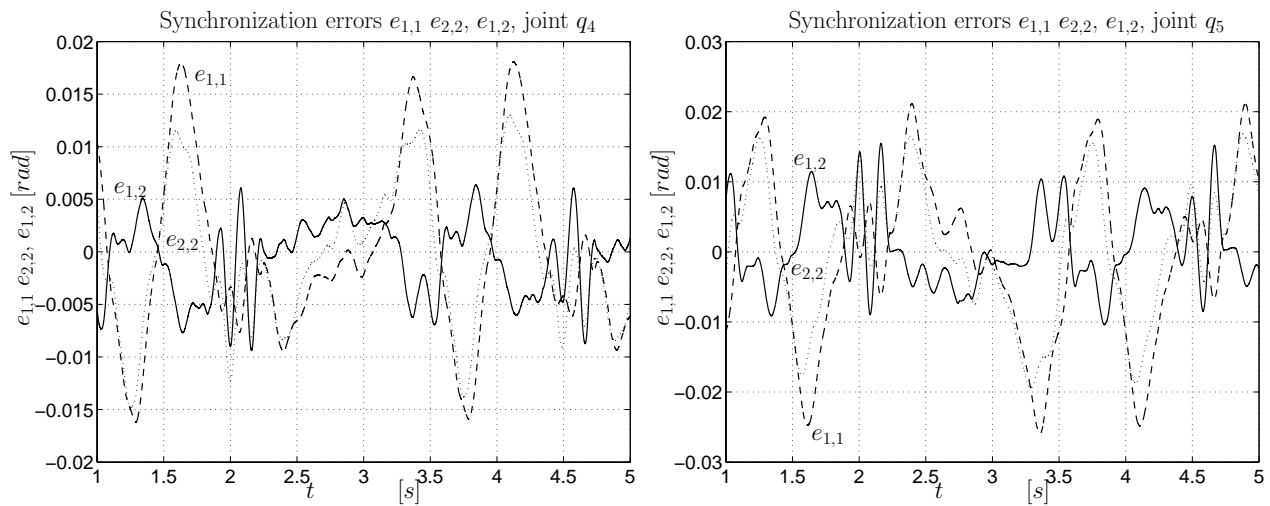

Figure 6.9: Synchronization errors $e_{1,1}$ (dashed), $e_{2,2}$ (dotted), and $e_{1,2}$ (solid), joints $q_{4}, q_{5}$. 


\subsection{Conclusions and discussion}

The external and mutual synchronization experiments that have been presented in this chapter lead to the following main conclusions.

- Synchronization, either external or mutual, of complex multi-robot systems can be achieved when only position measurements are available. Synchronization can be achieved by considering model based feedback controllers and observers.

- The proposed synchronization controller are model based. Therefore they are susceptible to errors in the modelling and identification of the robots, particularly for highly complex or coupled robots. However, the experiments show that the proposed synchronization controllers are robust against parametric uncertainties. Although the dynamic model (6.1) with the parameters listed in Table H.3 do not accurately model the robots, see validation Figures H.3 - H.6, the proposed controllers for external and mutual synchronization yield synchronization errors of the order of the resolution of the encoders.

- The controller and observer gains in the proposed synchronization controller can be physically interpreted. Thus they can be chosen intuitively during the tuning of the controllers. During the experiments it was observed that the gains $K_{p}$, $K_{p, i}, L_{p, 2}$ and $\mu_{i, 2}$ are all related to the feedback of the position measurements and the errors at position level. Therefore they have effects on the final bound of the synchronization errors. Since the positions are the only source of information in the system, these gains play the major role in the convergence and stability response of the synchronization closed loop system. On the other hand, the gains $K_{d}, K_{d, i}, L_{p, 1}$ and $\mu_{i, 1}$ are all related to feedback of variables at velocity level, thus they have effects on the overshoots and transient response. Since velocity is not measured, all those variables related to velocity level are result of estimation by observers. In case of noisy measurements in the system or bad estimated variables, large values of $K_{d}, K_{d, i}, L_{p, 1}$ and $\mu_{i, 1}$ lead to instability.

- During the experiments it was not attempted to obtain the best "performance" for the synchronization of the multi-robot system. Since the goal was to show that synchronization based only on position measurements is viable in complex systems, it is likely that for the specific multi-robot system some "performance" improvement can be accomplished. For instance the synchronization error $e_{1,2}$ for the joint $q_{5}$, see Figure 6.9(right), can be decreased by increasing the gain $K_{p, i}$. Nevertheless, the chosen set of gains illustrate the properties of the mutual synchronization controller, particularly how the errors $e_{1,1}, e_{2,2}$ are penalized to favor the error $e_{1,2}$. 


\section{Chapter 7}

\section{Extensions}

The ideas about synchronization of robot manipulators that have been presented in this thesis are general and can be extended to synchronization in other mechanical systems different from robot manipulators.

This chapter presents an extension of the external synchronization controller proposed in Chapter 3 to leader-follower mobile robots and an extension of the mutual synchronization controller proposed in Chapter 5 to attitude formations of multi-satellite systems. The extensions are presented in a general overview and without a complete stability analysis. The purpose of this chapter is to give some guidelines on how the proposed synchronization controllers can be modified to be applied to other systems different from robot manipulators.

Synchronization of the class leader-follower for mobile robots is presented in Section 7.1. Attitude formations of multi-satellite systems is addressed in Section 7.2. First a brief description of the dynamic model of the satellite is introduced, then the use of synchronization to achieve attitude formation of the members in a group of satellites systems is presented. The proposed extensions are validated by simulations.

The chapter closes with some remarks and discussions about other extensions for the synchronization problem addressed on this thesis.

\subsection{Leader-follower synchronization of mobile robots}

The control of mobile robots in Cartesian space is highly complicated because such systems are underactuated and subject to non-holonomic constraints. In the Cartesian space the mobile robot has 3 degrees of freedom while it has 2 external input controls. Nevertheless several tracking and stabilizing controllers have been proposed for those systems, e.g. (Canudas de Wit and Sordalen 1992), (Samson and Ait-Abderrahim 1991).

In this section we consider the particular controller presented in (Lefeber et al. 2001) to extend the ideas behind the external synchronization controller (master-slave scheme), Chapter 3, to a leader-follower configuration for mobile robots. Like in Chapter 3 the core of the synchronization controller is to use the measurements of the master (leader) system as desired trajectory for the slave system. We assume that one of the error 
coordinates is not measured, thus in the synchronization controller the unmeasured error coordinate is reconstructed by an observer.

The kinematic model of the mobile robots is presented in Section 7.1.1. Section 7.1.2 presents the proposed leader-follower synchronization controller. Simulation results presented in Section 7.1.3 validate the proposed synchronization controller.

\subsubsection{Kinematic model of the mobile robot}

The configuration of a mobile robot in the Cartesian space is given by

$$
q(t)=\left[\begin{array}{lll}
x(t) & y(t) & \theta(t)
\end{array}\right]
$$

where $q$ denotes the generalized Cartesian coordinates, $x(t)$ and $y(t)$ are the coordinates of the mass center of the mobile robot, and $\theta(t)$ denotes the heading angle. The kinematic model of the mobile robot in the Cartesian coordinates $q$ is given by

$$
\dot{x}=v \cos \theta, \quad \dot{y}=v \sin \theta, \quad \dot{\theta}=\omega
$$

where no-slip of the wheels is assumed, and the forward velocity $v$ and the angular velocity $\omega$ are considered as inputs.

The no-slip condition on the wheels imposes the non-holonomic constraint

$$
\dot{x} \sin \theta-\dot{y} \cos \theta=0
$$

As a result from Brockett's Theorem (Brockett 1983) the mobile robot modelled by (7.2) with the non-holonomic constraint (7.3) cannot be stabilized to a desired configuration via differentiable, or even continuous, pure-state feedback. Nevertheless there exist possible solutions to control such systems, for instance discontinuous feedback (Canudas de Wit and Sordalen 1992), time-varying continuous feedback (Samson and Ait-Abderrahim 1991), or the cascaded approach (Lefeber et al. 2001).

\subsubsection{Leader-follower synchronization controller}

Consider two mobile robots in a leader-follower configuration such that the subindices $l, f$ distinguish between the variables of the leader and follower system respectively. The kinematic model of the leader and follower mobile robot is given by (7.2), such that according to (Lefeber et al. 2001) the synchronization error coordinates between the systems can be expressed by

$$
\left[\begin{array}{l}
x_{e} \\
y_{e} \\
\theta_{e}
\end{array}\right]=\left[\begin{array}{ccc}
\cos \theta_{f} & \sin \theta_{f} & 0 \\
-\sin \theta_{f} & \cos \theta_{f} & 0 \\
0 & 0 & 1
\end{array}\right]\left[\begin{array}{c}
x_{l}-x_{f} \\
y_{l}-y_{f} \\
\theta_{l}-\theta_{f}
\end{array}\right]
$$

and the error dynamics is given by

$$
\begin{aligned}
& \dot{x}_{e}=\omega_{f} y_{e}-v_{f}+v_{l} \cos \theta_{e} \\
& \dot{y}_{e}=-\omega_{f} x_{e}+v_{l} \sin \theta_{e} \\
& \dot{\theta}_{e}=\omega_{l}-\omega_{f}
\end{aligned}
$$

Then the leader-follower problem can be stated as to design inputs $v_{f}$ and $\omega_{f}$ for the follower mobile robot such that the synchronization errors $x_{e}, y_{e}, \theta_{e}$ tend to zero. In 
certain pursuit navigation problems it might happen that one of the synchronization position errors $x_{e}, y_{e}$ cannot be measured, or at least not accurately. This of course complicates the leader-follower problem. Nevertheless the use of observers can solve the above problem by reconstructing the missing synchronization position error. In this section (as in (Lefeber et al. 2001)) we assume that we are unable to measure the forward synchronization error $x_{e}$, so only values of $y_{e}$ and $\theta_{e}$ are available. The case of unmeasured $y_{e}$ can be addressed analogously.

In (Lefeber et al. 2001) several tracking controllers and observers are proposed. We consider the particular combination of controller and full order observer given by

$$
\begin{aligned}
& \omega_{f}=\omega_{l}+c_{1} \theta_{e}, \quad c_{1}>0 \\
& v_{f}=v_{l}+c_{2} \widehat{x}_{e}-c_{3} \omega_{l} \widehat{y}_{e}, \quad c_{2}>0, c_{3}>-1
\end{aligned}
$$

where $\widehat{x}_{e}, \widehat{y}_{e}$ are generated by the observer

$$
\begin{aligned}
& \dot{\widehat{x}}_{e}=\omega_{l} \widehat{y}_{e}+v_{l}-v_{f}-l_{2} \omega_{l}\left(y_{e}-\widehat{y}_{e}\right), \quad l_{2}>-1 \\
& \dot{\hat{y}}_{e}=-\omega_{l} \widehat{x}_{e}+l_{1}\left(y_{e}-\widehat{y}_{e}\right) \quad l_{1}>0
\end{aligned}
$$

According to (Lefeber et al. 2001), if $\omega_{l}$ is persistently exciting (PE) then the closed loop system $(7.5,7.7,7.8)$ is globally uniformly exponentially stable (GUES).

\subsubsection{Simulation study}

The controller for the leader mobile robot $\omega_{l}, v_{l}$ is similar to the one for the follower mobile robot $(7.7),(7.8)$, and is given by

$$
\begin{aligned}
& \omega_{l}=\omega_{r}+c_{1} \theta_{e, l}, \quad c_{1}>0 \\
& v_{l}=v_{r}+c_{2} x_{e, l}-c_{3} \omega_{r} y_{e, l}, \quad c_{2}>0, c_{3}>-1
\end{aligned}
$$

where $\omega_{r}, v_{r}$ are the inputs on a model reference of the form given by (7.2) and $\left(x_{e, l}, y_{e, l}, \theta_{e, l}\right)$ are the tracking errors between the leader mobile robot and its reference, defined similarly to (7.4). The follower mobile robot is controlled by the proposed synchronization scheme (7.7), (7.8) together with the observer (7.9), (7.10). The control gains in the controller for the leader and follower mobile robot are listed in Table 7.1 .

\begin{tabular}{|c|c|c|c|c|c|}
\hline & $c_{1}$ & $c_{2}$ & $c_{3}$ & $l_{1}$ & $l_{2}$ \\
\hline leader & 6.0 & 1.5 & -0.4 & & \\
\hline follower & 20.0 & 25.0 & -0.5 & 10 & 50 \\
\hline
\end{tabular}

Table 7.1: Gains in the leader and follower controllers.

The leader mobile robot is commanded to move along a circle of radius 1 [m] with constant velocity, thus the inputs in the model reference are $\omega_{r}=1$ and $v_{r}=1$. The initial conditions in the leader and follower mobile robots are listed in Table 7.2. The initial conditions for the observer $(7.9,7.10)$ are set as $\widehat{x}_{e}(0)=0$ and $\widehat{y}_{e}(0)=0$.

Figures 7.1 - 7.3 show the Cartesian coordinates $x_{i}, y_{i}$ and $\theta_{i}$ for the leader $(i=l)$ and the follower $(i=f)$ mobile robots. The synchronization coordination errors $x_{e}, y_{e}$ and $\theta_{e}$ defined by (7.4) are shown as well. 


\begin{tabular}{|c|c|c|c|}
\hline$i=l, f$ & $x_{i}[\mathrm{~m}]$ & $y_{i}[\mathrm{~m}]$ & $\theta_{i}[\mathrm{rad}]$ \\
\hline leader & 0.5 & -0.5 & -1 \\
\hline follower & -0.2 & -0.75 & -2.0 \\
\hline
\end{tabular}

Table 7.2: Initial conditions of the mobile robots.
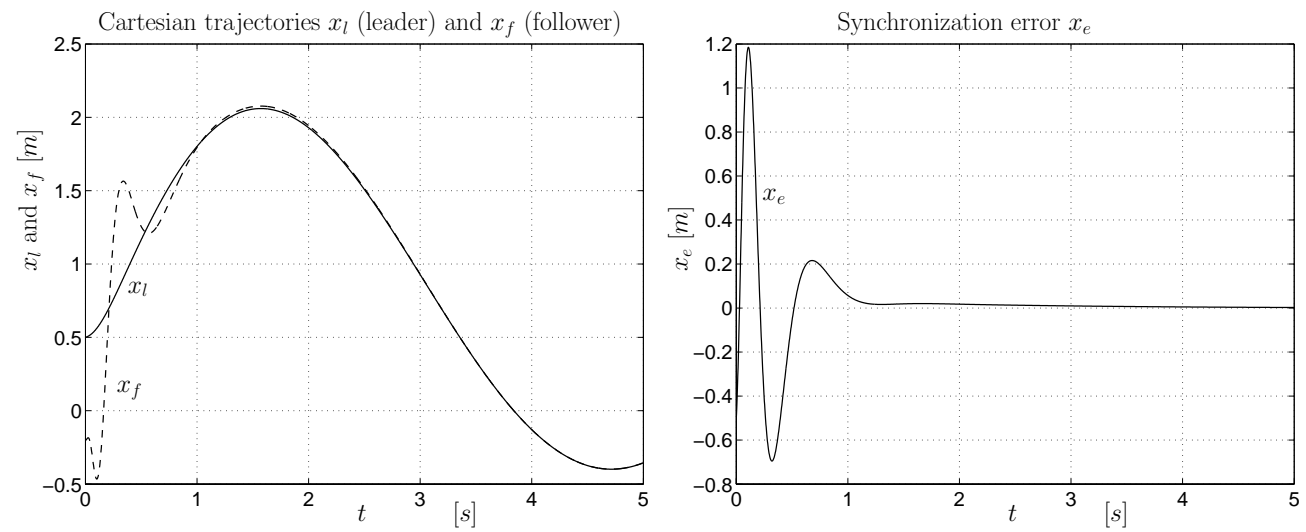

Figure 7.1: Cartesian coordinates $x_{l}$ (leader, solid), $x_{f}$ (follower, dashed), and synchronization error $x_{e}$
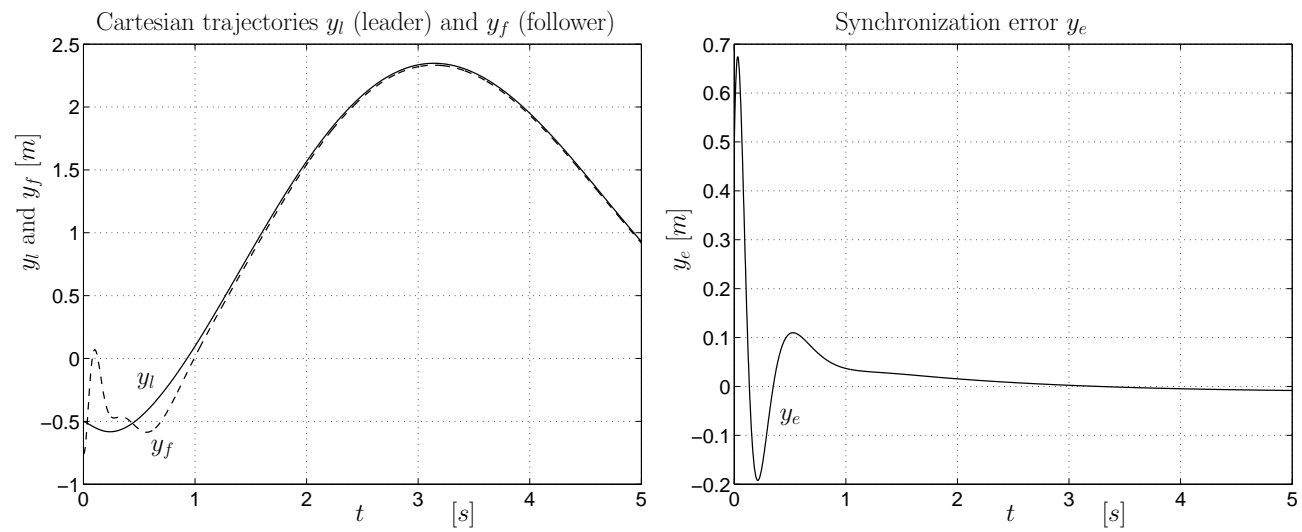

Figure 7.2: Cartesian coordinates $y_{l}$ (leader, solid), $y_{f}$ (follower, dashed), and synchronization error $y_{e}$ 

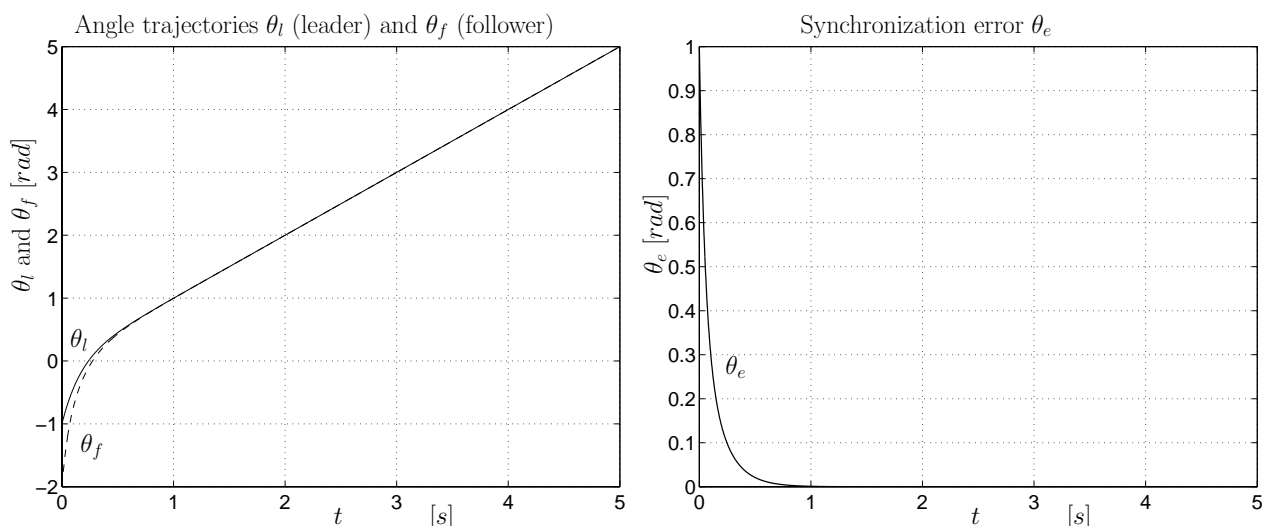

Figure 7.3: Heading angles $\theta_{l}$ (leader, solid), $\theta_{f}$ (follower, dashed), and synchronization error $\theta_{e}$

From Figures 7.1 - 7.3 it can be concluded that the Cartesian coordinates of the follower mobile robot match with the Cartesian coordinates for the leader mobile robot. Thus the leader-follower synchronization goal is achieved.

\subsection{Attitude formations of multi-satellite systems}

This section presents an extension of the mutual synchronization controller presented in Chapter 5 to the problem of attitude formations of multi-satellite systems. The proposed extension is based on the work presented in (Kang and Yeh 2002). In (Kang and Yeh 2002) a controller based on the so-called reference projection is proposed. The reference projection is nothing else but a definition of a synchronization goal equivalent to the nominal reference $q_{r i}$ defined by (5.7).

The proposed synchronization controller is decentralized and intended for multisatellites systems. The dynamics of a satellite systems is presented in Section 7.2.1, then the synchronization strategy and controller are proposed in Section 7.2.2. The proposed synchronization controller is validated by simulations in a two satellites system, Section 7.2.3.

\subsubsection{Dynamics of the satellite system}

To model the dynamics of a satellite two coordinate frames are introduced. The frame $E=\left\{E_{1}, E_{2}, E_{3}\right\}$ has its origin in the centre of mass of the satellite. The vectors $E_{i}$, $i=1,2,3$ point to fixed inertial directions. This frame system $E$ is almost identical to a fixed frame, except that its origin moves with the satellite. The origin of the rotating frame $e=\left\{e_{1}, e_{2}, e_{3}\right\}$ coincides with the origin of $E$, but the three axes of $e$ rotate with the satellite.

Let $S O(3)$ be the set of orthogonal $(3,3)$-matrices with determinant 1. A matrix $R \in S O(3)$ represents a rotation matrix that transforms $e$ into $E$, i.e.

$$
\left[\begin{array}{lll}
E_{1} & E_{2} & E_{3}
\end{array}\right]=\left[\begin{array}{lll}
e_{1} & e_{2} & e_{3}
\end{array}\right] R
$$

Therefore, a matrix in $S O(3)$ represents the orientation of a satellite. 
The dynamics of each satellite in the multi-satellite system is defined by the equations

$$
\begin{aligned}
\dot{R} & =S(\omega) R \\
J \dot{\omega} & =S(\omega) J \omega+u
\end{aligned}
$$

where $u=\left[\begin{array}{lll}u_{1} & u_{2} & u_{3}\end{array}\right]^{T}$ is the control torque and $J$ is the inertia matrix of the spacecraft. The vector $\omega=\left[\begin{array}{lll}\omega_{1} & \omega_{2} & \omega_{3}\end{array}\right] \in \mathbb{R}^{3}$ represents the angular velocity in the rotating frame, i.e.

$$
\omega=\omega_{1} e_{1}+\omega_{2} e_{2}+\omega_{3} e_{3}
$$

The matrix $S(\omega) \in \mathbb{R}^{3 \times 3}$ is defined by

$$
S(\omega)=\left[\begin{array}{ccc}
0 & w_{3} & -w_{2} \\
-w_{3} & 0 & w_{1} \\
w_{2} & -w_{1} & 0
\end{array}\right]
$$

Although the orthogonal matrix $R \in S O(3)$ is suitable to define the dynamics of the satellite (7.14), the use of quaternions is more convenient to define the desired trajectories and references for the satellites. Quaternions are coordinate systems used for the attitude of rigid bodies, More details about quaternions can be found in (Chovotov 1991). Different from $S O(3)$ quaternions involve the use of unit vectors in $\mathbb{R}^{4}$ to determine the attitude. A quaternion is a scalar plus a vector, totalling four elements. Three of the elements describe a vector, which defines an axis of rotation. The fourth element, a scalar, defines the magnitude of a rotation angle about the axis. Let a quaternion $q$ be defined as

$$
q=\left[\begin{array}{llll}
q_{0} & q_{1} & q_{2} & q_{3}
\end{array}\right]
$$

in which

$$
q_{0}=\cos \left(\frac{\mu}{2}\right), \quad q_{1}=m_{1} \sin \left(\frac{\mu}{2}\right), \quad q_{2}=m_{2} \sin \left(\frac{\mu}{2}\right), \quad q_{3}=m_{3} \sin \left(\frac{\mu}{2}\right)
$$

Where $m=\left[\begin{array}{lll}m_{1} & m_{2} & m_{3}\end{array}\right]^{T}$ is a unit vector representing the direction of the Euler vector, $\mu$ is the rotation angle about $m$. Such that $q_{o}$ represents the rotation angle in quaternions form, $q_{1}, q_{2}$ and $q_{3}$ represent the rotation angle $\mu$ around the vectors $m_{1}$, $m_{2}$ and $m_{3}$ which represent the axes $x, y$ and $z$ of the frame $E$ respectively. Since the rotation matrices $R \in S O(3)$ and the quaternions $q_{0}, q_{1}, q_{2}$ and $q_{3}$ represent a rotation about and axis, these representations are equivalent and there exist transformations from one to the other.

Suppose that $R=\left(r_{i j}\right)_{3 \times 3} \in S O(3)$ is the matrix of rotation in (7.13), then in terms of quaternions $q_{0}, q_{1}, q_{2}$ and $q_{3}$

$$
\begin{array}{r}
r_{11}=q_{1}^{2}-q_{2}^{2}-q_{3}^{2}+q_{0}^{2}, \quad r_{12}=2\left(q_{1} q_{2}+q_{3} q_{0}\right), \quad r_{13}=2\left(q_{1} q_{3}-q_{2} q_{0}\right) \\
r_{21}=2\left(q_{1} q_{2}-q_{3} q_{0}\right), \quad r_{22}=-q_{1}^{2}+q_{2}^{2}-q_{3}^{2}+q_{0}^{2}, \quad r_{23}=2\left(q_{1} q_{0}+q_{2} q_{3}\right) \\
r_{31}=2\left(q_{1} q_{3}+q_{2} q_{0}\right), \quad r_{32}=2\left(-q_{1} q_{0}+q_{2} q_{3}\right), \quad r_{33}=-q_{1}^{2}-q_{2}^{2}+q_{3}^{2}+q_{0}^{2}
\end{array}
$$

In terms of the components of a rotation matrix $R \in S O(3)$, the quaternions can be expressed as

$$
\begin{array}{ll}
q_{1}=\frac{1}{4 q_{0}}\left(r_{23}-r_{32}\right), & q_{2}=\frac{1}{4 q_{0}}\left(r_{31}-r_{13}\right) \\
q_{3}=\frac{1}{4 q_{0}}\left(r_{12}-r_{21}\right), & q_{0}= \pm \frac{1}{2}\left(1+r_{11}+r_{22}+r_{33}\right)^{\frac{1}{2}}
\end{array}
$$




\subsubsection{Synchronization strategy and controller}

In (Kang and Yeh 2002) a stabilizing feedback controller based on $H_{\infty}$-theory is proposed. A so-called reference projection is given to the feedback controller to achieve the synchronization behavior. The reference is defined based on the synchronization goal and the desired attitude trajectory. The synchronization controller proposed in this section is based on the feedback controller given in (Kang and Yeh 2002), the difference lies in the definition of the nominal reference (reference projection).

According to (Kang and Yeh 2002) a feedback controller in the rotating frame that stabilizes the attitude of the satellite is given by

$$
u\left(R, R_{e}\right)=-\frac{a}{2} \omega+\frac{b}{2} R \sum_{i=1}^{3} S\left(R_{e}^{T}(i)\right) R^{T}(i)
$$

where $a, b$ are positive scalar gains. $R^{T}(i)$ and $R_{e}^{T}(i)$ represent the $i$-th column vector of the transpose of the actual $R$ rotation matrix and the desired rotation matrix $R_{e}$, which is determined by the desired rotation angle $\mu_{d}$ about the desired axis. The desired rotation angle $\mu_{d}$ can be expressed in quaternions $q_{d}$ or rotation matrix $R_{e}$, since from the relations $(7.18-7.20)$ these representations are equivalent.

The synchronization mode between the systems is achieved by defining a nominal reference that is based on the synchronization goal. The nominal reference is given by the desired trajectory and terms that compensate for coupling errors between the systems to be synchronized. Consider for instance the nominal reference for $n$-robots $q_{r i}$ proposed in Chapter 5,

$$
q_{r i}=q_{d}-\sum_{j=1, j \neq i}^{p} K_{c p_{-} i, j}\left(q_{i}-q_{j}\right), \quad i=1, \ldots, n
$$

where $q_{d}$ is the common desired trajectory for the robots, and $K_{c p_{-} i, j}$ are coupling gains that multiply the partial synchronization errors $\left(q_{i}-q_{j}\right)$.

In (Kang and Yeh 2002) the so-called reference projection for a virtual desired trajectory (for two satellites) is proposed as

$$
R_{e i}=R\left(\frac{q_{d}+\rho\left(q_{j}-q_{i}\right)}{\left\|q_{d}+\rho\left(q_{j}-q_{i}\right)\right\|}\right), \quad i, j=1,2 \quad i \neq j
$$

where $q_{d}$ is the desired rotation, and $q_{1}, q_{2}$ are the actual rotation angle of the satellites, all represented in quaternions. The reference projection (7.23) determines a virtual desired attitude, which is a compromise between the attitude error $\left(q_{i}-\right.$ $q_{d}$ ) and the formation error $\left(q_{i}-q_{j}\right)$. However the virtual desired attitude is not necessarily close to the desired attitude given by the quaternions $q_{d}$. Moreover the reference projection can give rise to numerical problems in case the denominator $\left\|q_{d}+\rho\left(q_{j}-q_{i}\right)\right\|$ becomes zero.

Note the similarities between the references $q_{r i}$ and $R_{e i}$. Based on this similarities and to avoid the above mentioned problems in the definition of $R_{e i}$ (7.23), we propose a simpler nominal reference for the attitude formation problem as

$$
R_{e i}=R\left(q_{d}+\rho\left(q_{i}-q_{j}\right)\right), \quad i, j=1,2, \quad i \neq j
$$

It is worth to mention what the projection reference (7.23) and the nominal reference (7.24) represent and how they are obtained. The reference rotation matrix $R_{e i} \in$ 
$S O(3)$ represents a rotation of a satellite about one of its axes by an angle $\mu$, i.e. rotate the satellite and angle $\mu$ about one of the axes of frame $E$, such that the new orientation of the frame $e$ with respect to the frame $E$ is given by $R_{e i}$, see (7.13).

In (7.23) and (7.24) the rotation angle is expressed by means of quaternions (relation (7.18)). Such quaternions are used to define the rotation matrix $R(q)$ by considering the relationship (7.19). Also notice that the quaternions $q_{i}$ for the satellites are obtained from the actual rotation matrix $R$, given by (7.14), by considering the relationship (7.20). The use of quaternions in (7.23) and (7.24) simplifies the definition of the nominal reference. If the nominal reference is defined directly in the rotational matrices space $S O(3)$ then matrix multiplication and other mathematical complications related with matrix manipulation would arise.

As will be shown in the simulation study in the next subsection, the feedback controller (7.21) and the nominal reference (7.24) yield synchronous attitude formation of multisatellite systems.

\subsubsection{Simulation study}

The multi-satellite system in the simulation study is formed by two identical satellites with dynamics given by (7.14) and diagonal inertia matrix

$$
J=\left[\begin{array}{ccc}
1.0 & 0 & 0 \\
0 & 0.63 & 0 \\
0 & 0 & 0.85
\end{array}\right]
$$

The satellites rotate an angle $\mu_{i}(i=1,2)$ about the $z$-axis. The initial rotation angle of the satellites is $\mu_{1}=0.0[\mathrm{rad}]$ and $\mu_{2}=0.1[\mathrm{rad}]$ for satellites sat1 and sat2 respectively, such that the rotation matrices $R_{1}$ and $R_{2}$ are given by

$$
R_{1}=\left[\begin{array}{lll}
1 & 0 & 0 \\
0 & 1 & 0 \\
0 & 0 & 1
\end{array}\right], \quad R_{2}=\left[\begin{array}{ccc}
0.995 & 0.0998 & 0 \\
-0.0998 & 0.995 & 0 \\
0 & 0 & 1
\end{array}\right]
$$

The desired attitude of the satellites is

$$
R_{d}=\left[\begin{array}{ccc}
0.9801 & 0.1987 & 0 \\
-0.1987 & 0.9801 & 0 \\
0 & 0 & 1
\end{array}\right]
$$

which corresponds to a desired rotation angle $\mu_{d}=0.2[\mathrm{rad}]$ about the $z$-axis. By considering the relations (7.18) or equivalently (7.20) it follows that the desired rotation angle in quaternions is given by

$$
q_{0, d}=0.9950, \quad q_{1, d}=0, \quad q_{2, d}=0, \quad q_{3, d}=0.0998
$$

the quaternions $q_{1, d}$ and $q_{2, d}$ are zero because the rotation is about the $z$-axis, thus only the vector $m_{3}$ is considered in (7.18).

The synchronization or attitude formation error between the satellites $e_{f}$ is given by

$$
e_{f}=\mu_{1}-\mu_{2}
$$


The gains in the feedback controller for the satellites $i=1,2, u_{i}\left(R_{i}, R_{e i}\right)$, given by (7.21), are set as $a=10$ and $b=1$. The coupling gain $\rho$ in the nominal reference (7.24) is set as $\rho=1$ for synchronous control and $\rho=0$ for uncoupled satellites, i.e. neglecting the synchronous goal.

Figures 7.4 and 7.5 show the rotation angle $\mu_{1}$ and $\mu_{2}$ and the synchronization attitude formation error $e_{f}$ with and without synchronization, respectively.
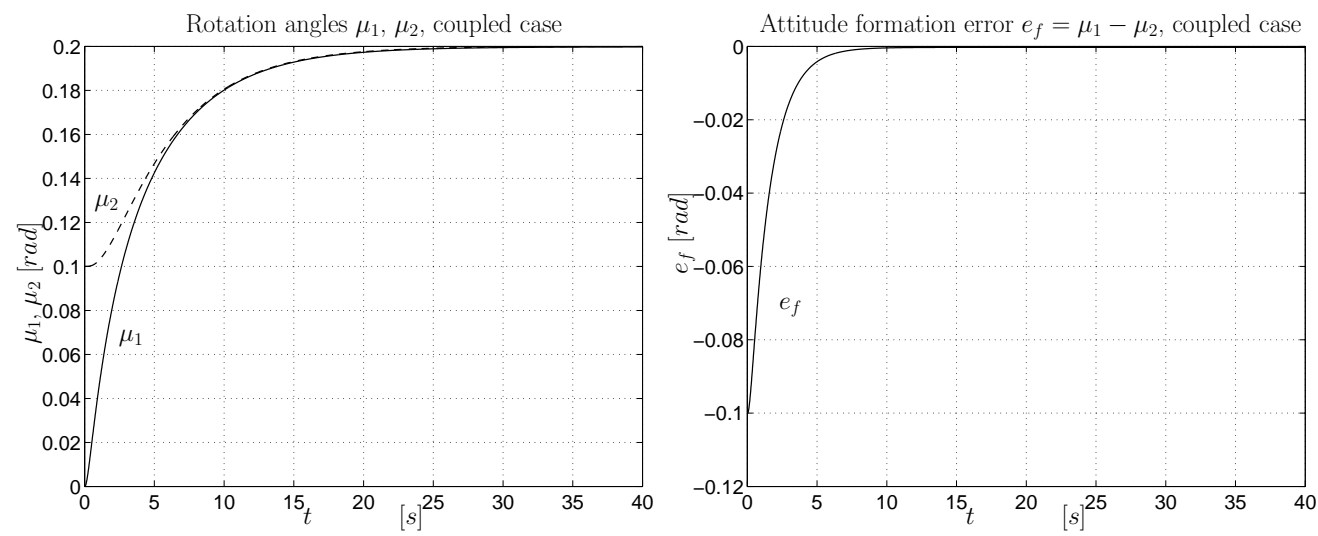

Figure 7.4: Angle rotations $\mu_{1}$ (solid), $\mu_{2}$ (dashed) and synchronization attitude error $e_{f}$, coupled case
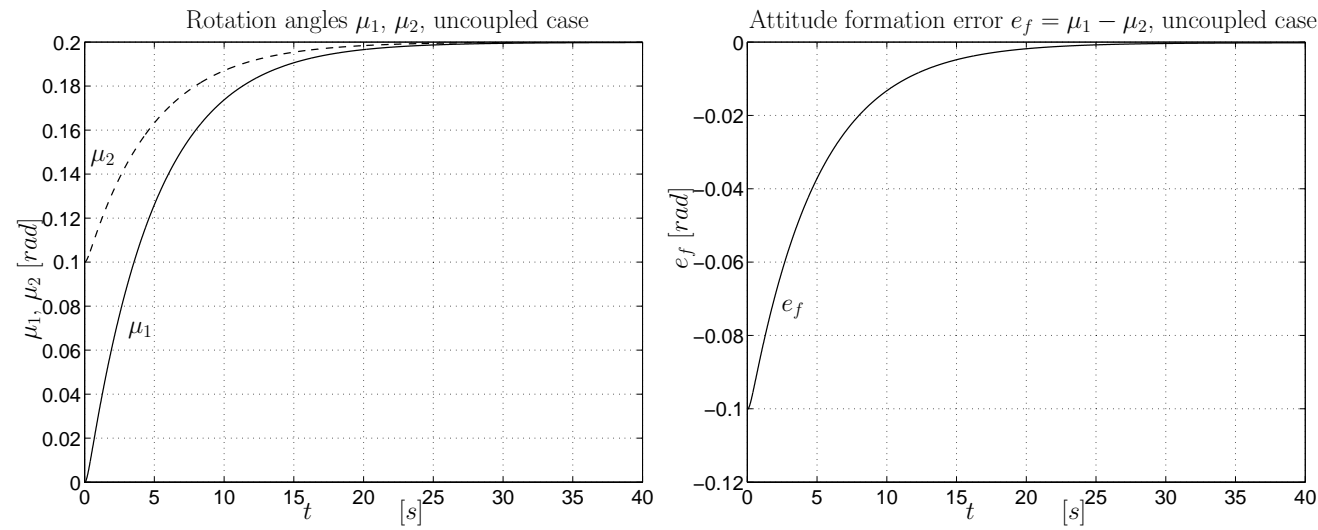

Figure 7.5: Angle rotations $\mu_{1}$ (solid), $\mu_{2}$ (dashed) and synchronization attitude error $e_{f}$, uncoupled case

From comparison between Figures 7.4 and 7.5 it is clear that the synchronization goal and the nominal reference (7.24) radically change the evolution of the attitude formation error $e_{f}=\mu_{1}-\mu_{2}$. The synchronization controller yields faster convergence between the satellites such that the attitude formation error $e_{f}$ between the satellites converges faster than the errors between the desired and actual rotation angles $\mu_{i}-\mu_{d}$, $i=1,2$. This makes the effects of the synchronization goal evident. 


\subsection{Discussion}

This chapter has presented extensions of the synchronization ideas proposed previously, particularly the external synchronization strategy, Chapter 3, to leader-follower mobile robots and the mutual synchronization strategy presented in Chapter 5 to multi-satellite systems. Clearly, the proposed ideas can be extended to other mechanical systems. For instance (Sun and Chiu 2002) proposed a synchronization controller for lifting platforms that are actuated by two electrohydraulic cylinders. The problem of coordination of multiple mobile robots has been addressed in (Hirata and Kosuge 2000) and (Siméon et al. 2002). Controlled synchronization of differential mobile robots has been studied in (Sun et al. 2002).

Although the simulation results presented in this chapter show that the definition of a synchronization goal together with a stabilizing or tracking controller can induce synchronization behavior in the system, this is not true in general. Since the synchronization goal is a function of the desired trajectory for a system and coupling errors between the systems, it implies a trade off between the systems to be synchronized. Thus if the synchronization behavior puts too much weight on the interaction between the systems and less on the desired trajectory, it may lead to instability or limit cycling. Particularly instability in the synchronization systems can be generated when there exists a contradiction between the coupled behaviors that are induced by the synchronization goal. Thus, in general the synchronization goal has to be formulated taking into account the nature of the systems and the proper couplings defined in it. And after the synchronization goal is defined a stability analysis of the synchronization closed loop system is required in each case.

The proposed leader-follower synchronization controller for mobile robots, Section 7.1 , is particularly interesting since it shows that synchronization can be achieved in underactuated (non-holonomic) systems. 


\section{Chapter 8}

\section{Conclusions and recommendations}

Many systems in nature present a tendency towards synchronization. But what is interesting from a control view point is to induce synchronization in a system or a group of individual systems. This controlled synchronization problem has been addressed in this thesis and this chapter presents general conclusions and recommendations for further extensions and research.

\subsection{Concluding remarks}

- The thesis proves analytically and experimentally that synchronization, either external or mutual, of multi-composed systems (particularly, fully actuated robot-manipulators) can be achieved when only position measurements are available. Synchronization can be achieved by considering model based feedback controllers and observers.

- Two classes of synchronization problems are studied in this thesis, external (Chapters 3 and 4) and mutual synchronization (Chapter 5). In external synchronization information is shared only from the leading system (master) to the follower systems (slaves), while in mutual synchronization there is a flow of information between all the involved systems. Mutual synchronization is based on the idea of defining synchronization goals based on coupling errors between all the systems.

The proposed mutual synchronization scheme controls the relationships between the position and velocities of the individual systems in the multi-composed system. In other words, the mutual synchronization goal is to regulate not only the convergence of the position and velocity of the individual system to the common desired trajectory, but also how the trajectories of each system converge with respect to each other. The mutual synchronization behavior has major effects during transients or sudden disturbances, so that the mutual synchronized multi-composed system presents better performance according to certain criteria such as convergence time, overshoots and coupled errors during transients. 
Two serious drawback of the mutual synchronization approach are the requirement of sharing information between the systems and the assumption that the dynamic model of the systems are known. In practical situations there is limited access to the systems and their variables. As proven in this thesis the problem of sharing information between the systems can be solved by using model based observers, although the requirement of the dynamic model of the systems remains as a problem.

- The assumption of availability of the dynamic model of all the systems has been relaxed in the proposed external synchronization controller, at least for master-slave systems, Chapters 3 and 4 . The proposed external synchronization controllers, Chapters 3 and 4, guarantee synchronization of the slave systems to the master system, when only the dynamic model of the slave systems and position measurements are available.

- The proposed synchronization controllers provide a systematic way of proving stability and boundedness of the synchronization closed loop system. The stability analysis shows that the synchronization result achieved is of a semi-global nature. In general a region of attraction can be established, and this region as well as the conditions on the controller and observer gains are quite conservative. Thus, there may exist more freedom on the initial errors and the controller gains than the ones predicted by the region of attraction and the proposed gain tuning procedures.

The outcome of the gain tuning procedures presented at the end of the Chapters 3 , 4, and 5 seems quite conservative, and only guarantees convergence and stability of the system. Therefore, once the boundaries of the controller and observer gains have been determined, these gains have to be chosen according to the desired transient and steady response of the synchronization system. As a guideline, first the gains in the observer can be chosen to obtain small estimation errors in the observers, then the gains in the feedback part of the synchronization controller can be tuned to achieved small synchronization errors. This tuning procedure resembles the "separation principle" that holds in linear systems, but unfortunately does not hold in general for nonlinear systems.

- Since the proposed synchronization controllers are model based, they present great dependence on the model of the systems to be synchronized. Therefore the synchronization controllers are susceptible to errors in the modelling and identification of the systems, particularly for highly complex or coupled systems. The proposed synchronization controllers proved to be robust against parametric uncertainties. Moreover the proposed controllers can be easily extended to include on-line adaptation of the parameters in the model of the systems. An adaptive version of the proposed controllers is obtained by considering linear parametric representations of the dynamic model of the systems.

- Although the thesis focuses on synchronization of multi-robot manipulators, the proposed synchronization ideas are quite general and applicable to different mechanical systems. Some extensions of the proposed synchronization controller to systems different from robot-manipulators, such as mobile robots and satellites, have been presented in Chapter 7. The extension to the leader-follower mobile robot problem presented in Section 7.1 is particularly interesting since it 
shows that synchronization can be induced in underactuated (non-holonomic) systems.

The proposed extensions presented in Chapter 7 show that the definition of a synchronization goal together with a stabilizing or tracking controller can induce synchronization in a multi-composed system, however this is not true in general. Since the synchronization goal includes the desired trajectory and coupled errors between the systems, it implies a trade off between the systems to be synchronized. Thus if the synchronization behavior puts too much weight on the interaction between the systems and less on the desired trajectory, it may lead to instability or limit cycling. Therefore a formal analysis of the stability and convergence of the synchronous behavior is required in each case.

\subsection{Recommendations}

The study and applications of synchronization phenomena have recently raised great interest in the control community. To understand why, consider the great variety of systems and applications in which synchronization improves the performance of the systems or allows a task to be executed. Therefore the study of synchronization phenomena and their applications are research problems which are not only challenging but motivating as well. In this section we list a few open problems in synchronization, particularly controlled synchronization, that directly arise from this thesis.

- A general framework that ensures synchronization of a large class of systems. Although the definition of a synchronization goal based on coupling errors combined with a stabilizing or tracking controller seems to be enough to induce synchronization, this is in general not true. Therefore a general framework and conditions in which such combination, synchronization goal plus controller, yields synchronization are required. Such general framework should provide a synchronization control strategy for a large variety of systems more than for particular cases as the actual state of art is.

- Information shared between the systems. The synchronization goal and coupling errors are based on sharing information between the systems to be synchronized. But the information that is shared may be redundant according to the synchronization goal and therefore the question that arises is: what is the minimum amount of information necessary to achieve synchronization?

- Definition of the synchronization goal. The first step in a controlled synchronization problem is to define the synchronization goal. This goal not only determines the desired synchronous behavior of the system, but most important from a control view point, it gives an idea of the information that has to be shared between the systems and how this information is given to the controller. For instance one can define a synchronization goal such that full synchronization or partial synchronization is achieved. Partial synchronization is particularly interesting since it allows clustering of a multi-composed system. Thus certain subsets of the multi-composed system may be working in a synchronous mode, whereas other subsets present a different synchronous mode.

Another example is formations of mobile systems. One could define two or more mobile systems being synchronized when they keep a fixed distance between 
them. Also keeping the velocity and heading angles equal for all the systems can be considered as synchronization.

- Definition of the reference trajectories that force the synchronization via the feedback controller. The synchronous behavior in a controlled synchronization system is determined by the synchronization functional and synchronization goal, which can be used to define the nominal reference or the so-called projection reference that is given to the controller. As a rule of thumb the nominal reference is a function of the desired trajectory and some terms that compensate for coupling errors between the individual systems. The coupling errors are in charge of the synchronous behavior, whereas the desired trajectory determines the task to be executed by the synchronized systems. In this thesis the nominal reference has been defined as a sum of the coupling errors, but different definitions for the nominal reference can be considered. For instance the integral of the coupling errors, or a polynomial function, etc., would result in a different synchronization functional.

- Synchronization of underactuated systems . The robot manipulators considered in the thesis are fully actuated. But an obvious question is under what conditions synchronization can be induced in underactuated systems. The extension proposed for leader-follower mobile robots, Section 7.1, shows that such underactuated (non-holonomic) system can be synchronized. However many questions are open such as what kind of underactuated systems are suitable for synchronization, either external or mutual; which kind of constraints should be satisfied by the non-actuated part of the system, etc..

In principle the constrains that are imposed by the underactuated part of the system should lie in the manifold define by the synchronization goal. If that is not the case it is impossible for the dynamics of the system to achieve the synchronization goal keeping the dynamics in the constraints. 


\section{Appendix A}

\section{Proof of Lemma 3.9}

In this appendix we develop the synchronization closed loop dynamics given by $(3.22$, 3.23 , and 3.24). First, we obtain the error dynamics in terms of the synchronization errors $(e, \dot{e})$, the estimation synchronization errors $(\widetilde{e}, \widetilde{e})$, and the estimation position and velocity errors $\left(\widetilde{q}_{s}, \widetilde{\dot{q}}_{s}\right)$, and second we consider the coordinate transformation defined by (3.16), (3.17).

\section{Synchronization error dynamics}

Substitution of $\tau_{s}(3.9)$ in (3.1), by adding and subtracting $K_{d} \dot{e}+M_{s}\left(q_{s}\right) \ddot{q}_{m}+$ $C_{s}\left(q_{s}, \dot{q}_{s}\right) \dot{q}_{m}$, and considering the synchronization errors defined by (3.7), we obtain that

$$
\begin{aligned}
M_{s}\left(q_{s}\right) \ddot{e}+ & C_{s}\left(q_{s}, \dot{q}_{s}\right) \dot{e}+K_{d} \dot{e}+K_{p} e=M_{s}\left(q_{s}\right)\left(\widehat{\ddot{q}}_{m}-\ddot{q}_{m}\right) \\
& +C_{s}\left(q_{s}, \widehat{\dot{q}}_{s}\right) \widehat{\hat{q}}_{m}-C_{s}\left(q_{s}, \dot{q}_{s}\right) \dot{q}_{m}-K_{d}(\hat{\dot{e}}-\dot{e})
\end{aligned}
$$

From $(3.7),(3.11),(3.13)$, and (3.14), the following equalities can be established

$$
\begin{aligned}
\widehat{q}_{m}-q_{m} & =\widetilde{e}-\widetilde{q}_{s} \\
\widehat{\dot{q}}_{m}-\dot{q}_{m} & =\widetilde{\dot{e}}-\widetilde{\dot{q}}_{s} \\
\widehat{\ddot{q}}_{m}-\ddot{q}_{m} & =\frac{d}{d t}\left(\widetilde{\dot{e}}-\widetilde{\dot{q}}_{s}\right)
\end{aligned}
$$

Considering (3.11), (3.13), (A.2) and Property (2.27), it follows that

$$
\begin{gathered}
C_{s}\left(q_{s}, \widehat{\dot{q}}_{s}\right) \widehat{\dot{q}}_{m}-C_{s}\left(q_{s}, \dot{q}_{s}\right) \dot{q}_{m}=C_{s}\left(q_{s}, \dot{q}_{s}\right) \widetilde{\dot{e}}-2 C_{s}\left(q_{s}, \dot{q}_{s}\right) \widetilde{\dot{q}}_{s} \\
+C_{s}\left(q_{s}, \widetilde{\dot{q}}_{s}\right) \widetilde{\dot{q}}_{s}+C_{s}\left(q_{s}, \widetilde{\dot{q}}_{s}\right) \dot{e}-C_{s}\left(q_{s}, \widetilde{\dot{q}}_{s}\right) \widetilde{\dot{e}}^{2}
\end{gathered}
$$

Substitution of (A.3) in (A.1), and considering (3.11), (A.2), yields

$$
\begin{aligned}
M_{s}\left(q_{s}\right) \ddot{e}+ & C_{s}\left(q_{s}, \dot{q}_{s}\right) \dot{e}+K_{d} \dot{e}+K_{p} e=M_{s}\left(q_{s}\right) \frac{d}{d t}\left(\widetilde{\dot{e}}-\widetilde{\dot{q}}_{s}\right) \\
& +K_{d} \widetilde{\dot{e}}-2 C_{s}\left(q_{s}, \dot{q}_{s}\right) \widetilde{\dot{q}}_{s}+C_{s}\left(q_{s}, \widetilde{\dot{q}}_{s}\right) \widetilde{\dot{q}}_{s}+C_{s}\left(q_{s}, \widetilde{\dot{q}}_{s}\right) \dot{e} \\
& -C_{s}\left(q_{s}, \widetilde{\dot{q}}_{s}\right) \widetilde{\dot{e}}+C_{s}\left(q_{s}, \dot{q}_{s}\right) \widetilde{\dot{e}}
\end{aligned}
$$




\section{Estimation synchronization error dynamics}

Define states $x_{1}, x_{2} \in \mathbb{R}^{n}$ as $x_{1}:=e, x_{2}:=\dot{e}$, and obtain a state space representation for (A.4). In the states $x_{1}, x_{2}$ the estimation synchronization errors (3.11) are given by

$$
\widetilde{e}=x_{1}-\widehat{e}, \quad \widetilde{\dot{e}}=x_{2}-\widehat{\dot{e}}
$$

Therefore, from the state space representation of (A.4) and the observer defined by (3.10), the estimation synchronization error dynamics are given by

$$
\begin{aligned}
\frac{d}{d t} \widetilde{e}=\widetilde{e} & -\Lambda_{1} \widetilde{e} \\
\frac{d}{d t} \widetilde{\dot{e}}= & M_{s}\left(q_{s}\right)^{-1}\left\{-C_{s}\left(q_{s}, \dot{q}_{s}\right) x_{2}-K_{d} x_{2}-K_{p} x_{1}+M_{s}\left(q_{s}\right) \frac{d}{d t}\left(\widetilde{\dot{e}}-\widetilde{\dot{q}}_{s}\right)\right. \\
& +C_{s}\left(q_{s}, \dot{q}_{s}\right) \widetilde{\dot{e}}+C_{s}\left(q_{s}, \hat{\dot{q}}_{s}\right) \hat{\dot{e}}+\left(C_{s}\left(q_{s}, \tilde{\dot{q}}_{s}\right)-2 C_{s}\left(q_{s}, \dot{q}_{s}\right)\right) \widetilde{\dot{q}}_{s} \\
& \left.+C_{s}\left(q_{s}, \widetilde{\dot{q}}_{s}\right)\left(x_{2}-\widetilde{\dot{e}}\right)+K_{d} \widetilde{\dot{e}}+K_{d} \widehat{\dot{e}}+K_{p} \widehat{e}\right\}-\Lambda_{2} \widetilde{e}
\end{aligned}
$$

Considering (3.13), (A.2), (A.5) and after a straightforward computation, these equations reduce to

$$
\begin{aligned}
\frac{d}{d t} \widetilde{e} & =\widetilde{e}-\Lambda_{1} \widetilde{e} \\
\frac{d}{d t} \widetilde{\dot{q}}_{s} & =M_{s}\left(q_{s}\right)^{-1}\left\{-K_{p} \widetilde{e}-2 C_{s}\left(q_{s}, \dot{q}_{s}\right) \widetilde{\dot{q}}_{s}+C_{s}\left(q_{s}, \widetilde{\dot{q}}_{s}\right) \widetilde{\dot{q}}_{s}\right\}-\Lambda_{2} \widetilde{e}
\end{aligned}
$$

\section{Estimation joint position and velocity error dynamics}

From the definition of the synchronization errors (3.7), it follows that

$$
\ddot{q}_{s}=\ddot{e}+\ddot{q}_{m}
$$

Define states $z_{1}, z_{2} \in \mathbb{R}^{n}$ as $z_{1}:=q_{s}, z_{2}:=\dot{q}_{s}$, and obtain a state space representation for (A.4). In the states $z_{1}, z_{2}$ the estimation position and velocity errors (3.13) are given by

$$
\widetilde{q}_{s}=z_{1}-\widehat{q}_{s}, \quad \widetilde{\dot{q}}_{s}=z_{2}-\widehat{\dot{q}}_{s}
$$

So, from the state space representation for (A.4), with states $z_{1}, z_{2}$, and observer (3.12), the estimation position and velocity error dynamics are given by

$$
\begin{aligned}
\frac{d}{d t} \widetilde{q}_{s}= & \widetilde{\dot{q}}_{s}-L_{p 1} \widetilde{q}_{s} \\
\frac{d}{d t} \widetilde{\dot{q}}_{s}= & M_{s}\left(q_{s}\right)^{-1}\left\{-\left(C_{s}\left(q_{s}, z_{2}\right)+K_{d}\right) \dot{e}-K_{p} e+M_{s}\left(q_{s}\right) \frac{d}{d t}\left(\widetilde{\dot{e}}^{-} \widetilde{\dot{q}}_{s}\right)\right. \\
& +C_{s}\left(q_{s}, z_{2}\right) \widetilde{\dot{e}}+\left(C_{s}\left(q_{s}, \widetilde{\dot{q}}_{s}\right)-2 C_{s}\left(q_{s}, z_{2}\right)\right) \widetilde{\dot{q}}_{s}+C_{s}\left(q_{s}, \widetilde{\dot{q}}_{s}\right) \dot{e} \\
& \left.-C_{s}\left(q_{s}, \widetilde{\dot{q}}_{s}\right) \widetilde{\dot{e}}+C_{s}\left(q_{s}, \widehat{\dot{q}}_{s}\right) \widehat{\dot{e}}+K_{d} \widetilde{\dot{e}}+K_{d} \widehat{\dot{e}}+K_{p} e\right\}-L_{p 2} \widetilde{q}_{s}+\ddot{q}_{m}
\end{aligned}
$$

Considering (3.13), (A.2), and (A.9), these equations reduce to

$$
\begin{aligned}
\frac{d}{d t} \widetilde{q}_{s}= & \widetilde{\dot{q}}_{s}-L_{p 1} \widetilde{q}_{s} \\
\frac{d}{d t} \widetilde{\dot{q}}_{s}= & \frac{d}{d t}\left(\widetilde{\dot{e}}-\widetilde{\dot{q}}_{s}\right)+M_{s}\left(q_{s}\right)^{-1}\left\{-2 C_{s}\left(q_{s}, z_{2}\right) \widetilde{\dot{q}}_{s}+C_{s}\left(q_{s}, \widetilde{\dot{q}}_{s}\right) \widetilde{\dot{q}}_{s}\right\} \\
& -L_{p 2} \widetilde{q}_{s}+\ddot{q}_{m}
\end{aligned}
$$


Finally, from (A.7) and (A.11), it follows that

$$
\begin{aligned}
\frac{d}{d t} \widetilde{q}_{s}= & \widetilde{\dot{q}}_{s}-L_{p 1} \widetilde{q}_{s} \\
\frac{d}{d t} \widetilde{\dot{e}}= & M_{s}\left(q_{s}\right)^{-1}\left\{-2 K_{p} \widetilde{e}-2 C_{s}\left(q_{s}, \dot{q}_{s}\right) \widetilde{\dot{q}}_{s}+C_{s}\left(q_{s}, \widetilde{\dot{q}}_{s}\right) \widetilde{\dot{q}}_{s}\right\} \\
& -2 \Lambda_{2} \widetilde{e}+L_{p 2} \widetilde{q}_{s}-\ddot{q}_{m}
\end{aligned}
$$

where the fact that $z_{2}=\dot{q}_{s}$ has been used.

\section{Coordinate transformations}

Consider the coordinate transformation defined by (3.16), subtraction of (A.7, A.12) from (A.6, A.13) gives rise to the dynamics for $\widetilde{q}_{m}, \dot{\widetilde{q}}_{m}$

$$
\begin{aligned}
\frac{d}{d t} \widetilde{q}_{m} & =\widetilde{\dot{e}}-\widetilde{\dot{q}}_{s}-L_{p 1} \widetilde{q}_{m} \\
\frac{d}{d t}\left(\widetilde{\dot{e}}-\widetilde{\dot{q}}_{s}\right) & =-M_{s}\left(q_{s}\right)^{-1} K_{p}\left(\widetilde{q}_{m}+\widetilde{q}_{s}\right)-L_{p 2} \widetilde{q}_{m}-\ddot{q}_{m}
\end{aligned}
$$

where Assumption 3.5 has been used.

From (A.7), (A.12), it follows that

$$
\begin{aligned}
\frac{d}{d t} \widetilde{q}_{s}= & \widetilde{\dot{q}}_{s}-L_{p 1} \widetilde{q}_{s} \\
\frac{d}{d t} \widetilde{\dot{q}}_{s}= & M_{s}\left(q_{s}\right)^{-1}\left\{-K_{p}\left(\widetilde{q}_{m}+\widetilde{q}_{s}\right)-2 C_{s}\left(q_{s}, \dot{q}_{s}\right) \widetilde{\dot{q}}_{s}+C_{s}\left(q_{s}, \widetilde{\dot{q}}_{s}\right) \widetilde{\dot{q}}_{s}\right\} \\
& -L_{p 2}\left(\widetilde{q}_{m}+\widetilde{q}_{s}\right)
\end{aligned}
$$

From the last four equations we obtain the error dynamics (3.23) and (3.24). By adding and subtracting $K_{p} \widetilde{q}_{m}+C_{s}\left(q_{s}, \dot{q}_{s}\right) L_{p 1} \widetilde{q}_{m}+K_{d} L_{p 1} \widetilde{q}_{m}+M\left(q_{s}\right)\left(L_{p 1} \dot{\widetilde{q}}_{m}-L_{p 1} L_{p 1} \widetilde{q}_{m}\right)$ from (A.4), and considering the coordinate transformation defined by (3.16) and (3.17), this yields (3.22). 


\section{Appendix B}

\section{Proof of Theorem 3.12}

In this appendix we present the details behind the proof of the Theorem 3.12.

\section{Time derivative of the Lyapunov function $V(y)$}

Consider the Lyapunov function $V(y)$ defined by (3.29), along the error dynamics (3.22 - 3.24), and by using Assumption 3.6, the time derivative of (3.29) becomes

$$
\dot{V}(y)=-y^{T} Q(y) y+\beta\left(y, \dot{q}_{s}, \ddot{q}_{m}\right)
$$

where

$$
\begin{aligned}
& \beta\left(y, \dot{q}_{s}, \ddot{q}_{m}\right)=\varepsilon_{o}\left(\dot{\bar{q}}^{T}+\lambda_{o} \bar{q}^{T}\right) C_{s}\left(q_{s}, \dot{\tilde{q}}_{s}+L_{p 1} \widetilde{q}_{s}\right)\left(\dot{\bar{q}}-L_{p 1} \widetilde{q}_{m}\right) \\
& \quad+\varepsilon_{o} \dot{\bar{q}}^{T} C_{s}\left(q_{s}, \dot{q}_{s}\right) L_{p 1} \widetilde{q}_{m}-\varepsilon_{o} \lambda_{o} \bar{q}^{T} C_{s}\left(q_{s}, \dot{q}_{s}\right)\left(\dot{\bar{q}}-L_{p 1} \widetilde{q}_{m}\right)+\left(\dot{\widetilde{q}}_{s}^{T}+\gamma \widetilde{q}_{s}^{T}\right) \\
& \quad \times M_{s}\left(q_{s}\right)^{-1}\left(C_{s}\left(q_{s}, \dot{\widetilde{q}}_{s}+L_{p 1} \widetilde{q}_{s}\right)-2 C_{s}\left(q_{s}, \dot{q}_{s}\right)\right)\left(\dot{\widetilde{q}}_{s}+L_{p 1} \widetilde{q}_{s}\right) \\
& \quad+\varepsilon_{o} \lambda_{o} \dot{\bar{q}}^{T} \dot{M}_{s}\left(q_{s}\right) \bar{q}+\dot{\mu} \dot{\widetilde{q}}_{m}^{T} \widetilde{q}_{m}+\dot{\gamma} \dot{\widetilde{q}}_{s}^{T} \widetilde{q}_{s}-\left(\dot{\widetilde{q}}_{m}^{T}+\mu \widetilde{q}_{m}^{T}\right) \ddot{q}_{m}
\end{aligned}
$$

and $Q(y)=Q(y)^{T}$ is given by

$$
Q(y)=\left[\begin{array}{lll}
Q_{11} & Q_{12} & Q_{13} \\
Q_{12}^{T} & Q_{22} & Q_{23} \\
Q_{13}^{T} & Q_{23}^{T} & Q_{33}
\end{array}\right]
$$

with the block matrices

$$
\begin{gathered}
Q_{11}=\varepsilon_{o}\left[\begin{array}{cc}
K_{d}-\lambda_{o} M_{s}\left(q_{s}\right) & 0 \\
0 & \lambda_{o} K_{p}
\end{array}\right] \\
Q_{12}=\frac{\varepsilon_{o}}{2}\left[\begin{array}{cc}
-M_{s}\left(q_{s}\right) L_{p 1} & K_{p}-K_{d} L_{p 1} \\
-\lambda_{o} M_{s}\left(q_{s}\right) L_{p 1} & \lambda_{o}\left(K_{p}-K_{d} L_{p 1}\right)
\end{array}\right] \\
Q_{13}=\frac{\varepsilon_{o}}{2}\left[\begin{array}{cc}
-K_{d} & -K_{d} L_{p 1} \\
-\lambda_{o} K_{d} & -\lambda_{o} K_{d} L_{p 1}
\end{array}\right]
\end{gathered}
$$




$$
\begin{gathered}
Q_{22}=\left[\begin{array}{cc}
L_{p 1}-\mu I & \frac{1}{2}\left(M_{s}\left(q_{s}\right)^{-1} K_{p}+\mu L_{p 1}\right) \\
\frac{1}{2}\left(M_{s}\left(q_{s}\right)^{-1} K_{p}+\mu L_{p 1}\right)^{T} & \mu\left(M_{s}\left(q_{s}\right)^{-1} K_{p}+L_{p 2}\right)
\end{array}\right] \\
Q_{23}=\left[\begin{array}{cc}
0 & \frac{1}{2} M_{s}\left(q_{s}\right)^{-1} K_{p} \\
\frac{1}{2}\left(M_{s}\left(q_{s}\right)^{-1} K_{p}+L_{p 2}\right) & \frac{1}{2}\left((\mu+\gamma) M_{s}\left(q_{s}\right)^{-1} K_{p}+\gamma L_{p 2}\right)
\end{array}\right] \\
Q_{33}=\left[\begin{array}{cc}
L_{p 1}-\gamma I & \frac{1}{2}\left(M_{s}\left(q_{s}\right)^{-1} K_{p}+\gamma L_{p 1}\right) \\
\frac{1}{2}\left(M_{s}\left(q_{s}\right)^{-1} K_{p}+\gamma L_{p 1}\right)^{T} & \gamma\left(M_{s}\left(q_{s}\right)^{-1} K_{p}+L_{p 2}\right)
\end{array}\right]
\end{gathered}
$$

To conclude stability of the variable $y$ defined by (3.19), we require positive definiteness of $Q(y)$ and boundedness of the term $\beta\left(y, \dot{q}_{s}, \ddot{q}_{m}\right)$ along the synchronization closed loop error dynamics (3.22 - 3.24).

\section{Boundedness of $\beta\left(y, \dot{q}_{s}, \ddot{q}_{m}\right)$}

First, from the definition of $\mu\left(\widetilde{q}_{m}\right), \gamma\left(\widetilde{q}_{s}\right)(3.30)$, it follows that

$$
\begin{gathered}
\dot{\mu} \dot{\widetilde{q}}_{m}^{T} \widetilde{q}_{m}=-\mu\left(\frac{\widetilde{q}_{m}^{T} \dot{\widetilde{q}}_{m}}{1+\left\|\widetilde{q}_{m}\right\|}\right) \dot{\tilde{q}}_{m}^{T} \widetilde{q}_{m} \leq \mu\left\|\dot{\tilde{q}}_{m}\right\|^{2} \\
\dot{\gamma} \dot{\tilde{q}}_{s}^{T} \widetilde{q}_{s}=-\gamma\left(\frac{\widetilde{q}_{s}^{T} \dot{\widetilde{q}}_{s}}{1+\left\|\widetilde{q}_{s}\right\|}\right) \dot{\tilde{q}}_{s}^{T} \widetilde{q}_{s} \leq \gamma\left\|\dot{\tilde{q}}_{s}\right\|^{2}
\end{gathered}
$$

Then by boundedness of $\mu\left(\widetilde{q}_{m}\right), \gamma\left(\widetilde{q}_{s}\right)$ (3.31) it is obtained that

$$
\dot{\mu} \dot{\widetilde{q}}_{m}^{T} \widetilde{q}_{m} \leq \mu_{o}\left\|\dot{\widetilde{q}}_{m}\right\|^{2}, \quad \text { and } \quad \dot{\gamma} \dot{\widetilde{q}}_{s}^{T} \widetilde{q}_{s} \leq \gamma_{o}\left\|\dot{\widetilde{q}}_{s}\right\|^{2}
$$

On the other hand, the definition of the synchronization errors (3.7) implies that

$$
\dot{q}_{s}=\dot{e}+\dot{q}_{m}
$$

Then, from the definition of $\dot{\bar{q}}$, (3.17), we obtain a relation between $\dot{q}_{s}$ and $\dot{\bar{q}}$, which is given by

$$
\dot{q}_{s}=\dot{\bar{q}}+\dot{\widetilde{q}}_{m}+\dot{q}_{m}
$$

Finally, the definition of the inertia matrix $M_{s}\left(q_{s}\right)$ implies that

$$
\dot{M}_{s}\left(q_{s}\right)=\frac{d}{d t} M_{s}\left(q_{s}\right)=\frac{\partial M_{s}\left(q_{s}\right)}{\partial q_{s}} \dot{q}_{s}
$$

hence, by property (2.28) and since $q_{s}$ only appears like argument of trigonometric functions in $M_{s}\left(q_{s}\right)$, i.e. sine and cosine functions, we conclude that

$$
M_{s, p m}\left\|\dot{q}_{s}\right\| \leq\left\|\dot{M}_{s}\left(q_{s}\right)\right\| \leq M_{s, p M}\left\|\dot{q}_{s}\right\|
$$

where

$$
M_{s, p m} \leq\left\|\frac{\partial M_{s}\left(q_{s}\right)}{\partial q_{s}}\right\| \leq M_{s, p M}
$$


Then, from (B.6 - B.8), properties (2.28), (2.29), and taking into account Assumption 3.11 , it follows that $\beta\left(y, \dot{q}_{s}, \ddot{q}_{m}\right)$ has an upperbound given by

$$
\begin{aligned}
& \beta\left(y, \dot{q}_{s}, \ddot{q}_{m}\right) \leq-\varepsilon_{o} \lambda_{o} C_{s, M}\|\bar{q}\|\left(\|\dot{\bar{q}}\|-L_{p 1, M}\left\|\widetilde{q}_{m}\right\|\right)\left(\|\dot{\bar{q}}\|+\left\|\widetilde{q}_{m}\right\|+V_{M}\right) \\
& +\varepsilon_{o} C_{s, M} L_{p 1, M}\|\dot{\bar{q}}\|\left\|\widetilde{q}_{m}\right\|\left(\|\dot{\bar{q}}\|+\left\|\widetilde{q}_{m}\right\|+V_{M}\right)-A_{M}\left(\left\|\dot{\widetilde{q}}_{m}\right\|+\mu_{o}\left\|\widetilde{q}_{m}\right\|\right) \\
& +\varepsilon_{o} C_{s, M}\left(\|\dot{\bar{q}}\|+\lambda_{o}\|\bar{q}\|\right) \\
& \times\left(\left\|\dot{\widetilde{q}}_{s}\right\|\left(\|\dot{\bar{q}}\|-L_{p 1, m}\left\|\widetilde{q}_{m}\right\|\right)+\left\|\widetilde{q}_{s}\right\|\left(L_{p 1, M}\|\dot{\bar{q}}\|-L_{p 1, m}^{2}\left\|\widetilde{q}_{m}\right\|\right)\right) \\
& -2 M_{s, m}^{-1} C_{s, M}\left(\left\|\dot{\widetilde{q}}_{s}\right\|+\gamma_{o}\left\|\widetilde{q}_{s}\right\|\right)\left(\left\|\dot{\widetilde{q}}_{s}\right\|+L_{p 1, M}\left\|\widetilde{q}_{s}\right\|\right) \\
& \times\left(\|\dot{\bar{q}}\|+\left\|\widetilde{q}_{m}\right\|+V_{M}\right)+\varepsilon_{o} \lambda_{o} M_{s, p M}\|\dot{\bar{q}}\|\|\bar{q}\|\left(\|\dot{\bar{q}}\|+\left\|\widetilde{q}_{m}\right\|+V_{M}\right)+\mu_{o}\left\|\dot{\widetilde{q}}_{m}\right\|^{2} \\
& +\gamma_{o}\left\|\dot{\widetilde{q}}_{s}\right\|^{2}+M_{s, m}^{-1} C_{s, M}\left(\left\|\dot{\widetilde{q}}_{s}\right\|+\gamma_{o}\left\|\widetilde{q}_{s}\right\|\right)\left(\left\|\dot{\widetilde{q}}_{s}\right\|+L_{p 1, M}\left\|\widetilde{q}_{s}\right\|\right)^{2}
\end{aligned}
$$

From the upperbound of $\beta\left(y, \dot{q}_{s}, \ddot{q}_{m}\right)$ (B.9), the upperbound of $\mu\left(\widetilde{q}_{m}\right), \gamma\left(\widetilde{q}_{s}\right)(3.31)$, and considering $y_{N}$ defined by (3.34), it follows that $\dot{V}(y)$ (B.1) can be upperbounded as in equation (3.35). 


\section{Appendix $\mathrm{C}$}

\section{Proof of Lemma 4.1}

In this appendix the synchronization closed loop error dynamics given by (4.23), (4.24), and (4.25) are developed.

From the definition of the synchronization errors (4.13) it follows that

$$
\dot{q}_{s}=\dot{e}+\dot{q}_{m}, \quad \ddot{q}_{s}=\ddot{e}+\ddot{q}_{m}, \quad q_{s}^{(3)}=e^{(3)}+q_{m}^{(3)}
$$

Consider a state space representation of the robot dynamics (4.1), (4.2), with states $q_{s}, \dot{q}_{s}, \theta_{s}, \dot{\theta}_{s}$, then from the nonlinear observer (4.20), (4.21) and the joint estimation errors (4.22), it follows that

$$
\begin{aligned}
& \dot{\tilde{q}}_{s}=\widetilde{\dot{q}}_{s}-\mu_{1} \widetilde{q}_{s} \\
& \dot{\tilde{q}}_{s}=-M_{s}^{-1}\left(q_{s}\right)\left(\left(2 C_{s}\left(q_{s}, \dot{q}_{s}\right)-C_{s}\left(q_{s}, \widetilde{\dot{q}}_{s}\right)\right) \widetilde{\dot{q}}_{s}+K_{s}\left(\widetilde{q}_{s}-\widetilde{\theta}_{s}\right)\right)-\mu_{2} \widetilde{q}_{s} \\
& \dot{\tilde{\theta}}_{s}=\widetilde{\dot{\theta}}_{s}-\mu_{3} \widetilde{q}_{s} \\
& \dot{\tilde{\theta}}_{s}=-J_{s}^{-1} K_{s}\left(\widetilde{\theta}_{s}-\widetilde{q}_{s}\right)-J_{s}^{-1} B_{v, s} \widetilde{\dot{\theta}}_{s}-\mu_{4} \widetilde{q}_{s}
\end{aligned}
$$

The first and third equation of (C.2) imply that

$$
\begin{array}{ll}
\tilde{\dot{q}}_{s}=\dot{\tilde{q}}_{s}+\mu_{1} \widetilde{q}_{s}, & \ddot{\widetilde{q}}_{s}=\dot{\tilde{\dot{q}}}_{s}-\mu_{1} \dot{\widetilde{q}}_{s} \\
\widetilde{\dot{\theta}}_{s}=\dot{\tilde{\theta}}_{s}+\mu_{3} \widetilde{q}_{s}, & \ddot{\tilde{\theta}}_{s}=\dot{\dot{\theta}}_{s}-\mu_{3} \dot{\tilde{q}}_{s}
\end{array}
$$

therefore (C.1) and (C.2) yield the joint estimation error dynamics (4.25).

On the other hand, consider the joint estimation errors given by (4.22), and introduce the variables $\widetilde{\ddot{q}}_{s}, \widetilde{q_{s}^{(3)}}$ as

$$
\widetilde{\ddot{q}}_{s}=\ddot{q}_{s}-\widehat{\ddot{q}}_{s}, \quad \widetilde{q_{s}^{(3)}}=q_{s}^{(3)}-\widehat{q_{s}^{(3)}}
$$

Differentiating (4.1) twice, and by considering (4.2), the control law $\tau_{s}$ (4.14), and property (2.26) it follows that

$$
M_{s}\left(q_{s}\right)\left(q_{s}^{(4)}-\widehat{v}(t)\right)+\Psi\left(q_{s}, \dot{q}_{s}, \ddot{q}_{s}, q_{s}^{(3)}, \widetilde{q}_{s}, \widetilde{\dot{q}}_{s}, \widetilde{\ddot{q}}_{s}, \widetilde{q_{s}^{(3)}}, \widetilde{\theta}_{s}, \widetilde{\dot{\theta}}_{s}\right)=0
$$

where $\Psi\left(q_{s}, \dot{q}_{s}, \ddot{q}_{s}, q_{s}^{(3)}, \widetilde{q}_{s}, \widetilde{\dot{q}}_{s}, \widetilde{\ddot{q}}_{s}, \widetilde{q_{s}^{(3)}}, \widetilde{\theta}_{s}, \widetilde{\dot{\theta}}_{s}\right)$ represents the mismatch with the desired linearization system, which is caused by absence of the high order derivatives of $q_{s}, q_{m}, \theta_{s}$. 
Notice that (C.6) depends on the signals $\dot{q}_{s}, \ddot{q}_{s}, q_{s}^{(3)}$ and $\widetilde{\ddot{q}}_{s}, \widetilde{q_{s}^{(3)}}$. Nevertheless this dependency can be changed in terms of the synchronization errors $\dot{e}, \ddot{e}, e^{(3)}$ and $\widetilde{q}_{s}, \dot{\widetilde{q}}_{s}$. According to the definition of $\widetilde{\ddot{q}}_{s}$ (C.5), and by considering (4.8), (4.15) and (C.3)

$$
\widetilde{\ddot{q}}_{s}=-M_{s}^{-1}\left(q_{s}\right)\left(\left(2 C_{s}\left(q_{s}, \dot{q}_{s}\right)-C_{s}\left(q_{s}, \dot{\vec{q}}_{s}+\mu_{1} \widetilde{q}_{s}\right)\right)\left(\dot{\tilde{q}}_{s}+\mu_{1} \widetilde{q}_{s}\right)+K_{s}\left(\widetilde{q}_{s}-\widetilde{\theta}_{s}\right)\right)
$$

In a similar way, but considering (C.5), (4.9) and (4.16), it follows that

$$
\begin{aligned}
\widetilde{q_{s}^{(3)}}= & -M_{s}^{-1}\left(q _ { s } \left(\dot{M}_{s}\left(q_{s}, \dot{q}_{s}\right) \ddot{q}_{s}-\dot{M}_{s}\left(q_{s}, \widehat{\dot{q}}_{s}\right) \widehat{\ddot{q}}_{s}+\dot{N}\left(q_{s}, \dot{q}_{s}, \ddot{q}_{s}\right)\right.\right. \\
& \left.-\dot{N}\left(q_{s}, \widehat{\dot{q}}_{s}, \widehat{\ddot{q}}_{s}\right)+K_{s}\left(\widetilde{\dot{q}}_{s}-\widetilde{\dot{\theta}}_{s}\right)\right)
\end{aligned}
$$

where, by considering property (2.26) and after a straightforward computation

$$
\begin{aligned}
& \dot{M}_{s}\left(q_{s}, \dot{q}_{s}\right) \ddot{q}_{s}-\dot{M}_{s}\left(q_{s}, \widehat{\dot{q}}_{s}\right) \widehat{\ddot{q}}_{s}=\frac{\partial M_{s}\left(q_{s}\right)}{\partial q_{s}}\left(\widetilde{\dot{q}}_{s} \ddot{q}_{s}+\left(\dot{q}_{s}-\widetilde{\dot{q}}_{s}\right) \widetilde{\ddot{q}}_{s}\right) \\
& \dot{N}\left(q_{s}, \dot{q}_{s}, \ddot{q}_{s}\right)-\dot{N}\left(q_{s}, \widehat{\dot{q}}_{s}, \widehat{\ddot{q}}_{s}\right)=C_{s}\left(q_{s}, \widetilde{\dot{q}}_{s}\right) \ddot{q}_{s}+\left(C_{s}\left(q_{s}, \dot{q}_{s}\right)-C_{s}\left(q_{s}, \widetilde{\dot{q}}_{s}\right)\right) \widetilde{\ddot{q}}_{s} \\
& +\frac{\partial g_{s}\left(q_{s}\right)}{\partial q_{s}} \widetilde{\dot{q}}_{s}+\left[\begin{array}{c}
C_{s 1}\left(q_{s}\right) \\
\vdots \\
C_{s n}\left(q_{s}\right)
\end{array}\right]\left(\widetilde{\ddot{q}}_{s} \dot{q}_{s}+\left(\ddot{q}_{s}-\widetilde{\ddot{q}}_{s}\right) \widetilde{\dot{q}}_{s}\right) \\
& +\dot{q}_{s} \frac{\partial}{\partial q_{s}}\left[\begin{array}{c}
C_{s 1}\left(q_{s}\right) \\
\vdots \\
C_{s n}\left(q_{s}\right)
\end{array}\right]\left(\widetilde{\dot{q}}_{s} \dot{q}_{s}+\left(\dot{q}_{s}-\widetilde{\dot{q}}_{s}\right) \widetilde{\dot{q}}_{s}\right)+ \\
& +\widetilde{\dot{q}}_{s} \frac{\partial}{\partial q_{s}}\left[\begin{array}{c}
C_{s 1}\left(q_{s}\right) \\
\vdots \\
C_{s n}\left(q_{s}\right)
\end{array}\right]\left(\left(\dot{q}_{s}-\widetilde{\dot{q}}_{s}\right)\left(\dot{q}_{s}-\widetilde{\dot{q}}_{s}\right)\right)
\end{aligned}
$$

Let $\Phi\left(q_{s}, \dot{q}_{m}, \ddot{q}_{m}, q_{m}^{(3)}, \dot{e}, \ddot{e}, e^{(3)}, \widetilde{q}_{s}, \dot{\widetilde{q}}_{s}, \widetilde{\theta}_{s}, \dot{\widetilde{\theta}}_{s}\right)$ denote the function $\Psi\left(q_{s}, \dot{q}_{s}, \ddot{q}_{s}, q_{s}^{(3)}, \widetilde{q}_{s}, \widetilde{\dot{q}}_{s}, \widetilde{\ddot{q}}_{s}, \widetilde{q_{s}^{(3)}}, \widetilde{\theta}_{s}, \widetilde{\dot{\theta}}_{s}\right)$ after substitution of the relations (C.3), (C.4), (C.7), (C.8), (C.9), (C.10), and (C.1), then

$$
\Phi\left(q_{s}, \dot{q}_{m}, \ddot{q}_{m}, q_{m}^{(3)}, \dot{e}, \ddot{e}, e^{(3)}, \widetilde{q}_{s}, \dot{\tilde{q}}_{s}, \widetilde{\theta}_{s}, \dot{\widetilde{\theta}}_{s}\right)=\Psi\left(q_{s}, \dot{q}_{s}, \ddot{q}_{s}, q_{s}^{(3)}, \widetilde{q}_{s}, \widetilde{\dot{q}}_{s}, \widetilde{\ddot{q}}_{s}, \widetilde{q_{s}^{(3)}}, \widetilde{\theta}_{s}, \widetilde{\dot{\theta}}_{s}\right)
$$

where $\Phi\left(q_{s}, \dot{q}_{m}, \ddot{q}_{m}, q_{m}^{(3)}, \dot{e}, \ddot{e}, e^{(3)}, \widetilde{q}_{s}, \dot{\widetilde{q}}_{s}, \widetilde{\theta}_{s}, \dot{\widetilde{\theta}}_{s}\right)$ is the result of a long, but straightforward, chain of substitutions and simplifications.

Substitution of (C.11) and (4.17) in (C.6), and taking into account (4.18), yields the synchronization error dynamics (4.23).

Consider the nonlinear observer (4.18) and the estimation synchronization errors (4.19), then it follows that

$$
\widehat{e}^{(4)}-\Gamma_{1} \widetilde{e}^{(3)}-\Gamma_{2} \ddot{\widetilde{e}}-\Gamma_{3} \dot{\tilde{e}}-\Gamma_{4} \widetilde{e}=0
$$

Subtraction of (C.12) from the synchronization error dynamics (4.23), and considering the estimation synchronization errors (4.19), yields the estimation synchronization error dynamics (4.24). 


\section{Appendix D}

\section{Proof of Lemma 4.7}

In this appendix we provide a general bound for the derivative of the Lyapunov matrix (4.35) along the synchronization closed loop error dynamics (4.26). Such derivative is given by (4.40).

Before getting the bound of (4.40) some bounds on the physical parameters are required.

From the properties of the matrices $M_{s}\left(q_{s}\right), C_{s}\left(q_{s}, \dot{q}_{s}\right) \dot{q}_{s}$, the gravity term $g_{s}\left(q_{s}\right)$, (see Section 2.2.3), and because their nonlinear terms contain only sinusoidal functions of $q_{s}$, we have that for all $q_{s} \in \mathbb{R}^{n}$, their partial derivatives can be bounded as

$$
\begin{gathered}
\left\|\frac{\partial M_{s}\left(q_{s}\right)}{\partial q_{s}}\right\| \leq M_{p M}, \quad\left\|\frac{\partial^{2} M_{s}\left(q_{s}\right)}{\partial q_{s}^{2}}\right\| \leq M_{p p M} \\
\left\|\frac{\partial g_{s}\left(q_{s}\right)}{\partial q_{s}}\right\| \leq G_{p M}, \quad\left\|\frac{\partial^{2} g_{s}\left(q_{s}\right)}{\partial q_{s}^{2}}\right\| \leq G_{p p M} \\
\left\|\left[\begin{array}{c}
C_{s 1}\left(q_{s}\right) \\
\vdots \\
C_{s n}\left(q_{s}\right)
\end{array}\right]\right\| \leq C_{q M}, \quad\left\|\frac{\partial}{\partial q_{s}}\left[\begin{array}{c}
C_{s 1}\left(q_{s}\right) \\
\vdots \\
C_{s n}\left(q_{s}\right)
\end{array}\right]\right\| \leq C_{p M} \\
\left\|\frac{\partial^{2}}{\partial q_{s}^{2}}\left[\begin{array}{c}
C_{s 1}\left(q_{s}\right) \\
\vdots \\
C_{s n}\left(q_{s}\right)
\end{array}\right]\right\| \leq C_{p p M}
\end{gathered}
$$

For the sake of simplicity and without loss of generality, assume that $Q_{x}=-\left(P_{x} A+\right.$ $\left.A^{T} P_{x}\right)$ is a symmetric positive definite block diagonal matrix, with $n \times n$ block entries, and denote the $i$-th diagonal $n \times n$ block of $Q_{x}$ by $Q_{x i}$. Then from the definition of $x_{N}, y_{N}$, and $\xi_{N}$ (4.44), and $\dot{V}$ given by (4.40), it follows that the term $x^{T} Q_{x} x$ in (4.40) can be bounded as $x_{N}^{T} R_{x} x_{N}$, with $R_{x} \in \mathbb{R}^{8 \times 8}$ given by

$$
R_{x}=\operatorname{diag}\left\{Q_{x i M}\right\} \quad i=1, \ldots, 8
$$

where $Q_{x i M}$ is the maximum eigenvalue of $Q_{x i}$, and such that positive definiteness of $Q_{x}$ implies that $R_{x}$ is positive definite.

On the other hand, from the matrix $Q_{y}$ (4.41), the term $\Omega$ (4.43), and by considering the bounds of $M_{s}\left(q_{s}\right), C_{s}\left(q_{s}, \dot{q}_{s}\right) \dot{q}_{s}, B_{v, s}$ and $g_{s}\left(q_{s}\right)$, and the partial derivatives of 
$M_{s}\left(q_{s}\right), C_{s}\left(q_{s}, \dot{q}_{s}\right)$, it follows that $y^{T} Q_{y} y$ can be bounded as $y_{N}^{T} R_{y} y_{N}$, with $R_{y} \in \mathbb{R}^{4 \times 4}$ given by

$$
\begin{aligned}
R_{y}=\left[\begin{array}{cccc}
\alpha_{0}^{*} & \alpha_{1}^{*} & \alpha_{2}^{*} & \alpha_{3}^{*} \\
\alpha_{1}^{*} & M_{s m} \mu_{1 m}-2 \lambda_{0}+2 C_{M} V_{M} & \eta_{0} \mu_{3 M}-\frac{1}{2} K_{s M} & \frac{1}{2} J_{s M} \mu_{3 M} \\
\alpha_{2}^{*} & \eta_{0} \mu_{3 M}-\frac{1}{2} K_{s M} & 2 \eta_{0} J_{s M}^{-1} K_{s m} & \alpha_{4}^{*} \\
\alpha_{3}^{*} & \frac{1}{2} J_{s M} \mu_{3 M} & \alpha_{4}^{*} & B_{v, s m}-4 \eta_{0}
\end{array}\right] \\
\alpha_{0}^{*}=2 \lambda_{0}\left(M_{s M}^{-1}\left(K_{s m}+2 C_{M} V_{M} \mu_{1 m}\right)+\mu_{2 m}\right) \\
\alpha_{1}^{*}=C_{M} V_{M}\left(2 M_{s m}^{-1} \lambda_{0}+\mu_{1 M}\right)-\frac{1}{2} M_{s M} \mu_{2 M}-\frac{1}{2} \beta_{1} \\
\alpha_{2}^{*}=-\lambda_{0} M_{s m}^{-1} K_{s M}+\eta_{0}\left(\mu_{4 M}+J_{s m}^{-1} B_{v, s M} \mu_{3 M}-J_{s m}^{-1} K_{s M}\right) \\
\alpha_{3}^{*}=\frac{1}{2}\left(B_{v, s M} \mu_{3 M}+J_{s M} \mu_{4 M}-K_{s M}\right) \\
\alpha_{4}^{*}=\eta_{0} J_{s m}^{-1} B_{v, s M}-\frac{1}{2} \mu_{4 M}-\frac{1}{2} \beta_{2}
\end{aligned}
$$

The matrices $R_{x}$ and $R_{y}$ are related to the bounds of the quadratic terms in $x$ and $y$ of $\dot{V}$ given by (4.40). Nevertheless, there exist bilinear cross terms in $x$ and $y$, coming from $Q_{x y}$ (4.42) and $\Omega$. These quadratic cross terms are bounded, such that, $R_{x y}$ corresponds to the bound of the bilinear cross terms of

$$
\begin{gathered}
\left\|x^{T} P_{x} B\left[-M_{s}^{-1}\left(\Phi+K_{s} J_{s}^{-1}\left(K_{s}\left(y_{1}-y_{3}\right)-B_{v, s}\left(y_{4}+\mu_{3} y_{1}\right)\right)\right)+q_{m}^{(4)}\right]\right\| \\
+\left\|x^{T} Q_{x y} x\right\|
\end{gathered}
$$

Consider $\Phi+K_{s} J_{s}^{-1} K_{s}\left(y_{1}-y_{3}\right)-K_{s} J_{s}^{-1} B_{v, s}\left(y_{4}+\mu_{3} y_{1}\right)$, which can be bounded as

$$
\left\|\Phi+K_{s} J_{s}^{-1} K_{s}\left(y_{1}-y_{3}\right)-K_{s} J_{s}^{-1} B_{v, s}\left(y_{4}+\mu_{3} y_{1}\right)\right\| \leq \Phi_{1}+\Phi_{r}
$$

where $\Phi_{1}$ contains terms of first order in $x, y$, and $\Phi_{r}$ contains the remaining terms (orders 2,3 and 4). After a long and straightforward computation it is obtained that $\Phi_{1}$ is given by

$$
\Phi_{1}=\left(\left(a_{1}+a_{3}\right) \mu_{1 M}+a_{2}+a_{3} \mu_{3 M}\right)\left\|y_{1}\right\|+\left(a_{1}+a_{3}\right)\left\|y_{2}\right\|+a_{2}\left\|y_{3}\right\|+a_{3}\left\|y_{4}\right\|
$$

with

$$
\begin{aligned}
a_{1}= & 2 M_{s m}^{-1} C_{M} V_{M}\left[A_{M}\left(M_{p M}+2 C_{q M}\right)+G_{p M}+K_{s M}\right. \\
& \left.+V_{M}^{2}\left(M_{p M}+2 C_{p M}\right)\right]+C_{p M} V_{M}\left(6 A_{M}+2 M_{s m}^{-1} C_{M} V_{M}^{2}\right)+4 C_{p p M} V_{M}^{3} \\
& +2\left(A_{M}^{2}+C_{p M} A_{M} V_{M}+2 M_{s m}^{-1} C_{p M} C_{M} V_{M}^{2}\right)+M_{s m}^{-1} V_{M}\left(2 M_{p M}+C_{M}+C_{q M}\right) \\
& \times\left[G_{p M}+3 C_{p M} V_{M}^{2}+\left(M_{p M}+C_{M}+C_{q M}\right)\left(A_{M}+2 M_{s m}^{-1} V_{M}^{2}\right)\right]+ \\
& +D_{M}\left(2 M_{p M}+C_{M}+C_{q M}\right)+2 M_{s m}^{-1} C_{M} V_{M} A_{M}\left(M_{p M}+2 C_{q M}\right) \\
& +2 G_{p p M}+2 M_{p p M} A_{M} V_{M} \\
& \\
a_{2}= & K_{s m} M_{s m}^{-1}\left\{2 A_{M}\left(M_{p M}+2 C_{q M}\right)+G_{p M}+K_{s M}+V_{M}^{2}\left(M_{p M}+2 C_{p M}\right)\right\} \\
& +K_{s m}\left[M_{s m}^{-1} C_{p M} V_{M}\left(2+V_{M}\right)\right. \\
& \left.+\left(M_{s m}^{-1} V_{M}\right)^{2}\left(M_{p M}+C_{M}+C_{q M}\right)\left(2 M_{p M}+C_{M}+C_{q M}\right)\right]
\end{aligned}
$$




$$
a_{3}=K_{s m} M_{s m}^{-1} V_{M}\left(2 M_{p M}+C_{M}+C_{q M}\right)
$$

Therefore, the matrix $R_{x y}$ is given by

$$
\begin{aligned}
& R_{x y}=M_{s m}^{-1} P_{Q}\left[\begin{array}{llll}
R_{1}^{*} & a_{1}+a_{3} & a_{2}+K_{s M}^{2} J_{s m}^{-1} & a_{3}+K_{s M} J_{s m}^{-1} B_{v, s M}
\end{array}\right] \\
& P_{Q}=\left[\begin{array}{c}
\left(P_{x 14}+P_{x 18}\right)_{M} \\
\left(P_{x 24}+P_{x 28}\right)_{M} \\
\left(P_{x 34}+P_{x 38}\right)_{M} \\
\left(P_{x 44}+P_{x 48}\right)_{M} \\
\left(P_{x 45}^{T}+P_{x 58}\right)_{M} \\
\left(P_{x 46}^{T}+P_{x 68}\right)_{M} \\
\left(P_{x 47}^{T}+P_{x 78}\right)_{M} \\
\left(P_{x 48}^{T}+P_{x 88}\right)_{M}
\end{array}\right] \\
& R_{1}^{*}=\left(a_{1}+a_{3}\right) \mu_{1 M}+a_{2}+a_{3} \mu_{3 M}+K_{s M} J_{s m}^{-1}\left(B_{v, s M} \mu_{3 M}-K_{s m}\right)
\end{aligned}
$$

Remark D.1 Notice that $a_{1}, a_{2}, a_{3}$ are uniquely determined by the physical parameters of the slave robot and the bounds of the master trajectories, and thus they do no depend on the control and observer gains. As a consequence $a_{1}, a_{2}, a_{3}$ must be evaluated only once and it can be done prior the gain tuning procedure.

At this point all the quadratic terms of $\dot{V}$ have been bounded in terms of $R_{x}, R_{y}, R_{x y}$, and $x_{N}, y_{N}, \xi_{N}$. Therefore it is only necessary to bound all the remaining terms originated from $\Omega$. From (4.43) and considering (D.3), it follows that

$$
\left\|\Omega\left(q_{s}, \dot{q}_{m}, \ddot{q}_{m}, q_{m}^{(3)}, q_{m}^{(4)}, x, y\right)\right\| \leq \Theta\left(V_{M}, A_{M}, D_{M}, E_{M}, \xi_{N}\right)
$$

where $\Theta\left(V_{M}, A_{M}, D_{M}, E_{M}, \xi_{N}\right)$ is given by

$$
\begin{aligned}
\Theta= & 2 x_{N}^{T} P_{Q}\left[M_{s m}^{-1} \Phi r+E_{M}\right]+C_{M} \mu_{1 M}\left\|y_{1}\right\|\left\|y_{2}\right\|^{2} \\
& +2 \lambda_{0} C_{M} M_{s m}^{-1}\left\|y_{1}\right\|\left\|y_{2}\right\|\left(\left\|y_{2}\right\|+\mu_{1 M}\left\|y_{1}\right\|\right) \\
& +C_{M}\left(\mu_{1 M}\left\|y_{1}\right\|+2\left\|x_{2}\right\|\right)\left(2 \lambda_{0} M_{s m}^{-1}\left\|y_{1}\right\|+\left\|y_{2}\right\|\right)\left(\left\|y_{2}\right\|+\mu_{1 M}\left\|y_{1}\right\|\right)
\end{aligned}
$$

with $P_{Q}$ as in (D.4). 


\section{Appendix E}

\section{Proof of Proposition 4.9}

In this appendix we provide conditions to ensure positive definiteness of the matrix $R_{y}$ given by (D.2), which is a necessary condition to prove positive definiteness of the matrix $R_{v}$ given by (4.46).

First, notice that the definition of $\beta_{1}, \beta_{2}$ given by (4.48), imply that $\alpha_{1}^{*}=0, \alpha_{4}^{*}=0$ in $R_{y}$ (D.2).

Second, let $R_{y i}$ denote the determinant of the $i$-th leading minor of $R_{y}$, then conditions for $R_{y i}>0, i=1, \ldots, 4$, are given by

- $R_{y 1}>0$ if $\lambda_{0}>0, \mu_{1 m}>0$, and $\mu_{2 m}>0$

- $R_{y 2}>0$ if $\mu_{1 m}>2 M_{s m}^{-1}\left(\lambda_{0}-C_{M} V_{M}\right)$

- For $R_{y 3}$, first notice that it can be written as $R_{y 3}=a_{31} \mu_{2 m}+a_{30}$, with $a_{31}=$ $b_{32} \mu_{3 M}^{2}+b_{31} \mu_{3 M}+b_{30}$, and $b_{32}<0$. Then $a_{31}>0$ if $\mu_{33_{-}}<\mu_{3 m}, \mu_{3 M}<\mu_{33}^{-}$, where

$$
\begin{aligned}
\mu_{33}- & =\frac{1}{4 J_{s M} \eta_{0}}\left(2 J_{s M} K_{s M}-4\left(2 J_{s M} \eta_{0} K_{s M}\left(M_{s m} \mu_{1 m}+2\left(C_{M} V_{M}-\lambda_{0}\right)\right)\right)^{1 / 2}\right) \\
\mu_{33}^{-} & =\frac{1}{4 J_{s M} \eta_{0}}\left(2 J_{s M} K_{s M}+4\left(2 J_{s M} \eta_{0} K_{s M}\left(M_{s m} \mu_{1 m}+2\left(C_{M} V_{M}-\lambda_{0}\right)\right)\right)^{1 / 2}\right)
\end{aligned}
$$

because $a_{31}>0$, then $\mu_{2 m}>-a_{31}^{-1} a_{30}$ implies $R_{y 3}>0$.

- $R_{y 4}$ can be written as $R_{y 4}=a_{41} \mu_{2 m}+a_{40}$, with $a_{41}=b_{42} \mu_{3 M}^{2}+b_{41} \mu_{3 M}+b_{40}$, and $b_{42}<0$ if $\eta_{0}$ holds

$$
0<\eta_{0}<\min \left\{\frac{B_{v, s m}}{4}, \frac{1}{8 J_{s M}}\left(J_{s M} B_{v, s m}+\left(J_{s M}^{2} B_{v, s m}^{2}+8 J_{s M} J_{s m}^{2} K_{s m}\right)^{1 / 2}\right)\right\}
$$

Then $b_{42}<0$ implies that $a_{41}>0$ if $\mu_{34_{-}}<\mu_{3 m}, \mu_{3 M}<\mu_{34}^{-}$, where

$$
\begin{array}{r}
\mu_{34_{-}}=\frac{1}{2\left(\eta_{0}\left(2 J_{s M}\left(4 \eta_{0}-B_{v, s m}\right)\right)-J_{s m}^{2} K_{s m}\right)}\left(2 J_{s M} K_{s m} \eta_{0}\left(4 \eta_{0}-B_{v, s m}\right)\right. \\
+\left(2 J_{s M} K_{s m} \eta_{0}\right)^{1 / 2}\left(( 4 \eta _ { 0 } - B _ { v , s m } ) \left[J_{s m}^{2} K_{s M}^{2}\right.\right. \\
\left.\left.+8 \eta_{0}\left(2 \lambda_{0}-2 C_{M} V_{M}-M_{s m} \mu_{1 m}\right)\left(J_{s M}^{-1} J_{s m}^{2} K_{s m}-2 \eta_{0}\left(4 \eta_{0}-B_{v, s m}\right)\right]\right)^{1 / 2}\right)
\end{array}
$$




$$
\begin{array}{r}
\mu_{34}^{-}=\frac{1}{2\left(\eta_{0}\left(2 J_{s M}\left(4 \eta_{0}-B_{v, s m}\right)\right)-J_{s m}^{2} K_{s m}\right)}\left(2 J_{s M} K_{s m} \eta_{0}\left(4 \eta_{0}-B_{v, s m}\right)\right. \\
-\left(2 J_{s M} K_{s m} \eta_{0}\right)^{1 / 2}\left(( 4 \eta _ { 0 } - B _ { v , s m } ) \left[J_{s m}^{2} K_{s M}^{2}\right.\right. \\
\left.\left.+8 \eta_{0}\left(2 \lambda_{0}-2 C_{M} V_{M}-M_{s m} \mu_{1 m}\right)\left(J_{s M}^{-1} J_{s m}^{2} K_{s m}-2 \eta_{0}\left(4 \eta_{0}-B_{v, s m}\right)\right]\right)^{1 / 2}\right)
\end{array}
$$

because $a_{41}>0$, then $\mu_{2 m}>-a_{41}^{-1} a_{40}$ implies $R_{y 4}>0$.

If the above conditions are satisfied, then the determinants of all the leading minors of $R_{y}$ are positive. Therefore by Sylvester's criterion it follows that $R_{y}$ is positive definite. 


\section{Appendix F}

\section{Proof of Lemma 5.9}

In this appendix we develop the synchronization closed loop error dynamics of the multi-composed system of $p$ robots in closed loop with the controller (5.21) and the observers (5.26). First the error dynamics for the observers (5.26) is obtained, then the coupled synchronization error dynamics is formulated. Through the formulation of the error dynamics, Assumptions 5.1 and 5.8 are considered.

\section{Observer error dynamics}

Let $x_{i, 1}=q_{i}$ and $x_{i, 2}=\dot{q}_{i}$ define states for the $i$-th robot dynamics given by (5.1). Then from a state space representation of (5.1), with states $x_{i, 1}$ and $x_{i, 2}$, and the observer defined by (5.26), it follows that after substitution of the control $\tau_{i}$ given by $(5.21)$

$$
\begin{aligned}
\frac{d}{d t} \widetilde{q}_{i} & =\widetilde{\dot{q}}_{i}-\mu_{1} \widetilde{q}_{i} \\
\frac{d}{d t} \widetilde{\dot{q}}_{i} & =M_{i}\left(q_{i}\right)^{-1}\left[C_{i}\left(q_{i}, \widehat{\dot{q}}_{i}\right) \widehat{\dot{q}}_{i}-C_{i}\left(q_{i}, \dot{q}_{i}\right) \dot{q}_{i}\right]-\mu_{2} \widetilde{q}_{i}
\end{aligned}
$$

From the definition of the estimation errors (5.27) and the properties of the Coriolis term, Section 2.2.3, it is obtained that

$$
C_{i}\left(q_{i}, \widehat{\dot{q}}_{i}\right) \widehat{\dot{q}}_{i}-C_{i}\left(q_{i}, \dot{q}_{i}\right) \dot{q}_{i}=C_{i}\left(q_{i}, \widetilde{\dot{q}}_{i}\right)\left(\widetilde{\dot{q}}_{i}-2 \dot{q}_{i}\right)
$$

The partial synchronization errors (5.9) imply that $\dot{q}_{i}=\dot{q}_{d}+\dot{e}_{i, i}$. Therefore after a simple computation is it obtained that the observer error dynamics is given by

$$
\ddot{\widetilde{q}}_{i}=M_{i}\left(q_{i}\right)^{-1} C_{i}\left(q_{i}, \dot{\widetilde{q}}_{i}+\mu_{1} \widetilde{q}_{i}\right)\left(\dot{\widetilde{q}}_{i}+\mu_{1} \widetilde{q}_{i}-2 \dot{q}_{d}-2 \dot{e}_{i, i}\right)-\mu_{1} \dot{\widetilde{q}}_{i}-\mu_{2} \widetilde{q}_{i}
$$

\section{Synchronization error dynamics}

From the dynamics of the robots (5.1) and the synchronization controller (5.21) it follows that

$$
M_{i}\left(q_{i}\right)\left(\ddot{q}_{i}-\widehat{\ddot{q}}_{r i}\right)+C_{i}\left(q_{i}, \dot{q}_{i}\right) \dot{q}_{i}-C_{i}\left(q_{i}, \widehat{\dot{q}}_{i}\right) \widehat{\dot{q}}_{r i}=-K_{d, i} \widehat{\dot{s}}_{i}-K_{p, i} s_{i} \quad i=1, \ldots, p
$$


On the one hand, from the definition of the estimate of the reference signal $\widehat{\ddot{q}}_{r i}$ given by (5.23), the partial synchronization errors (5.8) and the estimation errors (5.27), it is obtained that

$$
\ddot{q}_{i}-\widehat{\ddot{q}}_{r i}=\ddot{e}_{i, i}+\sum_{j=1, j \neq i}^{p} K_{i, j} \ddot{e}_{i, j}-\sum_{j=1, j \neq i}^{p} K_{i, j}\left(\widetilde{\ddot{q}}_{i}-\widetilde{\ddot{q}}_{j}\right)
$$

and from the observer error dynamics (F.1) it follows that

$$
\begin{aligned}
\widetilde{\ddot{q}}_{i}-\widetilde{\ddot{q}}_{j}= & M_{i}\left(q_{i}\right)^{-1} C_{i}\left(q_{i}, \dot{\widetilde{q}}_{i}+\mu_{1} \widetilde{q}_{i}\right)\left(\dot{\widetilde{q}}_{i}+\mu_{1} \widetilde{q}_{i}-2 \dot{q}_{d}-2 \dot{e}_{i, i}\right)-\mu_{2} \widetilde{q}_{i} \\
& -M_{j}\left(q_{j}\right)^{-1} C_{j}\left(q_{j}, \dot{\widetilde{q}}_{j}+\mu_{1} \widetilde{q}_{j}\right)\left(\dot{\widetilde{q}}_{j}+\mu_{1} \widetilde{q}_{j}-2 \dot{q}_{d}-2 \dot{e}_{j, j}\right)+\mu_{2} \widetilde{q}_{j}
\end{aligned}
$$

In a similar way, but from the definition of $\widehat{\dot{q}}_{r i}$ given by (5.22), it follows that

$$
\widehat{\dot{s}}_{i}:=\dot{e}_{i, i}+\sum_{j=1, j \neq i}^{p} K_{i, j} \dot{e}_{i, j}-\dot{\widetilde{q}}_{i}-\mu_{1} \widetilde{q}_{i}+\sum_{j=1, j \neq i}^{p} K_{i, j}\left(-\dot{\widetilde{q}}_{i}-\mu_{1} \widetilde{q}_{i}+\dot{\widetilde{q}}_{j}+\mu_{1} \widetilde{q}_{j}\right)
$$

On the other hand from the properties of the Coriolis term, Section 2.2.3, the definition of $\widehat{\dot{q}}_{r i}$ given by (5.22) and the observer error dynamics (F.1) it follows that

$$
\begin{aligned}
C_{i}\left(q_{i}, \dot{q}_{i}\right) \dot{q}_{i}-C_{i}\left(q_{i}, \hat{\dot{q}}_{i}\right) \widehat{\dot{q}}_{r i}=C_{i}\left(q_{i}, \dot{e}_{i, i}+\dot{q}_{d}\right)\left(\dot{e}_{i, i}+\sum_{j=1, j \neq i}^{p} K_{i, j} \dot{e}_{i, j}\right) \\
+C_{i}\left(q_{i}, \dot{e}_{i, i}+\dot{q}_{d}-\dot{\widetilde{q}}_{i}-\mu_{1} \widetilde{q}_{i}\right) \sum_{j=1, j \neq i}^{p} K_{i, j}\left(\dot{\widetilde{q}}_{i}+\mu_{1} \widetilde{q}_{i}-\dot{\widetilde{q}}_{j}-\mu_{1} \widetilde{q}_{j}\right) \\
+C_{i}\left(q_{i}, \dot{\widetilde{q}}_{i}+\mu_{1} \widetilde{q}_{i}\right)\left(\dot{q}_{d}-\sum_{j=1, j \neq i}^{p} K_{i, j} \dot{e}_{i, j}\right)
\end{aligned}
$$

From equations (F.2) and (F.3) it follows that the synchronization closed loop error dynamics are given by $($ for $i=1, \ldots, p)$

$$
\begin{gathered}
\ddot{\widetilde{q}}_{i}=M_{i}\left(q_{i}\right)^{-1} C_{i}\left(q_{i}, \dot{\widetilde{q}}_{i}+\mu_{1} \widetilde{q}_{i}\right)\left(\dot{\widetilde{q}}_{i}+\mu_{1} \widetilde{q}_{i}-2 \dot{q}_{d}-2 \dot{e}_{i, i}\right)-\mu_{1} \dot{\widetilde{q}}_{i}-\mu_{2} \widetilde{q}_{i} \\
M_{i}\left(q_{i}\right)\left(\ddot{e}_{i, i}+\sum_{j=1, j \neq i}^{p} K_{i, j} \ddot{e}_{i, j}\right)=M_{i}\left(q_{i}\right) \sum_{j=1, j \neq i}^{p} K_{i, j}\left(\widetilde{\ddot{q}}_{i}-\widetilde{\ddot{q}}_{j}\right) \\
-\left(C_{i}\left(q_{i}, \dot{q}_{i}\right) \dot{q}_{i}-C_{i}\left(q_{i}, \widehat{\dot{q}}_{i}\right) \widehat{\dot{q}}_{r i}\right)-K_{d, i} \widehat{\dot{s}}_{i}-K_{p, i} s_{i}
\end{gathered}
$$

Substitution of equations (F.4 - F.7) in (F.9), and considering the synchronization errors $s_{i}, \dot{s}_{i}$, defined by (5.6), results in the synchronization error dynamics (5.30, 5.31). 


\section{Appendix G}

\section{Proof of Theorem 5.11}

In this appendix we present the details behind the proof of the Theorem 5.11

Time derivative of the Lyapunov function $V(\dot{s}, \dot{\widetilde{q}}, s, \widetilde{q})$

The time derivative of the Lyapunov function (5.37) along the error dynamics (5.30, $5.31)$ is given by

$$
\dot{V}(\dot{s}, \dot{\widetilde{q}}, s, \widetilde{q})=\sum_{i=1}^{p} \dot{V}_{i}\left(\dot{s}_{i}, \dot{\widetilde{q}}_{i}, s_{i}, \widetilde{q}_{i}\right)=\sum_{i=1}^{p}\left\{\dot{V}_{i, 1}\left(\dot{s}_{i}, s_{i}\right)+\dot{V}_{i, 2}\left(\dot{\tilde{q}_{i}}, \widetilde{q}_{i}\right)\right\}
$$

where according to (5.39) and (5.40)

$$
\begin{aligned}
\dot{V}_{i, 1}\left(\dot{s}_{i}, s_{i}\right)= & \dot{s}_{i}^{T} K_{p, i} s_{i}+\dot{s}_{i}^{T} M_{i}\left(q_{i}\right) \ddot{s}_{i}+\frac{1}{2} \dot{s}_{i}^{T} \dot{M}_{i}\left(q_{i}\right) \dot{s}_{i} \\
\dot{V}_{i, 2}\left(\dot{\widetilde{q}}_{i}, \widetilde{q}_{i}\right)= & \left(\dot{\widetilde{q}}_{i}^{T} M_{i}\left(q_{i}\right)+\eta_{i}\left(\widetilde{q}_{i}\right) \widetilde{q}_{i}^{T}\right) \ddot{\widetilde{q}}_{i}+\frac{1}{2} \dot{\widetilde{q}_{i}^{T}} \dot{M}_{i}\left(q_{i}\right) \dot{\widetilde{q}}_{i} \\
& +\eta_{i}\left({ }_{i}\right) \dot{\widetilde{q}_{i}^{T}} \widetilde{q}_{i}+\dot{\eta}_{i}\left(\widetilde{q}_{i}\right) \dot{\tilde{q}_{i}^{T}} \widetilde{q}_{i}+\widetilde{q}_{i}^{T}\left(\mu_{2}+\beta_{i} I_{n}\right) \dot{\widetilde{q}}_{i}
\end{aligned}
$$

From the synchronization closed loop error dynamics $(5.30,5.31)$, and by the properties of the Coriolis term, it follows that

$$
\begin{aligned}
& \dot{V}_{i, 1}\left(\dot{s}_{i}, s_{i}\right)=-\dot{s}_{i}^{T} K_{d, i} \dot{s}_{i}-\dot{s}_{i}^{T} C_{i}\left(q_{i}, \dot{\widetilde{q}}_{i}+\mu_{1} \widetilde{q}_{i}\right)\left(\dot{q}_{d}-\sum_{j=1, j \neq i}^{p} K_{i, j} \dot{e}_{i, j}\right) \\
& -\dot{s}_{i}^{T} C_{i}\left(q_{i}, \dot{e}_{i, i}+\dot{q}_{d}-\dot{\widetilde{q}}_{i}-\mu_{1} \widetilde{q}_{i}\right) \sum_{j=1, j \neq i}^{p} K_{i, j}\left(\dot{\widetilde{q}}_{i}+\mu_{1} \widetilde{q}_{i}-\dot{\widetilde{q}}_{j}-\mu_{1} \widetilde{q}_{j}\right) \\
& +\dot{s}_{i}^{T} K_{d, i}\left(\dot{\widetilde{q}}_{i}+\mu_{1} \widetilde{q}_{i}+\sum_{j=1, j \neq i}^{p} K_{i, j}\left(\dot{\widetilde{q}}_{i}+\mu_{1} \widetilde{q}_{i}-\dot{\widetilde{q}}_{j}-\mu_{1} \widetilde{q}_{j}\right)\right)+\dot{s}_{i}^{T} M_{i}\left(q_{i}\right) \times \\
& \times \sum_{j=1, j \neq i}^{p} K_{i, j}\left[M_{i}\left(q_{i}\right)^{-1} C_{i}\left(q_{i}, \dot{\widetilde{q}}_{i}+\mu_{1} \widetilde{q}_{i}\right)\left(\dot{\widetilde{q}}_{i}+\mu_{1} \widetilde{q}_{i}-2 \dot{q}_{d}-2 \dot{e}_{i, i}\right)-\mu_{1} \dot{\widetilde{q}}_{i}-\mu_{2} \widetilde{q}_{i}\right. \\
& \left.+\mu_{1} \dot{\widetilde{q}}_{j}+\mu_{2} \widetilde{q}_{j}-M_{j}\left(q_{j}\right)^{-1} C_{j}\left(q_{j}, \dot{\widetilde{q}}_{j}+\mu_{1} \widetilde{q}_{j}\right)\left(\dot{\widetilde{q}}_{j}+\mu_{1} \widetilde{q}_{j}-2 \dot{q}_{d}-2 \dot{e}_{j, j}\right)\right]
\end{aligned}
$$


$\dot{V}_{i, 2}\left(\dot{\widetilde{q}}_{i}, \widetilde{q}_{i}\right)=\left(M_{i}\left(q_{i}\right)^{-1} C_{i}\left(q_{i}, \dot{\widetilde{q}}_{i}+\mu_{1} \widetilde{q}_{i}\right)\left(\dot{\tilde{q}}_{i}+\mu_{1} \widetilde{q}_{i}-2 \dot{q}_{d}-2 \dot{e}_{i, i}\right)-\mu_{1} \dot{\widetilde{q}}_{i}-\mu_{2} \widetilde{q}\right) \times$
$\times\left(\dot{\widetilde{q}}_{i}^{T} M_{i}\left(q_{i}\right)+\eta_{i}\left(\widetilde{q}_{i}\right) \widetilde{q}_{i}^{T}\right)+\frac{1}{2} \dot{\tilde{q}}_{i}^{T} \dot{M}_{i}\left(q_{i}\right) \dot{\widetilde{q}}_{i}+\eta_{i}\left({ }_{i}\right) \dot{q}_{i}^{T} \dot{\tilde{q}}_{i}+\dot{\eta}_{i}\left(\widetilde{q}_{i}\right) \dot{q}_{i}^{T} \widetilde{q}_{i}+\widetilde{q}_{i}^{T}\left(\mu_{2}+\beta_{i} I_{n}\right) \dot{\widetilde{q}}_{i}$

After a long but straightforward computation and by considering Assumptions 5.1, $5.8,5.10$, the properties of the robot dynamics, and the bound of $\dot{M}_{i}\left(q_{i}\right)$ given by

$$
M_{i, p m}\left\|\dot{q}_{i}\right\| \leq\left\|\dot{M}_{i}\left(q_{i}\right)\right\| \leq M_{i, p M}\left\|\dot{q}_{i}\right\|
$$

it follows that $\dot{V}_{i}\left(\dot{s}_{i}, \dot{\widetilde{q}}_{i}, s_{i}, \widetilde{q}_{i}\right)$ in (G.1) is bounded as

$$
\begin{aligned}
& \dot{V}_{i}\left(\dot{s}_{i}, \dot{\widetilde{q}}_{i}, s_{i}, \widetilde{q}_{i}\right) \leq-\left(M_{i, m} \mu_{1}-2 \eta_{0}-\frac{1}{2} M_{i, p M}+2 V_{M} C_{i, M}\right)\left\|\dot{\widetilde{q}}_{i}\right\|^{2} \\
& -K_{d i}\left\|\dot{s}_{i}\right\|^{2}-\eta_{0}\left(\mu_{2}+2 V_{M} C_{i, M} M_{i, m}^{-1} \mu_{1}\right)\left\|\widetilde{q}_{i}\right\|^{2} \\
& +\left(\beta_{i}-\eta_{0} \mu_{1}-2 V_{M} C_{i, M}\left(\mu_{1}+\eta_{0} M_{i, m}^{-1}\right)+\mu_{2}\left(1-M_{i, m}\right)\right)\left\|\dot{\widetilde{q}}_{i}\right\|\left\|\widetilde{q}_{i}\right\| \\
& +\left[K_{d i}-V_{M} C_{i, M}+\sum_{j=1, j \neq i}^{p} K_{i, j}\left(K_{d i}-M_{i, m} \mu_{1}-3 V_{M} C_{i, M}\right)\right]\left\|\dot{s}_{i}\right\|\left\|\dot{\widetilde{q}}_{i}\right\| \\
& +\left[\mu_{1}\left(K_{d i}-V_{M} C_{i, M}\right)+\sum_{j=1, j \neq i}^{p} K_{i, j}\left(K_{d i} \mu_{1}-M_{i, m} \mu_{2}-3 V_{M} C_{i, M} \mu_{1}\right)\right]\left\|\dot{s}_{i}\right\|\left\|\widetilde{q}_{i}\right\| \\
& +\left(M_{i, m} \mu_{1}-K_{d i}+V_{M} C_{i, M}\right)\left\|\dot{s}_{i}\right\| \sum_{j=1, j \neq i}^{p} K_{i, j}\left\|\dot{\widetilde{q}}_{j}\right\|+2 V_{M} M_{i, m}\left\|\dot{s}_{i}\right\| \times \\
& \times \sum_{j=1, j \neq i}^{p} K_{i, j}\left(M_{j, m}^{-1} C_{j, M}\left\|\dot{\widetilde{q}}_{j}\right\|\right)+2 \mu_{1} V_{M} M_{i, m}\left\|\dot{s}_{i}\right\| \sum_{j=1, j \neq i}^{p} K_{i, j}\left(M_{j, m}^{-1} C_{j, M}\left\|\widetilde{q}_{j}\right\|\right) \\
& +\left(M_{i, m} \mu_{2}-K_{d i} \mu_{1}+\mu_{1} V_{M} C_{i, M}\right)\left\|\dot{s}_{i}\right\| \sum_{j=1, j \neq i}^{p} K_{i, j}\left\|\widetilde{q}_{j}\right\|+\Phi_{3, i}
\end{aligned}
$$

with $\Phi_{3, i}$ given by

$$
\begin{aligned}
& \Phi_{3, i}=C_{i, M}\left\|\dot{s}_{i}\right\|\left(\left\|\dot{\widetilde{q}}_{i}\right\|+\mu_{1}\left\|\widetilde{q}_{i}\right\|\right)\left(\sum_{j=1, j \neq i}^{p} K_{i, j}\left\|\dot{e}_{i, j}\right\|\right)+\left(\left\|\dot{\widetilde{q}}_{i}\right\|+\eta_{0} M_{i, m}^{-1}\left\|\widetilde{q}_{i}\right\|\right) \times \\
& \times C_{i, M}\left(\left\|\dot{\widetilde{q}}_{i}\right\|+\mu_{1}\left\|\widetilde{q}_{i}\right\|\right)\left(\left\|\dot{\widetilde{q}}_{i}\right\|+\mu_{1}\left\|\widetilde{q}_{i}\right\|-2\left\|\dot{e}_{i, i}\right\|\right)-\left(\left\|\dot{e}_{i, i}\right\|-\left\|\dot{\widetilde{q}}_{i}\right\|-\mu_{1}\left\|\widetilde{q}_{i}\right\|\right) \times \\
& \times C_{i, M}\left\|\dot{s}_{i}\right\| \sum_{j=1, j \neq i}^{p} K_{i, j}\left(\left\|\dot{\tilde{q}}_{i}\right\|-\left\|\dot{\widetilde{q}}_{j}\right\|+\mu_{1}\left(\left\|\widetilde{q}_{i}\right\|-\left\|\widetilde{q}_{j}\right\|\right)\right)+M_{i, m}\left\|\dot{s}_{i}\right\| \times \\
& \times \sum_{j=1, j \neq i}^{p} K_{i, j}\left(M_{i, m}^{-1} C_{i, M}\left(\left\|\dot{\tilde{q}}_{i}\right\|+\mu_{1}\left\|\widetilde{q}_{i}\right\|\right)\left(\left\|\dot{\widetilde{q}}_{i}\right\|+\mu_{1}\left\|\widetilde{q}_{i}\right\|-2\left\|\dot{e}_{i, i}\right\|\right)\right. \\
& \left.-M_{j, m}^{-1} C_{j, M}\left(\left\|\dot{\widetilde{q}}_{j}\right\|+\mu_{1}\left\|\widetilde{q}_{j}\right\|\right)\left(\left\|\dot{\widetilde{q}}_{j}\right\|+\mu_{1}\left\|\widetilde{q}_{j}\right\|-2\left\|\dot{e}_{j, j}\right\|\right)\right)
\end{aligned}
$$

From the definition of $\beta_{i}$ in $(5.42)$, the coefficient of the bilinear term $\left\|\dot{\widetilde{q}}_{i}\right\|\left\|\widetilde{q}_{i}\right\|$ in (G.4) is equal to zero. Therefore from $\widetilde{q}$ in (5.36) the bound (G.4) results in (5.46). 


\section{Appendix $\mathbf{H}$}

\section{Dynamic model of the CFT robot}

Here the dynamic model and kinematic relations of the CFT transposer robots in the multi-robot system are presented. The multi-robot system, formed by two CFT robots, is installed in the Dynamics and Control Technology Laboratory of the Department of Mechanical Engineering at the Eindhoven University of Technology. The individual robots in the multi-composed system are referred to as robot 1 (R1) and robot 2 (R2).

The robot has four degrees of freedom, which are depicted in Figure 6.1. A schematic representation of the robot in the Cartesian space is given in Figure H.1. The translational movement correspond to the degree of freedom of the body attached to frame $\{1\}$. The rotational base corresponds to the body attached to frame $\{2\}$. The up and down, forward and backward movements correspond to the degrees of freedom of the origin of the frame $\{7\}$, denoted by $P_{e}$. The point $P_{e}$ is the point where the passively actuated tool is connected.

The transposer robot is a Cartesian robot, but the synchronization controllers proposed in Chapters 3 and 5 are designed in the joint space. Therefore the first step for implementing the controllers is to relate the Cartesian and joint spaces. For this a direct kinematic model in both spaces is required. The kinematic model of the robot can be formulated based on the Denavit-Hartenberg parameters (Craig 1988), while the dynamic model can be formulated based on Denavit-Hartenberg parameters and the Euler-Lagrange approach (Spong and Vidyasagar 1989), (Lewis et al. 1993). A detailed formulation of the kinematic and dynamic model of the CFT transposer robot can be found in the technical report (Rodriguez-Angeles et al. 2002a).

\section{Direct kinematics in the Cartesian space}

Consider Figure H.1 and denote the 4 Cartesian degrees of freedom of the robot as $x_{c 1}, x_{c 2}, x_{c 3}$ and $x_{c 4}$, such that $x_{c 1}, x_{c 2}$ correspond to the up and down, forward and backward movement of the arm respectively, and $x_{c 3}, x_{c 4}$ are the rotation and translation of the base in which the arm is mounted.

Figure H.1 shows a schematic diagram of the robot, $x_{c 3}, x_{c 4}$ are absolute coordinates and are referred with respect to an inertial frame - frame $\{0\}$ - at the base of the 


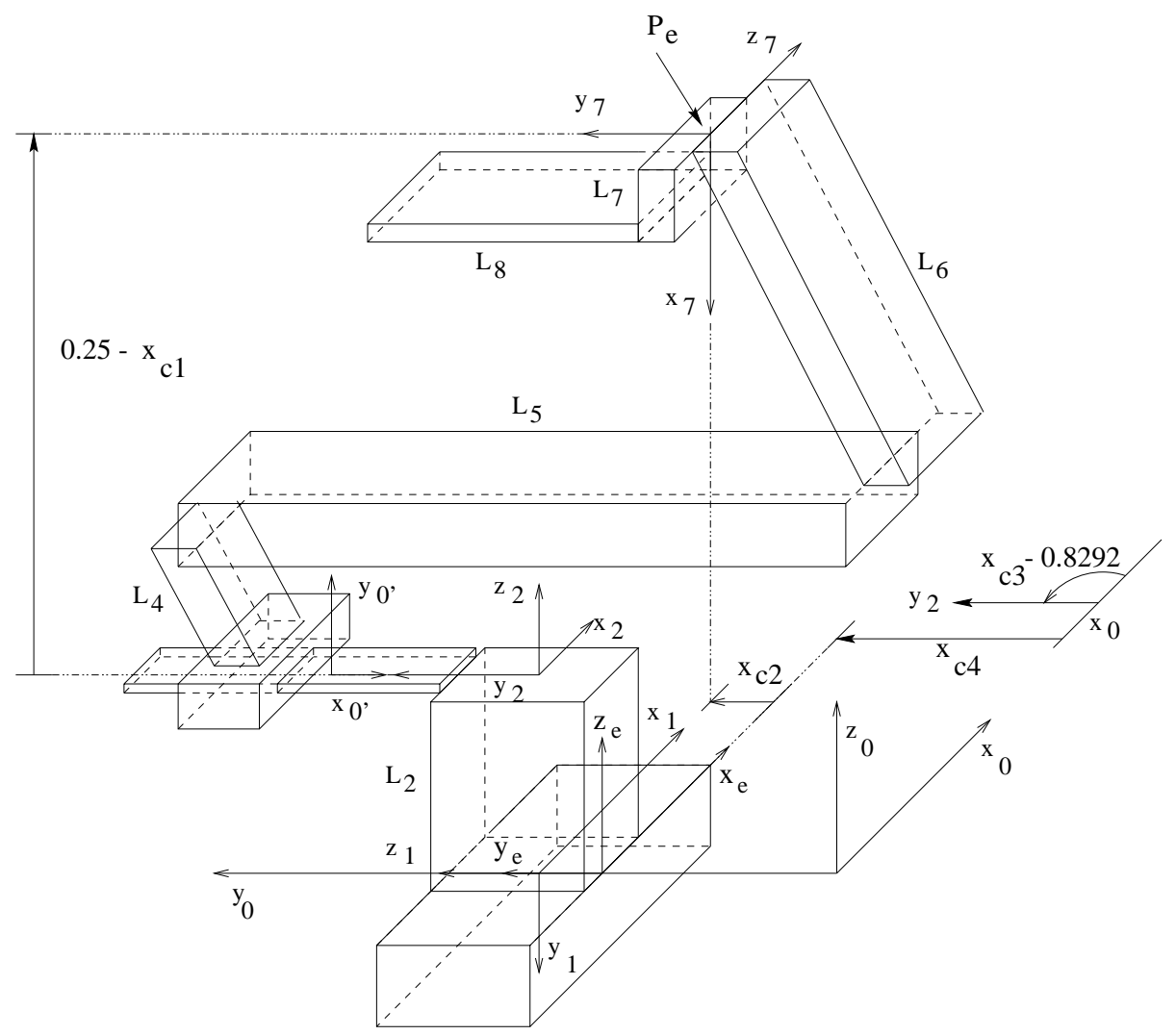

Figure H.1: CFT-transposer robot: Cartesian coordinates

robot. The coordinates $x_{c 1}, x_{c 2}$ are relative coordinates and are referred with respect to a frame at the edge of the translational platform - frame $\{e\} . x_{c 2}$ is defined under the consideration that the upper arm is aligned with the $y_{0}$ axis. $x_{c 4}$ is the distance from the origin of frame $\{0\}$ to the origin of frame $\{e\}$.

The physical dimensions of the CFT-robot are listed in Table H.1, $d_{i_{-} i+1}$ denotes the distance between the origin of frame $\{i\}$ and $\{i+1\}, d_{s}$ is the distance between the origin of frame $\{1\}$ and $\{e\}$, and $L_{i}$ denotes the length of the $i$-th link.

\begin{tabular}{|c|c|c|c|}
\hline Dimension & Value $[\mathrm{m}]$ & Dimension & Value $[\mathrm{m}]$ \\
\hline$L_{2}, d_{1 \_2}$ & 0.25 & $L_{8}$ & 0.48 \\
\hline$L_{4}$ & 0.05 & $d_{4}, d_{5}$ & 0.0 \\
\hline$L_{5}$ & 0.35 & $d_{6}$ & 0.04 \\
\hline$L_{6}$ & 0.30 & $d_{s}$ & 0.185 \\
\hline$L_{7}$ & 0.08 & $d_{2 \_0^{\prime}}$ & 0.0916 \\
\hline
\end{tabular}

Table H.1: Dimensions of the robot.

Consider the point $P_{e}$ as the origin of frame $\{7\}$, then the coordinates of $P_{e}$ with respect to the frame $\{0\}$ are given by

$$
x_{P_{e}, 0}=\left(x_{c 2}-d_{s}\right) \cos \left(x_{c 3}-0.8292\right)
$$




$$
\begin{aligned}
& y_{p_{e}, 0}=x_{c 4}+d_{s}+\left(x_{c 2}-d_{s}\right) \sin \left(x_{c 3}-0.8292\right) \\
& z_{p_{e}, 0}=L_{2}+0.25-x_{c 1}
\end{aligned}
$$

As mentioned the passively actuated tool, that is attached to the end of the upper arm is constrained to remain horizontal at all time. Therefore the coordinates of the tip of the tool $\left(x_{T}, y_{T}, z_{T}\right)$ with respect to the frame $\{0\}$ are given by

$$
\begin{aligned}
& x_{T}=\left(x_{c 2}-d_{s}+L_{8}\right) \cos \left(x_{c 3}-0.8292\right) \\
& y_{T}=x_{c 4}+d_{s}+\left(x_{c 2}-d_{s}+L_{8}\right) \sin \left(x_{c 3}-0.8292\right) \\
& z_{T}=L_{2}+0.25-x_{c 1}-L_{7}
\end{aligned}
$$

Equations (H.2) correspond to the direct kinematics of the robot and determine any position of the tip of the tool in the robot working space as function of the robot coordinates $x_{c 1}, x_{c 2}, x_{c 3}$ and $x_{c 4}$.

\section{Direct kinematics in the joint space}

To define the direct kinematics of the robot, the-Denavit Hartenberg parameters are considered, in particular the convention presented in (Craig 1988) is used. According to (Craig 1988) the frames can be assigned as in Figure H.2, with the resulting Denavit-Hartenberg parameters listed in Table H.2. $L_{i}$ is the length of link $i, d_{i}$ is the offset of each link along the $z_{i}$-axis, $q_{1}, q_{3}$ are the translations along $z_{1}, z_{3}$ respectively, and for $i=2,4,5,6,7, q_{i}$ is the rotation angle about the $z_{i}$-axis. All the dimensions of the robot are listed in Table H.1.

\begin{tabular}{|c|c|c|c|c|}
\hline$i$ & $a_{i}$ & $\alpha_{i}$ & $d_{i}$ & $q_{i}$ \\
\hline 0 & 0 & $-\frac{\pi}{2}$ & - & - \\
\hline 1 & 0 & $\frac{\pi}{2}$ & $q_{1}$ & 0 \\
\hline 2 & 0 & $-\frac{\pi}{2}$ & $L_{2}$ & $q_{2}$ \\
\hline 3 & 0 & $-\frac{\pi}{2}$ & $q_{3}$ & $-\frac{\pi}{2}$ \\
\hline 4 & $L_{4}$ & 0 & 0 & $q_{4}$ \\
\hline 5 & $L_{5}$ & 0 & 0 & $q_{5}$ \\
\hline 6 & $L_{6}$ & 0 & $d_{6}$ & $q_{6}$ \\
\hline 7 & $L_{7}$ & 0 & $d_{7}$ & $q_{7}$ \\
\hline 8 & $L_{8}$ & 0 & 0 & $\frac{\pi}{2}$ \\
\hline 9 & - & - & 0 & 0 \\
\hline
\end{tabular}

Table H.2: Denavit-Hartenberg parameters for the CFT-robot

The Denavit-Hartenberg parameters listed in Table H.2 and the frames in Figure H.2 account for 7 joints, i.e. $q_{i}, i=1, \ldots, 7$. However, by construction the joints $q_{3}, q_{6}$, and $q_{7}$ are kinematically constrained as function of $q_{4}, q_{5}$ as follows

$$
\begin{gathered}
q_{3}=L_{4}\left(\cos \left(-q_{4}-q_{5}+\frac{\pi}{2}\right)+\cos \left(-q_{4}+\frac{\pi}{2}\right)\right)+d_{2 \_} 0^{\prime} \\
q_{6}=-q_{5} \\
q_{7}=\pi-q_{4}
\end{gathered}
$$




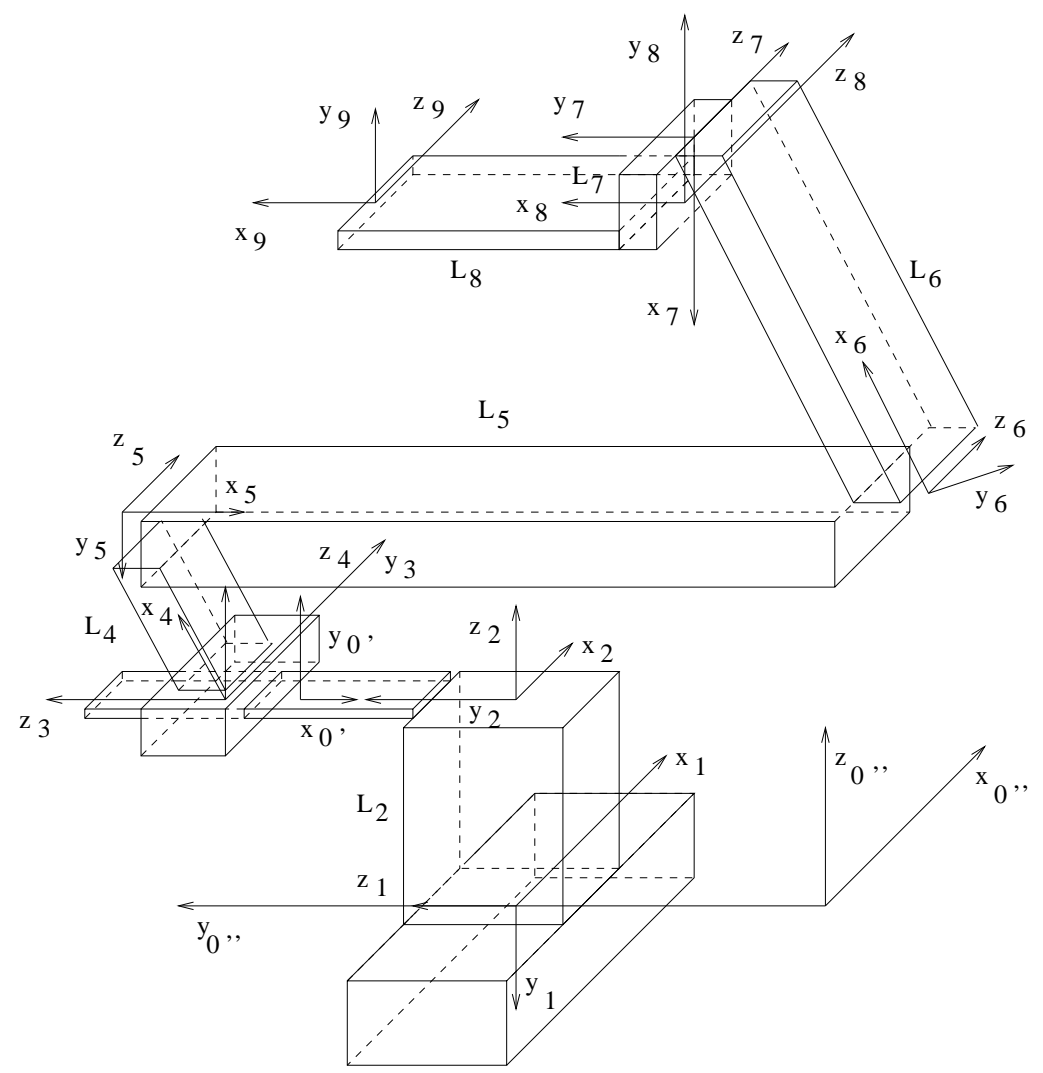

Figure H.2: Frames for the CFT-transposer robot

According to (Craig 1988) and by considering the Denavit-Hartenberg parameters (Table H.2), and the kinematic constraints (H.3 - H.5), it follows that the direct kinematics between the tip position of the tool $\left(x_{T}, y_{T}, z_{T}\right)$ and the actuated joints $q_{1}, q_{2}, q_{4}$, and $q_{5}$ is given by

$$
\begin{aligned}
x_{T}= & -\left(L_{8}+d_{20^{\prime}}\right) \sin \left(q_{2}\right)+\frac{1}{2}\left[\cos \left(-q_{2}+q_{4}\right)-\cos \left(q_{2}+q_{4}\right)\right] L_{6} \\
& +\frac{1}{2}\left[\cos \left(q_{5}-q_{2}+q_{4}\right)-\cos \left(q_{5}+q_{2}+q_{4}\right)\right]\left(L_{5}-L_{4}\right) \\
y_{T}= & q_{1}+\left(L_{8}+d_{2 \_} 0^{\prime}\right) \cos \left(q_{2}\right)-\frac{1}{2}\left[\sin \left(q_{2}+q_{4}\right)-\sin \left(q_{4}-q_{2}\right)\right] L_{6} \\
& -\frac{1}{2}\left[\sin \left(q_{5}-q_{2}+q_{4}\right)+\sin \left(q_{5}+q_{2}+q_{4}\right)\right]\left(L_{5}-L_{4}\right) \\
z_{T}= & L_{2}-L_{7}+\left(L_{6}+L_{4}\right) \cos \left(q_{4}\right)+L_{5} \cos \left(q_{4}+q_{5}\right)
\end{aligned}
$$

Since the direct kinematics (H.2) and (H.6) represent the same point in the space with respect to frame $\{0\}$, it follows that there exists a unique relation between the Cartesian coordinates $x_{c 1}, x_{c 2}, x_{c 3}$ and $x_{c 4}$ and the joint coordinates $q_{1}, q_{2}, q_{4}$, and $q_{5}$.

From the geometry of the robot it follows that the Cartesian coordinates $x_{c 1}, x_{c 2}, x_{c 3}$ 
and $x_{c 4}$ and the joint coordinates $q_{1}, q_{2}, q_{4}$, and $q_{5}$ are related as

$$
\begin{aligned}
& x_{c 1}=0.25-\left(L_{4}+L_{6}\right)\left(\cos \left(q_{4}+q_{5}\right)+\cos \left(q_{4}\right)\right) \\
& x_{c 2}=d_{2 \_} 0^{\prime}-d_{s}-L_{6}\left(\sin \left(q_{4}+q_{5}\right)+\sin \left(q_{4}\right)\right) \\
& x_{c 3}=q_{2}+2.4 \\
& x_{c 4}=q_{1}-d_{s}
\end{aligned}
$$

The relation (H.7) defines the direct kinematics between Cartesian coordinates and joint coordinates. It is important since it allows to transform quantities and variables between the Cartesian and the joint spaces, for instance, forces and torques via the Jacobian of (H.7). A particular use of (H.7) is the design of desired trajectories in the joint space. Note that equations (H.2) and (H.6) represent the same point in the space with respect to frame $\{0\}$. But for any given desired trajectory for the tip of the robot, denoted by $\left(x_{T, d}(t), y_{T, d}(t), z_{T, d}(t)\right)$, it is easier to solve (H.2) than (H.6). Then the desired tip trajectory is transformed in a desired trajectory in terms of the Cartesian coordinates $x_{c 1}, x_{c 2}, x_{c 3}$ and $x_{c 4}$, and by the inverse of (H.7) it is transformed in a desired trajectory for the joint coordinates $q_{1}, q_{2}, q_{4}$, and $q_{5}$. This sequence of transformations is used in the experiments in order to generate joint space desired trajectories for the proposed synchronization schemes.

\section{Joint space dynamics}

The dynamics of the CFT transposer robots are given by $(6.1,6.2)$, i.e.

$$
\begin{gathered}
M\left(q_{i}\right) \ddot{q}_{i}+C\left(q_{i}, \dot{q}_{i}\right) \dot{q}_{i}+g\left(q_{i}\right)+f\left(\dot{q}_{i}\right)=\tau_{i}, \quad i=1,2 \\
f\left(\dot{q}_{i}\right)=B_{v, i} \dot{q}_{i}+B_{f 1, i}\left(1-\frac{2}{1+e^{2 w_{1, i} \dot{q}_{i}}}\right)+B_{f 2, i}\left(1-\frac{2}{1+e^{2 w_{2, i} \dot{q}_{i}}}\right)
\end{gathered}
$$

The entries of the matrices $M\left(q_{i}\right), C\left(q_{i}, \dot{q}_{i}\right)$ and the gravity vector $g\left(q_{i}\right)$ can be computed one by one from the Denavit-Hartenberg parameters (Table H.2), see (Lee 1982), (Rodriguez-Angeles et al. 2002a). In this section the entries of the dynamics of the CFT robot are given, and a set of estimated physical parameters for robot R1 and R2 are listed in Table H.3.

Since the robots R1 and R2 in the multi-composed system are structurally identical, they are described by the same dynamic model, but they differ in the physical parameters. The dynamic model of the transposer robot (H.8, H.9) includes 32 physical parameters, denoted by $\theta_{i}, j, i=1,2, j=1, \ldots, 32$, where $i$ identifies the robot and $j$ the parameter. A detailed parametrization of the dynamics (H.8, H.9) and physical interpretation of the parameters $\theta_{i},{ }_{j}$ can be found in (Rodriguez-Angeles et al. 2002a).

\section{Entries of the inertia matrix $M\left(q_{i}\right)$}

The entries of the symmetric inertia matrix $M\left(q_{i}\right) \in \mathbb{R}^{4 \times 4}$, as function of the generalized coordinates $q_{i}=\left[\begin{array}{llll}q_{i, 1} & q_{i, 2} & q_{i, 4} & q_{i, 5}\end{array}\right]^{T}$ of robot $i, i=1,2$, and the parameters $\theta_{i}, j, j=1, \ldots 32$ are given by

$$
M_{1,1}=\theta_{i, 1}+\theta_{i, 11}+\theta_{i, 12}
$$




$$
\begin{aligned}
& M_{1,2}=\left(-\theta_{i, 12} d_{2 \_0^{\prime}}-\theta_{i, 11} d_{2 \_0^{\prime}}-\theta_{i, 3}\right) \sin \left(q_{i, 2}\right)+\left(\theta_{i, 2}+d_{6} \theta_{i, 11}\right) \cos \left(q_{i, 2}\right) \\
& +\frac{1}{2}\left(\left(L_{4}-L_{5}\right)\left(\theta_{i, 12}+\theta_{i, 11}\right)-\theta_{i, 9}\right)\left(\cos \left(q_{i, 5}+q_{i, 2}+q_{i, 4}\right)\right. \\
& \left.-\cos \left(q_{i, 5}-q_{i, 2}+q_{i, 4}\right)\right) \\
& +\frac{1}{2}\left(\theta_{i, 7}+\theta_{i, 5}+\theta_{i, 12} L_{6}\right)\left(\cos \left(-q_{i, 2}+q_{i, 4}\right)-\cos \left(q_{i, 2}+q_{i, 4}\right)\right) \\
& +\frac{1}{2}\left(\theta_{i, 8}+\theta_{i, 6}\right)\left(\sin \left(q_{i, 2}+q_{i, 4}\right)-\sin \left(-q_{i, 2}+q_{i, 4}\right)\right) \\
& +\frac{1}{2}\left(-\sin \left(q_{i, 5}-q_{i, 2}+q_{i, 4}\right)+\sin \left(q_{i, 5}+q_{i, 2}+q_{i, 4}\right)\right) \theta_{i, 10} \\
& M_{1,3}=\frac{1}{2}\left(-\theta_{i, 5}-\theta_{i, 7}-\theta_{i, 12} L_{6}\right)\left(\cos \left(q_{i, 2}+q_{i, 4}\right)+\cos \left(-q_{i, 2}+q_{i, 4}\right)\right) \\
& +\frac{1}{2}\left(\left(L_{4}-L_{5}\right)\left(\theta_{i, 12}+\theta_{i, 11}\right)-\theta_{i, 9}\right)\left(\cos \left(q_{i, 5}-q_{i, 2}+q_{i, 4}\right)\right. \\
& \left.+\cos \left(q_{i, 5}+q_{i, 2}+q_{i, 4}\right)\right) \\
& +\frac{1}{2}\left(\theta_{i, 8}+\theta_{i, 6}\right)\left(\sin \left(q_{i, 2}+q_{i, 4}\right)+\sin \left(-q_{i, 2}+q_{i, 4}\right)\right) \\
& +\frac{1}{2}\left(\sin \left(q_{i, 5}+q_{i, 2}+q_{i, 4}\right)+\sin \left(q_{i, 5}-q_{i, 2}+q_{i, 4}\right)\right) \theta_{i, 10} \\
& M_{1,4}=\frac{1}{2}\left(\left(L_{4}-L_{5}\right)\left(\theta_{i, 11}+\theta_{i, 12}\right)-\theta_{i, 9}\right)\left(\cos \left(q_{i, 5}+q_{i, 2}+q_{i, 4}\right)\right. \\
& \left.+\cos \left(q_{i, 5}-q_{i, 2}+q_{i, 4}\right)\right) \\
& +\frac{1}{2}\left(\sin \left(q_{i, 5}+q_{i, 2}+q_{i, 4}\right)+\sin \left(q_{i, 5}-q_{i, 2}+q_{i, 4}\right)\right) \theta_{i, 10} \\
& M_{2,2}=\left(\left(L_{5}-L_{4}\right)\left(\sin \left(q_{i, 5}\right)+\sin \left(q_{i, 5}+2 q_{i, 4}\right)\right)-2 \cos \left(q_{i, 4}\right) d_{2 \_0^{\prime}}\right) \theta_{i, 8}+\theta_{i, 4} \\
& +\left(-2 d_{2 \_0^{\prime}} \cos \left(q_{i, 4}+q_{i, 5}\right)-L_{4} \sin \left(2 q_{i, 5}+2 q_{i, 4}\right)\right) \theta_{i, 10}+\theta_{i, 12} d_{2 \_0^{\prime}}^{2} \\
& +\left(\left(\frac{1}{2}-\frac{1}{2} \cos \left(2 q_{i, 5}+2 q_{i, 4}\right)\right)\left(L_{5}^{2}+L_{4}^{2}\right)+2 L_{4} d_{2 \_} 0^{\prime} \sin \left(q_{i, 4}+q_{i, 5}\right)+d_{6}^{2}\right. \\
& \left.+d_{2 \_0^{\prime}}^{2}+\left(\left(\cos \left(2 q_{i, 5}+2 q_{i, 4}\right)-1\right) L_{4}-2 d_{2 \_0^{\prime}} \sin \left(q_{i, 4}+q_{i, 5}\right)\right) L_{5}\right) \theta_{i, 11} \\
& +\left(\left(\cos \left(2 q_{i, 4}\right)-\cos \left(q_{i, 5}\right)-1+\cos \left(q_{i, 5}+2 q_{i, 4}\right)\right) L_{4}\right. \\
& \left.-2 d_{2 \_0^{\prime}} \sin \left(q_{i, 4}\right)\right) \theta_{i, 5}-2\left(\sin \left(q_{i, 4}+q_{i, 5}\right) L_{5}+\sin \left(q_{i, 4}\right) L_{6}\right) d_{2 \_0^{\prime}} \theta_{i, 12} \\
& +\left(\left(\cos \left(q_{i, 5}+2 q_{i, 4}\right)-\cos \left(q_{i, 5}\right)\right)\left(L_{4}-L_{5}\right)-2 d_{2_{-} 0^{\prime}} \sin \left(q_{i, 4}\right)\right) \theta_{i, 7} \\
& -\frac{1}{2} \theta_{i, 12}\left(\cos \left(2 q_{i, 4}\right)-1\right) L_{6}^{2}-\frac{1}{2}\left(\cos \left(2 q_{i, 5}+2 q_{i, 4}\right)-1\right)\left(L_{5}^{2}+L_{4}^{2}\right) \theta_{i, 12} \\
& +\left(-L_{4}\left(\sin \left(2 q_{i, 4}\right)+\sin \left(q_{i, 5}+2 q_{i, 4}\right)+\sin \left(q_{i, 5}\right)\right)-2 \cos \left(q_{i, 4}\right) d_{2 \_0^{\prime}}\right) \theta_{i, 6} \\
& +\left(\left(\cos \left(2 q_{i, 5}+2 q_{i, 4}\right)-1\right) L_{4}+\left(\cos \left(q_{i, 5}\right)-\cos \left(q_{i, 5}+2 q_{i, 4}\right)\right) L_{6}\right) L_{5} \theta_{i, 12} \\
& +\left(\left(\cos \left(2 q_{i, 5}+2 q_{i, 4}\right)-1\right) L_{4}-2 d_{2 \_} 0^{\prime} \sin \left(q_{i, 4}+q_{i, 5}\right)\right) \theta_{i, 9} \\
& +\left(2 \sin \left(q_{i, 4}+q_{i, 5}\right) d_{2{ }^{\prime} 0^{\prime}}-\left(\cos \left(q_{i, 5}\right)+\cos \left(q_{i, 5}+2 q_{i, 4}\right)\right) L_{6}\right) L_{4} \theta_{i, 12} \\
& M_{2,3}=-\theta_{i, 7} d_{6} \cos \left(q_{i, 4}\right)+\theta_{i, 8} d_{6} \sin \left(q_{i, 4}\right)+\theta_{i, 11} d_{6}\left(L_{4}-L_{5}\right) \cos \left(q_{i, 4}+q_{i, 5}\right) \\
& M_{2,4}=\theta_{i, 11} d_{6}\left(L_{4}-L_{5}\right) \cos \left(q_{i, 4}+q_{i, 5}\right)
\end{aligned}
$$




$$
\begin{aligned}
& M_{3,3}=\left(\left(\left(L_{5}-L_{6}\right) L_{4}+2 L_{5} L_{6}\right) \theta_{i, 12}+L_{5} L_{4} \theta_{i, 11}+\left(\theta_{i, 9}-\theta_{i, 5}-\theta_{i, 7}\right) L_{4}\right. \\
& \left.+2 \theta_{i, 7} L_{5}\right) \cos \left(q_{i, 5}\right)+L_{4}\left(\theta_{i, 6}+\theta_{i, 8}\right) \sin \left(2 q_{i, 4}\right) \\
& -L_{4}\left(\left(\frac{1}{2} L_{4}+L_{6}\right) \theta_{i, 12}+\frac{1}{2} L_{4} \theta_{i, 11}+\theta_{i, 5}+\theta_{i, 7}\right) \cos \left(2 q_{i, 4}\right) \\
& -L_{4}\left(\left(L_{6}+L_{5}\right) \theta_{i, 12}+L_{5} \theta_{i, 11}+\theta_{i, 9}+\theta_{i, 7}+\theta_{i, 5}\right) \cos \left(q_{i, 5}+2 q_{i, 4}\right) \\
& +\left(\theta_{i, 8}+\theta_{i, 10}+\theta_{i, 6}\right) L_{4} \sin \left(q_{i, 5}+2 q_{i, 4}\right)+\left(\left(2 L_{5}-L_{4}\right) \theta_{i, 8}\right. \\
& \left.-\left(\theta_{i, 6}+\theta_{i, 10}\right) L_{4}\right) \sin \left(q_{i, 5}\right)+\left(L_{4}^{2}+\left(L_{6}-L_{5}\right) L_{4}+L_{5}^{2}+L_{6}^{2}\right) \theta_{i, 12} \\
& +\left(\left(\frac{1}{2} L_{4}-L_{5}\right)\left(\theta_{i, 12}+\theta_{i, 11}\right)-\theta_{i, 9}\right) L_{4} \cos \left(2 q_{i, 5}+2 q_{i, 4}\right) \\
& +\left(L_{5}^{2}-L_{5} L_{4}+L_{4}^{2}\right) \theta_{i, 11}+\left(\theta_{i, 7}-\theta_{i, 9}+\sin \left(2 q_{i, 5}+2 q_{i, 4}\right) \theta_{i, 10}-\theta_{i, 5}\right) L_{4} \\
& M_{3,4}=\frac{1}{2}\left(\sin \left(q_{i, 5}+2 q_{i, 4}\right)-\sin \left(q_{i, 5}\right)\right) L_{4} \theta_{i, 6}+\frac{1}{2} \theta_{i, 12}\left(\cos \left(2 q_{i, 5}+2 q_{i, 4}\right)+1\right) L_{4}^{2} \\
& +\left(\frac{1}{2} \cos \left(q_{i, 5}\right)-\cos \left(2 q_{i, 5}+2 q_{i, 4}\right)-\frac{1}{2} \cos \left(q_{i, 5}+2 q_{i, 4}\right)-1\right) L_{4} \theta_{i, 9} \\
& +\left(\frac{1}{2} \sin \left(q_{i, 5}+2 q_{i, 4}\right)+\sin \left(2 q_{i, 5}+2 q_{i, 4}\right)-\frac{1}{2} \sin \left(q_{i, 5}\right)\right) L_{4} \theta_{i, 10} \\
& +\left(L_{5} \cos \left(q_{i, 5}\right)-\frac{1}{2}\left(\cos \left(q_{i, 5}\right)+\cos \left(q_{i, 5}+2 q_{i, 4}\right)\right) L_{4}\right) \theta_{i, 7} \\
& +\left(\left(L_{5}-\frac{1}{2} L_{4}\right) \sin \left(q_{i, 5}\right)+\frac{1}{2} L_{4} \sin \left(q_{i, 5}+2 q_{i, 4}\right)\right) \theta_{i, 8}+\theta_{i, 12} L_{5}^{2} \\
& +\left(L_{5}^{2}+\frac{1}{2}\left(\cos \left(q_{i, 5}\right)-\cos \left(q_{i, 5}+2 q_{i, 4}\right)\right) L_{4} L_{5}+\frac{1}{2}\left(1+\cos \left(2 q_{i, 5}+2 q_{i, 4}\right)\right)\right. \\
& \left.\times\left(L_{4}^{2}-2 L_{4} L_{5}\right)\right) \theta_{i, 11}-\frac{1}{2}\left(\cos \left(q_{i, 5}\right)+\cos \left(q_{i, 5}+2 q_{i, 4}\right)\right) L_{4} \theta_{i, 5} \\
& +\left(\cos \left(q_{i, 5}\right) L_{6}-\frac{1}{2}\left(2+2 \cos \left(2 q_{i, 5}+2 q_{i, 4}\right)+\cos \left(q_{i, 5}+2 q_{i, 4}\right)\right.\right. \\
& \left.\left.-\cos \left(q_{i, 5}\right)\right) L_{4}\right) L_{5} \theta_{i, 12}-\frac{1}{2} \theta_{i, 12}\left(\cos \left(q_{i, 5}\right)+\cos \left(q_{i, 5}+2 q_{i, 4}\right)\right) L_{6} L_{4} \\
& M_{4,4}=\left(\left(\frac{1}{2} L_{4}^{2}-L_{5} L_{4}\right)\left(\theta_{i, 12}+\theta_{i, 11}\right)-L_{4} \theta_{i, 9}\right) \cos \left(2 q_{i, 5}+2 q_{i, 4}\right) \\
& +\left(\frac{1}{2} L_{4}^{2}-L_{5} L_{4}+L_{5}^{2}\right)\left(\theta_{i, 12}+\theta_{i, 11}\right)+\left(\sin \left(2 q_{i, 5}+2 q_{i, 4}\right) \theta_{i, 10}-\theta_{i, 9}\right) L_{4}
\end{aligned}
$$

\section{Entries of the Coriolis matrix $C\left(q_{i}, \dot{q}_{i}\right)$}

The entries of the Coriolis matrix $C\left(q_{i}, \dot{q}_{i}\right) \in \mathbb{R}^{4 \times 4}$, as function of the generalized coordinates $q_{i}=\left[\begin{array}{llll}q_{i, 1} & q_{i, 2} & q_{i, 4} & q_{i, 5}\end{array}\right]^{T}$ of robot $i, i=1,2$, and the parameters $\theta_{i, j}, j=1, \ldots 32$ are given by

$$
\begin{gathered}
C_{1,1}=C_{2,1}=C_{3,1}=C_{4,1}=0 \\
C_{1,2}=\frac{1}{2}\left(\theta_{i, 7}+\theta_{i, 5}+L_{6} \theta_{i, 12}\right)\left(\left(\dot{q}_{i, 2}-\dot{q}_{i, 4}\right) \sin \left(q_{i, 4}-q_{i, 2}\right)+\left(\dot{q}_{i, 2}+\dot{q}_{i, 4}\right)\right. \\
\left.\times \sin \left(q_{i, 2}+q_{i, 4}\right)\right)-\left(\left(\theta_{i, 12}+\theta_{i, 11}\right) d_{2 \_0^{\prime}}+\theta_{i, 3}\right) \dot{q}_{i, 2} \cos \left(q_{i, 2}\right)
\end{gathered}
$$




$$
\begin{aligned}
& +\frac{1}{2}\left(\theta_{i, 9}+\left(L_{5}-L_{4}\right)\left(\theta_{i, 12}+\theta_{i, 11}\right)\right)\left(\left(\dot{q}_{i, 4}+\dot{q}_{i, 2}+\dot{q}_{i, 5}\right)\right. \\
& \left.\times \sin \left(q_{i, 5}+q_{i, 2}+q_{i, 4}\right)+\left(\dot{q}_{i, 2}-\dot{q}_{i, 4}-\dot{q}_{i, 5}\right) \sin \left(q_{i, 5}-q_{i, 2}+q_{i, 4}\right)\right) \\
& -\left(\theta_{i, 2}+d_{6} \theta_{i, 11}\right) \dot{q}_{i, 2} \sin \left(q_{i, 2}\right)+\frac{1}{2}\left(\theta_{i, 8}+\theta_{i, 6}\right)\left(\left(\dot{q}_{i, 2}-\dot{q}_{i, 4}\right) \cos \left(q_{i, 4}-q_{i, 2}\right)\right. \\
& \left.+\left(\dot{q}_{i, 2}+\dot{q}_{i, 4}\right) \cos \left(q_{i, 2}+q_{i, 4}\right)\right)+\frac{1}{2}\left(\left(\dot{q}_{i, 2}-\dot{q}_{i, 4}-\dot{q}_{i, 5}\right)\right. \\
& \left.\times \cos \left(q_{i, 5}-q_{i, 2}+q_{i, 4}\right)+\left(\dot{q}_{i, 4}+\dot{q}_{i, 2}+\dot{q}_{i, 5}\right) \cos \left(q_{i, 5}+q_{i, 2}+q_{i, 4}\right)\right) \theta_{i, 10} \\
& C_{1,3}=\frac{1}{2}\left(\theta_{i, 8}+\theta_{i, 6}\right)\left(\left(\dot{q}_{i, 4}-\dot{q}_{i, 2}\right) \cos \left(q_{i, 4}-q_{i, 2}\right)+\left(\dot{q}_{i, 2}+\dot{q}_{i, 4}\right) \cos \left(q_{i, 2}+q_{i, 4}\right)\right) \\
& +\frac{1}{2}\left(\theta_{i, 9}+\left(L_{5}-L_{4}\right)\left(\theta_{i, 12}+\theta_{i, 11}\right)\right)\left(\left(\dot{q}_{i, 4}+\dot{q}_{i, 2}+\dot{q}_{i, 5}\right)\right. \\
& \left.\times \sin \left(q_{i, 5}+q_{i, 2}+q_{i, 4}\right)+\left(\dot{q}_{i, 4}-\dot{q}_{i, 2}+\dot{q}_{i, 5}\right) \sin \left(q_{i, 5}-q_{i, 2}+q_{i, 4}\right)\right) \\
& +\frac{1}{2}\left(\theta_{i, 5}+\theta_{i, 7}+L_{6} \theta_{i, 12}\right)\left(\left(\dot{q}_{i, 4}-\dot{q}_{i, 2}\right) \sin \left(q_{i, 4}-q_{i, 2}\right)+\left(\dot{q}_{i, 2}+\dot{q}_{i, 4}\right)\right. \\
& \left.\times \sin \left(q_{i, 2}+q_{i, 4}\right)\right)+\frac{1}{2}\left(\left(\dot{q}_{i, 4}+\dot{q}_{i, 2}+\dot{q}_{i, 5}\right) \cos \left(q_{i, 5}+q_{i, 2}+q_{i, 4}\right)\right. \\
& \left.+\left(\dot{q}_{i, 4}+\dot{q}_{i, 5}-\dot{q}_{i, 2}\right) \cos \left(q_{i, 5}-q_{i, 2}+q_{i, 4}\right)\right) \theta_{i, 10} \\
& C_{1,4}=\frac{1}{2}\left(\left(\dot{q}_{i, 4}+\dot{q}_{i, 2}+\dot{q}_{i, 5}\right) \cos \left(q_{i, 5}+q_{i, 2}+q_{i, 4}\right)+\left(\dot{q}_{i, 4}-\dot{q}_{i, 2}+\dot{q}_{i, 5}\right)\right. \\
& \left.\times \cos \left(q_{i, 5}-q_{i, 2}+q_{i, 4}\right)\right) \theta_{i, 10}+\frac{1}{2}\left(\theta_{i, 9}+\left(L_{5}-L_{4}\right)\left(\theta_{i, 12}+\theta_{i, 11}\right)\right) \\
& \times\left(\left(\dot{q}_{i, 4}+\dot{q}_{i, 2}+\dot{q}_{i, 5}\right) \sin \left(q_{i, 5}+q_{i, 2}+q_{i, 4}\right)\right. \\
& \left.+\left(\dot{q}_{i, 4}+\dot{q}_{i, 5}-\dot{q}_{i, 2}\right) \sin \left(q_{i, 5}-q_{i, 2}+q_{i, 4}\right)\right) \\
& C_{2,2}=-\frac{1}{2}\left(L_{4} \theta_{i, 6}-L_{5} \theta_{i, 8}+\theta_{i, 8} L_{4}\right)\left(\left(2 \dot{q}_{i, 4}+\dot{q}_{i, 5}\right) \cos \left(2 q_{i, 4}+q_{i, 5}\right)\right. \\
& \left.+\dot{q}_{i, 5} \cos \left(q_{i, 5}\right)\right)-\dot{q}_{i, 4} d_{2_{-} 0^{\prime}}\left(\theta_{i, 5}+\theta_{i, 7}+L_{6} \theta_{i, 12}\right) \cos \left(q_{i, 4}\right) \\
& -\left(\dot{q}_{i, 4}+\dot{q}_{i, 5}\right)\left(\theta_{i, 9}+\left(L_{5}-L_{4}\right)\left(\theta_{i, 12}+\theta_{i, 11}\right)\right) d_{2 \_0^{\prime}} \cos \left(q_{i, 4}+q_{i, 5}\right) \\
& -\frac{1}{2}\left(\dot{q}_{i, 4}+\dot{q}_{i, 5}\right)\left(2 L_{4} \theta_{i, 9}-\left(L_{5}-L_{4}\right)^{2}\left(\theta_{i, 12}+\theta_{i, 11}\right)\right) \sin \left(2 q_{i, 5}+2 q_{i, 4}\right) \\
& -\frac{1}{2} \dot{q}_{i, 4}\left(2 L_{4} \theta_{i, 5}-L_{6}^{2} \theta_{i, 12}\right) \sin \left(2 q_{i, 4}\right)-\dot{q}_{i, 4} L_{4} \theta_{i, 6} \cos \left(2 q_{i, 4}\right) \\
& +\frac{1}{2}\left(L_{4}\left(\theta_{i, 5}+\theta_{i, 7}\right)+L_{6} \theta_{i, 12}\left(L_{4}-L_{5}\right)-L_{5} \theta_{i, 7}\right)\left(\dot{q}_{i, 5} \sin \left(q_{i, 5}\right)\right. \\
& \left.-\left(2 \dot{q}_{i, 4}+\dot{q}_{i, 5}\right) \sin \left(2 q_{i, 4}+q_{i, 5}\right)\right)+\left(\dot{q}_{i, 4}+\dot{q}_{i, 5}\right) \sin \left(q_{i, 4}+q_{i, 5}\right) d_{20^{\prime}} \theta_{i, 10} \\
& -\left(\dot{q}_{i, 4}+\dot{q}_{i, 5}\right) \cos \left(2 q_{i, 5}+2 q_{i, 4}\right) L_{4} \theta_{i, 10}+d_{2 \_0^{\prime}} \dot{q}_{i, 4}\left(\theta_{i, 8}+\theta_{i, 6}\right) \sin \left(q_{i, 4}\right) \\
& C_{2,3}=\frac{1}{2} \dot{q}_{i, 2}\left(L_{6}^{2} \theta_{i, 12}-2 L_{4} \theta_{i, 5}\right) \sin \left(2 q_{i, 4}\right)-\dot{q}_{i, 2} \cos \left(2 q_{i, 5}+2 q_{i, 4}\right) L_{4} \theta_{i, 10} \\
& +\frac{1}{2} \dot{q}_{i, 2}\left(\left(L_{5}-L_{4}\right)^{2}\left(\theta_{i, 12}+\theta_{i, 11}\right)-2 L_{4} \theta_{i, 9}\right) \sin \left(2 q_{i, 5}+2 q_{i, 4}\right) \\
& +\left(\dot{q}_{i, 2} d_{20^{\prime}} \theta_{i, 10}+d_{6} \theta_{i, 11}\left(\dot{q}_{i, 4}+\dot{q}_{i, 5}\right)\left(L_{5}-L_{4}\right)\right) \sin \left(q_{i, 4}+q_{i, 5}\right)
\end{aligned}
$$




$$
\begin{aligned}
& -\left(\left(\left(L_{5}-L_{4}\right)\left(\theta_{i, 12}+\theta_{i, 11}\right)+\theta_{i, 9}\right) d_{2_{-} 0^{\prime}}\right) \dot{q}_{i, 2} \cos \left(q_{i, 4}+q_{i, 5}\right) \\
& +\dot{q}_{i, 2}\left(\left(L_{5}-L_{4}\right)\left(L_{6} \theta_{i, 12}+\theta_{i, 7}\right)-L_{4} \theta_{i, 5}\right) \sin \left(2 q_{i, 4}+q_{i, 5}\right) \\
& +\left(\dot{q}_{i, 4} d_{6} \theta_{i, 8}-\dot{q}_{i, 2}\left(L_{6} \theta_{i, 12}+\theta_{i, 7}+\theta_{i, 5}\right) d_{2 \_0^{\prime}}\right) \cos \left(q_{i, 4}\right) \\
& +\left(\dot{q}_{i, 2}\left(\theta_{i, 8}+\theta_{i, 6}\right) d_{2 \_0^{\prime}}+\dot{q}_{i, 4} d_{6} \theta_{i, 7}\right) \sin \left(q_{i, 4}\right) \\
& +\dot{q}_{i, 2}\left(\left(L_{5}-L_{4}\right) \theta_{i, 8}-L_{4} \theta_{i, 6}\right) \cos \left(2 q_{i, 4}+q_{i, 5}\right)-\dot{q}_{i, 2} L_{4} \theta_{i, 6} \cos \left(2 q_{i, 4}\right)
\end{aligned}
$$

$$
\begin{aligned}
C_{2,4}= & \left(\left(\dot{q}_{i, 4}+\dot{q}_{i, 5}\right)\left(L_{5}-L_{4}\right) d_{6} \theta_{i, 11}+\dot{q}_{i, 2} d_{2 \_} \theta_{i, 10}\right) \sin \left(q_{i, 4}+q_{i, 5}\right) \\
& +\frac{1}{2} \dot{q}_{i, 2}\left(\left(L_{5}-L_{4}\right)^{2}\left(\theta_{i, 12}+\theta_{i, 11}\right)-2 L_{4} \theta_{i, 9}\right) \sin \left(2 q_{i, 5}+2 q_{i, 4}\right) \\
& -\left(\left(L_{5}-L_{4}\right)\left(\theta_{i, 12}+\theta_{i, 11}\right)+\theta_{i, 9}\right) d_{2 \_0^{\prime}} \dot{q}_{i, 2} \cos \left(q_{i, 4}+q_{i, 5}\right) \\
& -\dot{q}_{i, 2} L_{4} \theta_{i, 10} \cos \left(2 q_{i, 5}+2 q_{i, 4}\right) \\
& -\frac{1}{2} \dot{q}_{i, 2}\left(L_{4} \theta_{i, 6}+\theta_{i, 8}\left(L_{4}-L_{5}\right)\right)\left(\cos \left(q_{i, 5}\right)+\cos \left(2 q_{i, 4}+q_{i, 5}\right)\right) \\
& +\frac{1}{2} \dot{q}_{i, 2}\left(\left(L_{5}-L_{4}\right)\left(L_{6} \theta_{i, 12}+\theta_{i, 7}\right)-L_{4} \theta_{i, 5}\right)\left(\sin \left(2 q_{i, 4}+q_{i, 5}\right)-\sin \left(q_{i, 5}\right)\right)
\end{aligned}
$$

$$
\begin{aligned}
C_{3,2}= & \dot{q}_{i, 2} L_{4} \theta_{i, 10} \cos \left(2 q_{i, 5}+2 q_{i, 4}\right)+\dot{q}_{i, 2} L_{4} \theta_{i, 6} \cos \left(2 q_{i, 4}\right) \\
& +\dot{q}_{i, 2} d_{2 \_0^{\prime}}\left(\theta_{i, 9}+\left(L_{5}-L_{4}\right)\left(\theta_{i, 12}+\theta_{i, 11}\right)\right) \cos \left(q_{i, 4}+q_{i, 5}\right) \\
& -\dot{q}_{i, 2} d_{2 \_0^{\prime}} \sin \left(q_{i, 4}+q_{i, 5}\right) \theta_{i, 10}+\frac{1}{2} \dot{q}_{i, 2}\left(2 L_{4} \theta_{i, 5}-L_{6}^{2} \theta_{i, 12}\right) \sin \left(2 q_{i, 4}\right) \\
& +\dot{q}_{i, 2}\left(L_{4} \theta_{i, 5}-\left(L_{5}-L_{4}\right)\left(L_{6} \theta_{i, 12}+\theta_{i, 7}\right)\right) \sin \left(2 q_{i, 4}+q_{i, 5}\right) \\
& +\frac{1}{2} \dot{q}_{i, 2}\left(-\left(L_{5}-L_{4}\right)^{2}\left(\theta_{i, 12}+\theta_{i, 11}\right)+2 L_{4} \theta_{i, 9}\right) \sin \left(2 q_{i, 5}+2 q_{i, 4}\right) \\
& +\dot{q}_{i, 2} d_{2 \_0^{\prime}}\left(\theta_{i, 5}+\theta_{i, 7}+L_{6} \theta_{i, 12}\right) \cos \left(q_{i, 4}\right)-\dot{q}_{i, 2} d_{2 \_0^{\prime}}\left(\theta_{i, 8}+\theta_{i, 6}\right) \sin \left(q_{i, 4}\right) \\
& +\dot{q}_{i, 2}\left(L_{4} \theta_{i, 6}+\theta_{i, 8}\left(L_{4}-L_{5}\right)\right) \cos \left(2 q_{i, 4}+q_{i, 5}\right)
\end{aligned}
$$

$$
\begin{aligned}
C_{3,3}= & \frac{1}{2} L_{4}\left(\dot{q}_{i, 4}+\dot{q}_{i, 5}\right)\left(\left(2 L_{5}-L_{4}\right)\left(\theta_{i, 12}+\theta_{i, 11}\right)+2 \theta_{i, 9}\right) \sin \left(2 q_{i, 5}+2 q_{i, 4}\right) \\
& -\frac{1}{2} \dot{q}_{i, 5}\left(\left(2 L_{5} L_{6}-L_{6} L_{4}+L_{5} L_{4}\right) \theta_{i, 12}+\left(2 L_{5}-L_{4}\right) \theta_{i, 7}+\theta_{i, 11} L_{5} L_{4}\right. \\
& \left.+L_{4}\left(\theta_{i, 9}-\theta_{i, 5}\right)\right) \sin \left(q_{i, 5}\right)+L_{4}\left(\dot{q}_{i, 4}+\dot{q}_{i, 5}\right) \theta_{i, 10} \cos \left(2 q_{i, 5}+2 q_{i, 4}\right) \\
& +\frac{1}{2} L_{4}\left(2 \dot{q}_{i, 4}+\dot{q}_{i, 5}\right)\left(\left(L_{5}+L_{6}\right) \theta_{i, 12}+\theta_{i, 7}+L_{5} \theta_{i, 11}+\theta_{i, 5}+\theta_{i, 9}\right) \\
& \times \sin \left(2 q_{i, 4}+q_{i, 5}\right)+L_{4} \dot{q}_{i, 4}\left(\theta_{i, 8}+\theta_{i, 6}\right) \cos \left(2 q_{i, 4}\right) \\
& +\frac{1}{2} L_{4} \dot{q}_{i, 4}\left(\left(L_{4}+2 L_{6}\right) \theta_{i, 12}+2 \theta_{i, 7}+L_{4} \theta_{i, 11}+2 \theta_{i, 5}\right) \sin \left(2 q_{i, 4}\right) \\
& +\frac{1}{2} L_{4}\left(2 \dot{q}_{i, 4}+\dot{q}_{i, 5}\right)\left(\theta_{i, 8}+\theta_{i, 6}+\theta_{i, 10}\right) \cos \left(2 q_{i, 4}+q_{i, 5}\right) \\
& -\frac{1}{2} \dot{q}_{i, 5}\left(L_{4}\left(\theta_{i, 6}+\theta_{i, 10}\right)+\theta_{i, 8}\left(L_{4}-2 L_{5}\right)\right) \cos \left(q_{i, 5}\right)
\end{aligned}
$$

$$
\begin{aligned}
C_{3,4}= & \left(\frac{1}{2}\left(\theta_{i, 8}+\theta_{i, 10}+\theta_{i, 6}\right) \cos \left(2 q_{i, 4}+q_{i, 5}\right)+\theta_{i, 10} \cos \left(2 q_{i, 5}+2 q_{i, 4}\right)\right) L_{4} \\
& \times\left(\dot{q}_{i, 4}+\dot{q}_{i, 5}\right)-\frac{1}{2}\left(\dot{q}_{i, 4}+\dot{q}_{i, 5}\right)\left(L_{5} L_{4} \theta_{i, 12}+\left(2 L_{5}-L_{4}\right)\left(\theta_{i, 7}+L_{6} \theta_{i, 12}\right)\right.
\end{aligned}
$$




$$
\begin{aligned}
& \left.+\theta_{i, 11} L_{5} L_{4}+L_{4}\left(\theta_{i, 9}-\theta_{i, 5}\right)\right) \sin \left(q_{i, 5}\right)+\frac{1}{2} L_{4}\left(\dot{q}_{i, 4}+\dot{q}_{i, 5}\right)\left(\left(L_{5}+L_{6}\right) \theta_{i, 12}\right. \\
& \left.+\theta_{i, 7}+L_{5} \theta_{i, 11}+\theta_{i, 5}+\theta_{i, 9}\right) \sin \left(2 q_{i, 4}+q_{i, 5}\right) \\
& +\frac{1}{2} L_{4}\left(\dot{q}_{i, 4}+\dot{q}_{i, 5}\right)\left(\left(2 L_{5}-L_{4}\right)\left(\theta_{i, 12}+\theta_{i, 11}\right)+2 \theta_{i, 9}\right) \sin \left(2 q_{i, 5}+2 q_{i, 4}\right) \\
+ & \frac{1}{2}\left(\dot{q}_{i, 4}+\dot{q}_{i, 5}\right)\left(\left(2 L_{5}-L_{4}\right) \theta_{i, 8}-L_{4}\left(\theta_{i, 10}+\theta_{i, 6}\right)\right) \cos \left(q_{i, 5}\right) \\
C_{4,2}= & \dot{q}_{i, 2} d_{2_{-} 0^{\prime}}\left(\left(L_{5}-L_{4}\right)\left(\theta_{i, 12}+\theta_{i, 11}\right)+\theta_{i, 9}\right) \cos \left(q_{i, 4}+q_{i, 5}\right) \\
+ & \frac{1}{2} \dot{q}_{i, 2}\left(\left(L_{5}-L_{4}\right)\left(L_{6} \theta_{i, 12}+\theta_{i, 7}\right)-L_{4} \theta_{i, 5}\right)\left(\sin \left(q_{i, 5}\right)-\sin \left(2 q_{i, 4}+q_{i, 5}\right)\right) \\
+ & \frac{1}{2} \dot{q}_{i, 2}\left(2 L_{4} \theta_{i, 9}-\left(\theta_{i, 12}+\theta_{i, 11}\right)\left(L_{5}-L_{4}\right)^{2}\right) \sin \left(2 q_{i, 5}+2 q_{i, 4}\right) \\
+ & \dot{q}_{i, 2} L_{4} \theta_{i, 10} \cos \left(2 q_{i, 5}+2 q_{i, 4}\right)-\dot{q}_{i, 2} d_{20^{\prime}} \theta_{i, 10} \sin \left(q_{i, 4}+q_{i, 5}\right) \\
+ & \frac{1}{2} \dot{q}_{i, 2}\left(L_{4} \theta_{i, 6}-\left(L_{5}-L_{4}\right) \theta_{i, 8}\right)\left(\cos \left(q_{i, 5}\right)+\cos \left(2 q_{i, 4}+q_{i, 5}\right)\right) \\
C_{4,3}= & \frac{1}{2} \dot{q}_{i, 4}\left(L_{4}\left(\theta_{i, 9}-\theta_{i, 5}+\theta_{i, 11} L_{5}\right)+\left(2 L_{5} L_{6}-L_{6} L_{4}+L_{5} L_{4}\right) \theta_{i, 12}\right. \\
& \left.+\left(2 L_{5}-L_{4}\right) \theta_{i, 7}\right) \sin \left(q_{i, 5}\right)+L_{4}\left(\dot{q}_{i, 4}+\dot{q}_{i, 5}\right) \theta_{i, 10} \cos \left(2 q_{i, 5}+2 q_{i, 4}\right) \\
& +\frac{1}{2} \dot{q}_{i, 4} L_{4}\left(\left(L_{5}+L_{6}\right) \theta_{i, 12}+\theta_{i, 7}+L_{5} \theta_{i, 11}+\theta_{i, 5}+\theta_{i, 9}\right) \sin \left(2 q_{i, 4}+q_{i, 5}\right) \\
& +\frac{1}{2} \dot{q}_{i, 4} L_{4}\left(\theta_{i, 8}+\theta_{i, 6}+\theta_{i, 10}\right) \cos \left(2 q_{i, 4}+q_{i, 5}\right) \\
& +\frac{1}{2} L_{4}\left(\dot{q}_{i, 4}+\dot{q}_{i, 5}\right)\left(\left(2 L_{5}-L_{4}\right)\left(\theta_{i, 12}+\theta_{i, 11}\right)+2 \theta_{i, 9}\right) \sin \left(2 q_{i, 5}+2 q_{i, 4}\right) \\
& +\frac{1}{2} \dot{q}_{i, 4}\left(L_{4}\left(\theta_{i, 6}+\theta_{i, 10}\right)+\theta_{i, 8}\left(L_{4}-2 L_{5}\right)\right) \cos \left(q_{i, 5}\right) \\
= & \frac{1}{2} L_{4}\left(\dot{q}_{i, 4}+\dot{q}_{i, 5}\right)\left(\left(2 L_{5}-L_{4}\right)\left(\theta_{i, 12}+\theta_{i, 11}\right)+2 \theta_{i, 9}\right) \sin \left(2 q_{i, 5}+2 q_{i, 4}\right) \\
& +L_{4}\left(\dot{q}_{i, 4}+\dot{q}_{i, 5}\right) \theta_{i, 10} \cos \left(2 q_{i, 5}+2 q_{i, 4}\right) \\
\left.C_{4,4}\right) & \\
& \\
&
\end{aligned}
$$

\section{Entries of the gravity vector $g\left(q_{i}\right)$}

The entries of the gravity vector $g\left(q_{i}\right) \in \mathbb{R}^{4}$ as function of the generalized coordinates $q_{i}=\left[\begin{array}{llll}q_{i, 1} & q_{i, 2} & q_{i, 4} & q_{i, 5}\end{array}\right]^{T}$ of robot $i, i=1,2$, the parameters $\theta_{i, j}, j=1, \ldots 32$, and the acceleration due to gravity $g=9.81 \mathrm{~m} / \mathrm{s}^{2}$, are given by

$$
\begin{gathered}
g_{1}=g_{2}=0 \\
g_{3}=-g\left(\theta_{i, 9}+\theta_{i, 12} L_{5}+L_{5} \theta_{i, 11}\right) \sin \left(q_{i, 4}+q_{i, 5}\right)-g\left(\theta_{i, 6}+\theta_{i, 8}\right) \cos \left(q_{i, 4}\right) \\
-g\left(\theta_{i, 5}+\theta_{i, 12}\left(L_{6}+L_{4}\right)+L_{4} \theta_{i, 11}+\theta_{i, 7}\right) \sin \left(q_{i, 4}\right)-g \theta_{i, 10} \cos \left(q_{i, 4}+q_{i, 5}\right) \\
g_{4}=-g\left(\theta_{i, 9}+\theta_{i, 12} L_{5}+L_{5} \theta_{i, 11}\right) \sin \left(q_{i, 4}+q_{i, 5}\right)-g \theta_{i, 10} \cos \left(q_{i, 4}+q_{i, 5}\right)
\end{gathered}
$$




\section{Entries of the vector of friction forces $f\left(\dot{q}_{i}\right)$}

The friction forces $f\left(\dot{q}_{i}\right) \in \mathbb{R}^{4}$ in the transposer robot are model by (6.2), such that the entries of $f\left(\dot{q}_{i}\right)$ can be written as function of the generalized velocities $\dot{q}_{i}=\left[\begin{array}{llll}\dot{q}_{i, 1} & \dot{q}_{i, 2} & \dot{q}_{i, 4} & \dot{q}_{i, 5}\end{array}\right]^{T}$ for the $i$ robot, and the parameters $\theta_{i}, j, i=1,2$, $j=1, \ldots 32$,

$$
\begin{aligned}
& f_{1}\left(\dot{q}_{i, 1}\right)=\theta_{i, 13} \dot{q}_{i, 1}+\theta_{i, 17}\left(1-\frac{2}{1+e^{2 \theta_{i, 25} \dot{q}_{i, 1}}}\right)+\theta_{i, 21}\left(1-\frac{2}{1+e^{2 \theta_{i, 29} \dot{q}_{i, 1}}}\right) \\
& f_{2}\left(\dot{q}_{i, 2}\right)=\theta_{i, 14} \dot{q}_{i, 2}+\theta_{i, 18}\left(1-\frac{2}{1+e^{2 \theta_{i, 26} \dot{q}_{i, 2}}}\right)+\theta_{i, 22}\left(1-\frac{2}{1+e^{2 \theta_{i, 30} \dot{q}_{i, 2}}}\right) \\
& f_{3}\left(\dot{q}_{i, 4}\right)=\theta_{i, 15} \dot{q}_{i, 4}+\theta_{i, 19}\left(1-\frac{2}{1+e^{2 \theta_{i, 27} \dot{q}_{i, 4}}}\right)+\theta_{i, 23}\left(1-\frac{2}{1+e^{2 \theta_{i, 31} \dot{q}_{i, 4}}}\right) \\
& f_{4}\left(\dot{q}_{i, 5}\right)=\theta_{i, 16} \dot{q}_{i, 5}+\theta_{i, 20}\left(1-\frac{2}{1+e^{2 \theta_{i, 28} \dot{q}_{i, 5}}}\right)+\theta_{i, 24}\left(1-\frac{2}{1+e^{2 \theta_{i, 32} \dot{q}_{i, 5}}}\right)
\end{aligned}
$$

\section{Estimated parameters}

Physically in the Dynamics and Control Laboratory the robots for which the parameters have been estimated R1 and R2 correspond to the robot with plate numbers 677528 and 669358 respectively

The physical parameters $\theta_{i, j}, i=1,2, j=1, \ldots 32$ of the transposer robots have been estimated by using an extended Kalman filter and the least square method, in a similar way to the work presented in (Kostic et al. 2001). The estimated parameters are listed in Table H.3, see (Rodriguez-Angeles et al. 2002a) for a physical interpretation of the parameters $\theta_{i}, j, i=1,2, j=1, \ldots 32$. The control $\tau_{i}$ for collecting the data to run the Kalman filter and the least square algorithm was set as a P-controller, with a harmonic signal as desired trajectory $q_{d}(t)$ with fundamental frequency of $0.4 \mathrm{~Hz}$.

As a manner of validation of the dynamic model (H.8) and (H.9) and the estimated physical parameters listed in Table H.3, a comparison study between measured $\tau_{i}$ and estimated $\tau_{e, i}$ input torques is carried out. Figures H.3 - H.6 show the estimated $\tau_{e i, j}$ (solid) and measured input control $\tau_{i, j}$ (dashed) for robots $\mathrm{R} 1$ and $\mathrm{R} 2, i=1,2$, and the joints $j=1,2,4,5$. The estimated input control $\tau_{e, i}$ is obtained from the dynamic model (6.1) and (6.2) and the estimated parameters listed in Table H.3, by using the measured variables $q_{i, j}, \dot{q}_{i, j}$ and $\ddot{q}_{i, j}$ originated by the measured torque $\tau_{i, j}$.

It is worth to mention that the same methodology, was used in both robots, i.e. controller and trajectories. However, Figures H.3 - H.6 show better fitting between the estimated and measured input control for robot $\mathrm{R} 2$ than for robot $\mathrm{R} 1$. Thus the dynamic model (H.8) and (H.9) and the estimated parameters, listed in Table H.3, are a better representation of the dynamics of robot $\mathrm{R} 2$ than robot $\mathrm{R} 1$. The differences in the estimation of the parameters may be due to weariness in the servomotors and transmission elements, or to a better maintenance of robot R2. Indeed by manual inspection of the robots it is apparent that the robot $\mathrm{R} 1$ presents more weariness in the servomotors and transmission elements than robot R2. 


\begin{tabular}{|c|c|c|}
\hline Parameter & Robot R1 $(i=1)$ & Robot R2 $(i=2)$ \\
\hline$\theta_{i, 1}$ & 147.0161 & 121.3049 \\
\hline$\overline{\theta_{i, 2}}$ & 2.1448 & 0.3107 \\
\hline$\theta_{i, 3}$ & -0.6363 & 4.1955 \\
\hline$\theta_{i, 4}$ & 0.5931 & 1.7453 \\
\hline$\theta_{i, 5}$ & 0.1701 & 0.8316 \\
\hline$\theta_{i, 6}$ & -0.0561 & 0.8687 \\
\hline$\theta_{i, 7}$ & 0.8392 & 0.8105 \\
\hline$\theta_{i, 8}$ & 1.9397 & 1.6721 \\
\hline$\theta_{i}, 9$ & -0.1428 & -0.1879 \\
\hline$\theta_{i, 10}$ & 1.7807 & 1.7850 \\
\hline$\theta_{i, 11}$ & 0.1498 & 0.8759 \\
\hline$\theta_{i, 12}$ & 4.4844 & 4.1328 \\
\hline$\theta_{i, 13}$ & 83.2945 & 97.2600 \\
\hline$\theta_{i, 14}$ & 11.2104 & 9.0999 \\
\hline$\theta_{i, 15}$ & 16.6527 & 11.6257 \\
\hline$\theta_{i, 16}$ & 13.6684 & 9.6229 \\
\hline$\theta_{i, 17}$ & -72.6918 & -54.9912 \\
\hline$\theta_{i, 18}$ & -32.9333 & 18.4710 \\
\hline$\theta_{i, 19}$ & 5.2337 & -3.5232 \\
\hline$\theta_{i, 20}$ & -3.0435 & -5.8564 \\
\hline$\theta_{i, 21}$ & -85.4138 & -46.5915 \\
\hline$\theta_{i, 22}$ & -42.4819 & 11.1605 \\
\hline$\theta_{i, 23}$ & -4.3254 & 2.2684 \\
\hline$\theta_{i, 24}$ & 5.5640 & 8.2304 \\
\hline$\overline{\theta_{i, 25}}$ & 149.9624 & 150.3190 \\
\hline$\theta_{i, 26}$ & 142.7894 & 136.8945 \\
\hline$\theta_{i, 27}$ & 8.6392 & -35.3699 \\
\hline$\theta_{i, 28}$ & 27.6979 & 36.0641 \\
\hline$\theta_{i, 29}$ & -100.2648 & -98.9881 \\
\hline$\theta_{i}, 30$ & -142.2786 & -170.4702 \\
\hline$\theta_{i, 31}$ & -1.8278 & -89.3236 \\
\hline$\theta_{i}, 32$ & 12.0224 & 16.2942 \\
\hline
\end{tabular}

Table H.3: Estimated parameters for the CFT transposer robots. 

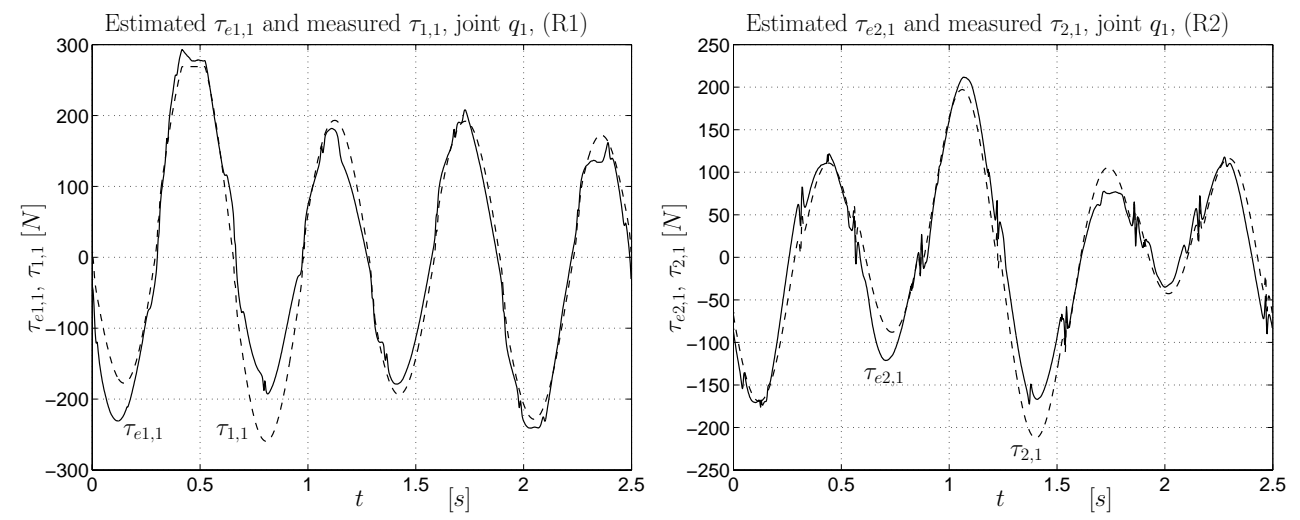

Figure H.3: Estimated $\tau_{e i, 1}$ (solid) and measured torque $\tau_{i, 1}$ (dashed), joint $q_{1}$, robots $\mathrm{R} 1$ and R2.
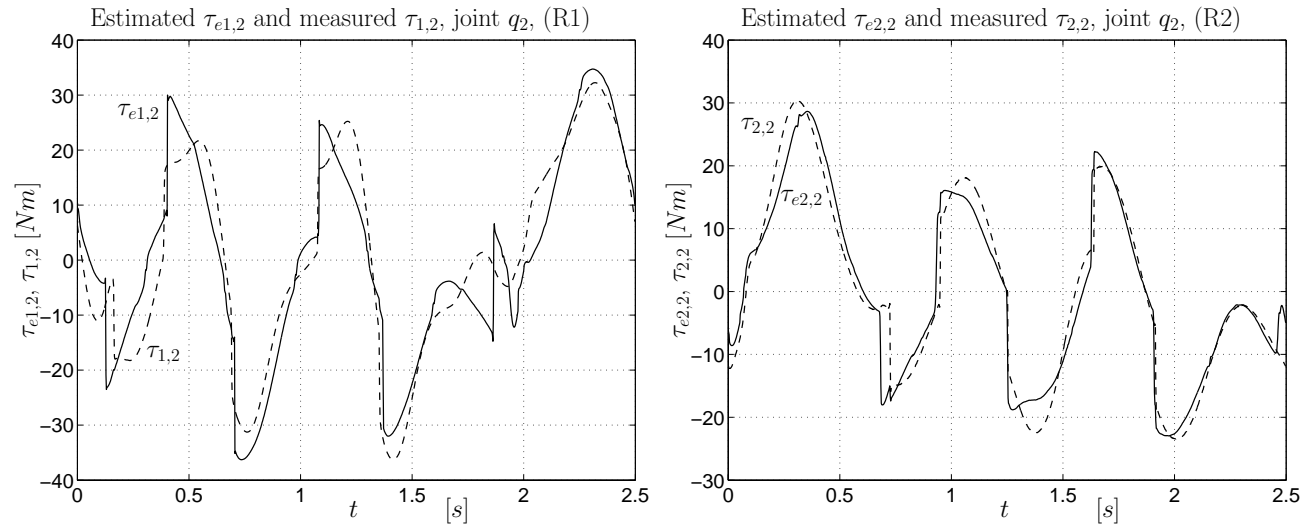

Figure H.4: Estimated $\tau_{e i, 2}$ (solid) and measured torque $\tau_{i, 2}$ (dashed), joint $q_{2}$, robots $\mathrm{R} 1$ and $\mathrm{R} 2$. 

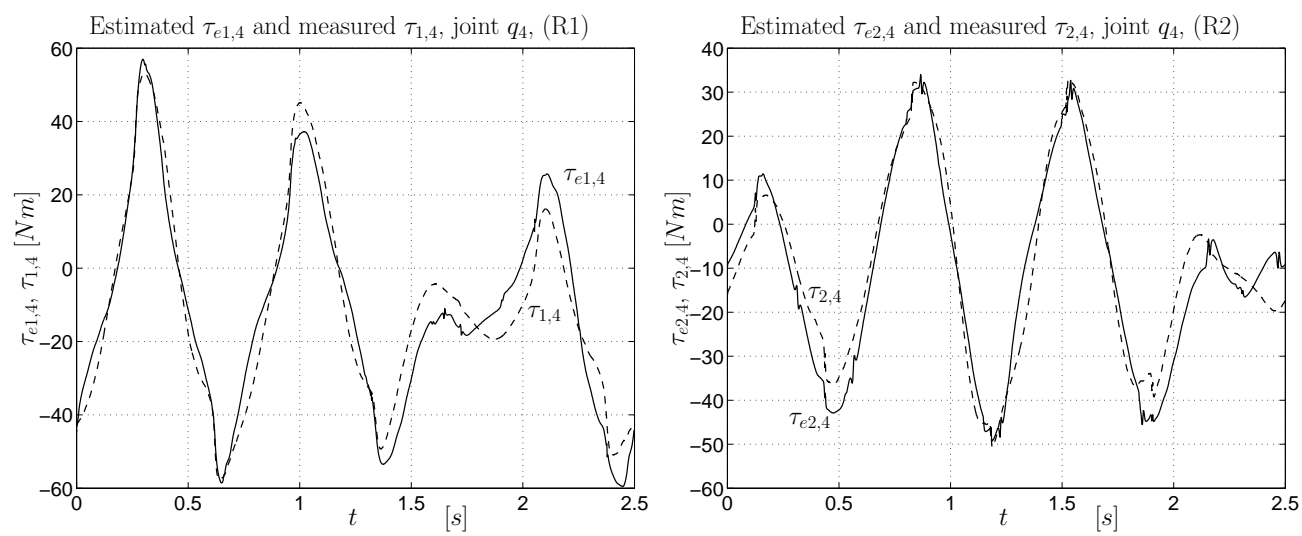

Figure H.5: Estimated $\tau_{e i, 4}$ (solid) and measured torque $\tau_{i, 4}$ (dashed), joint $q_{4}$, robots $\mathrm{R} 1$ and $\mathrm{R} 2$.
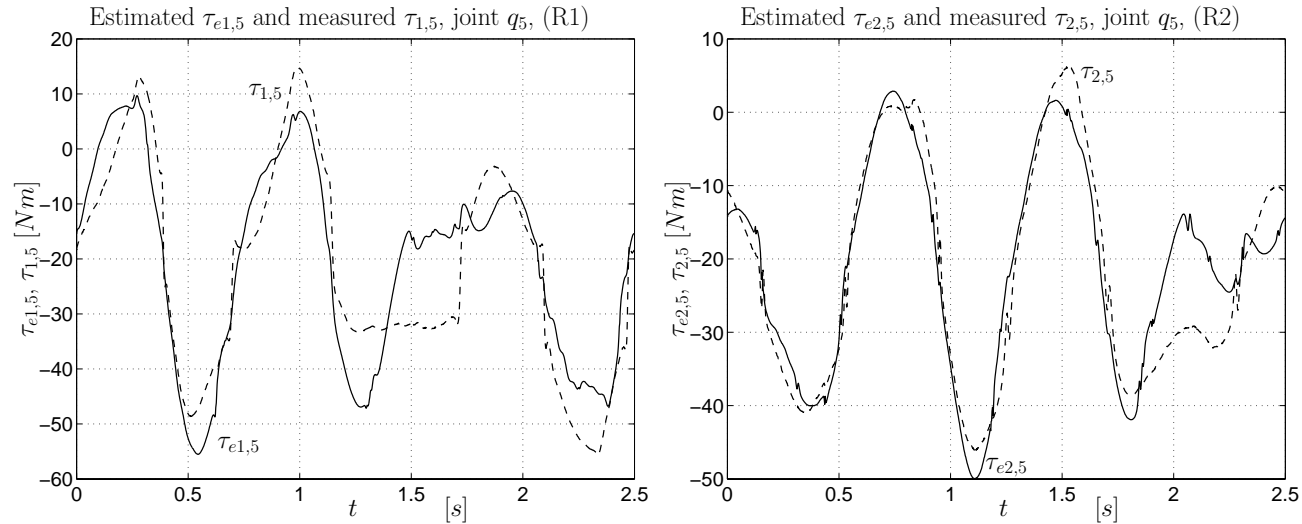

Figure H.6: Estimated $\tau_{e i, 5}$ (solid) and measured torque $\tau_{i, 5}$ (dashed), joint $q_{5}$, robots $\mathrm{R} 1$ and $\mathrm{R} 2$. 


\section{Bibliography}

Armstrong-Hélouvry, B. (1991). Control of Machines with Friction. Kluwer Academic Publishers. Boston.

Armstrong-Hélouvry, B. (1993). Stick-slip and control in low-speed motion. IEEE Transactions on Automatic Control 38, 1483-1496.

Armstrong-Hélouvry, B., P. Dupont and C. Canudas de Wit (1994). A survey of models, analysis tools and compensation methods for the control of machines with friction. Automatica 30, 1083-1138.

Berghuis, H. (1993). Model-based Robot Control: from Theory to Practice. PhD thesis. University of Twente, The Netherlands.

Berghuis, H. and H. Nijmeijer (1994). Robust control of robots via linear estimated state feedback. IEEE Transactions on Automatic Control 39, 2159-2162.

Blekhman, I. I. (1988). Synchronization in Science and Technology. ASME Press Translations. New York.

Blekhman, I. I., P. S. Landa and M. G. Rosenblum (1995). Synchronization and chaotization in interacting dynamical systems. ASME Applied Mechanical Review 48, 733-752.

Blekhman, I. I., A. L. Fradkov, H. Nijmeijer and A. Yu Pogromsky (1997). On self-synchronization and controlled synchronization. Systems and Control Letters 31, 299-305.

Bliman, P. A. and M. Sorine (1995). Easy-to-use realistic dry friction models for automatic control. In: Proceedings of the 3rd. European Control Conference. pp. $3788-3794$.

Book, W. J. (1984). Recursive lagrangian dynamics of flexible manipulator arms. International Journal of Robotics Research 3, 87-101.

Breedveld, P. C., R. C. Rosenberg and T. Zhou (1991). Bibliography of bond graph theory and application. Journal of the Franklin Institute 328, 1067-1109.

Brockett, R.W. (1983). Asymptotic stability and feedback stabilization. In: Differential Geometric Control Theory, Brockett,R.W., Millman, R.S., and Sussman, H.J., Eds.. pp. 181-191. Birkhäuser. Boston, MA, USA. 
Brunt, M. (1998). Coordination of Redundant Systems. PhD thesis. Technical University Delft, The Netherlands.

Chen, Y. H. and G. Leitmann (1987). Robustness of uncertain systems in the absence of matching assumptions. International Journal of Control 45, 1527-1542.

Chovotov, V.A. (1991). Spacecraft attitude dynamics and control. Krieger Publishing Company. Malabar, FL.

Canudas de Wit, C. and O.J. Sordalen (1992). Exponential stabilization of mobile robots with nonholonomic constraints. IEEE Transactions on Automatic Control 37, 1791-1797.

Canudas de Wit, C., H. Olsson, K. J. Åström and P. Lischinsky (1995). A new model for control of systems with friction. IEEE Transactions on Automatic Control 40, 419-425.

Canudas de Wit, C. and P. Lischinsky (1997). Adaptive friction compensation with partially known dynamic friction model. International Journal of Adaptive Control and Signal Processing 11, 65-80.

Craig, J. J. (1988). Adaptive Control of Mechanical Manipulators. Addison-Wesley. New York.

Cuomo, K. M., A. V. Oppenheim and S. H. Strogatz (1993). Synchronization of Lorenz-based chaotic circuits with application to communications. IEEE Transactions on Circuit Systems II: Analog and Digital Signal Processing 40, 626-633.

Desoer, C. A. and M. Vidyasagar (1975). Feedback Systems: Input-Output Properties. Academic Press. New York.

Dixon, W. E., E. Zergeroglu, D. M. Dawson and M. W. Hannan (2000). Global adaptive partial state feedback tracking control of rigid-link flexible-joint robots. Robotica 18, 325-336.

Dubey, R. V., T. F. Chan and S. E. Everett (1997). Variable damping impedance control of a bilateral telerobotics system. IEEE Control and Systems 17, 37-45.

Dupont, P. E. and E. P. Dunlap (1995). Friction modeling and PD compensation at very low velocities. ASME Journal of Dynamic Systems, Measurements and Control 117, 9-14.

Feng, L., Y. Koren and J. Borenstein (1993). Cross-coupling motion controller for mobile robots. IEEE Control Systems Magazine 13, 35-43.

Goldstein, H. (1980). Classical Mechanics. Addison-Wesley. Reading, MA.

Good, M. C., L. M. Sweet and K. L. Strobel (1985). Dynamic models for control systems design of integrated robot and drive systems. ASME Journal of Dynamic Systems, Measurement, and Control 107, 53-59.

Gray, C. M. (1994). Synchronous oscillations in neuronal systems: mechanisms and functions. Journal of Computational Neuroscience 1, 11-38. 
Guthart, G. S. and J. K. Salisbury Jr. (2000). The intuitive ${ }^{T M}$ telesurgery system: overview and application. In: Proceedings of the IEEE International Conference on Robotics and Automation. pp. 618-621.

Haessig, D. A. and B. Friedland (1991). On the modeling and simulation of friction. ASME Journal of Dynamic Systems, Measurements and Control 113, 354-362.

Han, J. D., Y. C. Wang, D. L. Tan and W. L. Xu (2000). Acceleration feedback control for direct-drive motor system. In: Proceedings of the 2000 IEEE/RSJ International Conference on Intelligent Robots and Systems. pp. 1068-1074.

Hensen, R. H. A., G. Z. Angelis, M. J. G. v. d. Molengraft, A. G. de Jager and J. J. Kok (2000). Grey-box modeling of friction: An experimental case-study. European Journal of Control 6, 258-267.

Hills, J. W. and J. F. Jensen (1998). Telepresence technology in medicine: principles and applications. In: Proceedings of the IEEE. Vol. 86. pp. 569-580.

Hirata, Y. and K. Kosuge (2000). Coordinated motion control of multiple robots without position information of each robot. In: Proceedings of the IEEE Conference on Desicion and Control. pp. 346-351.

Huijberts, H. J. C., H. Nijmeijer and R. M. A. Willems (2000). Regulation and controlled synchronization for complex dynamical systems. International Journal of Robust and Nonlinear Control 10, 336-377.

Huygens, C. (1673). Horoloqium Oscilatorium. Paris, France.

Jankovic, M. (1995). Observer based control for elastic joint robots. IEEE Transactions on Robotics and Automation 11, 618-623.

Johnson, R. C. (1990). Matrix Theory and Application. American Mathematical Society. Rhode Island.

Kadhim, A. H., T. K. Babu and D. O'Kelly (1992). Measurement of steady-state and transient load-angle, angular velocity, and acceleration using an optical encoder. IEEE Transactions on Instrumentation and Measurement 41, 486-489.

Kang, W. and H. Yeh (2002). Co-ordinated attitude control of multi-satellite systems. International Journal of Robust and Nonlinear Control 12, 185-205.

Khalil, H. K. (1996). Nonlinear Systems. Prentice Hall. Upper Saddle River, New Jersey.

Kocarev, L., K. S. Halle, K. Eckert and L. O. Chua (1992). Experimental demonstration of secure communication via chaotic synchronization. International Journal of Bifurcations and Chaos 2, 709-713.

Kostic, D., R. Hensen, B. de Jager and M. Steinbuch (2001). Modeling and identification of an RRR-robot. In: Proceedings of the IEEE Conference on Decision and Control. pp. 1144-1149.

Laopoulos, T. and C. Papageorgiou (1996). Microcontroller-based measurement of angular position, velocity and acceleration. In: Proceedings of the IEEE Instrumentation and Measurement Technology Conference. pp. 73-77. 
Lee, C. S. G. (1982). Robot arm kinematics, dynamics and control. IEEE Computer 15, 62-80.

Lee, H. K. and M. J. Chung (1998). Adaptive controller of a master-slave system for transparent teleoperation. Journal of Robotic Systems 15, 465-475.

Lefeber, E., J. Jakubiak, K. Tchoń and H. Nijmeijer (2001). Observer based kinematic tracking controllers for a unicycle-type mobile robot. In: Proceedings of the 2001 IEEE International Conference on Robotics and Automation. pp. 2084-2089.

Lewis, F. T., C. T. Abdallah and D. M. Dawson (1993). Control of Robot Manipulators. MacMillan. New York.

Lim, S. Y., D. M. Dawson, J. Hu and M. S. de Queiroz (1997). An adaptive link position tracking controller for rigid-link flexible-joint robots without velocity measurements. IEEE Transactions on Systems, Man, and Cybernetics 27, 412427.

Liu, Y. H., S. Arimoto, V. Parra-Vega and K. Kitagaki (1997). Decentralized adaptive control of multiple manipulators in cooperations. International Journal of Control 67, 649-673.

Liu, Y. H., Y. Xu and M. Bergerman (1999). Cooperation control of multiple manipulators with passive joints. IEEE Transactions on Robotics and Automation 15, 258-267.

Lozano, R. and B. Brogliato (1992). Adaptive control of robot manipulators with flexible joints. IEEE Transactions on Automatic Control 37, 174-181.

De Luca, A. and L. Lanari (1995). Robots with elastic joints are linearizable via dynamic feedback. In: Proceedings of the 34th IEEE Conference on Decision and Control. pp. 3895-3897.

De Luca, A. and P. Tomei (1996). Elastic Joints, In Theory of Robot Control, C. Canudas de Wit, B. Siciliano, and G. Bastin (Eds.). pp. 179-217. SpringerVerlag. New York.

De Luca, A. and P. Lucibello (1998). A general algorithm for dynamic feedback linearization of robots with elastic joints. In: Proceedings of the IEEE International Conference on Robotics and Automation. pp. 504-510.

De Luca, A. (1998). Decoupling and feedback linearization of robots with mixed rigid/elastic joints. International Journal of Robust and Nonlinear Control 8, 965-977.

Lygouras, J. N., K. A. Lalakos and P. G. Tsalides (1998). High-performance position detection and velocity adaptive measurement for closed-loop position control. IEEE Transactions on Instrumentation and Measurement 47, 978-985.

Mirollo, R. E. and S. H. Strogatz (1990). Synchronization of pulse-coupled biological oscillators. SIAM Journal on Applied Mathematics 50, 1645-1662.

Nicosia, S., P. Tomei and A. Tornambé (1988). A nonlinear observer for elastic robots. IEEE Journal on Robotics and Automation 4, 45-52. 
Nicosia, S. and P. Tomei (1995). A tracking controller for flexible joint robots using only link feedback. IEEE Transactions on Automatic Control 40, 885-890.

Olsson, H., K. J. Åström, C. Canudas de Wit, M. Gäfvert and P. Lischinsky (1998). Friction models and friction compensation. European Journal of Control 4, 176195 .

Olsson, H. and K. J. Åström (2001). Friction generated limit cycles. IEEE Transactions on Control Systems Technology 9, 629-636.

Otten, G., T. J. A. de Vries, J. van Amerongen and A. M. Rankers (1997). Linear motor motion control using a learning feedforward controller. IEEE/ASME Transactions on Mechatronics 2, 179-186.

Ovaska, S. J. and S. Vliviita (1998). Angular acceleration measurement: a review. IEEE Transactions on Instrumentation and Measurement 47, 1211-1217.

Paden, B. and R. Panja (1988). Globally asymptotically stable 'PD+' controller for robot manipulators. International Journal of Control 47, 1697-1712.

Pecora, L. M. and T. L. Carroll (1990). Synchronization in chaotic systems. Physics Review Letters 64, 821-824.

$\mathrm{Qu}, \mathrm{Z}$. (1995). Input-output robust tracking control design for flexible joint robots. IEEE Transactions on Automatic Control 40, 78-83.

Rayleigh, J. (1945). Theory of Sound. Dover. New York.

Rodriguez-Angeles, A. and H. Nijmeijer (2001a). Co-ordination of two robot manipulators via nonlinear estimated state feedback. In: Proceedings of the 5th IFAC Symposium "Nonlinear Control Systems" NOLCOS'01. pp. 255-260.

Rodriguez-Angeles, A. and H. Nijmeijer (2001b). Coordination of two robot manipulators based on position measurements only. International Journal of Control 74, 1311-1323.

Rodriguez-Angeles, A. and H. Nijmeijer (2002a). Mutual synchronizing of robots via estimated state feedback: a cooperative approach. To be submitted.

Rodriguez-Angeles, A. and H. Nijmeijer (2002b). Synchronizing tracking control for flexible joint robots via estimated state feedback. Submitted to ASME Journal of Dynamic Systems, Measurements, and Control.

Rodriguez-Angeles, A., D. Lizarraga, H. Nijmeijer and H. A. van Essen (2002a). Modelling and identification of the CFT-transposer robot. Technical Report 2002.52. Eindhoven University of Technology, Dynamics and Control Technology Group. Eindhoven, The Netherlands.

Rodriguez-Angeles, A., H. Nijmeijer and H. A. van Essen (2002b). Coordination of robot manipulators. In: Proceedings of the 8th Mechatronics Forum International Conference, Mechatronics 2002. pp. 1484-1493. 
Rodriguez-Angeles, A., H. Nijmeijer and H. A. van Essen (2002c). Coordination of rigid and flexible joint robot manipulators, In Advanced Dynamics and Control of Structures and Machines, H. Irschik and K. Schlander (Eds.). pp. -. SpringerVerlag. New York.

Samson, C. and K. Ait-Abderrahim (1991). Feedback control of a nonholonomic wheeled cart in cartesian space. In: Proceedings of the IEEE International Conference on Robotics and Automation. pp. 1136-1141.

Sastry, S. (1999). Nonlinear systems analysis, stability and control. Springer-Verlag. New York.

Siméon, T., S. Leroy and J.-P. Laumond (2002). Path coordination for multiple mobile robots: a resolution-complete algorithm. IEEE Transactions on Robotics and Automation 18, 42-49.

Spong, M. W. (1987). Modelling and control of elastic joint robots. ASME Journal of Dynamic Systems, Measurement, and Control 109, 310-319.

Spong, M. W. and M. Vidyasagar (1989). Robot Dynamics and Control. Wiley. New York.

Stewart, G.W. and J.-G. Sun (1990). Matrix Perturbation Theory. Academic Press. London.

Stramigioli, S. (1998). From Differentiable Manifolds to Interactive Robot Control. PhD thesis. Technical University Delft, The Netherlands.

Sun, D. and J.K. Mills (2002). Adaptive synchronized control for coordination of two robot manipulators. In: Proceedings of the 2002 IEEE International Conference on Robotics and Automation. pp. 976-981.

Sun, D., H.N. Dong and S.K. Tso (2002). Tracking stabilization of differential mobile robots using adaptive synchronized control. In: Proceedings of the 2002 IEEE International Conference on Robotics and Automation. pp. 2638-2643.

Sun, H. and G. T.-C. Chiu (2002). Motion synchronization for dual-cylinder electrohydraulic lift systems. IEEE/ASME Transactions on Mechatronics 7, 171-181.

Tomei, P. (1991). A simple PD controller for robots with elastic joints. IEEE Transactions on Automatic Control 36, 1208-1213.

Torre, V. (1976). A theory on synchronization of heart pace-maker cells. Journal of Theoretical Biology 61, 55-71.

Wang, P.K.C., A. Sparks and S. Banda (1996). Co-ordination and control of multiple microspacecraft moving in formation. The Journal of the Astronautical Sciences 44, 315-355.

Winfree, A. T. (1980). The Geometry of Biological Time. Springer. New York.

Yamaguchi, H., T. Arai and G. Beni (2001). A distributed control scheme for multiple robotic vehicles to make group formations. Robotics and Autonomous Systems 36, 125-147. 


\section{Summary}

This thesis addresses the possibility of inducing synchronous behavior in mechanical systems. Synchronization of mechanical systems is important since it gives rise to robust and dexterous systems. Synchronization forces a coordination or interaction between separate parts of a composed system, so that more complex and demanding tasks can be executed, mainly tasks that cannot be executed by an individual part of the composed system. The synchronization behavior can induce an optimal set of interactions between systems, such that minimization of some functional can be achieved, e.g. task time execution, norm of the relative errors between the systems, payload distribution. The performance of the systems is in this way improved. Examples of synchronization in mechanical systems are tele-operated master-slave robots used in surgery and hazardous environments, vibro-machinery used in mining, multirobot systems for production systems or in special applications such as multi-fingered robot hands.

The goal of the thesis can be stated as to design controller schemes that ensure synchronization of two or more mechanical systems, either identical or different. The major assumption, which restricts the design of the controllers, is that only position measurements in all the systems are available. The absence of measurements of velocities and accelerations is solved by considering model based observers. The measured positions and the estimated velocities and accelerations, obtained by the observers, are used in feedback controllers, which ensure synchronization of the mechanical systems.

In the thesis special attention is given to robot manipulators for which mutual (cooperative) and external (coordination) synchronization is considered. Nevertheless, the developed ideas can be extended to more general systems, such as mobile robot systems, multi-satellite systems, communication systems, and electro-mechanical systems such as electrical generators. In particular the proposed synchronization ideas are extended to formation (platooning) of mobile robots and attitude formation of satellites, for which general ideas and guidelines are presented. The proposed extensions are validated by simulations.

The results in the thesis are supported by analytical proofs and experimental results. The experiments show the predicted synchronization behavior and thus validate the practical viability of the proposed synchronization schemes. Two industrial transposer robots form the experimental setup. The robots are installed at the Dynamics and

Control Technology laboratory of the Department of Mechanical Engineering at the Eindhoven University of Technology. 
In the particular case of synchronization of robot manipulators several problems have to be faced at the practical implementation stage. The structure and design of the robots result in dynamical and structural problems such as friction phenomena, joint flexibility, high order and complex dynamic models, etc.. Many of these problems are addressed in the thesis. The way these practical problems are solved in order to implement the proposed synchronization schemes is discussed throughout the thesis.

Further extensions of the proposed synchronization ideas and recommendations for research in controlled synchronization of systems are pointed out. Most of the recommended lines of research represent challenging open problems that have arisen from this thesis. 


\section{Samenvatting}

Dit proefschrift onderzoekt de mogelijkheden voor het synchroniseren van mechanische systemen. Synchronisatie, van met name mechanische systemen, is belangrijk omdat het kan leiden tot meer robuuste en flexibele systemen. Synchronisatie betekent coördinatie of interactie tussen de samenstellende delen van systeem zodat complexere of ingewikkeldere taken kunnen worden uitgevoerd, met name taken die niet door een enkel systeem kunnen worden uitgevoerd. Synchronisatie leidt in zo'n geval tot (optimale) interactie tussen individuele systemen zodat de performance van het totale systeem verbeterd wordt, bijvoorbeeld kleinere productie tijd, een minimale relatieve fout tussen de individuele systemen of een optimale last distributie. Voorbeelden van synchronisatie in mechanische systemen zijn op afstand bediende masterslave robots voor operaties in ziekenhuizen of inspecties in schadelijke omgevingen, trillingscompensatoren in mijnen, multi-robot systemen in productie systemen of speciale toepassingen zoals meervingerige kunsthanden.

Het doel van dit proefschrift is het ontwerpen van regelaars die synchronisatie tussen twee of meer mechanische systemen, zowel identieke als verschillende systemen, garanderen. De voornaamste beperking die we aan het ontwerp stellen, is dat alleen positie informatie van alle samenstellende systemen via metingen beschikbaar is voor de regelaar. De ontbrekende informatie over snelheden en versnellingen wordt werkregen middels model-gebaseerde waarnemers. De gemeten posities en de geschatte snelheden en versnellingen worden gebruikt in feedback regelaars, die de synchronisatie van de mechanische systemen garanderen.

De aandacht in dit proefschrift is met name gericht op robot manipulatoren. Zowel samenwerkende (of gemeenschappelijke) synchronisatie als coördinerende (of externe) synchronisatie schema's worden onderzocht. De resultaten kunnen echter gegeneraliseerd worden naar meer algemene systemen, zoals mobiele robots, multi-satelliet systemen, communicatie processen en elektromechanische systemen zoals elektrische generatoren. In dit proefschrift worden de voorgestelde synchronisatie schema's toegepast op formatie-problemen van mobiele robots en satellieten. De voorgestelde uitbreidingen worden gevalideerd middels simulaties. Hierbij worden enkele algemene ideeën en richtlijnen gepresenteerd.

De resultaten van dit proefschrift worden onderbouwd door analytische bewijzen en experimentele resultaten. De experimenten tonen de voorspelde synchronisatie en ondersteunen de praktische haalbaarheid van de voorgestelde synchronisatie schema's. De experimentele opstelling bestaat uit twee industriële transposer robots in het Dy- 
namics and Control laboratorium van de faculteit Werktuigbouwkunde van de Technische Universiteit Eindhoven.

Bij de praktische implementatie van synchronisatie van robot manipulatoren moeten diverse problemen overwonnen worden. De configuratie en het ontwerp van de robots leidt tot hoge orde, complexe, dynamische modellen en specifieke problemen zoals wrijving en flexibiliteit in scharnieren en verbindingspunten. Veel van deze problemen komen in dit proefschrift aan de orde. De oplossingen waarmee de voorgestelde synchronisatie schema's in de experimentele opstellingen konden worden geïmplementeerd worden uitgebreid besproken in dit proefschrift.

Mogelijke uitbreidingen van de voorgestelde synchronisatie ideeën en aanbevelingen voor verder onderzoek naar geregelde synchronisatie worden voorgesteld. De meeste aanbevolen onderzoekslijnen vertegenwoordigen uitdagende onopgeloste problemen. 


\section{Resumen}

Esta tesis estudia la posibilidad de inducir sincronización en sistemas mecánicos. Sincronización de sistemas mecánicos es importante ya que da lugar a sistemas mas robustos y capaces de realizar tareas mas complejas. Por medio de la sincronización varias relaciones e interacciones entre los sistemas son forzadas, tal que algunos objetivos pueden ser satisfechos, por ejemplo minimizar el tiempo de ejecución de la tarea, minimizar el error relativo entre los sistemas, ya sea de posición, velocidad, o ambos. Como resultado se obtienen sistemas mas eficaces y con mejor desempeño. Existe un sin numero de ejemplos de sistemas mecánicos que trabajan bajo un esquema de sincronización, teleoperación de sistemas robóticos maestro-esclavo los cuales son usados en telecirugía o en ambientes peligrosos, maquinaria basada en vibración y que es usada en minería, sistemas multi-robot tales como manos multi-dedo, etc.

El objetivo de esta tesis puede ser formulado como diseñar esquemas de control que aseguren sincronización de dos o mas sistemas mecánicos, los cuales pueden ser idénticos o diferentes. La hipótesis que restringe el diseño del esquema de control para synchronización es que solamente están disponibles mediciones de las posiciones de los sistemas. La ausencia de mediciones de velocidad y aceleración en los sistemas a ser sincronizados es resuelta por medio de observadores de estado basados en modelo. Los valores de velocidad y aceleración que son obtenidos por los observadores son usados en esquemas de control basados en retroalimentación, los cuales aseguran la sincronización de los sistemas mecánicos.

En esta tesis se le da especial atención a sistemas de robot manipuladores, para los cuales dos tipos de sincronización son estudiados: sincronización mutua (cooperación) y sincronización externa (coordinación). No obstante la tesis se enfoca en robot manipuladores, las ideas que se exponen pueden ser aplicadas a sistemas mas generales como por ejemplo: robots móviles, sistemas de varios satélites, sistemas de comunicación, y sistemas electromecánicos tales como generadores eléctricos y motores. En particular la tesis presenta extensiones de las ideas propuestas al caso de seguimiento en robots móviles y formaciones de satélites. Las extensiones propuestas en la tesis presentan las ideas generales que originan el tipo de sincronización deseado en los sistemas mencionados. Las extensiones propuestas son validadas por medio de simulaciones.

Los resultados presentados en esta tesis están avalados por pruebas analíticas y resultados experimentales que muestran la sincronización predicha analíticamente. Esto además de validar los resultados de la tesis da idea del aspecto practico y de aplicación de los esquemas de control para sincronización propuestos en la tesis. La plataforma 
para experimentos esta formada por dos robots industriales instalados en el laboratorio de Tecnología, Dinámica y Control del Departamento de Ingeniería Mecánica de la Universidad Tecnológica de Eindhoven (Technische Universiteit Eindhoven).

En el caso particular de sincronización de robot manipuladores existen varios problemas, aparte del de sincronización, que tienen que ser resueltos para poder implementar un esquema de control. La estructura y diseño de los robots que se desean sincronizar, y que son dos aspectos intrínsecos a cualquier robot, dan lugar a problemas tales como fricción en las articulaciones y motores, flexibilidad en las uniones, así como modelos dinámicos altamente complejos y de orden alto. Varios de los problemas previamente mencionados son revisados en esta tesis y la manera en que fueron resueltos se expone a lo largo de la tesis.

La tesis presenta posibles extensiones de los esquemas de control para sincronización propuestos, así como sugerencias para posibles líneas de investigación en sincronización de sistemas. Las líneas de investigación aquí propuestas representan problemas sin resolver en sincronización de sistemas y la mayoría de ellos surgieron durante la realización de esta tesis. 


\title{
Curriculum vitae
}

\begin{abstract}
Alejandro Rodriguez Angeles was born on the 8th of March 1973 in Tepeji del Rio, a small town near Mexico City. Upon completion of his high school in 1990 he started his academic education in Electronics and Communication Engineering at the National Polytechnical Institute (IPN), Mexico City, Mexico. In 1995 he started his master program at the Department of Electrical Engineering at the Center for Research and Advanced Studies (CINVESTAV), Mexico City, Mexico. As result of two years in the master program he wrote the thesis entitled "Control (Cooperation) of Multi-Robot Manipulators". During the master project he became interested in control theory and robotics. With this motivation he became in 1998 a Ph.D. student at the University of Twente, Enschede, The Netherlands, under the supervision of prof. dr. Henk Nijmeijer. The Ph.D. project was entitled "Synchronization of Mechanical Systems". After almost two years at the University of Twente the Ph.D. project was moved to Eindhoven at the Eindhoven University of Technology. This change of universities gave him the opportunity of experimenting his ideas about synchronization (theoretical so far) in real robots. The results of the Ph.D project are presented in this thesis.
\end{abstract}

